MODELING OF POPULATION DISTRIBUTION IN SPACE AND TIME TO SUPPORT DISASTER RISK MANAGEMENT 



\title{
MODELING OF POPULATION DISTRIBUTION IN SPACE AND TIME TO SUPPORT DISASTER RISK MANAGEMENT
}

\author{
DISSERTATION
}

to obtain

the degree of doctor at the University of Twente, on the authority of the rector magnificus, prof.dr. T.T.M. Palstra,

on account of the decision of the Doctorate Board to be publicly defended

on Friday 8 May 2020 at 16.45 hours

by

Sérgio Manuel Carneiro Freire born on the 14th of May 1972

in Porto, Portugal 
This dissertation has been approved by

Prof. dr.ir. R.V. Sliuzas, supervisor

Prof. dr.ir. M.F.A.M. van Maarseveen, supervisor

ITC dissertation number 382

ITC, P.O. Box 217, 7500 AE Enschede, The Netherlands

ISBN 978-90-365- 4999-8

DOI $10.3990 / 1.9789036549998$

Cover designed by Job Duim Printed by ITC Printing Department

Copyright (c) 2020 by Sérgio Manuel Carneiro Freire, The Netherlands. All rights reserved. No parts of this thesis may be reproduced, stored in a retrieval system or transmitted in any form or by any means without permission of the author. Alle rechten voorbehouden. Niets uit deze uitgave mag worden vermenigvuldigd, in enige vorm of op enige wijze, zonder voorafgaande schriftelijke toestemming van de auteur.

\section{UNIVERSITY OF TWENTE.

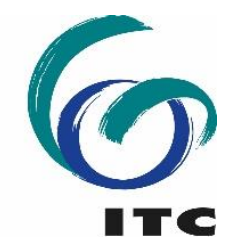


Graduation committee:

\section{Chairman/Secretary}

Prof.dr. F.D. van der Meer

\section{Supervisor(s)}

Prof.dr.ir. R.V. Sliuzas

Prof.dr.ir. M.F.A.M. van Maarseveen

\section{Members}

Prof.dr.ir. R.V. Sliuzas

Prof.dr.ir. M.F.A.M. van Maarseveen

Prof.dr. K. Pfeffer

Prof.dr.ir. A. Stein

dr. C.J. van Westen

Prof. dr. C. Linard

Prof. dr. D. Martin 
Dedicated to Sara and Marta:

"May you build a ladder to the stars And climb on every rung

May you stay forever young"

Bob Dylan 


\section{Foreword}

It became a sort of tradition to equate a PhD to a 'long and arduous journey' - but it certainly was in this case. The work comprising this thesis spans 9 years, two countries and two research institutions where I have lived and worked in this period: the FCSH-UNL (Lisbon, Portugal) and the EC-JRC (Ispra, Italy).

My interest in modeling population distribution was born in the previous millennium, during my time as graduate student at the University of Kansas (USA). There on the edge of the Great Plains I had the fortune of being exposed to the first version of LandScan global population grid (1998) and meeting its visionary author, Jerry Dobson. I was struck by the apparent simplicity and broad reach of the concept that characterize bright developments, and was hooked on the possibilities.

After leaving KU I continued to develop my population models, and around 2008 I also directed my interest to their applications, especially in Disaster Risk Management, as that seemed to me the domain where most societal benefits were to be gained. I did this mostly on my own time while working on other research projects and activities (e.g. teaching GIS), as I had no funding for this specific line of research. I tried as much as possible to connect topics and during those very productive years managed to develop several applications of improved population distribution across different domains. Having amassed some research experience and publications, around 2012 I started inquiring about the possibility of turning some of this body of work and publications into a PhD thesis, but this was deemed not viable. I moved on, embracing a new challenge at the EC-JRC in Italy.

In 2015, with more than 100 scientific publications under my belt (and having contributed to two PhDs) an opportunity appeared that could lead to earning that degree that is a necessary (but not sufficient) step for a researcher to improve his job prospects. Through Richard Sliuzas, whom I had met earlier, I applied to the PhD program at the ITC Faculty, University of Twente (a perfect fit for my topic), submitted a proposal, and formally enrolled in 2016. At this point I assumed it would be rather straightforward to build a decent PhD thesis as an external student by combining my ongoing research at JRC with that already published.

I was wrong, of course - being with small children in a foreign country, having a wife with frequent business travels, and an absorbing job, this was in hindsight not a bright idea, and one that I regretted often. Paradoxically, a truly smart person would have chosen otherwise than going for a PhD. This diverted much energy, time, and patience from important activities and persons, and represented a high cost for an unknown benefit. And yet here I 
am, nearing completion. In the process, I confirmed that earning a PhD is as much a proof of research skills as it is a trial of endurance, resilience, and stamina (the fact that many fail this test, enduring mental or physical damage, would warrant more attention from those involved in awarding these degrees).

As I write this in Lombardy, we are in the middle of the COVID-19 pandemic, which started just when I was in the home stretch of finalizing this thesis. Besides prompting a worldwide lockdown and crisis without an end in sight (and postponing my well-deserved vacation), this epidemic tragically underlines the importance of disaster risk management and how much work remains to improve it. 


\section{Acknowledgements}

Most of the publications comprising the core of this thesis have resulted from international collaborations, and I am especially pleased and proud of this circumstance. So I start by thanking all 12 co-authors, from seven nationalities and four institutions in two continents - I was honored to have you on board. Special thanks to my buddy and co-author extraordinaire Chris Aubrecht; my initial solitary efforts at population modeling and applications had a boost when we met at Gi4DM 2009 in Prague and joined interests and forces for a fruitful collaboration and friendship that I deeply cherish. I am also appreciative of the many people with whom I crossed paths and discussed ideas at numerous scientific meetings (in four continents), many of whom I came to befriend.

Although this thesis features 'only' five publications as core chapters, in two of my lines of research merging here I have authored and co-authored $50+$ scientific publications, of which 45 are cited in this thesis. Therefore I also want to acknowledge that large group of co-authors, with whom I have learned so much.

I want to thank the people and institutions who contributed data to the work supporting this thesis, especially important when this research was unfunded: António Costa, Francisco Costa, Albertina Lérias (Geopoint Lda.), José L. Zêzere (RISKam group/CEG-IGOT), Nuno Gomes, and Lisbon Metropolitan Area.

At FCSH-UNL in Lisbon I thank my colleagues from e-GEO and especially José A. Tenedório and Carlos Pereira da Silva for supporting me in this line of research when no one else was.

I thank my teammates at JRC, in particular Martino Pesaresi for the encouragement and the enlightening discussions, but also my good comrades of office 112: Aneta Florczyk, Marcello Schiavina, and Michele Melchiorri Michele, for assistance with the template and formatting, grazie mille.

I am grateful to ITC and my supervisors, Prof. Martin van Maarseveen and Prof. Richard Sliuzas for accepting me as their student and for their supervision. Richard, thanks so much for your guidance and generosity and apologies for my impatience. I also express my gratitude to the distinguished PhD Committee Members, in particular to the external members (Prof. Martin and Prof. Linard) for accepting to serve on this jury. I extend my appreciation to Petra Weber and ITC staff for all assistance.

To my former colleagues and life friends Hugo Carrão and António Nunes um grande abraço for their ever-present encouragement. A special thank you is due to Alex de Sherbinin for showing me that this could be done and that it was not too late to do it. 
Last but certainly not least, I thank all my family and especially my wife Ana for her support and for being so resilient during these last couple of tough years for both of us. I owe a special recognition and apology to my daughters Sara and Marta, who (while babies) kept me company during long nights while I did much of the early work supporting this thesis. Then more recently, this thesis was put together stealing also their time - and therefore it is dedicated to them. 


\section{Table of Contents}

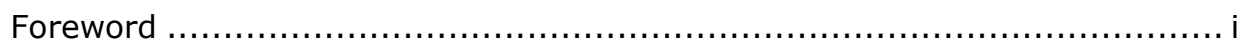

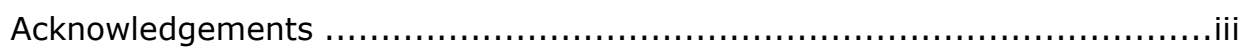

List of Figures.......................................................................... vii

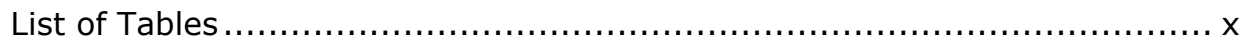

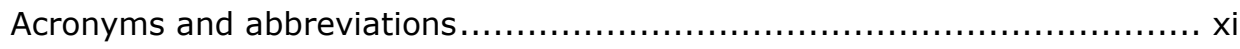

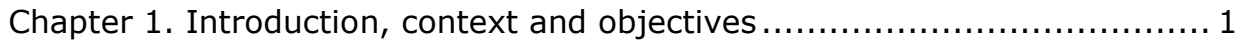

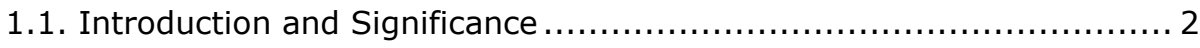

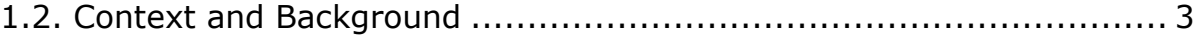

1.2.1 Population exposure and risk landscape, governance, and trends . 3

1.2.2 Mapping exposure for assessing populations at risk .................. 7

1.2.3 Beyond exposure: the relevance of population in integrated DRM 13

1.2.4 Background: Modeling and mapping of population distribution in space and time (for exposure analysis and DRM) ........................ 17

1.3 Objectives and structure of the Dissertation.............................. 27 Chapter 2. Modeling of spatio-temporal distribution of urban population at high-resolution - value for risk assessment and emergency management .. 37

2.1 Introduction................................................................. 38

2.1.1 Population distribution and emergency management.............. 38

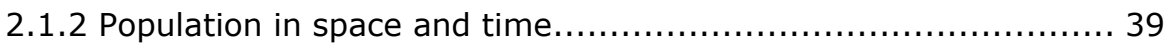

2.2 Methods ..................................................................... 40

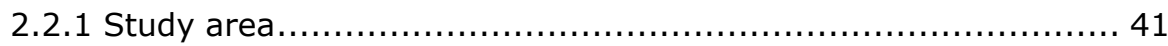

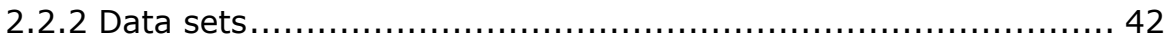

2.2 .3 Model.................................................................. 43

2.3 Results........................................................................ 45

2.3.1 Verification and validation ........................................ 45

2.4 Sample applications ....................................................... 46 2.4.1 Case study $A$ : technological hazard (airborne toxic plume release) 46

2.4.2 Case study B: natural disaster (earthquake) ........................ 47

2.4.3 Case study C: terrorist attack (bombing of shopping center) ...... 48

2.4.4 Case study $\mathrm{D}$ : planning of best route for hazardous materials

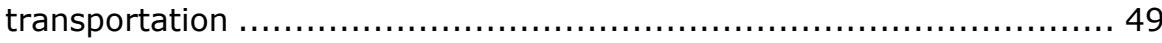

2.5 Conclusions .............................................................. 51

Chapter 3. Integrating population dynamics into mapping human exposure to

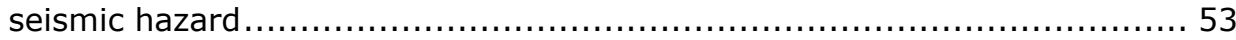

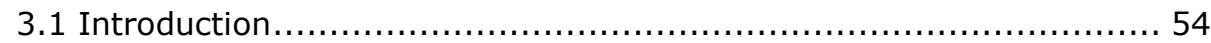

3.1.1 The importance of population dynamics for disaster risk assessment

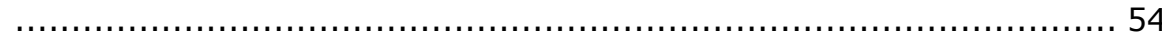

3.1.2 Population exposure to seismic hazard ............................ 56 


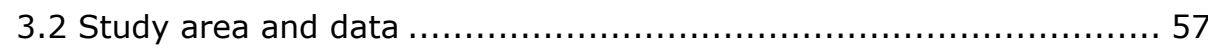

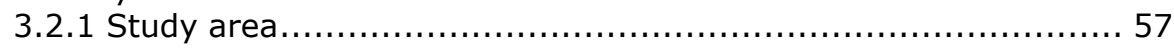

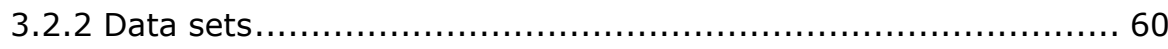

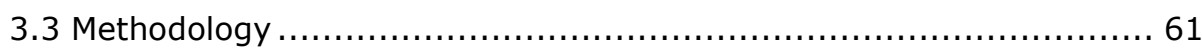

3.3.1 Modeling spatio-temporal population distribution ................... 61

3.3.2 Assessing population and classifying human exposure to seismic hazard 63

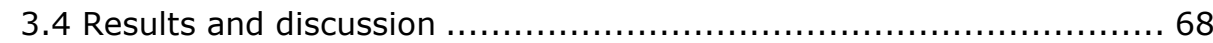

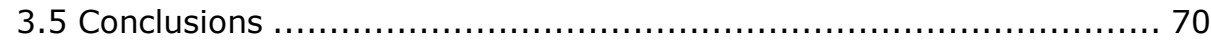
Chapter 4. Advancing tsunami risk assessment by improving spatio-temporal population exposure and evacuation modeling ............................... 73

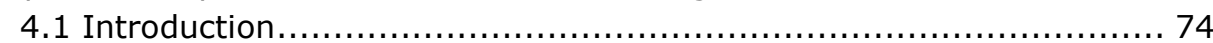

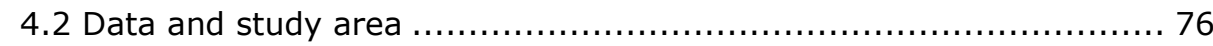

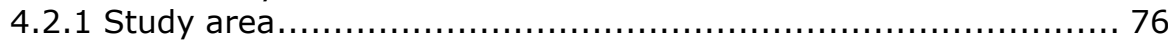

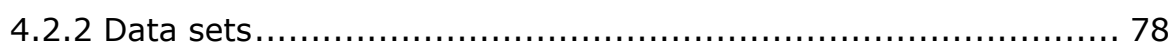

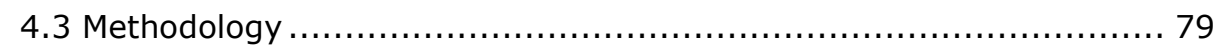

4.3.1 Modeling nighttime and daytime population distribution ........... 79

4.3.2 Assessing population exposure to tsunami ...................... 81

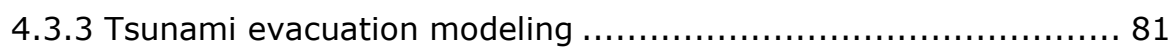

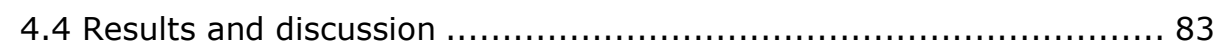

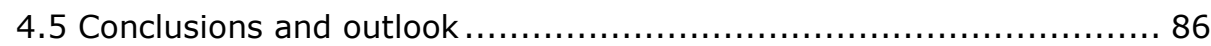

Chapter 5. Enhanced data and methods for improving open and free global population grids: putting 'leaving no one behind' into practice ............... 89

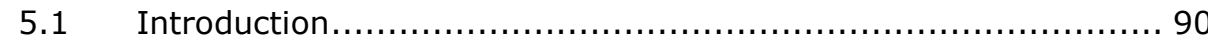

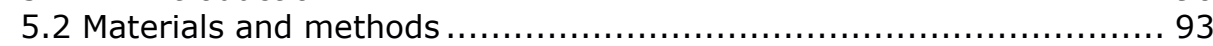

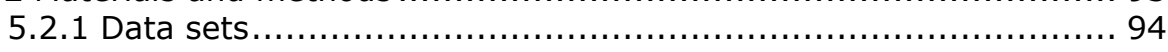

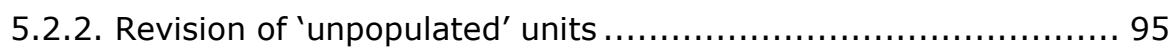

5.2.3 Harmonization of population and settlement data along coastlines

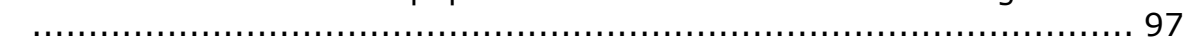

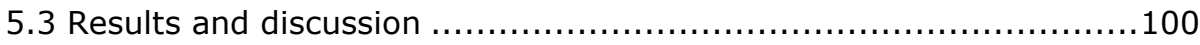

5.3.1 Revision of unpopulated units ........................................ 100

5.3.2 Harmonization of population and settlement data along coastlines

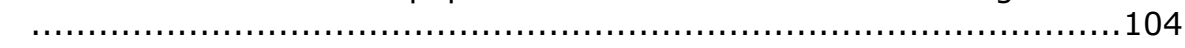

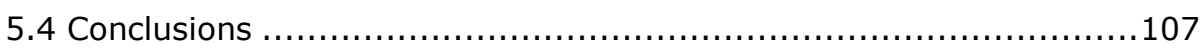
Chapter 6. An Improved Global Analysis of Population Distribution in Proximity to Active Volcanoes ...................................................... 109

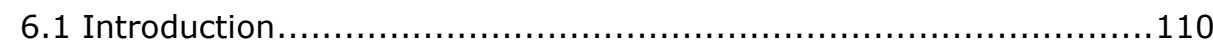

6.1.1 Potential Global Population Exposure to Volcanic Hazard -Narrowing Data Gaps............................................................ 112

6.1.2 The Contribution of the Global Human Settlement Layer (GHSL) to

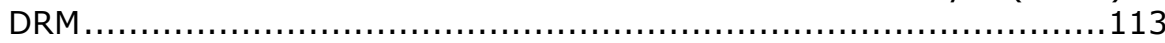

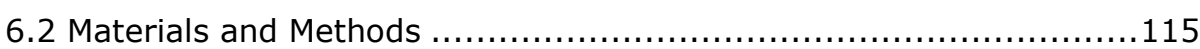




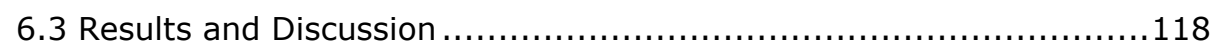
6.3.1 Global Population Distribution from 1975 to 2015 in Relation to Volcanism

6.3.2 Population Distribution from 1975 to 2015 in Relation to Volcanism, in Southeast Asia and Central America .................................124

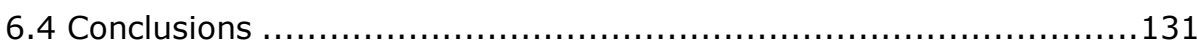

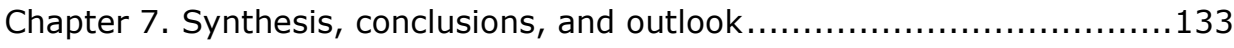

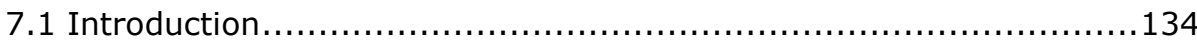

7.2 Main results: Modeling population distribution in space and time to support Disaster Risk Management ....................................... 134

7.2.1 Chapter 2: Modeling of spatio-temporal distribution of urban population at high-resolution - value for risk assessment and emergency management.

7.2.2 Chapter 3: Integrating population dynamics into mapping human exposure to seismic hazard..... .136

7.2.3 Chapter 4: Advancing tsunami risk assessment by improving spatiotemporal population exposure and evacuation modeling 138

7.2.4 Chapter 5: Enhanced data and methods for improving open and free global population grids: putting 'leaving no one behind' into practice..140

7.2.5 Chapter 6: An Improved Global Analysis of Population Distribution in Proximity to Active Volcanoes, 1975-2015

7.3 Other research contributions: cross-disciplinary benefits and applications of the produced population distribution data for modelling, assessing impacts, and measuring access to services and resources. ..................144

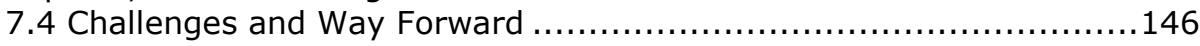

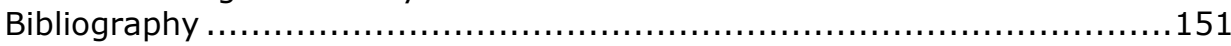

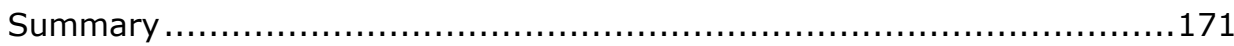

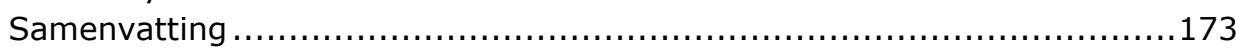




\section{List of Figures}

Figure 1 A typology of hazards and respective characteristics. Source: UNEP/GRID Arendal (http://maps.grida.no/go/graphic/typology_of_hazards).

Figure 2 DRM process and phases, conceptualized as cycle and spiral. Source.

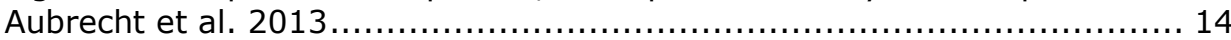
Figure 3 Location of the study area in Portugal and in the Lisbon Metro Area (LMA) (Source: CAOP) . .................................................... 42 Figure 4 Flowchart of main tasks involved in the model: input data are noted in light gray, secondary products in orange, and main results noted in bold and

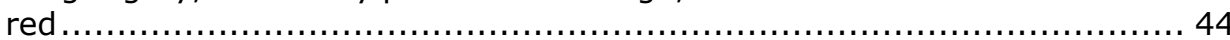
Figure 5 Case study A: airborne toxic plume release in Oeiras (Source: IGP;

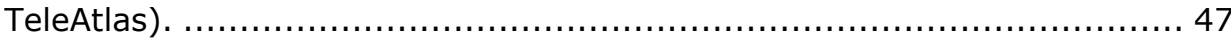
Figure 6 Case study B: earthquake affecting downtown Cascais (Source: IGP;

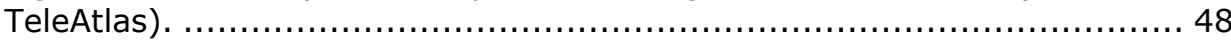
Figure 7 Case study C: terrorist attack in a Cascais shopping center (Source: IGP; TeleAtlas). .............................................................. 49 Figure 8 Case study $\mathrm{D}$ : best route considering the population distribution (Source: IGP; TeleAtlas).................................................. 50 Figure 9 Study area - Lisbon Metropolitan Area (LMA) ....................... 58 Figure 10 Seismic Intensity map for the study area (background from Google

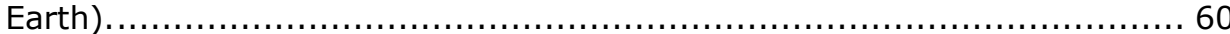
Figure 11 Nighttime population density and seismic zones (background from

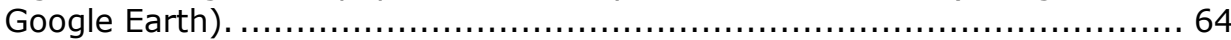
Figure 12 Daytime population density and seismic zones (background from

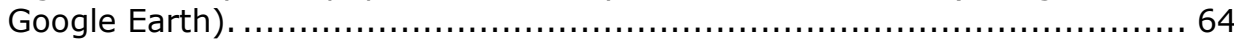
Figure 13 Classification approach to categorize human exposure levels ...... 65 Figure 14 Map of nighttime human exposure to seismic hazard ..............6 67 Figure 15 Map of daytime human exposure to seismic hazard ..................67 67 Figure 16 Study area - Lisbon Metropolitan Area (LMA) .................... 77 Figure 17 Tsunami Inundation Susceptibility map for the LMA (background

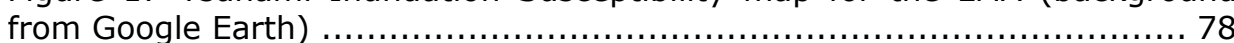
Figure 18 Nighttime (A) and daytime (B) population density and Tsunami Inundation Susceptibility zones (background from Google Earth) ............. 81 Figure 19 Study area for Tsunami evacuation modeling $\ldots \ldots \ldots \ldots \ldots \ldots \ldots \ldots \ldots 2$ Figure 20 Nighttime (A) and daytime (B) Tsunami evacuation modeling..... 85 Figure 21 Flow chart of the 'split and merge' approach ....................... 97 Figure 22 Examples of detected discrepancies (patches larger than $1 \mathrm{~km}^{2}$ ) between GPWV4 (blue line) and GHS-BUILT 2014 (orange) along the coasts of (A) Japan (JPN), (B) Tunisia (TUN), (C) China (CHN), and (D) Romania (ROU) .............................................................................. 99

Figure 23 Global distribution of census units without population in GPWv4.10 data and those flagged based on the automated procedure developed (USA not considered). Note that existence of unpopulated units is merely a feature of census design and not of the landscape .................................... 100 Figure 24 Examples of application of the 'split and merge' approach to census units incorrectly deemed as unpopulated in (A) Guyana and (B) Lebanon, before (1) and after (2) the procedure is applied. Orange filling and red dashed boundary represent the original problematic polygon; blue solid line delineates 
borders of upper administrative level; grey solid polygons are correctly declared unpopulated polygons; solid coloured areas are the resulting polygons; areas outside the processed administrative unit are shaded......102 Figure 25 Illustration of resulting $250-\mathrm{m}$ population grids for 2015 in (A) Guyana and $(B)$ Lebanon ............................................... 104 Figure 26 Visually confirmed coastline discrepancy patches larger than $1 \mathrm{~km}^{2}$. .105

Figure 27 Example illustrating situation (A) before and (B) after manual harmonization of a stretch of Caspian Sea coastline in Russia. Solid black lines enclose original census units; black pixels denote mapped built-up areas (shaded: already within census boundaries; solid: outside original census

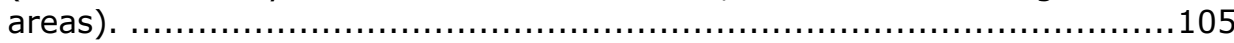
Figure 28 Example of automated approach to mitigate inconsistencies along the coastline of Ukraine. This example shows the generation of a 2-km buffer that was split using the 'split and merge' approach. Solid black lines enclose original census units; solid blue line represents the extended boundary into the sea through 2-km buffer; solid red lines represent the splitting of buffer into parts assigned to the nearest neighbouring unit; black pixels represent mapped built-up areas (shaded: already within census boundaries; solid: outside original census areas). 106

Figure 29 Distribution of the two global datasets of volcanoes used in this study: Holocene Volcano List v4.7.6 (HV) and Significant Volcanic Eruption Database (SV) (World Mollweide projection). 117

Figure 30 Cumulative population as a function of radial distance to Holocene

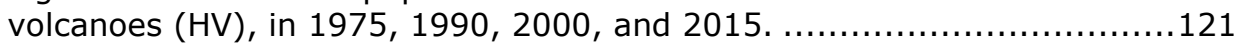
Figure 31 Cumulative population as a function of radial distance to volcanoes in Significant Volcanic Eruption Database (SV), in 1975, 1990, 2000, and 2015. ..121

Figure 32 Average population density as a function of radial distance to Holocene volcanoes (HV), in 1975, 1990, 2000, and 2015.................122 Figure 33 Average population density as a function of radial distance to volcanoes in Significant Volcanic Eruption Database (SV), in 1975, 1990, 2000, and 2015.

Figure 34 Selected distances from Holocene volcanoes in (A) Southeast Asia and (B) Central America. .125

Figure 35 Average population density in Southeast Asia (SEAsia) and Central America (CAm) as a function of radial distance of Holocene Volcanoes (HV), in 1975 and 2015. 129

Figure 36 Average population density in Southeast Asia (SEAsia) and Central America (CAm) as a function of radial distance of volcanoes in Significant Volcanic Eruption Database (SV), in 1975 and 2015. 129 Figure 37 Island of Java (Indonesia), showing selected distances from Holocene volcanoes, main cities, and population distribution in 2015 from GHS-POP (World Mollweide projection). 


\section{List of Tables}

Table 1 Usefulness of geospatial demographic data (with focus on total population counts and density) in DRM phases. Source: author own elaboration

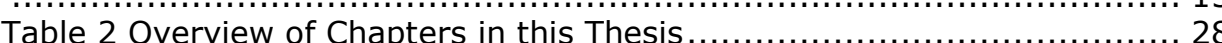
Table 3 Main characteristics of chapters in this thesis in relation to main research topics and concepts; main innovative aspects highlighted in bold . 34 Table 4 (cont.) Main characteristics of chapters in this thesis in relation to main research topics and concepts; main innovative aspects highlighted in bold. 35 Table 5 Main input datasets used for modeling nighttime and daytime

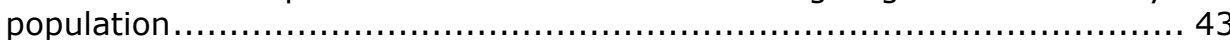

Table 6 Total exposed population in each case study $\ldots \ldots \ldots \ldots \ldots \ldots \ldots \ldots \ldots . \ldots \ldots$

Table 7 Total exposed population along each route and period .................. 50

Table 8 Nighttime and daytime population in the municipalities of the Lisbon Metropolitan Area, in 2001 (derived from INE, 2001, 2003) .................. 59

Table 9 Main input data sets used for modeling population distribution ...... 61 Table 10 Population exposed to seismic intensity levels in nighttime and

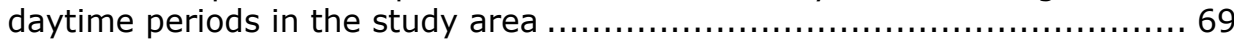
Table 11 Total surface and population in each human exposure class in nighttime and daytime periods in the study area .............................. 69 Table 12 Main input data sets used for modeling population distribution .... 79 Table 13 Population exposed to Tsunami inundation levels in nighttime and

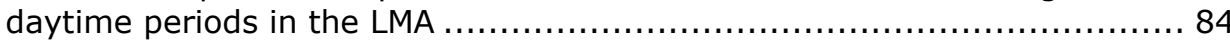
Table 14 Population remaining in hazard zone and successful evacuees after different time intervals of tsunami evacuation ............................... 85 Table 15 List of countries in which problematic polygons were selected.....101 Table 16 Population (in millions and as percentage of World total) by radial distance from Holocene volcanoes (HV) in 1975, 1990, 2000, and 2015. ..119 Table 17 Compound annual growth rate of population by radial distance from Holocene volcanoes (HV) for each time interval. ..............................119 Table 18 Population (in millions and percentage of World total) by radial distance from volcanoes with significant eruptions (SV) in 1975, 1990, 2000, and 2015.

Table 19 Compound annual growth rate of population by radial distance of volcanoes with significant eruptions (SV) for each time interval. ..............120 Table 20 Population (in millions and percentage of region total) by radial distance of Holocene volcanoes (HV) and volcanoes in Significant Volcanic Eruption Database (SV) in 1975, 1990, 2000, 2015, in Southeast Asia and Central America. 125

Table 21 Compound annual growth rate of population by radial distance of Holocene volcanoes (HV) and volcanoes in the Significant Volcanic Eruption Database (SV), for each time interval, in Southeast Asia and Central America. 


\section{Acronyms and abbreviations}

\begin{tabular}{|c|c|}
\hline $2-\mathrm{D}$ & Two dimensions \\
\hline $3-D$ & Three dimensions \\
\hline ANPC & Autoridade Nacional de Protecção Civil \\
\hline AOI & Area Of Interest \\
\hline BU & Built-up \\
\hline CAPRA & Central American Probabilistic Risk Assessment \\
\hline CIESIN & Center for International Earth Science Information Network \\
\hline CLC2000 & Corine Land Cover 2000 \\
\hline CNN & Convolutional Neural Networks \\
\hline COP21 & Conference of the Parties 21 \\
\hline COS90 & Carta de Uso e Ocupação do Solo \\
\hline CUT & Coalition for Urban Transitions \\
\hline DRC & Democratic Republic of the Congo \\
\hline DRM & Disaster Risk Management \\
\hline DRR & Disaster Risk Reduction \\
\hline EC & European Commission \\
\hline EEA & European Environment Agency \\
\hline EFAS & European Flood Awareness Systems \\
\hline EM & Emergency Management \\
\hline EM-DAT & Emergency Events Database \\
\hline EMS & Emergency Management Service \\
\hline ENACT & ENhancing ACTivity and Population Mapping \\
\hline ESM & European Settlement Map \\
\hline EU & European Union \\
\hline FEMA & Federal Emergency Management Agency \\
\hline FUA & Functional Urban Area \\
\hline GADM & Global Administrative Areas \\
\hline GAR & Global Assessment Report on Disaster Risk Reduction \\
\hline GDACS & Global Disaster Alert and Coordination System \\
\hline GDP & Gross Domestic Product \\
\hline GED & Global Exposure Database \\
\hline GED4GEM & Global Exposure Database for the Global Earthquake Model \\
\hline GEG & Global Exposure Database for GAR 2013 \\
\hline GFDRR & Global Facility for Disaster Reduction and Recovery \\
\hline GHSL & Global Human Settlement Layer \\
\hline GIS & Geographic Information System \\
\hline GITEWS & German-Indonesian Tsunami Early Warning System \\
\hline GloFAS & Global Flood Awareness Systems \\
\hline GPW & Gridded Population of the World \\
\hline GRUMP & Global Rural-Urban Mapping Project \\
\hline GUF & Global Urban Footprint \\
\hline GVP & Global Volcanism Program \\
\hline HOT & Humanitarian OpenStreetMap \\
\hline HRSL & High Resolution Settlement Layer \\
\hline HV & Holocene Volcano List \\
\hline HYDE & History Database of the Global Environment \\
\hline INE & Instituto Nacional de Estatística \\
\hline INFORM & Index for Risk Management \\
\hline IOC & Intergovernmental Oceanographic Commission \\
\hline
\end{tabular}




$\begin{array}{ll}\text { IPCC } & \text { Intergovernmental Panel on Climate Change } \\ \text { IPCC } & \text { Intergovernmental Panel on Climate Change } \\ \text { ISO } & \text { International Organization for Standardization } \\ \text { LBSM } & \text { Location-Based Social Media } \\ \text { LiDAR } & \text { Light Detection and Ranging } \\ \text { LMA } & \text { Lisbon Metropolitan Area } \\ \text { LULC } & \text { Land Use/Land Cover } \\ \text { MAUP } & \text { Modifiable areal unit problem } \\ \text { MRI } & \text { Mortality Risk Index } \\ \text { NATECH } & \text { Natural and technological hazards } \\ \text { NGO } & \text { Non-Governmental Organization } \\ \text { NOAA } & \text { National Oceanic and Atmospheric Administration } \\ \text { NRC } & \text { National Research Council } \\ \text { NSO } & \text { National Statistical Office } \\ \text { NSTC } & \text { National Science and Technology Council } \\ \text { PAGER } & \text { Prompt Assessment of Global Earthquakes for Response } \\ \text { PAGER } & \text { Prompt Assessment of Global Earthquakes for Response } \\ \text { PEI } & \text { Population Exposure Index } \\ \text { POI } & \text { Point of Interest } \\ \text { PROTAML } & \text { Regional Plan for Territorial Management for the Lisbon } \\ & \text { Metropolitan Area } \\ \text { SDG } & \text { Sustainable Development Goals } \\ \text { SV } & \text { Significant Volcanic Eruption Database } \\ \text { UCDB } & \text { Urban Centre Database } \\ \text { UN } & \text { United Nations } \\ \text { UNDESA } & \text { United Nations Department of Economic and Social Affairs } \\ \text { UNDP } & \text { United Nations Development Programme } \\ \text { UNDRR } & \text { United Nations Office for Disaster Risk Reduction } \\ \text { UNECOSOC } & \text { United Nations Economic and Social Council } \\ \text { UNISDR } & \text { United Nations International Strategy for Disaster Reduction } \\ \text { USGS } & \text { United States Geological Survey } \\ \text { VEI } & \text { Volcanic Explosivity Index } \\ \text { VGI } & \text { Volunteered Geographic Information } \\ \text { VPI } & \text { Volcano Population Index } \\ \text { VRC } & \text { Volcanic Risk Coefficient } \\ & \end{array}$


Chapter 1. Introduction, context and objectives 


\subsection{Introduction and Significance}

Geospatial information on population distribution and densities is one of the most fundamental and critical datasets to study human presence and settlement, interactions, impacts, and vulnerabilities (Leyk et al. 2019), in applications spanning the domains of research, decision and policy-making. For example, in the context of policy support, the post-2015 international development agreements (i.e. Sendai, Sustainable Development Goals-SDGs, Paris Agreement/COP21, UN New Urban Agenda) place great demands and responsibility on geospatial data, and in particular on that related to population. As human life is the most important value to protect from disasters, assessing population exposure to actual or potential disasters is key to Disaster Risk Management (DRM) and Reduction measures (DRR). Consequently, suitable population distribution data can benefit all phases of the disaster management cycle, e.g. baseline risk analysis and impact assessment, mitigation, preparedness (including early warning and evacuation), response and rehabilitation (Freire 2010).

Risk results from the intersection, in space and time, of hazard, exposure, and vulnerability. However, human exposure is dynamic and has been increasing in potential magnitude and complexity due to population growth and the expansion of human activities to hazardous areas, as a result of strong clustering of people in settlements (e.g. from megacities to small villages), due to increasing population mobility and dynamics (especially high frequency: for work and study, but also due to tourism, migration, and displacement). Despite its importance for risk management, mapping of human vulnerability and population exposure has traditionally lagged behind hazard modeling efforts (Pelling 2004), in terms of accuracy, detail, and currency (Smith et al. 2019).

Simultaneously, the risk and disaster landscape is becoming ever more complex and interconnected (UNDRR 2019), and anthropogenic actions are changing the very concept of 'natural' disaster (Peduzzi 2019). Against this evolving background, assessing potential or actual human population exposure requires geo-information on population distribution at a range of spatial and temporal scales, as disasters can occur at any time, and their spatial effects may range from local to global scales.

There are significant challenges and trade-offs affecting spatio-temporal population modeling. For effective support to DRM, geospatial population data should be reliable, up-to-date, have sufficient resolution (spatial, temporal, thematic), and be readily available (i.e. either produced beforehand or be rapidly computable on-demand). Such population data are still lacking for many countries and regions, both rich and poor, and conducting DRM at global scale would certainly benefit from complete, consistent, and integrated population exposure datasets. 
If well-developed, such population datasets are multi-purpose and yield crossdisciplinary benefits in a range of application domains: these include spatial planning (urban, regional, infrastructure, public facilities), environmental assessment, health and epidemiology, and GeoMarketing.

This thesis comprises contributions of population distribution modeling to advancing Disaster Risk Management and Reduction efforts by:

(i) developing geospatial models that improve population distribution datasets at a range of relevant spatial and temporal scales and resolutions;

(ii) applying those data to (real) disaster risk scenarios by combining geospatial population layers with geophysical hazard maps;

(iii) using spatial analysis for quantitatively and qualitatively assessing human exposure to specific hazards and levels and for cartographic representations and visualization, and

(iv) discussing the findings, their impacts as well as contributions to DRM.

While the focus is on the spatio-temporal dynamics of human exposure, the research considers related aspects such as population definition, geospatial data and technology, spatio-temporal scales, hazard types and their characteristics, and the specific population related information requirements throughout the Disaster Risk Management Cycle.

In this initial chapter we present the main concepts involved in this thesis, discuss the relevance and implications of mapping and assessing human exposure to hazards and disasters, and list efforts, challenges, and contributions of the geospatial modeling population distribution for Disaster Risk Management (DRM). Additionally, we provide some context and background on spatio-temporal population modeling.

\subsection{Context and Background}

\subsubsection{Population exposure and risk landscape, governance, and trends}

Disasters, either resulting from natural or man-made hazards (technological accidents, terrorism) frequently occur with little or no warning and can potentially affect people at local, continental, or even at global scales (Gill and Malamud 2014). According to EM-DAT (EM-DAT 2018), globally between the years 2000 and 2017 an average of at least 193 million people per year were affected by disasters ${ }^{1}$.

1 This figure is likely underestimated owing to the combination of impact threshold adopted in definition for disaster and the fact that it includes only climate-related and geophysical events. 
Geospatial information on population distribution (e.g. mapping inhabited vs uninhabited areas) and densities (i.e. people $/ \mathrm{km}^{2}$ ) is one of the most fundamental and critical datasets to study human presence and settlement, interactions, impacts, and vulnerabilities (Leyk et al. 2019). Various post-2015 international development agreements (i.e. Sendai, SDGs, Paris Agreement/COP21, UN New Urban Agenda) place great demands and responsibility on geospatial data, and in particular on that related to population. Of the seven global targets in the Sendai Framework for Disaster Risk Reduction 2015-2030 (UNISDR 2015a), three explicitly focus on population and require population data for their monitoring and assessment:

Target (a) Substantially reduce global disaster mortality by 2030, aiming to lower average per 100,000 global mortality rate in the decade 2020-2030 compared to the period 2005-2015.

Target (b) Substantially reduce the number of affected people globally by 2030, aiming to lower average global figure per 100,000 in the decade 2020 2030 compared to the period 2005-2015.

Target $(g)$ Substantially increase the availability of and access to multi-hazard early warning systems and disaster risk information and assessments to the people by 2030.

With the recognition of the interactions and interdependencies between DRR and development (i.e. the "risk-global change-sustainability nexus"2) in all other post-2015 International agendas some elements of DRR are included, and DRR objectives have ties with SDG goals 1.5, 11.5, 11.B, 13.1 (UNISDR 2015a). The implementation of these International Frameworks provides an opportunity to address underlying risk drivers and encourage a deeper understanding of socioeconomic and environmental vulnerability. They also create opportunities to generate and improve data and statistical capacity for monitoring and decision-making, without which the SDGs may fail (Espey 2019). Disaggregated data sets and statistical data, previously scarce in the disaster risk realm, are now prerequisites for measuring risk-informed sustainable development, with improvements in data availability, quality and accessibility being expected (UNDRR 2019).

Disaster risk has several definitions ${ }^{3}$ and there exist various approaches for its assessment and mapping, even in the context of natural hazard research and practice (Adger 2006; Birkmann 2006; Villagrán 2006). Until 2009 the United Nations (UN), for example, defined disaster risk as a function of hazard probability and vulnerability, the latter resulting from a combination of

2 As in Peduzzi 2019: The Disaster Risk, Global Change, and Sustainability Nexus. Sustainability, 11, 957.

3 An inventory was compiled by Thywissen 2006: Components of Risk. A Comparative Glossary, UNU Institute for Environment and Human Security. 
(population) exposure and ability to cope (UNDP 2004). One practical definition currently widely accepted conceptualizes risk as resulting from the product of three main elements: hazard, exposure, and vulnerability (Dao and Peduzzi 2003). The conceptualization of the Risk Triangle proposed by Crichton (1999) sees these elements as connected sides which enclose and determine a risk space or area. These conceptualizations have at least two important implications, namely that (i) risk is null in the absence of one component, and that (ii) wrongly estimating one of these components necessarily affects the accuracy of the overall risk mapping and analysis. While it is generally assumed that all components must be spatially coincident for a risk to exist (e.g. Tomlinson, 2011), this understanding overlooks the importance of time. However the components of risk are also highly sensitive to spatial and temporal variation (Cutter 2003; Aubrecht et al. 2012a; Aubrecht et al. 2013). Therefore and more accurately, one can say there is risk if all components coincide in space and time. While risk is spatial, requiring spatially-explicit data for mapping and assessment, it is also temporal and dynamic in nature, requiring regular re-assessment (Peduzzi 2019).

Exposure ${ }^{4}$ in this context essentially refers to the "people, property, systems, or other elements present in hazard zones that are thereby subject to potential losses" (UNISDR 2009), while population exposure refers more strictly to the human occupancy of hazard zones (Cutter 1996). People are unquestionably the most important element to protect from hazards and threats, and the distribution and density of the overall population is a rather basic geographical indicator. Therefore, in the early 2000 's, a progressive recognition started that accurately estimating population exposure was a fundamental component of catastrophe loss modeling, one element of effective risk analysis and emergency management (Chen et al. 2004; FEMA 2004). Some studies even indicate that exposure data have the greatest influence on loss estimation from risk models (Chen et al. 2004; Lavakare and Mawk 2008).

Results from the Mortality Risk Index (MRI) conducted for GAR 2009 (UNISDR 2009) showed that the intensity of hazard, level of exposure, poverty, and bad governance were the main underlying factors of risk, and that exposure was the main factor in higher intensity hazards (Peduzzi et al. 2009a; 2012). In a convergence of findings by different risk communities, also the 2012 IPCC Special Report on Extreme Events states that "increasing exposure of people and economic assets has been the major cause of long-term increases in economic losses from weather- and climate-related disasters (high confidence)" (IPCC 2012). It is safe to say that generally, while the importance

4 Definition was again recently modified to "the situation of people, infrastructure, housing, production capacities and other tangible human assets located in hazard-prone areas (UNISDR 2017)". 
of vulnerability decreases with hazard intensity, the relevance of exposure increases (Cardona et al. 2012).

Increases in global exposure to natural hazards have largely been driven by population growth and urbanization rates (Huppert and Sparks 2006; Neumann et al. 2015; Bowman et al. 2017). Not only human exposure has been increasing in potential magnitude but also in complexity due to population growth and expansion to hazardous areas, due to the strong clustering of people in settlements (e.g. megacities), which become highly concentrated locations of exposure (Chester 2000, Gu et al. 2015). When large settlements are affected by a disaster, losses can be disproportionately large (e.g. Hurricane Katrina in New Orleans in 2005). Also, increasing population density and mobility has been contributing to growing vulnerability of social systems (EEA, 2010), and growing population dynamics (especially high frequency movements: for work and study, but also due to tourism, migration, and displacement) (Kellens et al. 2012) are putting more people at risk, often unbeknown (Wieland et al. 2012). Population mobility spans the globe. For instance, the 2004 Indian Ocean earthquake and tsunami disaster highlighted how significant numbers of international tourists can be affected by hazards (Satake 2014).

Furthermore, evidence indicates that not only exposure of persons and assets is increasing, but that in both higher and lower income countries it has increased faster than vulnerability has decreased, thus generating new risks (UNISDR 2015a; UNDRR 2019). This is likely to continue, as "an increase in exposure induced by population and economic growth has been identified as the main factor inflating disaster risk in the near future" (Peduzzi 2019). In a recent prospective study of human exposure to dangerous heat in African cities under multiple scenarios, Rohat et al. (2019) concluded that future exposure is predominantly driven by changes in urban population alone or by concurrent changes in climate and urban population.

Despite its importance, efforts to asses and map exposure, have lagged behind. This has its roots in the early perception of risk as being hazard-based, implying that traditionally modeling and analysis of disaster risk has been undertaken by physical scientists whose work is more focused on the physical processes of hazard than on the vulnerability and exposure components, especially the human components (Smith et al. 2019). In the context of risk of river floods, these authors admit "this is concerning, as arguably we know even less about the location of people and assets, and the impact of hazards on them, than we do about the frequency and nature of the flood hazard events" (Smith et al. 2019, p. 2). Also, until not long ago the availability and integration of socioeconomic variables into geospatial risk models implemented within a Geographic Information System (GIS) remained a challenge (EC 2010). Perhaps the smaller amount of attention devoted to the importance, gaps and challenges in modeling exposure is reflected in the very definition of 
risk proposed by the UN, mentioned above (UNDP 2004), where exposure was embedded within vulnerability and did not emerge as an autonomous component of the risk equation. This component has progressively received more attention in last decade, with the realization that exposure modeling has a critical role to play in risk assessment, and that needs and challenges associated with this component are far from being solved (GFDRR 2014). These challenges include need for information on exposure that is up-to-date, detailed, covers wide geographical areas with spatial and temporal consistency, and accounts for dynamics of exposure (Wieland et al. 2012; GFDRR 2014).

Simultaneously, the risk and disaster landscape is becoming ever more complex and interconnected (UNDRR 2019): many 'classical' hazards increase in frequency and intensity, triggering cascading effects, and are joined by new forms of terrorism and 'invisible' threats (e.g. epidemics, but also cyber terrorism), while anthropogenic actions are changing and challenging the very concept of 'natural' disaster (Peduzzi, 2019). The Sendai Framework has widened the view of the world's risks, by considering natural and man-made hazards, stressing the need for action in the four priority areas at local, national, regional and global levels (UNISDR 2015a). Those actions should be supported by more detailed and data-driven understanding of disaster risk, at three geographical scales: global, national, and local. The combination of differences in levels of risk governance, actors, scope, and purposes, suggests a need for multi-level geospatial information to support improved decision making and to enable modeling of potential impacts on social systems (Aubrecht et al. 2012a).

\subsubsection{Mapping exposure for assessing populations at risk}

As stated above, exposure is a core component of disaster risk, without which there would be no impacts from hazards. Also, for some types of natural hazards (tectonic), risk can only be significantly reduced by decreasing exposure and vulnerability, as the hazard component of the equation is quite unresponsive to human intervention. For example, earthquakes and volcanic eruptions are still difficult to predict and we cannot reduce the magnitude of events. Therefore accurately modeling, mapping, and quantifying population exposure becomes an especially critical first step for supporting DRM and DRR.

In exposure modeling and analysis, population distribution maps (grids) are important for the estimation of population exposure, and, in addition, these maps (grids) are often used to model the distribution of other socio-economic and exposure assets, such as GDP (e.g. Jongman et al. 2012). Also, population density is a relevant indicator of (social) vulnerability to hazards (UNDRR 2019). 
Typically, the analysis and quantification of population exposure to hazards can follow a deterministic or probabilistic approach. Deterministic approaches typically quantify maximum potential exposure for a single "what if" hazard scenario (often worst case), for lack of magnitude-frequency information. The increased availability of such information has enabled a progressive shift towards probabilistic assessment, whereas 'physical population exposure' can be computed as the average annual population exposed to a hazard, when the annual frequency of a given magnitude event [event/year] is known (Peduzzi et al. 2009a).

As well as hazard, exposure is dynamic, varying at different spatial and temporal scales. Regarding the dynamics of exposure, some authors distinguish between chronic exposure and acute exposure as an extension of their concept of acute and chronic hazards (Tobin et al. 2011). In this proposal, an 'acute hazard' is "a sudden-onset event with a limited duration (typically weeks or less) and not regularly repeated", whereas a 'chronic hazard' is "an event that is of unforeseen duration (e.g., volcanic activity) or that is regular/repeated (e.g., yearly floods)" (Tobin et al. 2011, pp. 701).

Evaluating population exposure for global disaster risk assessment is challenging, being limited by the availability and quality of geophysical and socio-economic data (Lerner-Lam 2007; Peduzzi et al. 2009a). In fact, estimations of human exposure to hazards have been mostly conducted for limited areas, from parts of cities to small regions (e.g. Wieland et al., 2012; Fraser et al. 2014; Yuan et al. 2019). When such studies were conducted for large areas, such as continents or the globe, they usually did not incorporate a temporal dimension (e.g., Small and Naumann 2001; Dilley et al. 2005). However, analyzing past and present variation of exposure enables identification of dynamics and trends and may provide insight into future changes in risk. Given high urbanization rates and increasing spatio-temporal variability in many present-day cities, exposure information is often out-ofdate, highly aggregated or spatially fragmented (Wieland et al. 2012).

Early efforts at assessing global exposure provided estimates of human exposure to drought, earthquakes, floods, and tropical cyclones (UNDP 2004). These efforts increased in the present decade, with a number of global (e.g. GAR) and international initiatives aimed at DRR producing global exposure databases containing information on settlements and population distribution. These include the Global Exposure Database (GEG) initially developed for GAR 2013, reporting population from LandScan 2007 at $5 \mathrm{~km}$ spatial resolution (De Bono and Mora 2014). For GAR 2015, Pesaresi and Freire (2014) developed and provided an improved global grid reporting the percentage of built-up areas per 30 arc-second cell. For GAR 2019, the exposure model has been improved with more accurate measurement tools (UNDRR 2019). Developed 
for and in the frame of GED4GEM ${ }^{5}$, the Global Exposure Database (GED) contains aggregate information on population and the number/built area/reconstruction cost of residential and non-residential buildings at a $1 \mathrm{~km}$ resolution.

These global databases have been mostly aimed at baseline risk assessment and inter-country comparison, with their 'operational' value for subsequent DRM phases being very limited due to coarse spatial resolution 6 , lack of temporal component, and lack of consistency. For example, the United States Geological Survey (USGS) estimates population exposure to significant global earthquakes which occurred since 1973 (Allen et al. 2009). However, the analysis departs from a static representation of population distribution, i.e., a layer representing a single date. Improving retrospective analyses of human exposure requires not only spatially explicit demographic data (NRC 2007), but also population datasets having an historical dimension and consistent modeling approach that enable their comparison (e.g. Freire et al. 2015c).

Other global platforms have been developed to support disaster risk and impact assessment of hazards by quickly estimating human exposure to specific hazards. The USGS' PAGER ${ }^{7}$ (Prompt Assessment of Global Earthquakes for Response) system innovated in 2007 by immediately estimating after an event the number of people and settlements exposed to shaking by using the coarse 'ambient' population distribution surface from LandScan (Dobson et al. 2000). Other such operational global platforms and services include:

- INFORM ${ }^{8}$ : Index for Risk Management, provides worldwide national and selected sub-national (baseline) risk indices for management of humanitarian crises and disasters, using average annual population exposed to several hazards (earthquakes, floods, surge from tropical cyclones, tsunamis), being updated annually;

- GDACS ${ }^{9}$ : Global Disaster Alert and Coordination System, providing early estimates and projections of potential population exposure to actual disaster events and their impacts (e.g. Hurricane Dorian, Aug. 2019) (GDACS 2019);

\footnotetext{
${ }^{5}$ https://storage.globalquakemodel.org/what/physical-integratedrisk/exposure-database/

${ }^{6}$ One serious side effect of coarse spatial resolution is that entire nations are not 'gridded' and hence not represented at such scales; this often includes the most exposed to certain hazards, such as small Island Nations (e.g. cyclones, sea level rise).

7 https://earthquake.usgs.gov/data/pager

8 https://drmkc.jrc.ec.europa.eu/inform-index

${ }^{9}$ https://www.gdacs.org
} 
- Copernicus EMS ${ }^{10}$ : rapid mapping prior, during, and after disaster events, mapping areas affected and assessing initial impact of events (including population exposed).

On the ground, three major disasters highlighted limitations in DRM and gaps in population exposure modeling: 2004 Indian Ocean Tsunami - lack of early warning/evacuation, and of modeling the presence of non-residential population in affected areas (i.e. tourists) that composed a large and unprecedented share of the victims; 2010 Haiti Earthquake - lack of detailed and updated geospatial data on built-up and population, prompting massive efforts at 'rapid mapping' via VGI/crowdsourcing; 2011 East Japan Earthquake and Tsunami - the possibility of major NATECH cascading event was overlooked.

One persistent challenge for global DRM is thus "to find innovative, efficient methods to collect, organize, store and communicate exposure data on a global scale, while also accounting for its inherent spatio-temporal dynamics" (Pittore et al. 2017). While until recently, global scale was synonymous with coarse resolution for exposure data, that has been rapidly changing due to developments in geospatial technologies (including information extraction methods) and data availability and access, in particular that derived from Remote Sensing. These enhancements are making more advanced exposure models possible (UNDRR 2019), and even in data rich environments, the use of bottom-up approaches can further improve and update detailed data on human and structural exposure (Freire et al. 2011a).

While many current threats and hazards have a worldwide scope and large disasters can affect entire regions or countries, impacts are fundamentally local, affecting people where they live and conduct their daily activities. Riskrelated policies may be decided at high administrative levels (e.g. national, provincial), but disaster risk reduction and mitigation demand measures implemented at local level, which requires understanding of vulnerabilities at compatible scales (Lerner-Lam, 2007). This is also valid for the preparedness phase, especially in remote locations, as disaster risks have local and specific characteristics that should be understood (UNISDR 2015a). Concerning the assessment of human exposure and DRM activities, this implies the need for more detailed population data capable of supporting local scale analyses and decision-making ${ }^{11}$ (Freire 2010).

Given the obvious social relevance of DRR, this need has been a major driver of research efforts to improve population modeling and related DRM

\footnotetext{
10 https://emergency.copernicus.eu

11 Nonetheless, there are significant challenges and trade-offs affecting the modeling, mapping, and assessment of population exposure. These are essentially related to the modeling of population distribution discussed in section 1.2.4.
} 
applications, especially at national, sub-national, and local scales. These efforts have increased spatio-temporal resolution and therefore accuracy of population exposure assessments demonstrated for a number of hazards, including bombing/explosion (Ahola et al. 2007), tsunami (Taubenbock et al. 2009; Freire et al. 2011b), coastal storm surge (Kellens et al. 2012; Aubrecht et al. 2015), river flooding (Smith et al. 2016; Renner et al. 2018), volcanic hazard (Bhaduri et al. 2002; Freire et al. 2015b), earthquake (Taubenbock et al. 2008; Freire \& Aubrecht 2010; Osaragi 2016), and heat waves (Hu et al. 2019). Hu et al. (2019) have innovated by tackling a hazard displaying significant variation in the daily cycle and coupling with the respective dynamics of population for improving spatio-temporal characterization of human exposure in the city of Chicago, USA.

For support to local-level analyses and actions, baseline population exposure should be ideally conducted at the level of individual buildings, as a focus of human life where most human activities take place (per CAPRA ${ }^{12}$ exposure levels). However, higher detail may be useful for specific hazards and large buildings, as demonstrated in study of tsunami threat in Lisbon (Freire et al. $2011 a ; 2012)$. In the context of river flooding risk and actual impacts, Freire et al. 2015c have demonstrated (i) the importance of producing and using high resolution exposure data for more accurate DRM, and (ii) how recent advancements in geospatial data and modeling enable attaining wide coverage with high spatial resolution, approaching the local scale. This work illustrates and improves a common problem in spatial DRM: the scale mismatch of hazard and exposure data, whereas hazard maps are frequently of higher resolution than exposure data, creating problems for the correct assessment of exposure.

Different types of hazards such as earthquakes, tsunamis, landslides, or fires display different characteristics whose consideration is important for exposure assessment and risk analysis and more broadly for DRM ${ }^{13}$ (Aubrecht et al. 2013). Hazard types vary in regards to their main causal processes (natural vs. man-made), their geographical impact, and timescale: onset and impact duration (Figure 1). But also important for risk analysis are their recurrence intervals or return periods (see 'physical population exposure' above), as changes in the hazard frequency and timing of hazard occurrence during the year will have a strong impact on the ability of societies and ecosystems to cope and adapt to changes (Cardona et al. 2012).

Modeling and mapping population exposure is especially important for those hazards whose element most under direct threat is typically life or health (e.g. tectonic, technological accidents, conflict), while some other hazards directly threaten food or water on which ultimately humans depend.

12 https://ecapra.org

13 This section introduces the topic of role of hazards characteristics, which is further discussed throughout this thesis when relevant. 


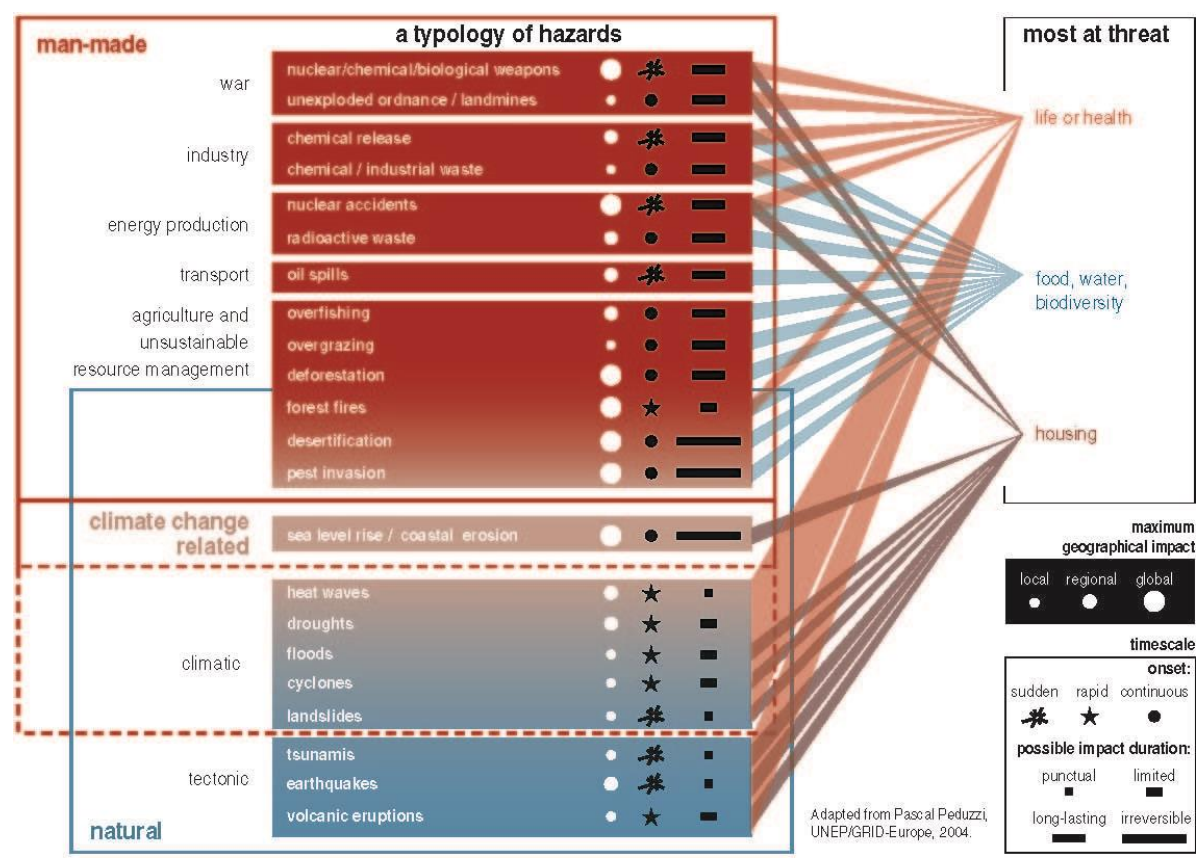

Figure 1 A typology of hazards and respective characteristics ${ }^{14}$. Source: UNEP/GRID Arendal (http://maps.grida.no/go/graphic/typology_of_hazards).

Particularly relevant for the current discussion are hazards' potential impacts, which can occur at spatial and temporal scales that span several orders of magnitude (Gill and Malamud 2014). For example, while ground collapse typically affects very localized areas in the span of seconds to minutes, climate change has a global scope that spans from decades to millennia (Gill and Malamud 2014).

Regarding the temporal scale, human dynamics in the short term (daily and weekly patterns) can strongly affect the quantification of human exposure in cases of extreme natural events with rapid onsets, such as earthquakes, landslides or tsunamis (Pittore et al. 2017). Not accounting for socioeconomic dynamics in exposure and risk assessment, especially in case of time-specific or future events, causes mismatches in temporal scales (Lorenzoni et al. 2000; Rohat 2018). Therefore sudden or rapid hazards are those for which analysis stands to benefit the most from increased temporal resolutions of population exposure and consequently such hazards have driven most recent research efforts to improve population modeling, as mentioned above. Conversely, slow onset hazards (e.g. droughts) are more tolerant to the use of a single

14 Other classifications of hazards exist, such as Integrated Research on Disaster Risk, 2014. Peril Classification and Hazard Glossary (IRDR DATA Publication No. 1). Beijing: Integrated Research on Disaster Risk. 
temporally-averaged measure of population for baseline exposure, such as 'residential' or 'ambient' population (discussed in more detail in section 1.2.3).

Concerning the spatial characteristics and scale, hazard zones of river floods or tsunami are typically much more specific (due to close dependence on topography) and have a 'hard' edge, requiring compatible detailed and precise data for exposure and DRM; on the other hand, other climatic hazards (cyclones, droughts) are less spatially specific and display 'soft' edges, tolerating exposure data having lower spatial resolution and precision. Sudden and highly localized hazards such as landslides or tornadoes are space- and time-specific events which are especially stringent regarding the characteristics of population data to support exposure analysis and DRM, requiring detailed exposure data in both space and time.

Hazards also vary in complexity. Volcanic eruptions are especially complex phenomena capable of causing human casualties from several different hazards and cascading effects (Chester et al. 2000; Brown et al. 2017). Cascading disasters, especially if affecting the same area, are capable of inducing dramatic changes to population distribution and exposure during the development of the events, becoming especially challenging for modeling of human exposure.

In brief, conducting spatially-explicit risk assessment requires modeling and mapping population exposure with sufficient resolution. Assessing potential or actual population exposure to hazards requires geo-information on population distribution at a range of spatial and temporal scales. As stated in GAR2019, "disaggregated data sets and statistical data, previously scarce in the disaster risk realm, are now becoming prerequisites for measuring risk-informed sustainable development; further improvements in data availability, quality and accessibility are anticipated" (UNDRR 2019).

\subsubsection{Beyond exposure: the relevance of population in integrated DRM}

Disaster Risk Reduction (DRR) is the outcome of effective (integrated) Disaster Risk Management (DRM). DRM generally refers to "the application of disaster risk reduction policies and strategies to prevent new disaster risk, reduce existing disaster risk and manage residual risk, contributing to the strengthening of resilience and reduction of disaster losses" (UNISDR 2017). Disaster Risk Management ${ }^{15}$ is a complex multi-scale and multidisciplinary activity involving many actors and stakeholders, and as planning framework for DRR involves multiple dimensions of social and natural systems and their interactions (Neal 1997).

15 Referred to as "Emergency Management" in some geographical contexts, e.g. in USA and Australia. 
DRM has been commonly regarded as a multi-stage process (Johnson 1992; Mileti 1999), conceptualized as a cycle or a spiral (Figure 2) (Aubrecht et al. 2013; van Westen 2013). The process typically comprises the main interconnected phases/ components of (i) planning or risk analysis, (ii) mitigation, (iii) preparedness, (iv) response, and (v) recovery and rehabilitation $^{16}$ (see Lettieri et al. 2009 for a review).

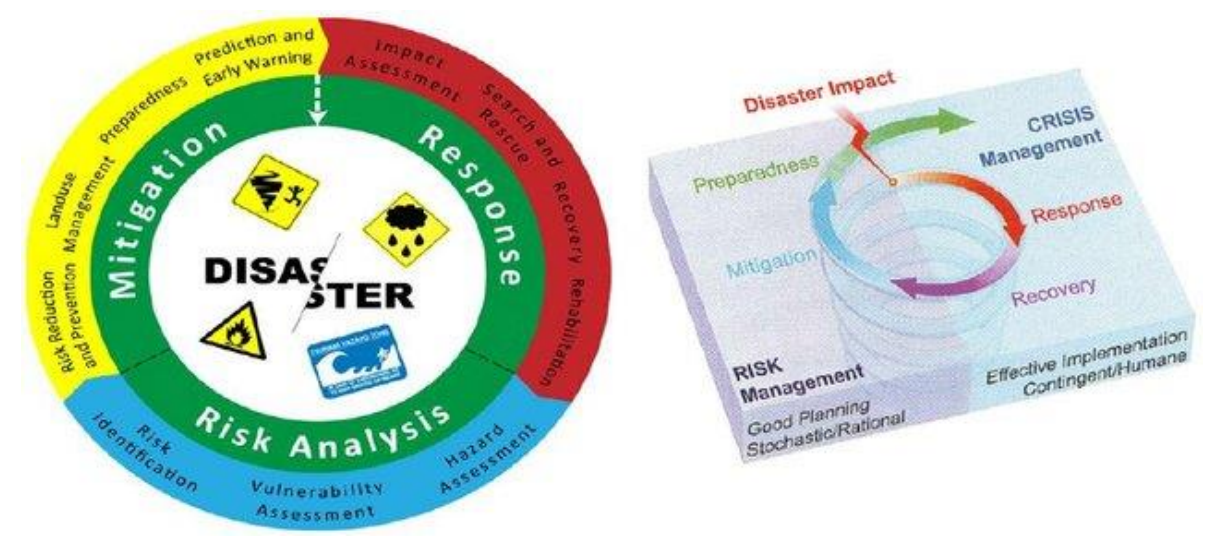

Figure 2 DRM process and phases, conceptualized as cycle and spiral. Source: Aubrecht et al. 2013

One advantage of unrolling the cycle into a spiral is highlighting the need to adopt and 'repeat' a structured approach to DRM through time, that progressively shifts the focus from response and recovery to prevention and preparedness (van Westen 2013) - or from reacting to disasters to preventing them. What is often overlooked is the need to re-analyze risk after mitigation measures are implemented, in order to assess to which extent risk was mitigated and to quantify the remaining (or residual) risk. Aiming at standardization, the recent ISO 31030 (ISO 2018) provides a general approach to the risk assessment process, comprising three stages: risk identification, risk analysis and risk evaluation.

Regardless of the conceptualization of DRM, throughout this process spatial information has a crucial role (van Westen 2013), and this is especially true regarding population. In people-centric DRM, (updated and detailed mapping of) population distribution data is relevant for decision support in (practically) every phase of the disaster risk management cycle (Freire, 2010), if produced at appropriate spatial and temporal scales (Sutton et al. 2003). Since human beings are the most vital element to protect, adequate planning, mitigation, and reaction to disasters requires knowing the location of people and their

16 The number, sequence, and designation of DRM phases have been a matter of proposals and debate among researchers and practitioners, but such discussion is beyond of the scope of this thesis. 
characteristics. Table 1 shows in a non-exhaustive way the usefulness of population geospatial demographic data in each phase of DRM, including its potential role and type of questions addressed ${ }^{17}$.

Table 1 Usefulness of geospatial demographic data (with focus on total population counts and density) in DRM phases. Source: author own elaboration

\begin{tabular}{|c|c|c|}
\hline DRM phases & $\begin{array}{c}\text { Role of geospatial } \\
\text { population data (total } \\
\text { pop.) }\end{array}$ & $\begin{array}{c}\text { Questions } \\
\text { addressed/answered }\end{array}$ \\
\hline \multirow{5}{*}{$\begin{array}{lr}\text { Planning } & \text { (Risk } \\
\text { analysis } & \& \\
\text { assessment) } & \end{array}$} & $\begin{array}{l}\text { Mapping of (potential) } \\
\text { population exposure; }\end{array}$ & $\begin{array}{l}\text { Where are the potentially } \\
\text { exposed people? }\end{array}$ \\
\hline & $\begin{array}{l}\text { Assessment/quantification } \\
\text { of population exposure; }\end{array}$ & $\begin{array}{l}\text { How many people are potentially } \\
\text { exposed? }\end{array}$ \\
\hline & $\begin{array}{l}\text { Identification of human } \\
\text { exposure hot spots; }\end{array}$ & $\begin{array}{l}\text { What are their densities, are } \\
\text { there exposure hotspots? }\end{array}$ \\
\hline & $\begin{array}{l}\text { Visualization, } \\
\text { communication of } \\
\text { distribution of human } \\
\text { exposure for risk awareness; }\end{array}$ & \\
\hline & $\begin{array}{l}\text { Establishing a baseline } \\
\text { situation for assessing risk. }\end{array}$ & \\
\hline \multirow[t]{3}{*}{ Mitigation } & $\begin{array}{l}\text { Assessment and planning of } \\
\text { mitigation measures; }\end{array}$ & $\begin{array}{l}\text { Where are mitigation measures } \\
\text { most needed? }\end{array}$ \\
\hline & $\begin{array}{l}\text { Preparation of population } \\
\text { awareness and self- } \\
\text { protection campaigns; }\end{array}$ & $\begin{array}{l}\text { Is there a need for } \\
\text { relocation/resettlement } \\
\text { population? }\end{array}$ \\
\hline & $\begin{array}{l}\text { Location and quantification } \\
\text { of people targeted for } \\
\text { mitigation measures; }\end{array}$ & $\begin{array}{l}\text { How many people will be affected } \\
\text { by mitigation actions? }\end{array}$ \\
\hline \multirow[t]{5}{*}{ 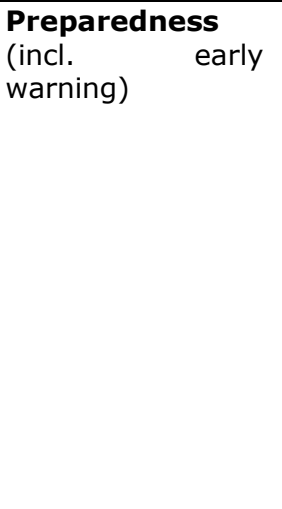 } & $\begin{array}{l}\text { Informing the placement of } \\
\text { means and resources } \\
\text { according to potential }\end{array}$ & $\begin{array}{l}\text { Where and how many supplies } \\
\text { are needed in case of hazardous } \\
\text { events (e.g. water \& food)? }\end{array}$ \\
\hline & vulnerabilities; & $\begin{array}{l}\text { Where should shelters be } \\
\text { located, and what should be their }\end{array}$ \\
\hline & Indicating to whom and & capacity? (e.g. for tsunami) \\
\hline & warning; & $\begin{array}{l}\text { How many people should be } \\
\text { evacuated and to where? What } \\
\text { are the evacuation routes and } \\
\text { their capacity? }\end{array}$ \\
\hline & & $\begin{array}{l}\text { Where to locate personnel and } \\
\text { resources for best response? } \\
\text { (e.g. firemen, ambulances) }\end{array}$ \\
\hline
\end{tabular}

17 Regarding their relation with a specific hazardous event, Planning, Mitigation, and Preparedness are pre-impact, while Response and Recovery are post-impact activities. 


\begin{tabular}{|c|c|c|}
\hline \multirow[t]{3}{*}{ Response } & \multirow{2}{*}{$\begin{array}{l}\text { Locating and estimating } \\
\text { affected people (victims) is } \\
\text { essential to tailor response } \\
\text { and rescue efforts; }\end{array}$} & \multirow{3}{*}{$\begin{array}{l}\text { How many people were affected } \\
\text { and where are they? } \\
\text { How can they be reached and } \\
\text { rescued? } \\
\text { How many potential victims? }\end{array}$} \\
\hline & & \\
\hline & $\begin{array}{l}\text { Location and quantification } \\
\text { of people potentially } \\
\text { affected; Matching of } \\
\text { resources required for } \\
\text { effective response; }\end{array}$ & \\
\hline \multirow[t]{2}{*}{$\begin{array}{l}\text { Rehabilitation } \\
\text { and Recovery }\end{array}$} & \multirow[t]{2}{*}{$\begin{array}{l}\text { Estimating all activities and } \\
\text { people affected, even if } \\
\text { indirectly, facilitates the } \\
\text { recovery process; }\end{array}$} & $\begin{array}{l}\text { How was the baseline (pre- } \\
\text { event) population distribution } \\
\text { and how can that be recovered or } \\
\text { improved upon? }\end{array}$ \\
\hline & & $\begin{array}{l}\text { How to build back better, } \\
\text { planning settlements to reduce } \\
\text { and mitigate future risk? }\end{array}$ \\
\hline
\end{tabular}

Although not exhaustive, Table 1 shows that geospatial population data has a useful role to play in all phases of DRM and that it can potentially provide answers to a range of relevant questions, informing decision-making and action. It becomes clear some of the many ways geospatial population data can contribute to baseline risk analysis and potential impact assessment, mitigation activities, preparedness measures (including early warning and evacuation), response, and rehabilitation (Freire, 2010).

In integrated DRM, Planning should be the necessary (but not sufficient) initial phase, determining the need for and informing specific actions required in subsequent phases. Central to the Planning phase is Risk analysis, which includes the identification of risk and its quantification. The mapping and assessment of population exposure and potential impacts should have a central role in informing actions on the ground, required in sub-sequent phases.

Analysis of pre-event population distribution is necessary for establishing a base-line situation for assessing risk, and pre-event maps are often needed during the response phase (Zerger and Smith, 2003). In the Mitigation phase, geospatial population data can help locate, quantify and rank areas targeted for measures. During Preparedness, these data can inform the placement of means and resources according to potential exposure of people and their vulnerabilities, and indicate to whom and where to direct early warning.

Despite the importance of Mitigation and Preparedness phases in reducing impacts of hazards, it is usually Risk Analysis and Response that receive the most attention. Response requires population totals that are spatially disaggregated for estimating the number of casualties and conducting safety and emergency relief (Tenerelli et al. 2015). 
While DRM is inherently a spatial problem (Goodchild 2006), after a disastrous event the focus quickly turns to time, concerning both the time at which the event has occurred or started to unfold and the so-called 'golden hour' for search and rescue operations (Zerger and Smith 2003; Goodchild 2006). The emergency of the response phase is a strong argument recommending that suitable population distribution data be produced beforehand or rapidly computable on-demand.

The frequent lack of such suitable data leads to the need for rush mode mapping of both pre-event situation and affected areas post-disaster (as in Copernicus EMS) and collaborative mapping via crowdsourcing/VGI, such as HOT-Humanitarian OpenStreetMap ${ }^{18}$ and MapAction ${ }^{19}$. In this respect, the 2010 Haiti earthquake can be considered a landmark event (Norheim-Hagtun and Meier 2010).

Despite efforts at devising efficient population estimation techniques that could be employed in real-time once a disaster occurs (Dobson 2003, 2007), for planning and simulation purposes and to ensure a timely response, adequate population distribution data should be produced and made available beforehand whenever possible. This is especially true for sudden and rapid onset hazards such as those discussed in this thesis (earthquakes, tsunamis, volcanic eruptions) where emergency responders and exposed population are typically caught by surprise or where lead-time is very short.

Another pressing challenge for the DRM/DRR community is the provision of free and open models and data, as "dissemination of exposure data is the key to empowering end-users and communities and for allowing and encouraging risk evaluation" (Pittore et al., 2017), leading the UN to cultivate and promote the generation and use of open data for DRM (GFDDR 2014), as that provided in PREVIEW Global Risk Data Platform ${ }^{20}$.

\subsubsection{Background: Modeling and mapping of population distribution in space and time (for exposure analysis and DRM) ${ }^{21}$}

For modeling, mapping, and assessing human exposure (and in general as support to DRM) population data is a basic necessity, with its quality and level of detail having a direct effect on response and lives saved (NRC 2007). The NRC states that is "preferable in every situation to have [demographic] data at finest possible spatial resolution, especially when dealing with emergency situations" (NRC 2007, p. 31). In the above described context, the need to

\footnotetext{
18 https://www.hotosm.org

19 https://mapaction.org

20 https://preview.grid.unep.ch

21 Parts of this section are based on Leyk et al. 2019. The spatial allocation of population: a review of large-scale gridded population data products and their fitness for use. Earth Syst. Sci. Data, 11, 1385-1409, of which I am co-author.
} 
map population distribution suitable for assessing human exposure to hazards has recently been growing in attention and importance.

The way population data is collected and represented in risk assessment has typically depended on the scale of analysis and the availability of information (Rhind 1991) (in van Westen et al 2013). Census campaigns and other ground surveys are usually the main source of population/demographic data. In georeferenced censuses, counts of resident population are assigned to census reporting units or zones, which vary widely in shape, size, and population densities. While population distribution in space is discontinuous and heterogeneous, maps purely based on census data assume (i) an exhaustive distribution (i.e. there is population in the whole census zone), and (ii) a homogeneous distribution (i.e. density is constant in each zone). Collection of high-quality census data involves significant cost and technical expertise, and such data is usually updated every ten years with longer and irregular updates in many countries (UNDESA 2009). These and other challenges contribute to make the quality, detail, age, and reliability of census data quite heterogeneous among countries (Balk et al. 2006; Leyk et al. 2019). Such shortcomings propagate to derived population grids and their applications.

For accurate spatio-temporal modeling of population distribution, geospatial census data comprise mainly two types of challenges/issues. One is more conceptual, tied to the complex nature of population as a geographical variable (i.e. a discrete quantitative variable, having finite and known counts), the semantical concept (definition of 'residential' population ${ }^{22}$ ) and technical specifications (enumeration vs. reporting units, their reference period, scale, etc.); and another is more practical, tied to the quality and reliability of data. While the first challenge and how to overcome it has received considerable attention by applied research, the latter one has remained largely ignored in production of global population grids and we have addressed it in Chapter 5.

Geospatial modeling allows to overcome or mitigate some of these characteristics and limitations and to address main requirements and challenges for increasing the usefulness of geospatial population data (e.g. higher spatio-temporal resolutions, consistency, quality, currency).

However, modeling the spatio-temporal distribution of population has been typically subject to a number of trade-offs between geographical coverage, spatial resolution, temporal resolution, and uncertainty. These trade-offs are mostly due to data availability and quality, and modeling capacity. To illustrate

\footnotetext{
${ }^{22}$ It should be noted that data on resident populations as provided by censuses are themselves a convention (concept of 'usual residence'), whose distribution never occurs at any specific moment in time (de jure census population) or if it does occur (de facto, location at the time of the census) that distribution may not be representative of a different situation or lose meaning in the medium/long term (i.e., a year) (Leyk et al. 2009).
} 
these trade-offs, let us consider the global population represented at world scale, where the globe or planisphere are the only unit or cell (resolution = world). In this unit and for a given reference day of the year we can model or estimate with high certainty the total amount (count) of daytime and nighttime population, as these are essentially the same for both periods and equal the world's population. However, as we 'zoom in' or start to subdivide the planisphere into smaller units, such as continents, countries, and cities (i.e. increasing the spatial resolution), the amount of people in those units starts to diverge between each of those periods (as a function of human dynamics), and uncertainty will increase as a reflex of (i) frequency of movement and their spatio-temporal domain ${ }^{23}$, and (ii) our lack of knowledge to fully model those human activities and their locations.

Regular grids (raster or vector) are now well established and widely used spatial structures to model and report population attributes due to their flexibility and advantages ${ }^{24}$ (Martin and Bracken 1991; Deichmann et al. 2001). Making population data available as fine grids facilitates analyses, increases flexibility and interoperability with other spatial datasets, and mitigates MAUP (Openshaw and Taylor, 1981). This includes easier integration with hazard data, whose spatial footprints do not conform to administrative boundaries (Smith et al. 2016).

Within the concept and spatial resolution adopted, population grids simultaneously estimate and depict two components of population distribution: (i) where people are (i.e. inhabited vs. uninhabited areas) and (ii) how many there are per unit area (i.e. their densities). To accomplish the estimation of both these aspects, population distribution grids rely on two major types of input datasets in their construction: (i) georeferenced population statistics (e.g. census or microcensus) and (ii) geospatial proxies associated to the presence of population at the selected spatio-temporal scale (covariates).

For a given spatial unit, population distribution grids can be produced by disaggregating or gridding available population counts (top-down approach) or by estimating that count at the grid cell level through combining sampling with ancillary data, typically remotely-sensed (bottom-up method) (e.g. Sutton 2001; Harvey 2002; Wardrop et al. 2017). For top-down approaches, obtaining positive values and preserving the total volume of people - Tobler's 'pycnophylactic condition' (Tobler 1979) - are basic requirements to produce realistic representations. For population grids that are generated by

23 Human activity and dynamics have different spatio-temporal patterns, e.g. from high-frequency and spatially confined daily commuting between place of residence and workplace, to lower-frequency and less confined seasonal movement for summer holidays.

${ }^{24}$ However, at local level other spatial units and data structures can be very useful, e.g. 2-D or 3-D vector representation of buildings, as shown in context of tsunami risk in e.g. Freire et al. 2011a; 2012). 
disaggregating available statistics (top-down) while preserving their volume, output accuracy (with respect to ground truth data) is largely dependent on the spatial detail and quality of input geospatial layers (Freire et al. 2018a).

In the spatial refinement of existing population statistics (top-down), many approaches have been tested and used (Wu et al. 2005), but most are variations of the classical dasymetric mapping technique (Semenov-TianShansky 1928; Wright 1936). Dasymetric mapping is a simple yet powerful cartographic technique that allows limiting the distribution of a variable to the zones where it is present by using related ancillary information in the process of areal interpolation (Eicher and Brewer 2001). The technique has been progressively revived and refined in last two decades, namely with development of 'intelligent dasymetric mapping' variants (Mennis 2003; Mennis and Hultgren, 2006).

In dasymetric mapping of population, the quality of the proxy variable(s) is more important than the choice of the downscaling method/algorithm (Martin et al. 2000). Hence, all other factors being equal, the factors determining the output accuracy of the final map (grid) can be ranked as follows:

1. Detail (resolution) and quality of source zoning population (i.e. census statistics),

2. Quality of proxy variable (covariate) for population disaggregation,

3. Specific methodology applied for disaggregation from source zones to target zones.

Then for disaggregating global population grids based on dasymetric mapping (either using one or more covariates as input), the challenge can be narrowed to two aspects: (i) obtaining reliable geospatial population statistics, and (ii) creating an appropriate proxy (target) layer associated to the presence of population at the selected spatio-temporal scale (i.e. covariate). In most population disaggregation approaches, significant effort and cost is expended preparing, combining and modeling geographic variables in order to obtain an appropriate proxy layer or probability surface to support population disaggregation (e.g. WorldPop ${ }^{25}$ ). In developing more detailed multi-temporal global population grids, this thesis (Chapter 5 ) has focused instead on aspects (1) and (2) mentioned above.

\subsubsection{Global Population Grids}

At global level, starting in the 1990 s the increased availability of digital spatial data combined with improved capabilities of Geographic Information Systems (GIS) have enabled the development of several global population distribution grids, such as the GPW (Tobler et al. 1995), HYDE (Goldewijk and Battjes

25 https://www.worldpop.org 
1997), LandScan (Dobson et al. 2000), and GRUMP (Balk et al. 2005) ${ }^{26}$. These grids vary in regards to population concept, data sources, and modeling approach - for a detailed review of current large-scale gridded population data products, see Leyk et al. 2019.

Although global population grids have been available for more than two decades, constraints have limited their usability for some applications (Hay et al. 2005; Linard and Tatem 2012; Mondal and Tatem 2012). Among these are their coarse spatial resolution (maximum was $30^{\prime \prime}$ ), use of dichotomous maps of built-up in their construction, and coarse, limited, and incomplete discrimination of urban areas and settlements (especially smaller ones) (Potere and Schneider 2007; Potere et al. 2009). Better and finer global analyses of population-environment interactions require enhanced geoinformation on population distribution and densities, in particular concerning temporal and spatial resolution and capacity for change assessment. For global change detection and assessment, having a clear and coherent spatio-temporal production model is important to ensure users' confidence and allow comparisons in space and time (Freire et al. 2016a). Because even if conducted at global scale, exposure assessment to some hazards is highly sensitive to spatial resolution of population grids: e.g. tsunamis, river floods, landslides.

In the modeling of population distribution, geo-information science and Earth observation play an increasingly important role (Leyk et al 2019). These technologies have been contributing in several ways to improving population modeling for decades (Tenerelli et al. 2015), but more recent developments in imagery and methods for information extraction have been evolving towards constituting a more detailed, objective and independent data source on human presence on the Earth surface. The combination of new cost-effective, automated and fully replicable data classification methods (e.g. machine learning) with the synoptic capacities of satellite Earth Observation imagery, made accessible in a public, full open-and-free frame, can contribute to fill information gaps. This is especially true in poor, remote, unsafe, disputed, very large, and/or highly dynamic areas of the globe where conventional data gathering and updating is challenging (Freire et al. 2018a).

At global level, these developments have enabled global mapping of built-up areas and settlements with unprecedented spatio-temporal detail, consistency, and temporal coverage - in essence capturing the local scale with global coverage. These new remotely-sensed global datasets include the Global Human Settlement Layer (GHSL) (Pesaresi and Ehrlich 2009; Corbane et al. 2017), mining both optical and radar imagery in the period 1975-2016, and

${ }^{26}$ These were the only global gridded population data products available when work for this thesis was initiated. 
the Global Urban Footprint (GUF) for 2012, generated from radar satellite constellation of TerraSAR-X and TanDEM-X (Esch et al., 2017).

Making these datasets available open and free, as in the case of GHSL, helps to increase access, promotes transparency, and ensures accountability of the information produced. GHSL's enhanced spatio-temporal mapping of buildings and density of built-up areas offers a suitable proxy for the location of people and, as such, is being used for enhancing multitemporal global population grids, such as GHS-POP (covering the period 1975-2015) and WorldPop (covering years 2000-2020), and to create time series of population estimates that can be used to fill in data gaps between national census surveys that are commonly taken at decadal intervals (Nieves et al. 2018). In the case of WorldPop, built-up areas are being used in combination with other spatial covariates to create population grids (Stevens et al. 2015).

\section{Box 1.1 The GHS-POP gridded population time series}

The Global Human Settlement Population Grid (GHS-POP) depicts the distribution and density of the total population as the number of people per grid cell (at 250 m spatial resolution) for nominal epochs 1975-1990-20002015. Using dasymetric mapping, residential population estimates (counts) per smallest census unit available, provided by CIESIN GPWv4 for the years of interest, are disaggregated to grid cells informed by the distribution and density of built-up areas as mapped by GHSL (GHS-BUILT) for matching epochs (Freire et al. 2016a; 2018).

At time of first public release (2016) GHS-POP was the highest resolution gridded global population time series and remains the only detailed such dataset spanning the last 40 years. Respect to previous global time series, GHS-POP innovated by increasing spatial resolution by a factor of 16 (from $\sim 1 \mathrm{~km}$ to $250 \mathrm{~m}$ ) while improving temporal depth respect to other global gridded series (GPW, GRUMP), and by representing both population counts and densities by using an equal-area projection (World Mollweide). It is the only disaggregated global dataset relying on a single, time-specific, and consistent proxy (built-up areas), limiting the risk of circularity and endogeneity in analyses. For latest release, areas declared as unpopulated were revised critically and the representation of population along coastlines was improved (Freire et al. 2018a). In order to increase their usability (Lewis 2014), since 2019 these grids are also provided in two different coordinate systems (addition of WGS84), and can be downloaded as bulk or in regular tiles.

Global mapping of built-up areas was performed through the Global Human Settlement Layer (GHSL) project using Landsat imagery collections for nominal epochs 1975, 1990, 2000 and 2014 (Pesaresi et al. 2013; 2016). The GHSL approach is grounded on the concept that buildings and their agglomerations (i.e., settlements) are nowadays the main visible and direct manifestation of human presence (and activity) on the Earth's surface.

GHS-POP is part of an integrated suite of geospatial products, aiming to constitute a detailed and consistent time series of lightly modeled population distributions that is based on reproducible methods for sustainable data production (Melchiorri et al. 2019) and can be used in policy support in 
numerous domains (Ehrlich et al. 2018a), including monitoring exposure trends (Ehrlich et al. 2018b). These grids are created using consistent, open and free input data integrated using a clear and transparent approach and are also freely accessible and downloadable at https://ghsl.jrc.ec.europa.eu/ghs_pop2019.php (last access: 30 September 2019).

At continental level, new big earth data analytics are also enabling sustained mapping of built-up areas at high spatial resolutions, such as the European Settlement Map (ESM) (Ferri et al. 2014). Detailed mapping of built-up areas (at $10 \mathrm{~m}$ ) from ESM was combined with an enhanced LULC map to disaggregate detailed census 2011 data for producing the most detailed pan-European grid of resident population ( $100 \mathrm{~m}$ ) spanning 43 countries (Freire and Halkia 2014). For several countries a model was developed to increase the spatial resolution of source census data and population was gridded at $10 \mathrm{~m}$, to fully preserve the maximum resolution of census data. Also, a new disaggregation approach was developed with two levels, where land use land cover determines reallocation of population within source zones, and built-up density determines specific densities at the grid cell level. The latter population grids showed promise for improving DRM operations at local scale, namely in frame of Copernicus EMS Mapping 27 activities (Freire et al. 2015c). ESM is now in its third version, with Symbolic Machine Learning (SML) supervised classifier, the PANTEX and the morphological image features being employed to map buildings for 39 countries from multi-sensor very high resolution optical imagery (multispectral and panchromatic) for reference year 2015, totaling 127TB of data (Sabo et al. 2019). An added-value to the previous versions is the improved automatic detection of buildings, automatic extraction of water, extraction of building typology (residential vs. non-residential) and an information layer allowing to derive city indicators (Corbane and Sabo 2019). These data are expected to benefit modeling of population distribution.

Other developments in population and settlement mapping are progressing on a country basis, such as the High Resolution Settlement Layer (HRSL) (Tieke et al. 2019). CIESIN is producing and providing population distribution grids at a resolution of 1 arc-second $(\sim 30 \mathrm{~m})$ for 2015, based on settlement extents extracted from very high-resolution $(0.5 \mathrm{~m})$ satellite imagery by the Connectivity Lab at Facebook, using convolutional neural networks (CNN).

\subsubsection{Increasing temporal resolution and integrating unconventional data sources}

Census figures register where people reside and usually sleep ${ }^{28}$, although their spatial distribution varies widely over daily, seasonal and long term time scales

27 https://emergency.copernicus.eu/mapping/ems/emergency-managementservice-mapping

28 Within the definition of 'usual residence' for resident population concept; resident population is usually taken as an approximation of nighttime 
(Sutton et al. 2003). This variation in both space and time can be seen as the static and the dynamic components of population distribution. In this view the static component typically refers to the number of inhabitants per mapping unit (and their characteristics), whereas the dynamic component results from their activity patterns, and their distribution along the spatial and temporal dimensions (van Westen et al. 2013). Due to the diverse locations of human activities (e.g. work, study, leisure) and the displacements they induce, the spatial distribution of population is strongly time-dependent, particularly in metropolitan areas, characterized by intense commuting flows ${ }^{29}$ (Foley 1952; 1954; Schmitt 1956). However, these high frequency dynamics are often not captured by available population data sources at needed spatio-temporal scales, and typically require geospatial modeling for their representation as spatially-explicit data. Especially for disaster risk management in urban areas, it is the population variation during the daily cycle that is particularly relevant, and in this respect the most important determination is whether an incident occurs at night or during the day (Dobson 2007; Alçada-Almeida 2009).

Incidentally, a number of prominent contemporary disasters occurred during the daytime period, e.g.: 9/11 attacks (USA, 2001) between 8:46 - 10:28 am; 2010 Haiti Earthquake, at 4:53 pm; 2011 Great Tohoku Earthquake and Tsunami, at 2:46 pm (all local times). These events occurred when a large share of the population was in workplaces, schools, etc. This implied that even detailed, accurate and current population distribution maps based on census data (i.e. resident population), if available, were rendered mostly operationally useless for Emergency Management (Response).

Modeling population distribution at high temporal resolution (e.g. day vs. night) is especially challenging regarding the two main tasks or steps involved, i.e. (i) obtaining or estimating stocks of population groups for given zones and time periods, and (ii) disaggregating or allocating these population counts to respective target units or place of activity (Martin et al. 2015). For very localized areas of interest (AOI) and limited temporal events (e.g. a stadium during a football match) this is relatively straightforward, as the problem is limited to mapping the expected population (i.e. attendance volume) present at the event in the area of the event. However, it becomes increasingly challenging as the study area increases, given the need to balance the population budget and correctly subtract the attendance from the surrounding areas it originated from and where it is not present during the event (e.g. using an origin-destination matrix). To further complicate matters, daytime

population, in what is clearly a simplification of a more complex reality of population distribution during that period.

${ }^{29}$ It should be noted that also residential population is subject to dynamics and displacement, and that these can be especially quick and intense in case of conflict or other crises (e.g. in Syria, see Corbane et al. 2016). 
population distribution can be considerably different in a working or a weekend day or holiday.

Twenty years ago, the LandScan project (Dobson et al. 2000) has incorporated the temporal dimension in global population distribution by mapping 'ambient' population ${ }^{30}$, an "average population distribution over a 24-hour period" (Badhuri et al. 2002) that "integrates diurnal movements and collective travel habits into a single measure" (Dobson et al. 2000). Conceptually, by straying away from residence-based population density, this representation may be more adequate for assessing exposure for daytime hazard events or those having slow onset, affecting populations over a wide temporal window (e.g. pollution, epidemics, toxic release). The latter case is especially fitting owing to the low temporal specificity of LandScan Global data. However, LandScan's spatial resolution (30 arc-seconds, $\sim 1 \mathrm{~km}$ at Equator), while acceptable for most global and national analyses, is insufficient for most sub-national requirements. Furthermore, ambient population corresponds to a compromise between daytime and nighttime distributions that strictly represents neither period.

Meeting the need for more temporally-explicit representations, two subsequent population distribution databases having higher temporal and spatial detail were developed for the territory of the USA: a team at Los Alamos National Laboratory mapped day- vs nighttime and indoor and outdoor populations at $250 \mathrm{~m}$ (McPherson and Brown 2004; McPherson et al. 2004), and LandScan USA started modeling day- vs night populations (Bhaduri et al. 2002), then evolving into mapping their distributions also on weekends and special events (Bhaduri et al. 2007). Outside of the US, a few ad-hoc efforts succeeded in providing such important improvements, most at local level and even fewer at national scale: these include models for Helsinki (Ahola et al. 2007), the UK Population 24/7 project (Martin et al. 2010), work for Lisbon (Freire 2010; Freire et al. 2012; 2013), the DynaPop model (Aubrecht et al. 2014), and a series of models for Tokyo (Osaragi 2009; 2016). Such models typically rely on intensive geospatial and/or statistical databases on time usage, which are usually only available for selected areas and often at a high cost. This raises the issue of the sustainability of approaches, an important requirement for continued support to DRM activities. Building on the high resolution European Settlement Map (ESM) built-up layer (Ferri et al. 2014), Freire et al. (2015b) have proposed an approach based on available free data (the 'NDPop' model) enabling the refinement of the spatial distribution of resident population and the addition of a temporal dimension to population data (day- vs night), wherever a LULC map is available (e.g. whole Europe). However, in this model

30 Despite this distinct definition, the concept is commonly abused in scientific literature as representing 'residential' population. 
the discrimination of daytime population is dependent on the quality of LandScan global grid of ambient population.

In recent years, a number of emerging data sources and technologies were explored for direct mapping of population or as alternative proxies for its disaggregation, but mainly as proof-of-concept. These include mobile phones (Deville et al. 2014), crowdsourcing/Volunteered Geographic Information (VGI) (Bakillah et al. 2014), and location-based social media (LBSM) (Aubrecht et al. 2011; 2017). LBSM appears especially valuable for dynamic population distributing modeling by providing information both on time usage and on target zone characterization (Aubrecht et al. 2017a). However promising, a number of issues involve these types of data and technologies, e.g. sustainability of approaches, data access and ownership, privacy and anonymity of social media users, and representation bias (Zhang and Zhu 2018). The main challenge for developers and users is how to scale up highly localized approaches to wide geographical areas (continents, world) and provide datasets open and free.

Considering the trade-offs mentioned above, mapping the dynamics of population is especially challenging for large, multi-national areas and typically involves intensive work of identifying, mining, combining and matching heterogeneous statistical and spatial data from disparate sources. This requires significant investment in data integration and harmonization, to improve and supplement limitations of single remote sensing and conventional map sources (Lloyd et al. 2019). The combination of conventional (remote sensing data, LULC maps) with unconventional big geospatial data sources (VGI, POIs) is allowing overcoming some of the previous challenges and gaps, such as improving accuracies, increasing temporal resolution, while covering wide geographical areas (Ye et al. 2019; Yang et al. 2019). The project ENACT ${ }^{31}$ (ENhancing ACTivity and population mapping) succeeded in producing the first continent-wide (28 countries of EU), consistent, seamless, high-resolution and validated population density grids that take into account both daily and seasonal variations: day- and nighttime grids per month of the year were produced at $1 \mathrm{~km}$ resolution ${ }^{32}$, for 2011 (Batista e Silva et al. 2018), accounting for workers, students, and visitors. This effort was aided by technical advances and increased availability and access to various big data sources that enabled the detailed mapping of land use and activities (Rosina et al. 2018). In the context of risk analysis and DRM, we have combined the ENACT population

31 https://ghsl.jrc.ec.europa.eu/enact.php

32 Although in ENACT it was possible to decrease the trade-off between geographical coverage and temporal resolution, there remains the typical trade-off between spatial and temporal resolution: although the latter resolution has increased 24 -fold, this was only possible at $1 \mathrm{~km}$ spatial resolution, or 100 times lower detail than previous best-available PanEuropean resident population grids (100 m), by Freire and Halkia (2014). 
grids with the most recent pan-European hazard data on flood and seismic hazard, in order to map and quantify variations of population exposure, to study their spatio-temporal patterns, and to identify potential daily and seasonal exposure hot spots (Freire et al. 2018b; Schiavina et al. 2018).

In brief, for effective support to DRM, geospatial population data should be accurate, trusted and reliable (UNDRR 2019). Furthermore, it should be up-todate, have sufficient resolution (spatial, temporal, thematic), and be readily available (i.e. produced beforehand or rapidly computable on-demand) through sustainable and reproducible methods. Such population data are still lacking for many countries and regions, both rich and poor, and conducting DRM at global scale would benefit from complete, consistent, and integrated datasets.

\subsection{Objectives and structure of the Dissertation}

The mapping of human distribution and population exposure has generally lagged behind hazard modeling and mapping, in terms of accuracy, detail, and currency. The main objective of this research was to develop methods to improve the mapping of population distribution across multiple spatio-temporal scales in support of Disaster Risk Management. This thesis comprises contributions of population distribution modeling to advancing Disaster Risk Management and Reduction efforts by:

(i) developing several geospatial models that improve (in detail, currency, quality) representation/mapping of population distribution at a range of spatial and temporal scales (Chapters 2, 3, 5),

(ii) applying those data to real disaster risk scenarios for different hazard types by combining geospatial population layers with hazard maps (Chapters 2, 3, 4, 6),

(iii) using spatial analysis for assessing human exposure, including quantitative and qualitative analyses, map representations and visualization (Chapters 2, 3, 4, 6).

We progress from local to global coverage, following specific modeling capacity and requirements at local level with increasing ability to bring improvements to the global domain. The research supporting this work, still ongoing, has taken place over a period of 9 years (concurrent with other research activities and topics) and has so far comprised more than 50 scientific and technical publications. The five core chapters are published as peer-reviewed publications, four of them as articles in different ISI-indexed journals.

With each model development and application, we aim to contribute to answering a set of questions, as presented in Error! Reference source not $\mathbf{f}$ ound.. This table also shows the overall objectives and the range of different issues addressed in each chapter. 
Table 2 Overview of Chapters in this Thesis

\begin{tabular}{|c|c|c|c|}
\hline Chapters & Objectives & Key Questions & $\begin{array}{c}\text { Issues } \\
\text { Addressed }\end{array}$ \\
\hline $\begin{array}{l}\text { 2. Modeling of } \\
\text { spatio-temporal } \\
\text { distribution of } \\
\text { urban population } \\
\text { at high-resolution } \\
- \text { value for risk } \\
\text { assessment and } \\
\text { emergency } \\
\text { management }\end{array}$ & $\begin{array}{l}\text { To demonstrate } \\
\text { and discuss the } \\
\text { value for risk } \\
\text { assessment and } \\
\text { emergency } \\
\text { management of } \\
\text { modeling the } \\
\text { distribution of } \\
\text { urban population } \\
\text { at higher spatial } \\
\text { and temporal } \\
\text { resolutions, } \\
\text { enable local-level } \\
\text { analyses. }\end{array}$ & $\begin{array}{l}\text { Is the spatial } \\
\text { distribution of } \\
\text { urban population in } \\
\text { the daily cycle } \\
\text { relevant for risk } \\
\text { analysis } \\
\text { (exposure) at local } \\
\text { scale? } \\
\text { How can more } \\
\text { detailed modeling } \\
\text { of spatio-temporal } \\
\text { distribution of } \\
\text { urban population } \\
\text { contribute to DRM, } \\
\text { considering its } \\
\text { cycle? }\end{array}$ & $\begin{array}{l}\text { Relevance of } \\
\text { spatially-explicit } \\
\text { population data for } \\
\text { DRM/emergency } \\
\text { management. } \\
\text { Approaches to map } \\
\text { population } \\
\text { distribution } \\
\text { space and time. } \\
\text { Uses and benefits } \\
\text { of detailed day and } \\
\text { nighttime } \\
\text { population } \\
\text { distribution grids } \\
\text { are illustrated for } \\
\text { four } \\
\text { disaster scenarios, } \\
\text { natural and man- } \\
\text { made. } \\
\text { Spatio-temporal } \\
\text { characteristics of } \\
\text { disaster } \\
\text { development for } \\
\text { population } \\
\text { exposure. }\end{array}$ \\
\hline $\begin{array}{l}3 . \quad \text { Integrating } \\
\text { population } \\
\text { dynamics into } \\
\text { mapping human } \\
\text { exposure to } \\
\text { seismic hazard }\end{array}$ & 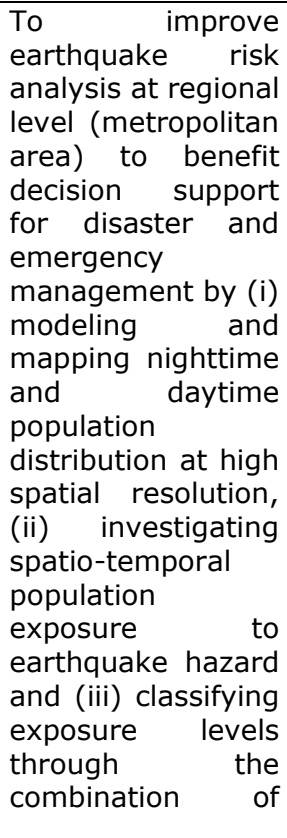 & $\begin{array}{l}\text { Can new geospatial } \\
\text { data on spatio- } \\
\text { temporal of } \\
\text { distribution an } \\
\text { population, derived } \\
\text { by scaling-up a } \\
\text { costly local-based } \\
\text { approach, be } \\
\text { combined with an } \\
\text { earthquake hazard } \\
\text { map to improve } \\
\text { human exposure } \\
\text { mapping and } \\
\text { assessment in a } \\
\text { metropolitan area? } \\
\text { What is the } \\
\text { estimated variation } \\
\text { in population } \\
\text { exposure by } \\
\text { seismic level in the } \\
\text { Lisbon Area } \\
\text { Metropolitan An a }\end{array}$ & 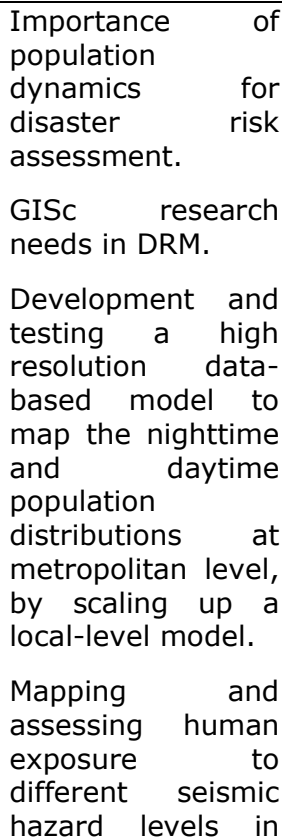 \\
\hline
\end{tabular}




\begin{tabular}{|c|c|c|c|}
\hline Chapters & Objectives & Key Questions & $\begin{array}{c}\text { Issues } \\
\text { Addressed }\end{array}$ \\
\hline & 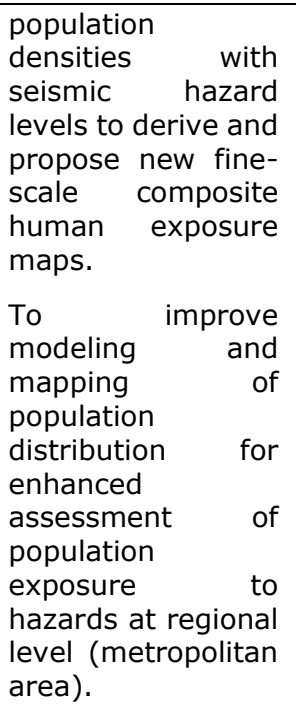 & $\begin{array}{l}\text { (LMA) in day- and } \\
\text { nighttime periods? }\end{array}$ & $\begin{array}{l}\text { day- vs nighttime } \\
\text { periods, } \\
\text { demonstrated with } \\
\text { real seismic } \\
\text { scenario in Lisbon } \\
\text { Metropolitan Area } \\
\text { (Portugal). } \\
\text { Visualization and } \\
\text { communication of } \\
\text { human exposure. } \\
\text { Potential } \\
\text { implications of } \\
\text { results for DRM. }\end{array}$ \\
\hline $\begin{array}{lr}4 . & \text { Advancing } \\
\text { tsunami } & \text { risk } \\
\text { assessment } & \text { by } \\
\text { improving } & \text { spatio- } \\
\text { temporal } & \\
\text { population } & \\
\text { exposure } & \text { and } \\
\text { evacuation } & \\
\text { modeling } & \end{array}$ & $\begin{array}{l}\text { To improve the } \\
\text { assessment of } \\
\text { tsunami risk and } \\
\text { contributing to } \\
\text { more efficient and } \\
\text { effective DRM by } \\
\text { considering the } \\
\text { time dependence } \\
\text { (daily cycle) of } \\
\text { population } \\
\text { exposure and } \\
\text { evacuation from } \\
\text { this hazard in a } \\
\text { metropolitan area. }\end{array}$ & $\begin{array}{l}\text { How is the spatio- } \\
\text { temporal } \\
\text { distribution of } \\
\text { population in the } \\
\text { daily cycle in a } \\
\text { metropolitan area } \\
\text { relevant } \\
\text { population for } \\
\text { exposure to and } \\
\text { evacuation from } \\
\text { tsunami hazard? }\end{array}$ & $\begin{array}{l}\text { Tsunami hazard, } \\
\text { population } \\
\text { exposure, and risk. } \\
\text { Modeling and } \\
\text { mapping } \\
\text { evacuation time in } \\
\text { day and nighttime } \\
\text { periods. } \\
\text { Potential } \\
\text { implications of } \\
\text { results for DRM. }\end{array}$ \\
\hline $\begin{array}{l}\text { 5. Enhanced data } \\
\text { and methods for } \\
\text { improving open } \\
\text { and free global } \\
\text { population grids: } \\
\text { putting 'leaving no } \\
\text { one behind' into } \\
\text { practice }\end{array}$ & $\begin{array}{l}\text { To test data and } \\
\text { methods } \\
\text { mitigate } \\
\text { deficiencies } \\
\text { affecting global } \\
\text { collections of } \\
\text { census data, for } \\
\text { improving open } \\
\text { and free global } \\
\text { population } \\
\text { distribution grids. }\end{array}$ & 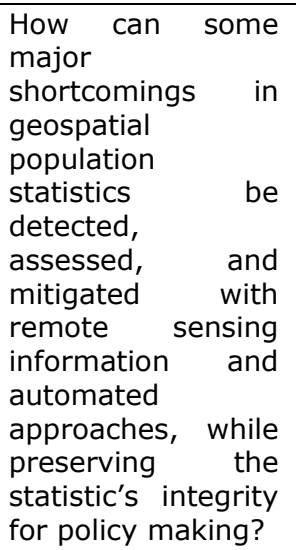 & $\begin{array}{l}\text { Characteristics and } \\
\text { requirements of } \\
\text { population data } \\
\text { and grids for } \\
\text { international policy } \\
\text { support. } \\
\text { Taxonomy of } \\
\text { shortcomings } \\
\text { affecting } \\
\text { geospatial } \\
\text { population } \\
\text { statistics. } \\
\text { Potential sources of } \\
\text { detected anomalies }\end{array}$ \\
\hline
\end{tabular}




\begin{tabular}{|c|c|c|c|}
\hline Chapters & Objectives & Key Questions & $\begin{array}{c}\text { Issues } \\
\text { Addressed }\end{array}$ \\
\hline & & & $\begin{array}{l}\text { and } \\
\text { inconsistencies. } \\
\text { Shortcomings } \\
\text { present in global } \\
\text { collections of } \\
\text { census data, by } \\
\text { introducing, } \\
\text { applying, and } \\
\text { discussing novel } \\
\text { procedures aimed } \\
\text { at investigating } \\
\text { and detecting } \\
\text { some of those } \\
\text { major anomalies. } \\
\text { Automated } \\
\text { detection of some } \\
\text { detected } \\
\text { inconsistencies } \\
\text { using high } \\
\text { resolution } \\
\text { geospatial data } \\
\text { derived from } \\
\text { remote sensing. } \\
\text { Automated } \\
\text { mitigation } \\
\text { inconsistencies of } \\
\text { using the 'split and } \\
\text { merge' approach. }\end{array}$ \\
\hline $\begin{array}{l}\text { 6. An Improved } \\
\text { Global Analysis of } \\
\text { Population } \\
\text { Distribution in } \\
\text { Proximity to Active } \\
\text { Volcanoes, 1975- } \\
2015\end{array}$ & $\begin{array}{l}\text { To characterize } \\
\text { with } \\
\text { unprecedented } \\
\text { detail the spatio- } \\
\text { temporal changes } \\
\text { in the distribution } \\
\text { of human } \\
\text { population in } \\
\text { proximity r to } \\
\text { historically active } \\
\text { volcanoes by } \\
\text { combining detailed } \\
\text { geospatial grids of } \\
\text { global population } \\
\text { densities for the } \\
\text { 1975-1990-2000- } \\
\text { 2015 periods with } \\
\text { the latest data on } \\
\text { distribution of } \\
\text { volcanoes on } \\
\text { Earth, considering } \\
\text { both Holocene } \\
\text { volcanoes and } \\
\text { those having }\end{array}$ & 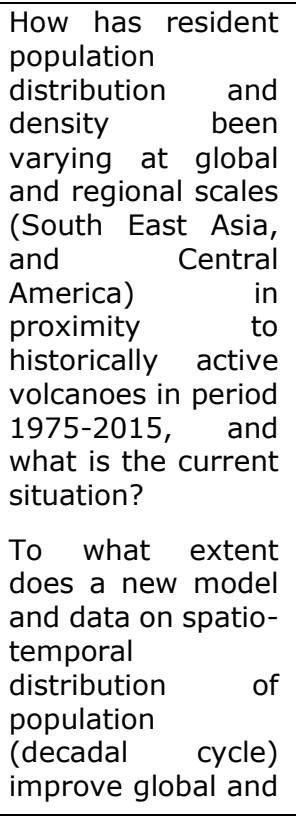 & 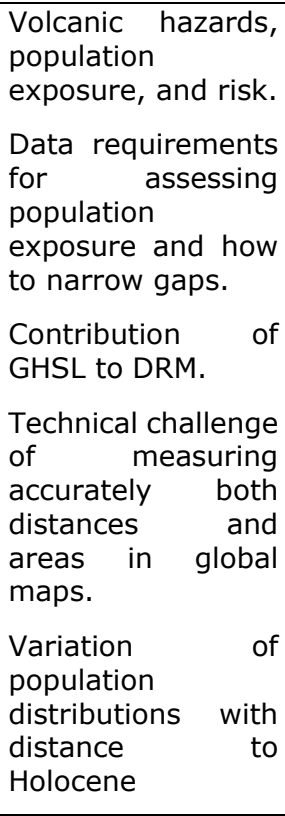 \\
\hline
\end{tabular}




\begin{tabular}{|c|c|c|c|c|}
\hline \multirow[t]{2}{*}{ Chapters } & Objectives & Key Questions & \multicolumn{2}{|c|}{$\begin{array}{c}\text { Issues } \\
\text { Addressed }\end{array}$} \\
\hline & 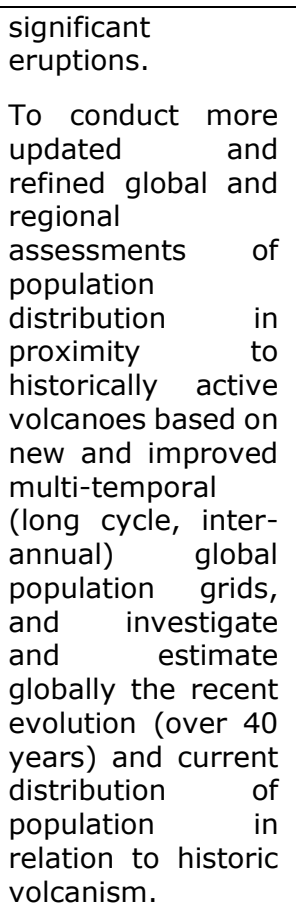 & $\begin{array}{l}\text { regional } \\
\text { characterization of } \\
\text { population living in } \\
\text { proximity to } \\
\text { volcanoes and the } \\
\text { assessment of } \\
\text { population } \\
\text { potentially affected } \\
\text { by their hazards? }\end{array}$ & $\begin{array}{l}\text { volcanoes } \\
\text { those } \\
\text { significant } \\
\text { eruptions, } \\
2015 . \\
\text { Potential } \\
\text { implications } \\
\text { DRM. }\end{array}$ & $\begin{array}{r}\text { and } \\
\text { with } \\
1975- \\
\text { for }\end{array}$ \\
\hline
\end{tabular}

To summarize the chapter contents, Chapter $\mathbf{2}$ sets the stage for the thesis by demonstrating and discussing the value for risk analysis and emergency management of modeling the distribution of urban population at higher spatial and temporal resolutions, to enable local-level analysis. Uses and benefits of detailed day- and nighttime population distribution grids are illustrated for four different disaster scenarios (using fictitious hazard data), either natural and man-made, at municipal level.

Chapter 3 departs from the population distribution model from Chapter 2 and scales it up to regional level (the Lisbon Metropolitan Area) to improve modeling and mapping of population distribution for enhanced assessment of population exposure to earthquake hazard, which is quite relevant in the study area. The chapter discusses how to benefit decision support for disaster and emergency management by (i) modeling and mapping nighttime and daytime population distribution at high spatial resolution, (ii) investigating spatiotemporal population exposure to earthquake hazard and (iii) classifying exposure levels through the combination of population densities with seismic hazard levels to derive and propose new fine-scale composite human exposure maps. This constitutes the first known integration of explicit and detailed 
spatio-temporal population dynamics into mapping human exposure to seismic hazard in a metropolitan area.

In Chapter $\mathbf{4}$ we address improvements to the assessment of tsunami risk and contributions to more efficient and effective DRM by considering the time dependence (in the daily cycle) of population distribution in a metropolitan area, where this hazard is rather significant. In addition to assessing variation of human exposure, we conduct a simulation of evacuation from this hazard in day- and nighttime periods. This work provides a pioneering example of the integration of spatio-temporal population dynamics to assess exposure to tsunami for a whole metropolitan area.

Chapter $\mathbf{5}$ is more policy focused, concerned with characteristics and requirements of population data and grids for supporting requirements of post2015 international development agreements (e.g. SDGs, Sendai) and respective indicators. In this chapter we develop /explore/propose new automated approaches to detecting, assessing, and mitigating some of the major deficiencies present in global geospatial population statistics using remotely sensed data and geospatial modeling.

Finally, in Chapter 6 we use the multi-temporal global population grids enhanced with developments from Chapter 5 to conduct more updated and refined global and regional assessments of population potentially affected by volcanism. Based on these improved multi-temporal (long cycle, inter-annual) population grids, we investigate and estimate globally the recent evolution (1975-2015) and current distribution of population in relation to historic volcanism.

In a concluding chapter (Chapter $\mathbf{7}$ ) we summarize the main contributions of the approaches presented in previous chapters, in relation to respective research objectives and questions, and discuss ongoing and future developments in modeling population distribution to support Disaster Risk Management and Reduction.

All chapters (articles) develop and apply new methodologies to solve problems related to modeling and mapping population distribution and exposure to hazards, aiming to improve assessment of potential human exposure in their respective study areas and fields of application (i.e. earthquake, tsunami, volcanism). Regarding population distribution modeling for DRM, this thesis is concerned with the total number of people present in a given area. This focus has the following reasons:

a) For many hazard events, especially sudden ones or those above certain magnitude, all human beings are approximately equally vulnerable (Villagrán 2006); 
b) Worldwide and in specific regions, the total population count of a geographical area is still affected by issues and significant uncertainties (see Chapter 5), therefore benefiting from improvements;

c) If the most simple, basic, and neutral demographic variable (total population) is still affected by significant uncertainties, it is likely these are even greater for more complex and sensitive variables (e.g. sex, age, ethnicity) ;

d) Other attributes of population (e.g. sex, age) are often only available at coarser scales (i.e. higher administrative levels) than total population $^{33}$, and disaggregation and gridding of these variables is typically conducted applying the ratio from source zones to all disaggregated total population cells in each zone - therefore propagating the errors in mapping total population. In this scenario, improving grids of total population will also benefit mapping of related demographic attributes.

Each chapter puts these developments in their respective context, justifies why new data is needed and demonstrate its usability and contribution to analysis of human exposure and DRM. In addition, Chapters 2, 3, 4, and 6 show development of improved population distribution grids and their application to specific hazard scenarios, natural and man-made. These population grids were produced using variations of the 'dasymetric mapping' approach, and these layers were previous unavailable for the respective study areas at these spatiotemporal resolutions.

With the exception of Chapter 2, which uses fictitious hazard data, all analyses assess population exposure by relying on best-available hazard maps and data for the respective study areas. Table 3 summarizes the main characteristics of chapters in this thesis in relation to main research topics and concepts.

33 As confirmed in CIESIN GPWv4.11 metadata: https://sedac.ciesin.columbia.edu/downloads/docs/gpw-v4/gpw-v4documentation-rev11.pdf 
Table 3 Main characteristics of chapters in this thesis in relation to main research topics and concepts; main innovative aspects highlighted in bold

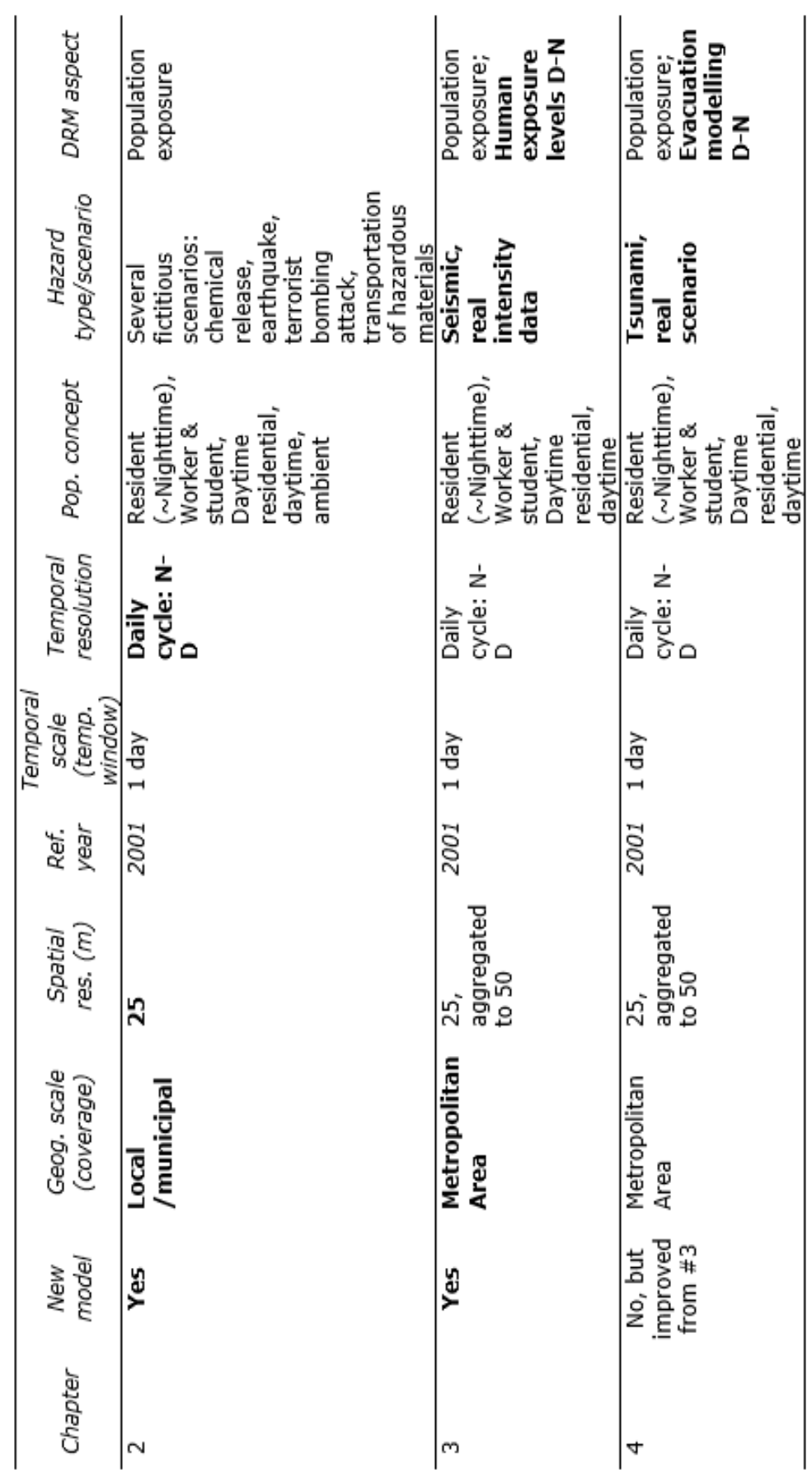


Table 4 (cont.) Main characteristics of chapters in this thesis in relation to main research topics and concepts; main innovative aspects highlighted in bold

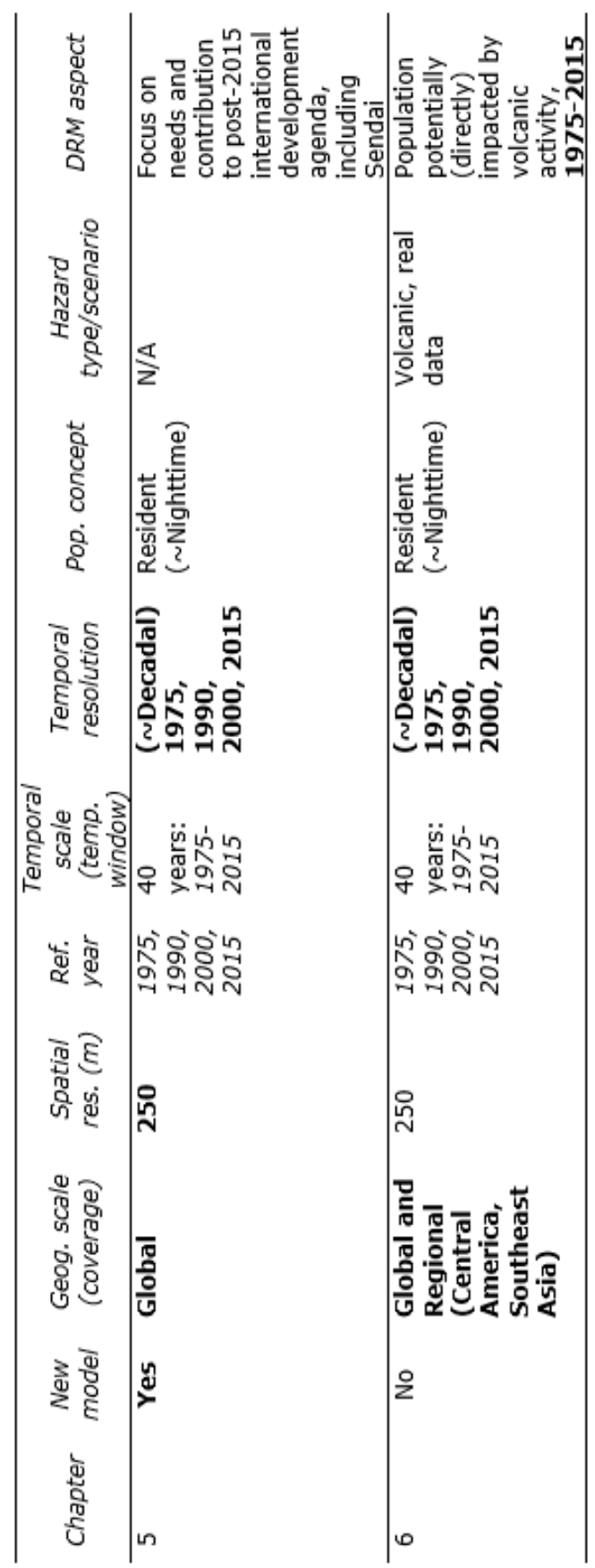




\section{Chapter 2. Modeling of spatio-temporal distribution of urban population at high-resolution - value for risk assessment management ${ }^{34}$ and emergency}

34 This chapter is published as:

Freire, S., 2010. Modeling of spatio-temporal distribution of urban population at high-resolution - value for risk assessment and emergency management. In: Konecny, M., Zlatanova, S., Bandrova, T.L. (eds.), Geographic Information and Cartography for Risk and Crisis Management. Lecture Notes in Geoinformation and Cartography. Springer Berlin Heidelberg, pp. 53-67. 
Modeling of spatio-temporal distribution of urban population at high-resolution - value for risk assessment and emergency management

\begin{abstract}
Knowing the spatiotemporal distribution of population at the local scale is fundamental for many applications, particularly in risk analysis and emergency management. Because of human activities, population counts and their distribution vary widely from nighttime to daytime, especially in metropolitan areas, and may be misrepresented by census data. This study uses a dasymetric mapping approach to refine population distribution in Portugal. The most recent census enumeration figures and mobility statistics are combined with physiographic data to allocate nighttime population to residential areas, and workplaces and workforce are georeferenced to model daytime distribution. Main results represent expected maximum daytime population and maximum nighttime residential population for each $25-\mathrm{m}$ grid cell in the study area. Since the same spatial reference base is used to allocate population, day and night distributions are directly comparable, as is demonstrated in sample applications in the context of emergency management. Verification and validation procedures and demonstrations indicate that the approach suits the objectives.
\end{abstract}

\title{
2.1 Introduction
}

\subsubsection{Population distribution and emergency management}

Natural or man-made disasters, either resulting from natural hazards, technological accidents, or terrorism, usually occur without warning and can potentially affect people from a local to a continental scale. Risk is usually defined as a function of hazard probability and vulnerability, the latter resulting from a combination of exposure and ability to cope (UNDP 2004). Misestimating one of these components necessarily affects the accuracy of the overall risk assessment and mapping.

Human life is unquestionably the most important asset to protect, and the distribution and density of the overall population is a rather basic geographical indicator. Therefore accurately estimating population exposure is recognized as a key component of catastrophe loss modeling, one element of effective risk analysis and emergency management (FEMA 2004, Chen et al. 2004, NRC 2007). However, until recently, assessment and mapping of human vulnerability has been lagging behind hazard analysis efforts (Pelling 2004), and potential loss derived from exposure has served as a quantitative proxy for risk (Lerner-Lam 2007). Also, updated and detailed mapping of population distribution is important for decision support in practically every phase of the emergency management cycle, if produced at appropriate spatial and temporal scales (Sutton et al. 2003), e.g., central to the planning stage is the assessment of population exposure and vulnerability in the hazard zone; mitigation includes reducing vulnerability and exposure (namely by displacing population or other measures); preparedness may involve fitting and placing 
means and resources according to vulnerability, for more efficient response in case of disaster; during and after the event, locating and estimating victims is essential to tailor response and rescue efforts, including allocating emergency personnel, hospital beds, etc.; and finally, estimating all activities and people affected, even if indirectly, facilitates the recovery process.

Despite recent efforts by Dobson (Dobson 2003, 2007) at devising a population estimation technique that could be employed in real time once a disaster occurs, for planning and simulation purposes and to ensure a timely response, adequate population distribution data should be produced and made available beforehand whenever possible.

\subsubsection{Population in space and time}

The increased availability of digital spatial data combined with improved capabilities of Geographic Information Systems (GIS) have allowed for the development of several global population distribution databases, such as the GPW, HYDE, and LandScan (Tobler et al. 1995; Goldewijk and Battjes 1997; Dobson et al. 2000). However, their spatial resolution is still too coarse to adequately support analysis at the local level and most do not represent the dynamics of population distributions. Dobson acknowledges that "even finer resolutions are needed for many types of disasters", namely those that can "impact areas as small as a neighborhood, city block, or single building" (Dobson 2002). Data sets may be produced globally, but people are always affected locally.

Due in part to the complex nature of population as a geographical variable, several approaches have been adopted to estimate their spatial distribution, including statistical modeling (correlation), surface modeling, and cartographic methods (Fisher and Langford 1996; Wu et al. 2005). However, many of these methods require assumptions that over-simplify the reality or disaggregate population totals based on heuristic or empirical parameters. Additionally, obtaining positive values and preserving the total volume of people-Tobler's "pycnophylactic condition" (Tobler 1979)-are basic requirements to produce realistic representations. Dasymetric mapping is a cartographic technique, originally used for population mapping, which aims at limiting the distribution of a variable to the areas where it is present, by using related ancillary information in the process of areal interpolation (Eicher and Brewer 2001).

Population distributions are not static in time, varying over daily, seasonal, and long term time scales (Sutton et al. 2003) due to a number of human activities, such as work and leisure. For emergency planning in urban areas, it is the population variation during the daily cycle that is particularly important to be able to estimate the number, age, and socioeconomic classes affected by an impact (Alçada-Almeida 2009). In Portugal, as in most countries, existing population distribution maps and vulnerability or exposure analyses are 
Modeling of spatio-temporal distribution of urban population at high-resolution - value for risk assessment and emergency management

normally based on census data, often aggregated at the commune level (e.g., Oliveira et al. 2005). Census figures register where people reside and usually sleep, although their spatial distribution varies widely between night and day, especially within metropolitan areas. Due to daily commuting alone, the daytime population of municipalities in the metro areas of Lisbon and Porto can differ by more than $50 \%$ of the official census figures (INE 2003). Also, the availability of a total count for census or administrative areas creates problems for analysis (e.g., MAUP, ecological fallacy), being generally assumed that the distribution is constant and exhaustive in those areas, or represented by their centroid.

Making population distribution data available as a high-resolution raster database facilitates rapid GIS analysis at the local level and for any zoning. Therefore, when disaster strikes or is imminent, knowing how many people are likely to be in the affected area can be invaluable information for adequate emergency response and evacuation planning. The LandScan project (Dobson et al. 2000) successfully incorporated the temporal dimension in population distribution by mapping "ambient population", a temporally averaged measure of population density that accounts for human activities (such as sleep, work, study, transportation, etc.). This representation may be more adequate for certain applications (such as emergency management) than residence-based population density. However, LandScan's spatial resolution (30 arc-seconds), while adequate for national to global analyses, is insufficient for most practical uses in Portugal. Furthermore, ambient population corresponds to a compromise between daytime and nighttime distributions that strictly represents neither period. Therefore, population distribution databases having higher temporal and spatial detail are being developed for the territory of the USA (McPherson and Brown 2004, Bhaduri et al. 2002).

This study is aimed at developing and testing a data-based model to map the nighttime and daytime population distributions in Portugal at high resolution to enable local-level analysis. This effort correlates with recent recommendations to improve vulnerability analyses (Cutter 2003, Balk et al. 2006, Birkmann 2007).

\subsection{Methods}

Pre-processing and modeling of geographical data were conducted in ESRI $\mathbb{R}$ ArcGIS 9.1, a GIS application. GIS offers the necessary tools and flexibility to implement raster or vector-based dasymetric methods, and was used to verify, correct, and integrate geographic data sets, and for modeling, analysis, and mapping the results for presentation.

Because some data sets were made available on a municipal basis, each municipality was modeled separately. For each municipality, 25-m raster grids were created representing different types of population distribution. The raster 
structure provides uniform and flexible units that facilitate the reaggregation for any zoning, thus being suitable for modeling and analysis. A spatial resolution of $25 \mathrm{~m}$ was adopted to approximate the size of a single-family residence (half block). Also, when the model was tested for sensitivity to cell size, an increase in resolution to $12.5 \mathrm{~m}$ yielded marginal gains in the accuracy of results.

\subsubsection{Study area}

The official administrative limits of the municipalities (concelhos) of Cascais and Oeiras in 2001 constitute the study area for this research. These are two of the 18 municipalities that comprise the Lisbon Metropolitan Area (LMA), the main metropolitan area in Portugal (Figure 3).

Cascais and Oeiras occupy 97 and 46 km2, respectively, and have a combined resident population of 332,811 ; this results in an average population density of 2,332 inhabitants/ $\mathrm{km} 2$, well above the national average density of 112 inhabitants $/ \mathrm{km} 2$. However, population density varies widely throughout the study area, from high density in multistory residential apartments to low density in rural areas. Even at the census block group level, some polygons are quite large and do not reflect their uneven population density.

This area was selected for several reasons: (a) its characteristics, namely with regard to urban and suburban character, and strong economic activity, (b) the availability and access to input data, and (c) personal familiarity with the area, which facilitates data verification and field work. 


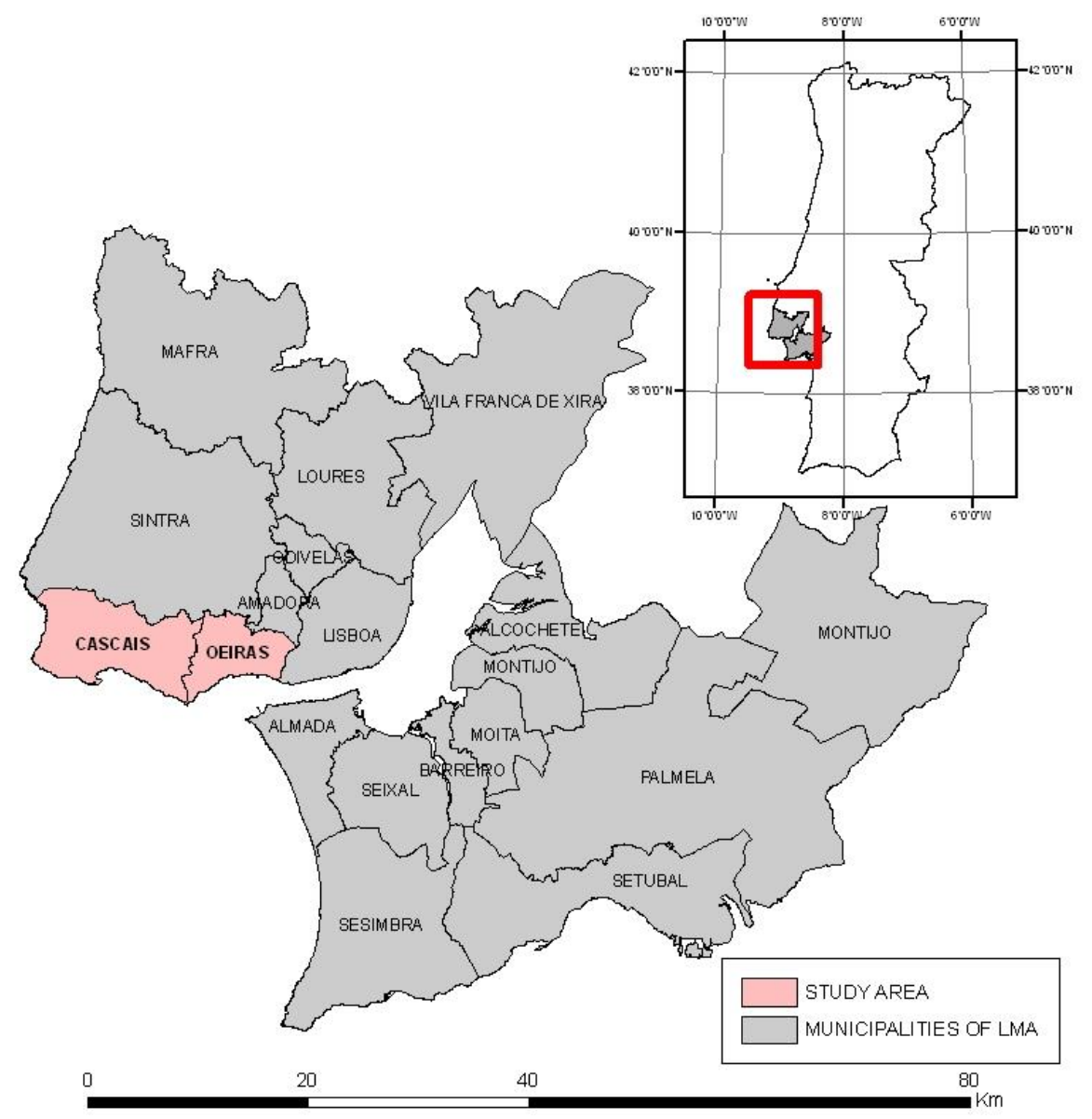

Figure 3 Location of the study area in Portugal and in the Lisbon Metro Area (LMA) (Source: CAOP).

\subsubsection{Data sets}

In this study, the spatial detail of census zones whose counts are to be disaggregated should be met in scale, resolution, and accuracy by ancillary data sets used for disaggregation. Input variables used for modeling include both physiographic and statistical data. In the first group are census tracts, street centerlines, and land use and land cover (LULC), while the second includes census counts (INE 2001), data on workforce by workplaces, and commuting statistics (INE 2003) for the study area. These data were obtained from various sources and in different formats which are listed in Table 5. 
Table 5 Main input datasets used for modeling nighttime and daytime population

\begin{tabular}{|c|c|c|c|}
\hline Data set & Source & Date & Data type \\
\hline Street centerlines & Private & 2004 & Vector polyline \\
\hline LULC (COS90; CLC2000) & Public & $1990 ; 2000$ & Vector polygon \\
\hline Census block groups & Public & 2001 & Vector polygon \\
\hline Census statistics & Public & 2001 & $\begin{array}{l}\text { Database } \\
\text { Access) }\end{array}$ \\
\hline $\begin{array}{l}\text { Workplaces } \\
\text { employment }\end{array}$ & Public & 2001 & Table \\
\hline Commuting statistics & Public & 2001 & Table (O/D matrix) \\
\hline
\end{tabular}

In general, temporal consistency among data sets was very high, with the exception of street centerlines whose reference date was 3 years subsequent to the model target date (2001). For this reason and owing to the importance of this data set in the model, it was decided to modify it in order to better represent the reality of the target date. The LULC data originated from two maps and was also corrected and improved upon within the study area.

\subsubsection{Model}

The modeling of population distribution is based on dasymetric mapping using street centerlines as spatial reference unit to allocate population counts. The most recent statistical and census data (2001) provide the population counts for each daily period, while physiographic data sets define the spatial units (i.e., grid cells) used to disaggregate those counts. This general approach was successfully implemented by the Los Alamos National Laboratory (New Mexico, USA) to map daytime and nighttime population distributions in the United States at 250-m resolution (McPherson and Brown 2004), and is adapted and applied to Portugal.

An overview of tasks involved in this modeling process is presented in Figure 4. Input data are noted in light gray, secondary products previously unavailable in Portugal are noted in bold, and main results are noted in bold and darker gray. 
Modeling of spatio-temporal distribution of urban population at high-resolution - value for risk assessment and emergency management

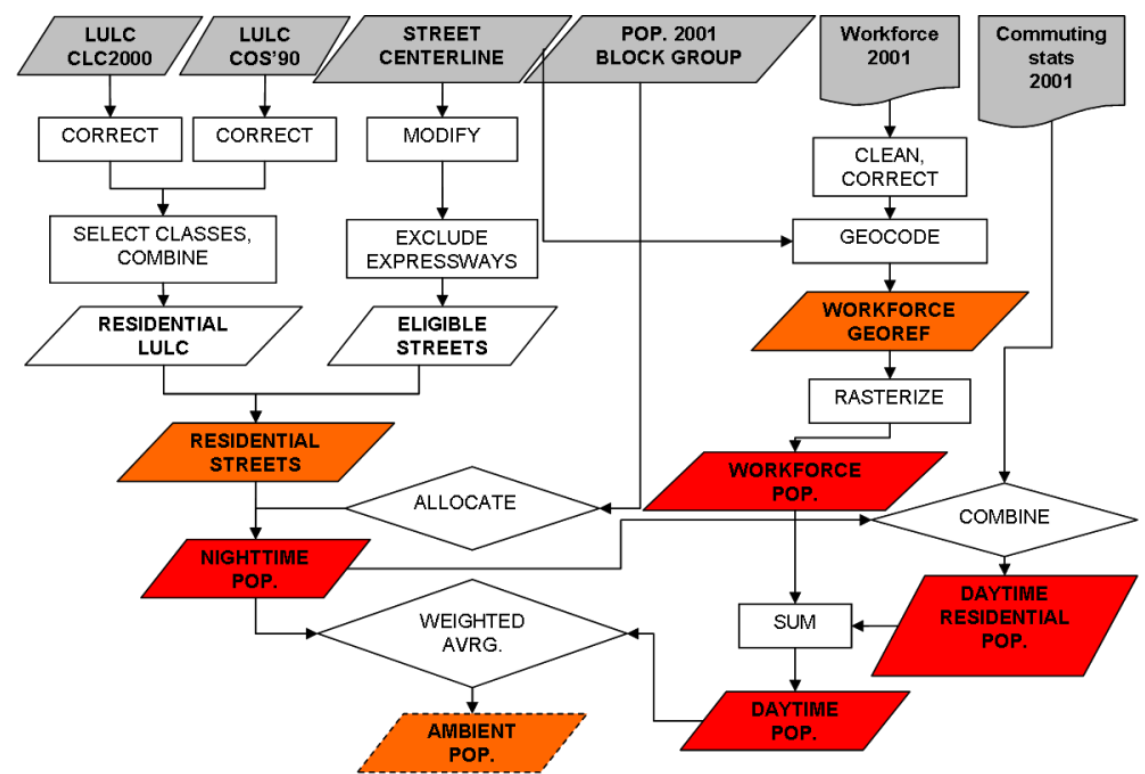

Figure 4 Flowchart of main tasks involved in the model: input data are noted in light gray, secondary products in orange, and main results noted in bold and red

The map of nighttime population distribution was obtained by using a grid binary dasymetric mapping method to disaggregate residential population from census zones to residential streets. First, available digital LULC maps were improved, relevant classes selected, and combined, in order to identify residential land use. Street centerlines were also modified in order to better represent the road network existing in 2001. Then, freeways are removed from consideration and the resulting eligible streets are combined with residential land use classes from LULC data to obtain residential streets. These are subsequently rasterized at $25 \mathrm{~m}$ and the population from census block groups (source zones) are interpolated to the respective residential street cells (target zones) using areal weighting.

The daytime population distribution results from the combination of two components (as illustrated in Figure 4): (a) the daytime population in their places of work or study-the workforce population surface, and (b) the population that remains home during the day-the daytime residential population grid. The latter is obtained by multiplying the nighttime distribution by the percentage of resident population who, according to official statistics (INE 2003), do not commute to work or school. In the absence of other information, it is assumed that non-commuters remain in their residences in the daytime period. This implies that this study is not including the potential effects of 
leaving home for shopping and several other activities on daytime population distributions.

The workforce population surface was created by georeferencing 4,316 workplaces and schools and respective workforce and students in the study area; 1,395 of these were georeferenced manually using ancillary data and field work. The remainder workplaces were geocoded to the street centerlines in ArcGIS using their addresses. Total daytime population surface results from the sum of workforce population with daytime residential population on a cellby-cell basis. The ambient population distribution is estimated by computing a weighted average of nighttime and daytime distributions, considering the proportion of nighttime and daytime periods occurring in a typical 7-day weekly cycle.

\subsection{Results}

Main results consist of raster surfaces of nighttime (residential) population, daytime residential population, daytime worker and student population, total daytime population, and ambient population. These have the number of people in each $25-\mathrm{m}$ cell in 2001, thus representing population density by $625 \mathrm{~m} 2$. Nighttime and daytime distributions represent maximum expected density on a typical workday, assuming that everyone is at home at night and all workers and students are in their workplaces and schools, and the remainder in their residences. Although still a simplification of reality, it is preferable than computing a zonal average by census polygon alone.

\subsubsection{Verification and validation}

Validation of daytime population distribution was limited by unavailability of compatible reference data sets for Portugal. However, this limitation can be considered less relevant since daytime population distribution originates from a combination of the nighttime distribution surface (subject to formal validation) with mostly official statistical data, as opposed to being derived from heuristic or empirical weights. Still, the daytime population distribution was subject to verification in several ways: (a) input data (especially workplaces' addresses) were verified through cross-checking with other sources and field work, regarding location of workplaces (firms), (b) results were verified with high-resolution imagery to confirm positional accuracy of distributions, and (c) it was checked that the total number of workers provided and other statistics (census and mobility) were not contradictory.

The nighttime population distribution was subject to a formal accuracy assessment process, using the higher-resolution census blocks as reference (i.e., ground truth) in a correlation analysis. Cell values in modeled distributions were aggregated by census block in ArcGIS and compared against the respective census count. Correlation coefficients (Pearson's r) of 0.84 were 
Modeling of spatio-temporal distribution of urban population at high-resolution - value for risk assessment and emergency management

obtained for the municipality of Cascais and 0.79 for the municipality of Oeiras. Even when the 36 blocks that coincide with block groups are not considered, the coefficients decrease only slightly to 0.79 and 0.75 , respectively. This indicates that model performance is rather good, in light of its high resolution and considering the large number of samples used for validation $(2,433$ census blocks in Cascais, 1,456 in Oeiras).

\subsection{Sample applications}

Three fictitious case study scenarios are presented as sample applications of model results in the context of risk analysis and emergency management. Situations are examples of technological hazards, natural disasters, and terrorist attack. These scenarios illustrate the usefulness of improved population distribution mapping for planning or response to actual events.

\subsubsection{Case study A: technological hazard (airborne toxic plume release)}

A truck transporting highly toxic chemical products is involved in a serious accident on the off-ramp of a busy freeway in Oeiras, at 11:00 hours (AM). An airborne toxic plume is released and the dominant winds slowly push it southwards. Figure 5 represents this scenario, showing the plume and the daytime population distribution overlaid on orthophotos from 2004. 


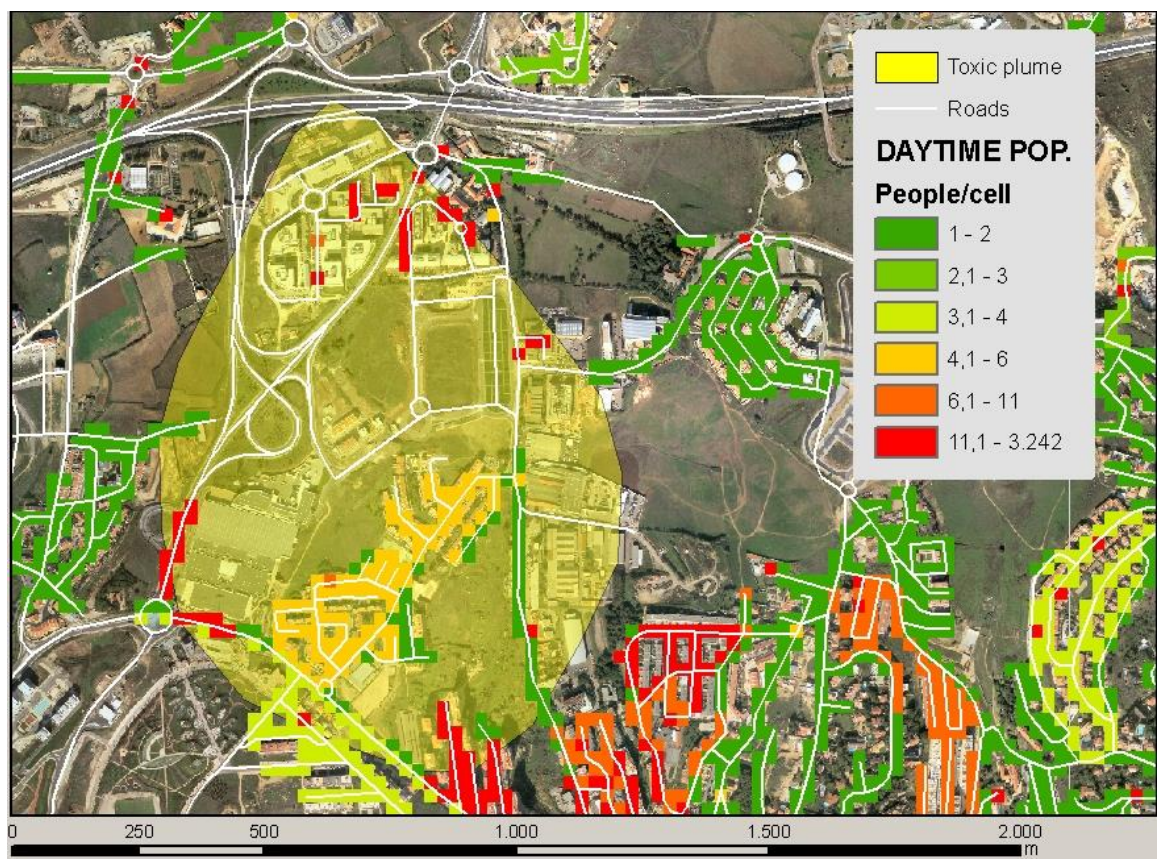

Figure 5 Case study A: airborne toxic plume release in Oeiras (Source: IGP; TeleAtlas).

\subsubsection{Case study B: natural disaster (earthquake)}

The Lisbon Metro Area is located in a high seismic risk area, having suffered destructive earthquakes in the past (e.g., 1755). Around 15:00 hours a strong earthquake strikes the town of Cascais, causing major damage around downtown and along the coast. Figure 6 represents this scenario, showing the area most seriously affected by destruction and collapse of buildings, and the daytime population distribution overlaid on 2004 orthophotos. 
Modeling of spatio-temporal distribution of urban population at high-resolution - value for risk assessment and emergency management

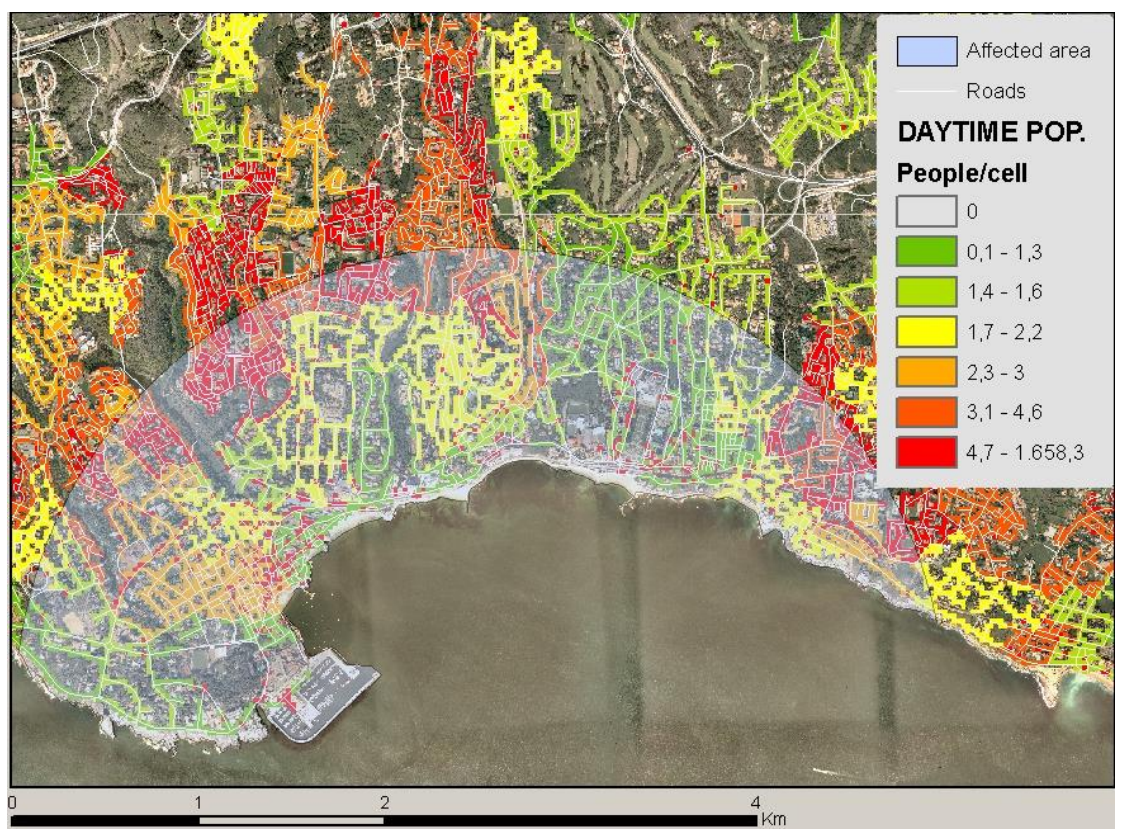

Figure 6 Case study B: earthquake affecting downtown Cascais (Source: IGP; TeleAtlas).

\subsubsection{Case study C: terrorist attack (bombing of shopping center)}

Around 16:30 hours a powerful explosive is detonated in a large shopping mall in Cascais, causing great destruction and a fire. Figure 7 represents this scenario, showing the area most affected by the blast, and the daytime population distribution overlaid on 2004 orthophotos.

Based on the scenarios presented above, it is relatively straightforward to use GIS analysis to calculate the affected (exposed) population in each event. Table 6 quantifies and compares exposure based on direct use of census data versus use of appropriate model results. In all the scenarios there are important differences in the figures of total exposed population using census data compared to more appropriate model data. In these daytime scenarios, use of census data indicates significant under-estimation of affected population, more dramatic in Scenario C. This could possibly lead the authorities to underprepare the emergency response, resulting in an increase of more serious victims or unnecessary suffering. Also, use of daytime distributions to simulate and plan for daytime events also improves assessment of exposure and may contribute to better planning and mitigation measures. 


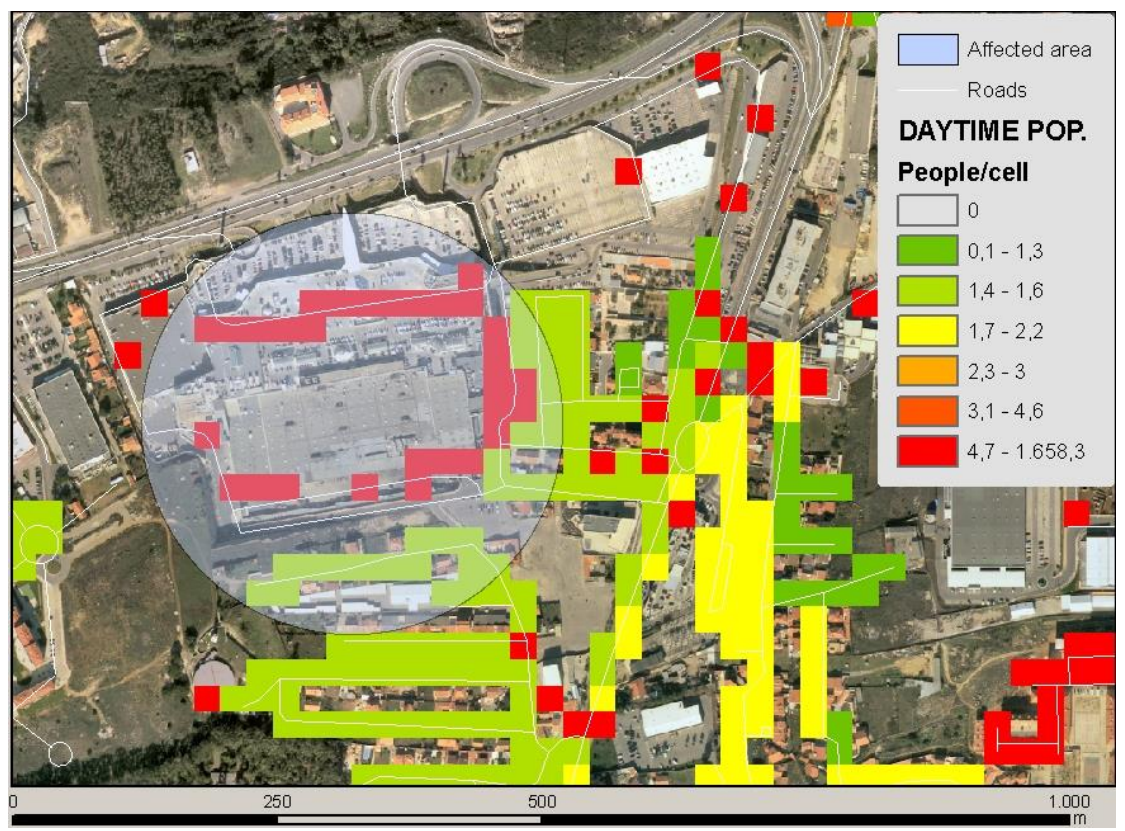

Figure 7 Case study C: terrorist attack in a Cascais shopping center (Source: IGP; TeleAtlas).

Table 6 Total exposed population in each case study

\begin{tabular}{llll}
\hline Scenarios & $\begin{array}{l}\text { Census data } \\
\text { (Block group) }\end{array}$ & $\begin{array}{l}\text { Model results } \\
\text { (Daytime pop.) }\end{array}$ & $\begin{array}{l}\text { Difference } \\
\text { [persons (\%)] }\end{array}$ \\
\hline A & 2,144 & 3,950 & $1,806(84 \%)$ \\
B & 22,841 & 28,944 & $6,113(26 \%)$ \\
C & 86 & 2,151 & $2,065(2,400 \%)$ \\
\hline
\end{tabular}

\subsubsection{Case study D: planning of best route for hazardous materials transportation}

Scenario D illustrates the use of spatiotemporal population distribution in a planning stage as a criterion for truck routing of hazardous materials between the A5 freeway exit and a processing facility, in an industrial area of the municipality of Oeiras. Consideration of population distribution in this context allows two fundamental issues to be addressed simultaneously: what is the best period (day or night) and route in order to minimize population exposure along the way?

In the example, population distribution in a $50-\mathrm{m}$ wide buffer along the streets is considered as the cost impedance to select the route between two locations which minimizes overall population exposure. Best routes were computed for the nighttime period and for the daytime period, considering each period's 
Modeling of spatio-temporal distribution of urban population at high-resolution - value for risk assessment and emergency management

respective population distribution resulting from the model as the sole criterion (Figure 8).

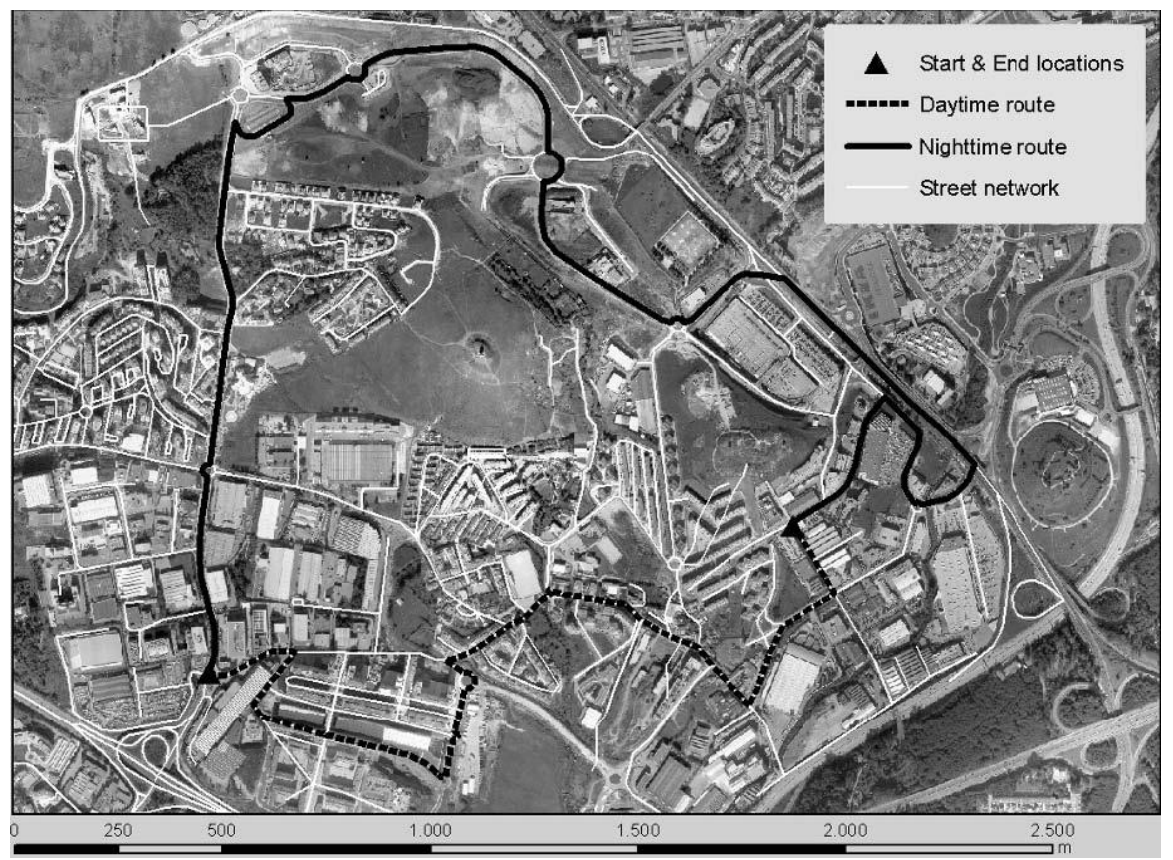

Figure 8 Case study D: best route considering the population distribution (Source: IGP; TeleAtlas).

Table 7 quantifies and compares overall population exposure along each route and in each period.

Table 7 Total exposed population along each route and period

\begin{tabular}{lllll}
\hline \multirow{2}{*}{ Best routes } & \multicolumn{4}{l}{ Exposed population } \\
\cline { 2 - 5 } & $\begin{array}{l}\text { Length } \\
{[\mathrm{km}]}\end{array}$ & Nighttime & Daytime & $\begin{array}{l}\text { Difference } \\
\text { [persons }(\%)]\end{array}$ \\
\hline Nighttime & 4,9 & 345 & 3352 & $3007(872 \%)$ \\
Daytime & 2,7 & 1001 & 2734 & $1733(173 \%)$ \\
\hline
\end{tabular}

Results show that consideration of spatiotemporal population distribution for routing leads to definition of quite different routes, having quite different lengths. Although the best route for the nighttime period is $4.9 \mathrm{~km}$ long, only 345 people are within the affected zone, whereas in the daytime period 2,734 people would be exposed along a shorter $2.7-\mathrm{km}$ route. Additionally, total exposure along the best route for the nighttime period varies widely between night and day, increasing by 3,007 people to surpass the daytime's best route exposure. Therefore, in order to minimize overall population exposure it is the 
nighttime period and longest route that should be selected for truck routing of hazardous materials in the study area.

\subsection{Conclusions}

As the population is not static, it was demonstrated that emergency management activities would greatly benefit from the use of reliable population distribution data with increased spatiotemporal resolution for estimation of human exposure and vulnerability. GIS enables both the development and spatial analysis' applications of these data sets. The adopted approach, based on official data, allows the mapping of nighttime and daytime population distribution at high spatial resolution, to support local-level analysis. This method also efficiently accommodates people that work at home, by not considering that all active population leaves their residences during the workday. The main value of these results includes the increased spatial resolution of nighttime distribution, the fact that both nighttime and daytime distributions share the same spatial reference basis, and that daytime distribution is better approximated. Furthermore, the combination of both distributions yields an approximate representation of ambient population. The dasymetric mapping technique and zonal interpolation meet the requirements for disaggregation of population counts-obtaining positive values and preservation of total mass-so the final distribution matches official statistics and counts.

Given the availability of input data sets, this approach could be applied to all municipalities (19) comprising the Metropolitan Areas of Lisbon and Porto, that together contain $40 \%$ of Portugal's population. Subsequent versions would benefit from a number of improvements: better modeling of "distributed activities" (e.g., cleaning, security), and accounting for people present in transportation networks, in hospitals and prisons, or involved in leisure and shopping activities; increased temporal segmentations of population distribution, so as to represent differences on a weekly basis (workdays vs. weekend) or on a seasonal basis (winter vs. summer); and the use of statistical sources beyond census demographics to consider tourism influx in areas and periods where that activity is significant. In the context of exposure and risk analysis, it would also be useful to model people who are indoors versus those involved in outdoor activities.

\section{Acknowledgement:}

The kind support of GeoPoint Lda. throughout this project was greatly appreciated. The author also thanks the Lisbon Metropolitan Area for providing data on school facilities, from the Sistema Metropolitano de Informação Geográfica - Grande Área Metropolitana de Lisboa 
Modeling of spatio-temporal distribution of urban population at high-resolution - value for risk assessment and emergency management 


\section{Chapter 3. Integrating population dynamics into mapping human exposure to seismic hazard ${ }^{35}$}

35 This chapter is published as:

Freire, S., C. Aubrecht, 2012. Integrating population dynamics into mapping human exposure to seismic hazard. Natural Hazards \& Earth Systems Sciences, 12(11):3533-3543. 


\begin{abstract}
Disaster risk is not fully characterized without taking into account vulnerability and population exposure. Assessment of earthquake risk in urban areas would benefit from considering the variation of population distribution at more detailed spatial and temporal scales, and from a more explicit integration of this improved demographic data with existing seismic hazard maps. In the present work, "intelligent" dasymetric mapping is used to model population dynamics at high spatial resolution in order to benefit the analysis of spatiotemporal exposure to earthquake hazard in a metropolitan area. These nightand daytime-specific population densities are then classified and combined with seismic intensity levels to derive new spatially-explicit four-class composite maps of human exposure. The presented approach enables a more thorough assessment of population exposure to earthquake hazard. Results show that there are significantly more people potentially at risk in the daytime period, demonstrating the shifting nature of population exposure in the daily cycle and the need to move beyond conventional residence-based demographic data sources to improve risk analyses. The proposed fine-scale maps of human exposure to seismic intensity are mainly aimed at benefiting visualization and communication of earthquake risk, but can be valuable in all phases of the disaster management process where knowledge of population densities is relevant for decision-making.
\end{abstract}

\title{
3.1 Introduction
}

\subsubsection{The importance of population dynamics for disaster risk assessment}

Even in the context of natural hazards, risk has several definitions and multiple approaches exist for its assessment and mapping (Adger, 2006; Birkmann, 2006; Villagran, 2006). The United Nations, for example, define disaster risk as a function of hazard probability and vulnerability, the latter resulting from a combination of exposure and ability to cope (UNDP, 2004; UNISDR, 2009). Among the different types of elements that may be present in hazard zones (people, property, systems, etc.), human life is unquestionably the most important value to protect from disasters. The elements of vulnerability and population exposure are present in some form in all the conceptualizations of risk and natural disasters, and risk is not fully characterized without an assessment of those components, in addition to the hazard itself. However, assessment and mapping of social vulnerability has traditionally been overlooked in favor of hazard modeling studies (Pelling, 2004; Douglas, 2007). The development of sophisticated and detailed numerical-based modeling of hazard zones (e.g. seismic intensity zones, tsunami flood depth, chemical dispersion models) contrasts with the use of static, generalized, residencebased representations of population exposure using census data. Nonetheless, 
vulnerability is regarded as a greater contributing factor to disaster risk than the mere existence of hazards (Uitto, 1998; Alexander, 2006; EEA, 2010).

Population exposure refers to the human occupancy of hazard zones (Cutter, 1996), or the population present within the hazard area that would be potentially directly affected by an event. For many hazard occurrences, especially those above a certain magnitude or intensity, population exposure is arguably the greatest determinant of vulnerability and resulting losses and impacts. Therefore, it has progressively been acknowledged that the accurate estimation of population exposure as a vital component of catastrophe impact modeling is an essential element of effective risk analysis and emergency management (Chen et al., 2004; FEMA, 2004). Despite the study of exposure and vulnerability to hazards being increasingly at the core of hazards and disaster research, the inclusion of socioeconomic variables into geospatial risk models implemented within a Geographic Information System (GIS) remains a challenge (EC, 2010). Quantifying population exposure as a step for conducting spatially-explicit risk assessment requires mapping the spatial distribution of population with sufficient resolution. Population data is therefore a basic necessity for human exposure analysis, with its quality and level of detail having a direct effect on response and lives saved (NRC, 2007). Updated and detailed mapping of population distribution at appropriate spatial and temporal scales provides an important basis for decision support in every phase of the emergency management cycle (Sutton et al., 2003; Freire, 2010; Aubrecht et al., 2012a). Concerning the spatial dimension, disaster risk reduction and mitigation demand measures implemented at local level, which requires understanding of vulnerabilities at compatible scales (Lerner-Lam, 2007). Since natural hazards can affect urban areas in a very selective manner, only fine-scale population data can provide an accurate estimate of the population affected (Deichmann et al., 2011) Analysis of pre-event population distribution is necessary for establishing a base-line situation for assessing risk, and pre-event maps are often needed during the response phase (Zerger and Smith, 2003). Also, due to limited real-time capability for mapping population distribution, such data sets should be prepared ahead of time, despite efforts by Dobson (2007) at developing a bottom-up population estimation technique, based on Building Occupance Tables, which could be employed once a disaster occurs.

Increasing population density and mobility has been contributing to growing vulnerability of social systems (EEA, 2010). Due to the diverse locations of human activities and the displacements they induce, the spatial distribution of population is strongly time-dependent, especially in metropolitan areas. For the temporal shifting of population exposure, the most important determination is whether an incident occurs at night or during the day (Dobson, 2007). However, temporal variations of risk, due to changes in the human component involving population and additional socioeconomic assets, are still 
rarely included in pre-event assessments conducted by emergency managers (Kakhandiki and Shah, 1998). This striking fact may be due to both lack of appropriate data during the planning stage, and failure to perceive the dynamics of risks. In any case it contrasts sharply with decision makers' shift to focus on temporal detail once a disaster strikes (Zerger and Smith, 2003; Goodchild, 2006). Therefore, Geographic Information Science research needs to include improved integration of physical processes and socioeconomic models in disasters and emergency management. Required improvements also include visual depictions of risks and vulnerability that represent their spatial and temporal shifts at local level (Cutter, 2003; Aubrecht et al., 2012b). For improved analysis of human exposure in large urban areas and to facilitate integration with hazard zones, population distribution data should be available as high-resolution raster data sets depicting at least a day-night estimation of its variation (Freire, 2010).

Motivated by concerns with homeland security and emergency management, two such nighttime and daytime population distribution databases were developed in the last decade for the US: LandScan USA, having a 90-m ( 3 arcs) cell size (Bhaduri et al., 2002; Dobson et al., 2003), and 250-m resolution day and nighttime grids produced by the Los Alamos National Laboratory (McPherson and Brown, 2004).

\subsubsection{Population exposure to seismic hazard}

For efficient and effective risk management, hazard and vulnerabilities should be assessed before a disaster strikes (Birkmann, 2007), which requires the creation and maintenance of baseline data as part of geospatial preparedness activities (Emrich et al., 2011). This is especially relevant in the case of earthquakes, whose damaging effects are compounded by the impossibility of accurate and timely forecasting (Geller, 1997; Buchanan, 2001; Guo, 2010). Earthquakes are rapid-onset, short-duration, time-specific and potentially high-consequence events, having long been the prototype for a major disaster. They have local to regional geographical impact (Peduzzi et al. 2009b), often causing significant secondary hazards and cascading impacts such as fire, flooding, and release of hazardous chemicals (Rashed and Weeks, 2003). Population density, together with building type and event magnitude, is one of the main factors determining damage from an earthquake (Ambraseys and Jackson, 1981). In an assessment of health effects of past earthquakes, Alexander (1996) notes that the risk of injury varies significantly between night and day, which leads to the recommendation that vulnerability and exposure should be assessed in this temporal cycle.

Contrary to other hazards (e.g. forest fires), earthquake risk cannot be addressed by taking actions that lower the hazard component (i.e. location, geographic scope, frequency, duration, and magnitude); instead, only by 
decreasing the vulnerability (of structures and people) can this risk be mitigated. For implementing those measures, modeling, mapping, and quantifying population exposure forms an essential first step. On a local scale, Taubenbock et al. (2008) have illustrated for a single district in Istanbul, Turkey, the importance of considering population dynamics for assessing the spatial distribution of risks in case of earthquake. Aubrecht et al. (2011) have demonstrated how high-resolution population data, disaggregated to building level, can improve pre-event estimation of human exposure to potential earthquake hazard in an urban area. On a global scale, the USGS' PAGER (Prompt Assessment of Global Earthquakes for Response) system estimates after an event the number of people exposed to shaking by using the coarse population distribution surface from LandScan (Dobson et al., 2000). However, most studies on population exposure to earthquake hazard only consider census-based resident population (i.e. nighttime) and often fail to integrate population distribution data with actual seismic hazard maps. Therefore, there is an essential need to advance current state-of-the-art exposure assessment by: (i) accounting for spatiotemporal variation of population distribution in urban areas, and (ii) combining more explicitly and in more detail the best demographic data on the potentially affected population with existing seismic hazard maps.

The main objectives of the present work are to improve earthquake risk analysis at regional level to benefit decision support for disaster and emergency management by (1) modeling and mapping nighttime and daytime population distribution at high spatial resolution, (2) assessing spatio-temporal population exposure to earthquake hazard and (3) classifying exposure levels through the combination of population densities with seismic hazard to derive and propose new fine-scale composite human exposure maps. The approach is presented using the Lisbon Metropolitan Area as the test site.

\subsection{Study area and data}

\subsubsection{Study area}

The test site for this study encompasses the eighteen municipalities that currently compose the Lisbon Metropolitan Area (LMA), the main metropolitan area in Portugal (Figure 9). 


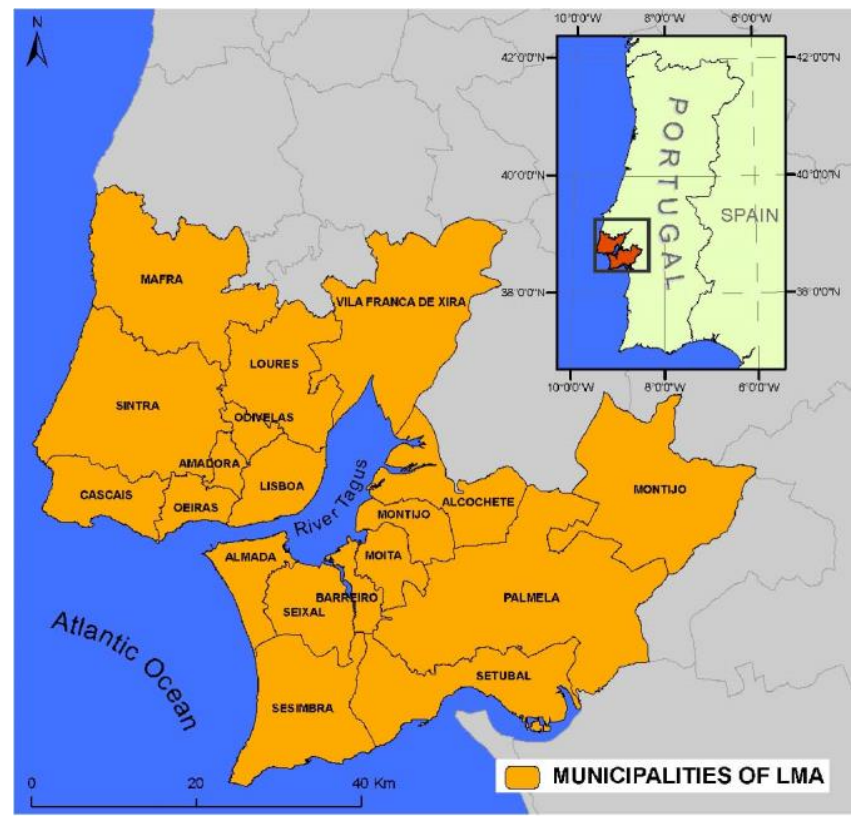

Figure 9 Study area - Lisbon Metropolitan Area (LMA)

This region is characterized by a moderate seismicity with a diffuse pattern, having been affected by historical earthquakes that caused many victims, severe damages and economic losses (Carvalho et al., 2006). These impacts are the outcome of seismic activity occurring in the collision of the Iberian and African plates, resulting in a historical seismicity which includes events originating both in the interplate region (distant source) and in the nearby faults of the intraplate region, including the Lower Tagus Valley. Moderate to large earthquakes originating in this area include those in 1344, 1531 ( $\mathrm{M}=$ $7.2)$, and 1909 ( $M=6.3$ ) (Oliveira, 2008). The famous 1755 event ( $M=8.5-$ 9.0 ), regarded as probably the greatest seismic disaster to have affected Western Europe, occurred around 09:40 a.m., when many people were not in their residences, causing between 60,000 and 100,000 casualties and much destruction (Chester, 2001). A 1755-type event, seen as worst-case scenario for the LMA region, is estimated to have a return period of between 3000 and $4000 \mathrm{yr}$. In the Lower Tagus Valley, earthquake return periods vary between less than $100 \mathrm{yr}$ for $M=5$ to about $1000 \mathrm{yr}$ for $M=7$. Estimation of vulnerabilities is still one of the main uncertainties for earthquake scenarios in Lisbon, and improved inventory of population in the daily cycle is required (Oliveira, 2008).

A "Special Emergency and Civil Protection Plan for Seismic Risk" (PEERS-AML$\mathrm{CL}$ ), approved in 2009, was produced for the LMA and adjacent municipalities (26 in total). The Plan, based on a seismic intensity map, was devised as an operational instrument for organizing response to an event, and is 
automatically activated for an earthquake having a magnitude equal to or greater than 6.1 (Richter) or intensity level VIII (Modified Mercalli). However, the Plan only considers census' resident population in vector format for the assessment of human exposure, therefore merely approximating affected population for a nighttime event.

The LMA accounts for $36 \%$ of the country's GDP and $30 \%$ of all national companies are located there. The 18 municipalities of Lisbon Metro occupy a total land area of 2,963 km2 (3.3\% of Portugal) and are home to 2661850 residents, $26 \%$ of the country's population (INE, 2001). The total resident population has increased $5.6 \%$ from 1991 to 2001 . Although the average population density is 898 inhabitants per square kilometer, these densities vary widely in space and time. Beyond the more urbanized core, the region still includes numerous rural areas with scattered settlements whose uneven population density is not adequately captured and represented by heterogeneous census polygons, which can be quite large even at the block level. Also, due to concentration of activities and daily commuting for work and study, the daytime distribution of the population in the municipalities of the LMA is significantly different from the nighttime period, and their totals can vary by more than $50 \%$ compared to the residential figures from the census (INE, 2003; Table 8).

Table 8 Nighttime and daytime population in the municipalities of the Lisbon Metropolitan Area, in 2001 (derived from INE, 2001, 2003)

\begin{tabular}{lrcr}
\hline Municipality & Nighttime & Daytime & Difference (\%) \\
\hline Alcochete & 13010 & 11374 & -12.6 \\
Almada & 160825 & 146987 & -8.6 \\
Amadora & 175872 & 141253 & -19.7 \\
Barreiro & 79012 & 68193 & -13.7 \\
Cascais & 170683 & 151115 & -11.5 \\
Lisboa & 564657 & 898840 & 59.2 \\
Loures & 199059 & 167315 & -15.9 \\
Mafra & 54358 & 49862 & -8.3 \\
Moita & 67449 & 51895 & -23.1 \\
Montijo & 39168 & 38435 & -1.9 \\
Odivelas & 133847 & 96653 & -27.8 \\
Oeiras & 162128 & 148937 & -8.1 \\
Palmela & 53353 & 54286 & 1.7 \\
Seixal & 150271 & 115779 & -23.0 \\
Sesimbra & 37567 & 32921 & -12.4 \\
Setubal & 113934 & 115854 & 1.7 \\
Sintra & 363749 & 291421 & -19.9 \\
Vila Franca de & 122908 & 103719 & -15.6 \\
Xira & & & \\
\hline Total & 2661850 & 2684839 & 0.9
\end{tabular}

The characteristics of the area and the availability of a recent Seismic Hazard 
Intensity map, in the context of the above-mentioned Special Emergency and Civil Protection Plan, provide an appropriate context for the effort presented in this paper, i.e. contributing to improved risk assessment for that particular hazard type.

\subsubsection{Data sets}

The main data sets produced and used in the course of the presented analyses were population distribution surfaces and a seismic intensity map, respectively (Figure 10).

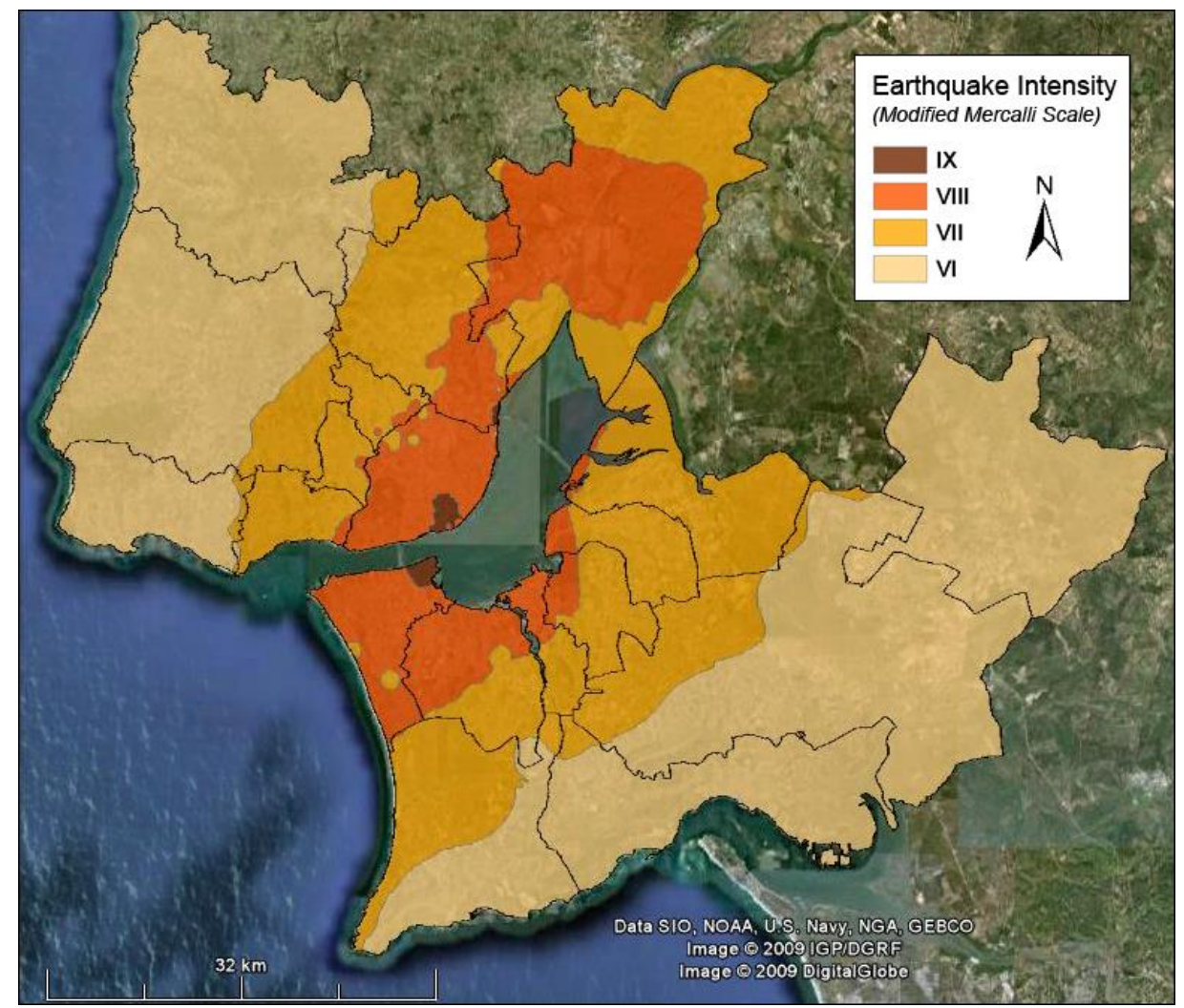

Figure 10 Seismic Intensity map for the study area (background from Google Earth).

Input variables used for modeling population distribution include both physiographic and statistical data. The first group comprises street centerlines and land use/land cover (LULC) maps, while the second includes census counts (INE, 2001), data on workforce, and commuting statistics (INE, 2003) for the study area. These data were obtained from various sources and in different formats, as listed in Table 9. COS90 is a digital LULC map at the scale 1:25 000 , covering almost the entire country using a very detailed legend, however 
it dates from 1990. CORINE Land Cover 2000 (CLC2000) was a pan-European project mapping LULC at the scale 1:100 000 using a hierarchical nomenclature with 44 classes in the most detailed level (level 3). Therefore, to ensure temporal consistency among input data sets, it was decided to update the COS90 base by adding new Artificial Surfaces from the more recent CORINE Land Cover database for the year 2000. That way, high temporal uniformity of the input data set is achieved, which is usually a challenge in geographic modeling. The seismic intensity map was produced for the above-mentioned "Special Emergency and Civil Protection Plan for Seismic Risk" (PEERS-AML$\mathrm{CL}$ ) and represents maximum Seismic Intensity (Modified Mercalli scale) for the area based on the following situation: earthquake events of $M=6.6 / 6.7$ with epicenter in the lower valley of the Tagus river. Based on this scenario, Seismic Intensity in the LMA is expected to vary between levels VI and IX (Figure 10).

\subsection{Methodology}

All processing and modeling of spatial and spatially-related data was conducted in a Geographic Information System application. GIS offers the necessary tools and flexibility to implement raster and vector-based dasymetric methods, and is used for modeling, analysis, and validation as well as for mapping and illustrating the results.

\subsubsection{Modeling spatio-temporal population distribution}

The modeling of population distribution for the LMA is based on raster dasymetric mapping using street centerlines as spatial reference units to reallocate population counts.

Table 9 Main input data sets used for modeling population distribution

\begin{tabular}{|c|c|c|}
\hline Data set & Date & Data type \\
\hline Street centerlines & 2004 & Vector polyline \\
\hline $\begin{array}{l}\text { Land use/cover maps } \\
\text { (COS90; CLC2000) }\end{array}$ & $1990 ; 2000$ & Vector polygon \\
\hline Census block groups & 2001 & Vector polygon \\
\hline Census statistics & 2001 & Database (MS Access) \\
\hline Commuting statistics & 2001 & Table (O/D matrix) \\
\hline $\begin{array}{l}\text { Daytime worker/student } \\
\text { population distribution }\end{array}$ & 2001 & Raster (25m) \\
\hline
\end{tabular}

Dasymetric mapping is a cartographic technique that allows limiting the distribution of a variable to the zones where it is present by using related ancillary information in the process of areal interpolation (Wright, 1936; Eicher and Brewer, 2001). Raster based dasymetric mapping with adequate resolution can be effective at bridging the gap between visualization-oriented choropleth maps and analysis-oriented areal interpolation. A top-down approach is 
employed to spatially disaggregate and refine the population from official census and statistics for nighttime and daytime periods. The most recent statistical and census data available (2001) provide the population counts for each daily period, while physiographic data sets define the spatial units (i.e. grid cells) used to disaggregate those counts. The model combines the approach proposed by McPherson and Brown (2004) with the innovative use of "intelligent" dasymetric mapping (Mennis and Hultgren, 2006) to disaggregate official population counts to target zones.

Four raster population distribution surfaces were produced, at $25 \mathrm{~m}$ resolution: (1) nighttime (residential) population, (2) daytime residential population, (3) daytime worker and student population, and (4) total daytime population. The basic methodology was presented and tested previously for Cascais and Oeiras, two municipalities of the LMA (Freire, 2010). However, due to being very laborintensive and exceedingly costly, the original methodology had to be adapted and improved to expedite the modeling of a much larger area with sufficient accuracy. The nighttime population distribution surface was obtained by allocating resident population from census zones to residential streets. First, relevant classes were selected from the LULC maps and combined, in order to identify residential land use. Some rules were applied to minimize the effect of errors present in the LULC data. Two residential classes were considered and sampled, using the containment method as proposed by Mennis and Hultgren (2006) to derive the respective density weights: "Continuous Urban Fabric" and "Discontinuous Urban Fabric". Then, freeways were removed from consideration and the resulting eligible streets were intersected with residential land use from LULC data to obtain residential streets. Subsequently, these were rasterized at $25 \mathrm{~m}$ resolution and the population from census block groups (source zones) was interpolated to the respective residential street cells (target zones) according to the density weights.

The total daytime population distribution results from the sum of two surfaces on a cell-by-cell basis: (1) the daytime population in their places of work or study - i.e. the workforce population surface, and (2) the population that remains home during the day - i.e. the daytime residential population grid. The latter is obtained by multiplying the nighttime distribution by the ratio of resident population who, according to official statistics (INE, 2003), does not commute to work or school in each municipality. The workforce population surface was created by allocating commuters to selected "labor" streets, in a fashion similar to the one used for modeling nighttime distribution. Two classes of varying workforce density were also defined and sampled, using the previously generated detailed workforce population surface for Cascais (Freire, 2010) to derive density weights. The resulting $25-\mathrm{m}$ population grids were aggregated to $50-\mathrm{m}$ cells for analysis and visualization purposes, thus representing densities by $2500 \mathrm{~m} 2$ (0.25 ha).

Nighttime distribution was validated using the higher-resolution census block 
units as reference (i.e. ground truth) in a correlation analysis. The corresponding correlation coefficient (Pearson's $r$ ) was 0.85 . Validation of workforce distribution was limited by lack of an independent and reliable reference data set covering the whole LMA study area. Correlating the new workforce surface in Oeiras with the database previously generated for that municipality yielded a coefficient of 0.60 . Additional details on population distribution modeling are provided in Freire (2011).

\subsubsection{Assessing population and classifying human exposure to seismic hazard}

The Seismic Intensity map was obtained from the PEERS-AML-CL (ANPC, 2007) in image format and was manually digitized and clipped for the study area (Figure 10). Using the Modified Mercalli Intensity Scale (USGS, 2009) it represents the expected intensities generated by magnitude 6.6/6.7 earthquakes with epicenter in the lower valley of the river Tagus. In order to improve the assessment of human exposure as a contribution to better characterization of seismic risk in the LMA, two analyses are implemented: (1) quantification of population potentially affected by seismic intensity levels in nighttime and daytime periods, and (2) classification and mapping of human exposure to seismic hazard in nighttime and daytime periods. Population potentially affected by seismic intensity levels is assessed using zonal analysis to summarize nighttime and daytime population surfaces by seismic zone of the earthquake intensity map. Figure 11 and Figure 12 illustrate the varying population distribution and densities in nighttime versus daytime periods in each intensity zone. 


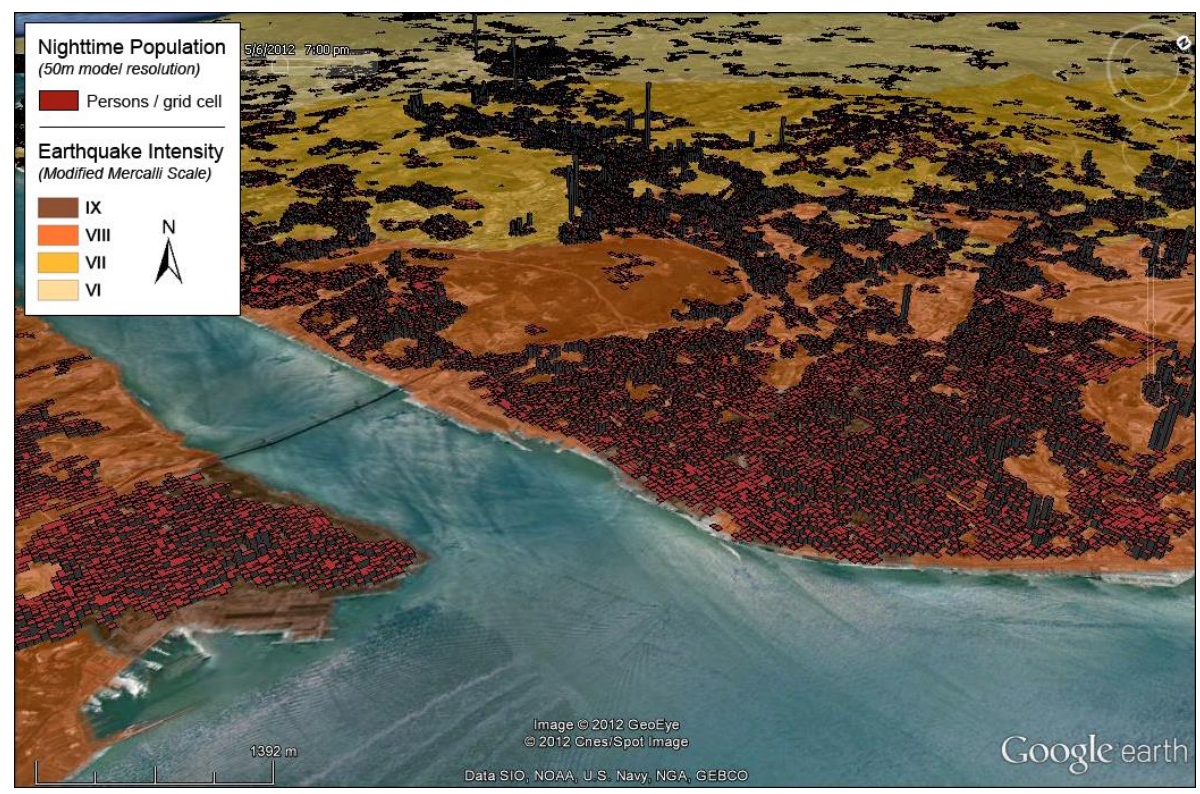

Figure 11 Nighttime population density and seismic zones (background from Google Earth).

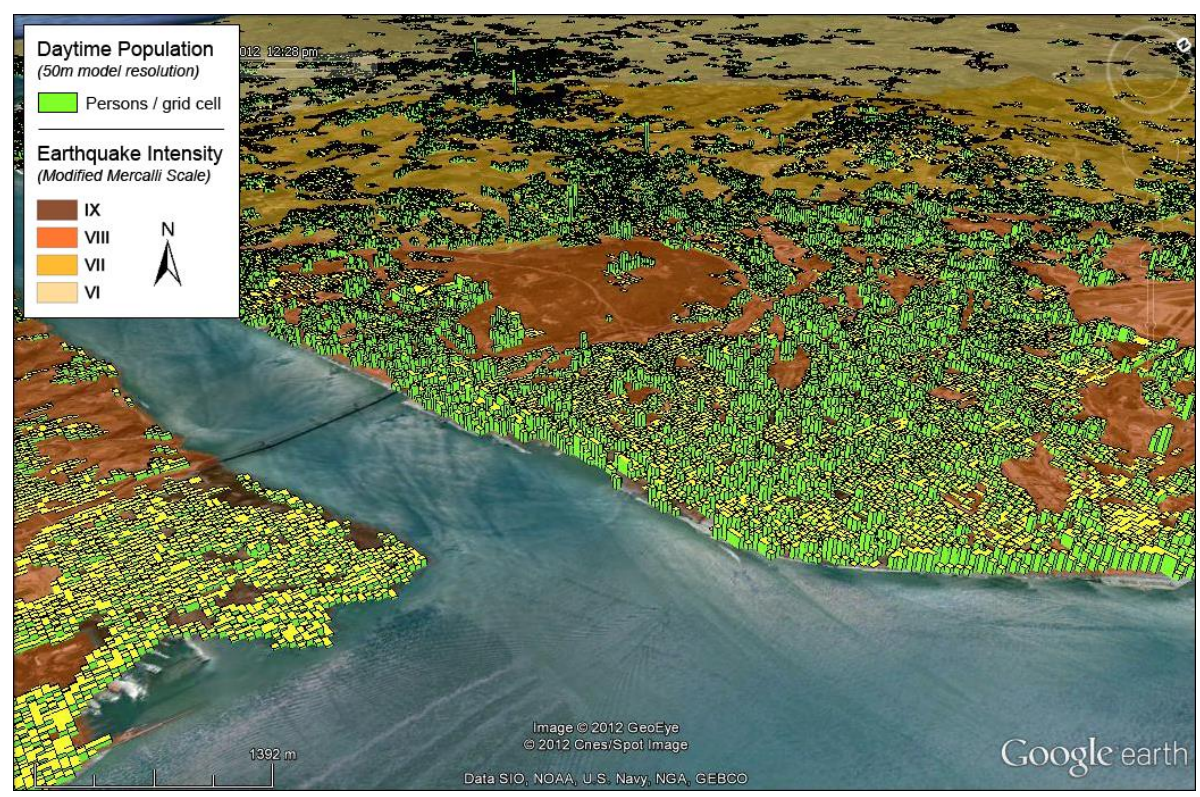

Figure 12 Daytime population density and seismic zones (background from Google Earth).

The second analysis involves defining major classes for seismic intensity and population density and corresponding subsequent reclassification. Combining 
these two variables, human exposure levels are derived, mapped, and quantified. Ranking human exposure by using just a few categories helps in having a clear perspective of its distribution. Avoiding cognitive overload is considered highly beneficial in visual risk communication (Lundgren and McMakin, 2009) and can assist in prioritizing areas for mitigation and response actions. Therefore, in order to reclassify the two variables (i.e. population density, seismic intensity) into a common and easily understandable ordinal scale, four main categories are defined: (1) Very High, (2) High, (3) Moderate, and (4) Low.

The class breaks for population density (in persons/ha) are derived based on histogram analysis and adjusted by rounding. For the seismic hazard, the whole Modified Mercalli scale, varying from I to XII, is reclassified based on intensity levels and definitions (see USGS, 2009) and by using a cautious approach, i.e. by including level IX in the highest category. Referring to the manner in which the earthquake is felt by people, the lower six levels are grouped in the Low and Moderate categories. The higher six levels, referring to observed structural damage, are classified as High and Very High. Figure 13 shows original levels and classes, corresponding categories, and combined human exposure classes. In the study area, the seismic intensity levels vary from VI to IX (cp. Figure 10).

\begin{tabular}{|c|c|c|c|c|c|c|}
\hline & & & \multicolumn{4}{|c|}{ Population Density [Persons/ha] } \\
\hline & & & 401- & $201-400$ & $101-200$ & $0-100$ \\
\hline & & Exposure Level & VH & $\mathbf{H}$ & M & $\mathbf{L}$ \\
\hline \multirow{12}{*}{ 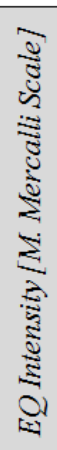 } & $\mathrm{XII}$ & VH & $\mathrm{VH}$ & $\mathrm{VH}$ & $\mathrm{H}$ & $\mathrm{M}$ \\
\hline & $\mathrm{XI}$ & VH & $\mathrm{VH}$ & $\mathrm{VH}$ & $\mathrm{H}$ & $\mathrm{M}$ \\
\hline & $\mathrm{X}$ & $\mathrm{VH}$ & $\mathrm{VH}$ & $\mathrm{VH}$ & $\mathrm{H}$ & $\mathrm{M}$ \\
\hline & IX & $\overline{\mathrm{VH}}$ & $\mathrm{VH}$ & $\overline{\mathrm{VH}}$ & $\overline{\mathrm{H}}$ & $\bar{M}$ \\
\hline & VIII & $\mathrm{H}$ & $\mathrm{VH}$ & $\mathrm{H}$ & $\mathrm{H}$ & $\mathrm{M}$ \\
\hline & VII & $\mathrm{H}$ & $\mathrm{VH}$ & $\mathrm{H}$ & $\mathrm{H}$ & $\mathrm{M}$ \\
\hline & VI & M & $\mathrm{H}$ & $\mathrm{H}$ & $\mathrm{M}$ & $\mathrm{M}$ \\
\hline & $\mathrm{V}$ & $\bar{M}$ & $\overline{\mathrm{H}}$ & $\overline{\mathrm{H}}$ & $\bar{M}$ & $\bar{M}$ \\
\hline & IV & M & $\mathrm{H}$ & $\mathrm{H}$ & $\mathrm{M}$ & $\mathrm{M}$ \\
\hline & III & $\mathbf{L}$ & $\mathrm{M}$ & $\mathrm{M}$ & $\mathrm{M}$ & $\mathrm{L}$ \\
\hline & II & $\mathbf{L}$ & $\mathrm{M}$ & $\mathrm{M}$ & $\mathrm{M}$ & $\mathrm{L}$ \\
\hline & I & L & M & $\mathrm{M}$ & $\mathrm{M}$ & $\mathrm{L}$ \\
\hline
\end{tabular}

VH (very high), H (high), M (moderate), L (low)

Framed in black: Earthquake intensity classes in the study area

\section{Figure 13 Classification approach to categorize human exposure levels}

The reclassified seismic intensity map is rasterized at $50 \mathrm{~m}$ resolution and combined with the reclassified nighttime and daytime population density maps, resulting in maps of human exposure to seismic hazard for each of those periods. We use the two-color grading for symbolization, as recommended by Gaspar-Escribano and Iturrioz (2011) for this type of risk communication 
(Figure 14 and Figure 15). Total population and area are then summarized for the resulting human exposure categories in the LMA. 


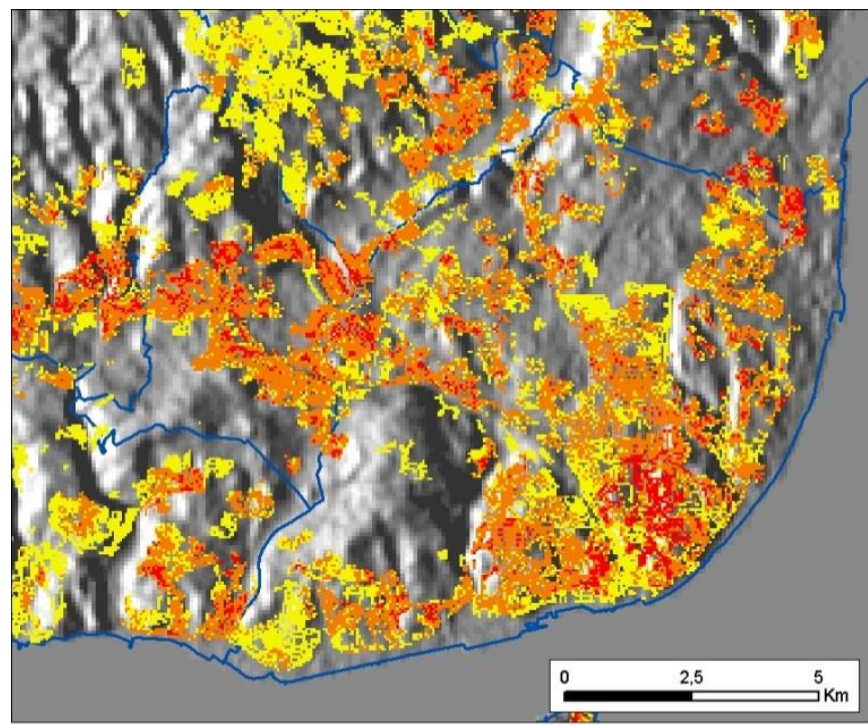

\begin{tabular}{|c|c|}
\hline Nighttime Human Exposure & \\
\hline high & $\begin{array}{l}\text { and modeled population distribution. } \\
\text { Unpopulated places are left blank. }\end{array}$ \\
\hline
\end{tabular}

Figure 14 Map of nighttime human exposure to seismic hazard

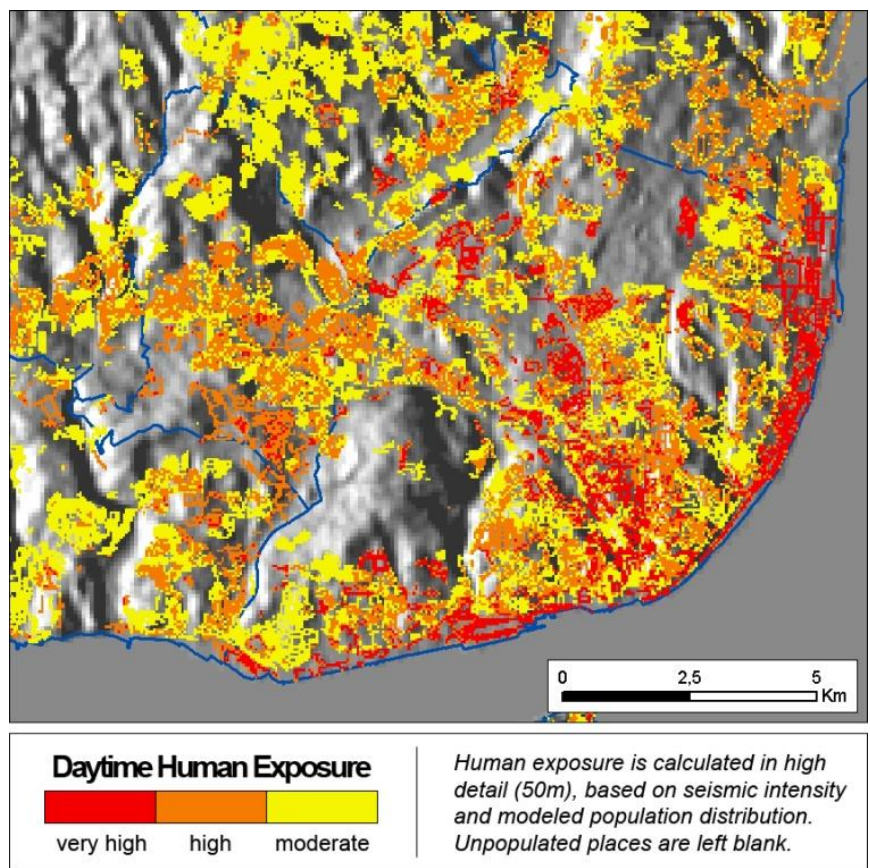

Figure 15 Map of daytime human exposure to seismic hazard 


\subsection{Results and discussion}

The modeled population surfaces represent maximum expected densities on a typical workday, assuming that everyone is at home at night and all workers and students are in their workplaces and schools, and the remainder in their residences during the daytime period. While this is a simplification of reality, it is a major improvement over residence-based data sets that can benefit analyses from regional to local scale. Although in each surface only total population is modeled, it is an essential baseline indicator for first assessment of exposure to any type of (natural and/or man-made) hazard. After the disaggregation process, the model preserves the official population counts used as input.

Table 10 quantifies the total population potentially exposed to each seismic intensity level in the nighttime and daytime periods. It shows that from night to day the population exposed to the two highest seismic levels increases, while the number of persons exposed to the two lower levels decreases. More specifically, exposure to the highest seismic level (i.e. level IX) increases by $22 \%$ to affect $5 \%$ of the total daytime population (137,222 people). Even more important, from the nighttime to daytime period an additional 204786 persons are exposed to the levels VIII and IX, which then contain $52 \%$ of the daytime population. This is due to the shift in population distribution and the concentration of daytime activities in areas of higher seismic intensity. The level VIII zone concentrates the largest share of the population both in nighttime and daytime periods, while not occupying the largest share of the surface of the LMA.

The area and total population in each human exposure level in nighttime and daytime periods are presented in Table 11. It shows that most of the area and population of the LMA are in Moderate or High exposure classes in both time periods. However, while only $3 \%$ of the populated area is classified as Very High exposure, this class includes $23 \%$ of the total population in the daytime period. This represents an increase of $48 \%(203,641)$ in population and also $31 \%$ in area from nighttime to daytime. It also indicates a significant increase in population density in that exposure class between those periods. Despite the high temporal consistency of input data sets, the main source of inaccuracies for the total daytime population distribution surface lies in the daytime worker and student population grid, with the model propagating error and uncertainties present in the input data. The locations of active commercial and industrial sites can easily become outdated, as these activities are usually more dynamic than residential areas, and "hybrid" LULC maps based on remote sensing data are notoriously problematic at capturing effective land use. Also, there is no indication of workers' density in these land use patches, and disaggregating the population based on the surface of their street network leads to inaccuracies. One major limitation is the availability of mobility statistics (and derived number of workers and students) only at the municipal 
level, resulting in uncertainty as to their actual distribution within the municipality. Finally, density weights used for interpolating the workforce to "labor" land use classes are obtained from one municipality (Cascais) that may not be similar to others in the LMA.

Table 10 Population exposed to seismic intensity levels in nighttime and daytime periods in the study area

\begin{tabular}{|c|c|c|c|}
\hline \multirow{2}{*}{$\begin{array}{l}\text { Earthquake intensity } \\
\text { [M. Mercalli S.] }\end{array}$} & \multicolumn{2}{|c|}{ Population } & \\
\hline & Absolute [Pers.] & Relative [\%] & \\
\hline IX & 112,826 & 4 & \\
\hline VIII & $1,076,180$ & 41 & $z$ \\
\hline VII & 887,493 & 34 & 产 \\
\hline VI & 569,940 & 22 & \\
\hline Total & $2,646,439$ & 100 & \\
\hline IX & 137,222 & 5 & \\
\hline VIII & $1,256,570$ & 47 & \\
\hline VII & 746,992 & 28 & ָָ \\
\hline VI & 535,767 & 20 & \\
\hline Total & $2,676,551$ & 100 & \\
\hline IX & 24,396 & 22 & \\
\hline VIII & 180,390 & 17 & 吾 \\
\hline VII & $-140,501$ & -16 & $\frac{10}{10}$ \\
\hline VI & $-34,173$ & -6 & 岂 \\
\hline Total & 30,112 & 1 & (1) \\
\hline
\end{tabular}

Relative differences are relative to the night numbers. Sums can add up to more than $100 \%$ due to rounding.

Table 11 Total surface and population in each human exposure class in nighttime and daytime periods in the study area

\begin{tabular}{|c|c|c|c|c|c|}
\hline \multirow[t]{2}{*}{ Human Exposure } & \multicolumn{2}{|c|}{ Area } & \multicolumn{2}{|c|}{ Population } & \\
\hline & Abs. [ha] & Rel. [\%] & Abs. [Pers.] & Rel. [\%] & \\
\hline $\mathrm{VH}$ & 884 & 3 & 423,112 & 16 & \\
\hline $\mathrm{H}$ & 6,390 & 21 & $1,308,780$ & 49 & $\underline{a}$ \\
\hline$M$ & 22,617 & 76 & 914,550 & 35 & 岂 \\
\hline Total & 29,891 & 100 & $2,646,442$ & 100 & \\
\hline $\mathrm{VH}$ & 1,154 & 3 & 626,753 & 23 & \\
\hline $\mathrm{H}$ & 6,022 & 17 & $1,062,020$ & 40 & סु \\
\hline$M$ & 27,611 & 79 & 987,772 & 37 & \\
\hline Total & 34,787 & 100 & $2,676,545$ & 100 & \\
\hline $\mathrm{VH}$ & 270 & 31 & 203,641 & 48 & \\
\hline $\mathrm{H}$ & -368 & -6 & $-246,760$ & -19 & $\underset{D}{=}$ \\
\hline$M$ & 4,994 & 22 & 73,222 & 8 & $\stackrel{\rightrightarrows}{\rightleftarrows}$ \\
\hline Total & 4,896 & 16 & 30,103 & 1 & \\
\hline
\end{tabular}

VH (very high), H (high), M (moderate), L (low)

Relative differences are relative to the night numbers 


\subsection{Conclusions}

An approach was developed that enables modeling and mapping of spatiotemporal population distribution and density in the daily cycle at high spatial resolution to advance analysis of earthquake exposure and eventually improve risk assessment. Benefits of this approach were illustrated with application to a large metropolitan area prone to this type of hazard. By combining land use data sets and demographic census and mobility statistics, the population model yields mapping of earthquake risk. Analysis of exposure to seismic levels in the LMA shows that there are considerable differences from nighttime to daytime, with significantly more people potentially at risk in the daytime period. This fact implies that conducting exposure analysis based on census data alone may result in misestimating risk for a daytime event, such as the great 1755 earthquake, with possible serious consequences for response and evacuation activities.

Since population density is a crucial factor determining earthquake losses, the refined spatio-temporal population surfaces were combined with a recent seismic intensity map to derive new spatial representations of human exposure. The new maps are spatially-explicit four-class-composites of human exposure to seismic intensity mainly aimed at benefiting visualization and communication of earthquake risk, which can eventually contribute to better decision-making in a disaster management context. Results show that very little of the populated area is classified as Very High exposure and yet it includes almost a quarter of the daytime population. Although the bestavailable seismic map for the study area has lower spatial detail than the population surfaces, their raster structure allows enhanced flexibility, rapid assessment of exposure and easy integration with improved hazard maps, when compared to the typical vector-based census data. Additionally, such population distribution data sets can be combined with different hazard maps to improve spatio-temporal exposure assessment and mapping for any type of hazard, natural or man-made, and at any scale. This effort is a contribution to address recent recommendations to enhance vulnerability analyses (Cutter, 2003; Balk et al., 2006; Birkmann, 2007; NRC, 2007). Such an improved characterization of vulnerability and risk can benefit all phases of the disaster management process where human exposure should be considered, namely in emergency planning, risk mitigation, preparedness, and response to an event. Despite their inherent uncertainties, addressed above, these results can be used at the planning stage to improve characterization of potential population exposure in the hazard zones, and detailed population surfaces can also be used as input in earthquake simulators for modeling of human casualties in different hazard scenarios. Risk mitigation measures might include lowering human exposure levels by decreasing population densities in future urban planning decisions, for which a critical assessment of current exposure is needed. During the preparedness stage, means and resources needed for 
response can be placed according to exposure levels, or these areas can be prioritized for evacuation training. After the event, a quick estimation of affected people and potential victims is vital for tailoring response and rescue efforts, including allocating emergency personnel, hospital beds, and other resources (Freire, 2010). Population models can be further improved by using more up-to-date and detailed land use/land cover data detailing functional use, ideally by city block or building (Aubrecht et al., 2009). This improvement was already demonstrated for part of the study area in the context of exposure to tsunami (Freire et al., 2012). The availability of finer-level commuting statistics (e.g. at the level of commune) would greatly reduce uncertainties in the daytime scenario. Concerning temporal resolution, it would be important to represent weekly and seasonal cycles, which affect population distribution in many urban areas. This development is limited by the unavailability of mobility statistics that enable characterization of these cycles. Future work should also focus on evolving from mapping of human exposure to social (and economic) vulnerability, thus incorporating certain indicators picturing social and economic characteristics, a great challenge at such detailed resolution. Perhaps a less daring development would be to combine structural vulnerability of buildings, when available, with estimates of population present to better approximate potential human losses in case of an earthquake.

\section{Acknowledgements:}

We thank Nuno Gomes for assisting with preparation of datasets. The publication of this paper was supported in part by the Strategic Project of eGEO (PEstOE/SADG/UI0161/2011) Research Centre for Geography and Regional Planning, funded by the Portuguese State Budget through the Fundacao para a Ciencia e a Tecnologia. 


\section{Chapter 4. Advancing tsunami risk assessment by improving spatio-temporal population exposure and evacuation modeling ${ }^{36}$}

36 This chapter is published as:

Freire, S., C. Aubrecht, S. Wegscheider, 2013. Advancing tsunami risk assessment by improving spatio-temporal population exposure and evacuation modeling. Natural Hazards, 68:1311-1324. 
Advancing tsunami risk assessment by improving spatio-temporal population exposure and evacuation modeling

\begin{abstract}
Tsunamis are among the most destructive and lethal of coastal hazards. These are time-specific events, and despite directly affecting a narrow strip of coastline, a single occurrence can have devastating effects and cause massive loss of life, especially in urbanized coastal areas. In this work, in order to consider the time dependence of population exposure to tsunami threat, the variation of spatio-temporal population distribution in the daily cycle is mapped and analyzed in the Lisbon Metropolitan Area. High resolution daytime and nighttime population distribution maps are developed using 'intelligent dasymetric mapping,' that is, applying areal interpolation to combine bestavailable census data and statistics with land use and land cover data. Workplace information and mobility statistics are considered for mapping daytime distribution. In combination with a tsunami hazard map, information on infrastructure, land use and terrain slope, the modeled population distribution is used to assess people's evacuation speed, applying a geospatial evacuation modeling approach to the city of Lisbon. The detailed dynamic population exposure assessment allows producing both daytime and nighttime evacuation time maps, which provide valuable input for evacuation planning and management. Results show that a significant amount of population is at risk, and its numbers increase dramatically from nighttime to daytime, especially in the zones of high tsunami flooding susceptibility. Also, full evacuation can be problematic in the daytime period, even if initiated immediately after a major tsunami-triggering earthquake. The presented approach greatly improves tsunami risk assessment and can benefit all phases of the disaster management process.
\end{abstract}

\title{
4.1 Introduction
}

Tsunamis are sudden-onset natural hazards having a short duration, yet capable of producing lasting impacts of local to regional geographical scope (Peduzzi 2004). Despite being relatively rare events, large tsunamis are among the most destructive and lethal of coastal hazards, with a single occurrence having the potential to cause huge loss of life (Nirupama 2009), as recently again demonstrated, for example, in December 2004 in the Indian Ocean and in 2011 in Tohoku, Japan.

Comprehensive assessment and mapping of communities' risk to natural hazards, such as tsunamis, requires estimation of social vulnerability, of which population exposure is considered one of the most critical variables and preassessment requisites (Aubrecht et al. 2012b). However, more effort has been put into understanding of tsunami hazard than into estimating potential impacts on people and infrastructure (Wood 2007; Jelinek et al. 2012), despite quantitative assessment of tsunami risk being necessary to support spatial planning and for local authorities to provide population protection (Lima et al. 2010). Therefore, step one of tsunami preparedness includes assessing and 
mapping concentrations of population present (NSTC 2005; IOC 2008), since for practical purposes, one can consider all human beings to be equally vulnerable in case of direct tsunami impact (Villagran de Leon 2008). Population data are a basic necessity for assessing potential human exposure, with its quality and level of detail having a direct effect on response and lives saved (NRC 2007). Furthermore, updated and detailed mapping of population distribution is important for decision support in practically every phase of the emergency management cycle, if produced at appropriate spatial and temporal scales (Sutton et al. 2003).

On November 1,1755 , following a large earthquake $(M=8.5)$, the city of Lisbon was hit by a major tsunami having 6-m high waves that caused much loss of life (Baptista et al. 1998; Chester 2001). This very destructive event occurred during the daytime period, sometime after 10 a.m., lasting for only a few minutes. Tsunamis are quick-onset hazards usually having a short duration, whose lead time is strongly dependent on the existence of an earlywarning system.

The spatial distribution of population, and hence exposure to hazards, is also time dependent, especially in large urban areas. Due to human activities and mobility, the distribution and density of population vary greatly in the daily cycle. Therefore, a more accurate assessment of population exposure and risk analysis requires going beyond residence-based census maps and figures (Aubrecht et al. 2012a). The development of the LandScan Global Population Database (Dobson et al. 2000) represented a great improvement over residence-based population data sets. However, its spatial resolution (30 arcseconds) is still too coarse to adequately support analyses at the local level, and the representation of 'ambient population' corresponds to a temporal averaging that is not ideal for using in time-specific hazards, such as a tsunami, which can strike a limited area at any time of day or night. To address these limitations, generic population distribution databases having higher temporal and spatial detail are being developed for the territory of the USA (Bhaduri et al. 2002; McPherson and Brown 2004). In the context of the development of a tsunami early-warning system for Indonesia, Taubenbock et al. (2009) have improved risk analysis for Padang, West Sumatra, by assessing hazard and vulnerability, including population and evacuation modeling, however adopting a bottom-up, survey-based extrapolation. Results showed a difference in population exposure from nighttime to daytime, even if only a slight increase $(4.5 \%)$. Since tsunamis are time-specific events which usually directly affect a narrow strip of coastline, advanced assessment of human exposure and integrated risk analysis requires spatially detailed demographic data which account for population dynamics typical of urban areas (Freire et al. 2011). The Lisbon Metropolitan Area (LMA), Portugal, is subject to considerable likelihood of tsunami, as confirmed by the occurrence of numerous events in the past, including several minor ones in the twentieth century (Baptista and 
Advancing tsunami risk assessment by improving spatio-temporal population exposure and evacuation modeling

Miranda 2009). Although the probability of occurrence is lower than other natural hazards, impacts can be extremely high and tsunamis are a major risk for Lisbon coastal areas (Baptista et al. 2006). Tsunami hazard is usually represented by inundation maps that identify areas and depths of tsunami flooding or run-up. The Regional Plan for Territorial Management for the Lisbon Metropolitan Area (PROTAML), recently under discussion, includes a Tsunami Inundation Susceptibility map for the area, showing that significant urbanized areas may be at risk (CCDR-LVT 2010). However, no assessment of vulnerability or human exposure to this hazard was conducted in the framework of the plan.

The present work aims at improving the assessment of tsunami risk and contributing to more efficient and effective emergency management (EM) by considering the time dependence of population exposure to this hazard in a large urban area. Population density is modeled in high spatial and temporal detail following a top-down approach to disaggregate data from official statistics. The resulting population distribution surfaces are integrated with the above-mentioned tsunami hazard map (from PROTAML) to estimate potential human exposure. Additionally, the population's speed of evacuation is modeled and analyzed in the nighttime and daytime periods to investigate whether there are significant differences and what consequences these might have for EM.

\subsection{Data and study area}

\subsubsection{Study area}

The study area for modeling spatio-temporal population distribution and assessing its exposure to tsunami is the Lisbon Metropolitan Area (LMA) (Figure 16). 


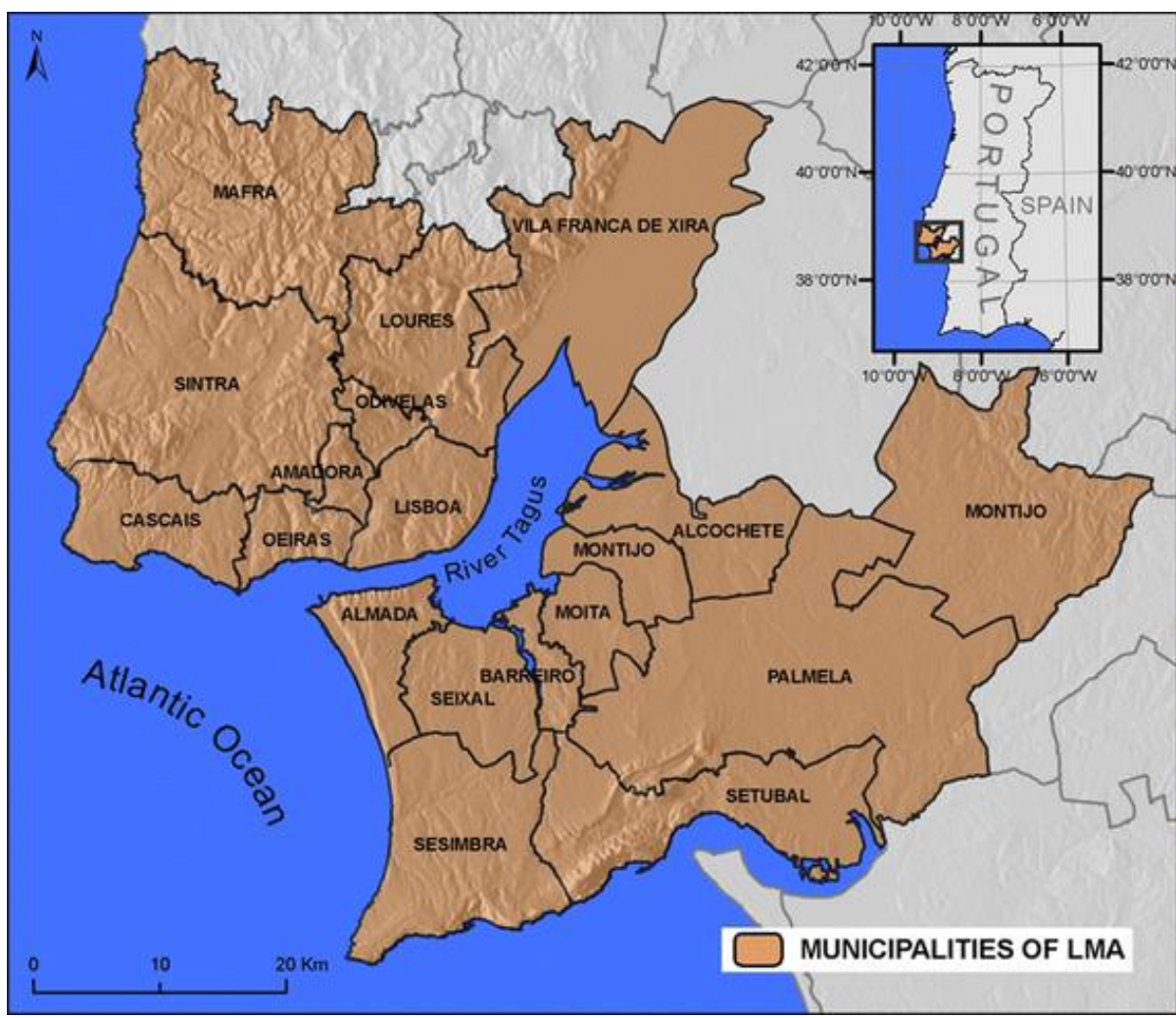

Figure 16 Study area - Lisbon Metropolitan Area (LMA)

The LMA is the main metropolitan area in Portugal and includes eighteen municipalities. About $30 \%$ of all national companies are headquartered in this region, which is responsible for $36 \%$ of the country's GDP. The LMA has been experiencing growing urbanization along its coastline, with artificial surfaces increasing by $36 \%$ in the $20-\mathrm{km}$ coastal zone between circa 1990 and 2000 (Freire et al. 2009). The LMA occupies a total land area of 2,963 km2, and its resident population has increased by $5.6 \%$ from 1991 to 2001 , to a total of $2,661,850$ residents, $26 \%$ of the country's population (INE 2001). This results in an average population density of 898 inhabitants per square kilometer, but these densities vary significantly in space and time. Beyond the more urbanized core and densely populated zones, the LMA still includes vast rural areas with dispersed settlements whose uneven population density is not adequately captured and represented by census polygons. Some of these population enumeration units can be quite large even at the block level, which is the smallest census reporting unit. Moreover, the daytime population of municipalities in the metro area of Lisbon can surpass by more than $50 \%$ the residential figures from the census, due to significant daily commuting for work and study (INE 2003). The geographical situation and characteristics of the 
Advancing tsunami risk assessment by improving spatio-temporal population exposure and evacuation modeling

LMA, bordering the Atlantic Ocean and the large estuary of the Tagus River, and being prone to tsunamis, provide an appropriate context for this research.

\subsubsection{Data sets}

The main data sets produced and used in the course of the presented analyses were population distribution surfaces and a Tsunami Inundation Susceptibility map (Figure 17). Input variables used for modeling population distribution include both physiographic and statistical data.

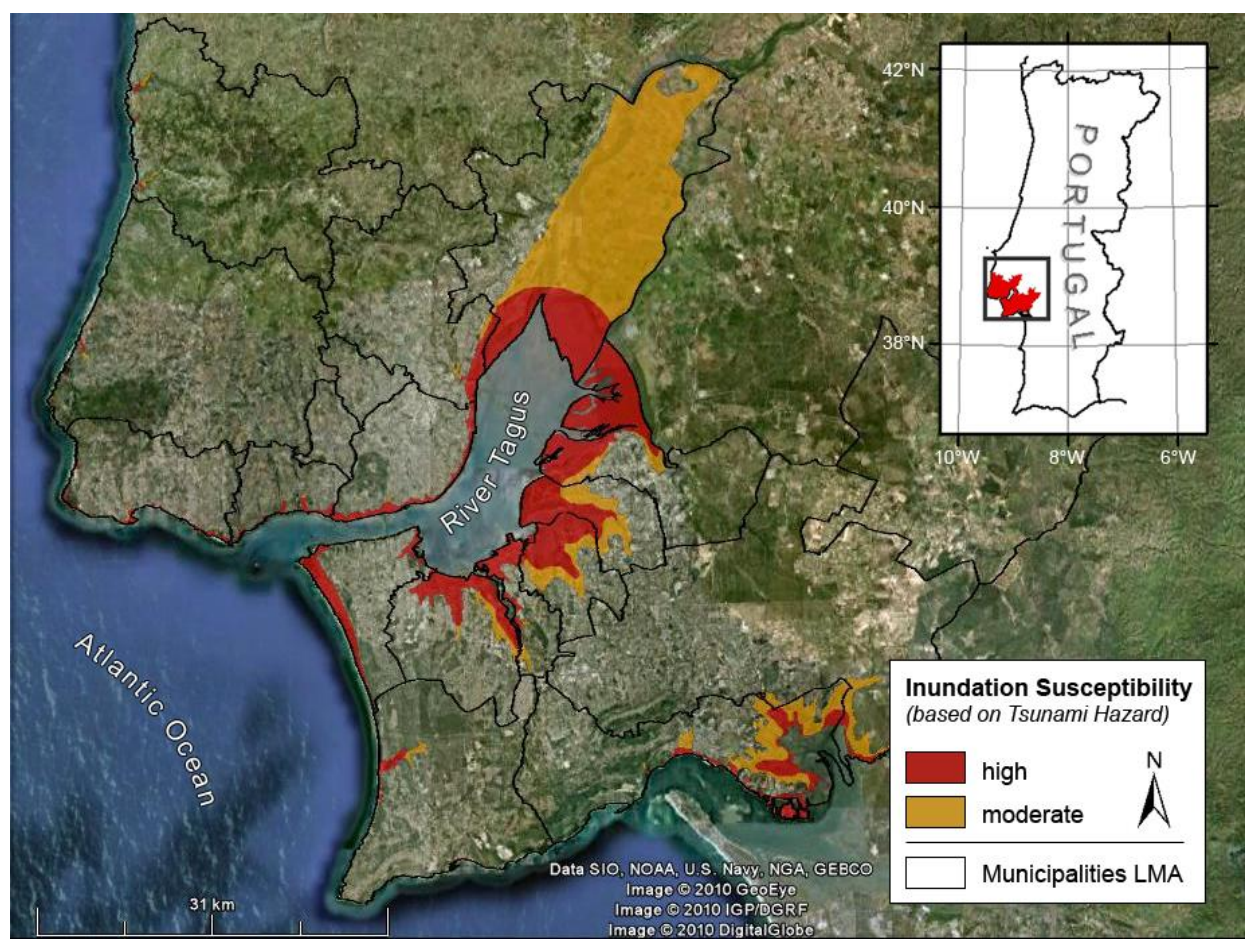

Figure 17 Tsunami Inundation Susceptibility map for the LMA (background from Google Earth)

The first group comprises street centerlines and land use and land cover (LULC) maps; the second set includes official census counts (INE 2001), data on workforce, and commuting statistics (INE 2003) for the study area, produced by Statistics Portugal. Commuting statistics were obtained in the form of an origin/destination matrix of daily commuters at municipal level for the LMA, from which the number of workers and students present in each municipality was derived. The used data were obtained from various sources and in different formats which are listed in Table 12. It was assured that all data sets were in the same projected coordinate system. COS90 (Carta de Ocupação do Solo, i.e., land cover map) is a digital LULC map at the scale 1:25,000 covering almost the entire country; however, it dates from 1990. Therefore, to ensure 
near-perfect temporal consistency among input data sets, it was decided to update it to some extent using the more recent CORINE Land Cover database for the year 2000 (CLC2000). This updating consisted in adding the new patches of artificial surfaces appearing between 1990 and 2000.

Table 12 Main input data sets used for modeling population distribution

\begin{tabular}{|c|c|c|c|}
\hline Data set & Source & Date & Data type \\
\hline \multirow[t]{2}{*}{ Street centerlines } & Private & 2004 & Vector polyline \\
\hline & vendor & & \\
\hline Land use/cover maps & Public & 1990; & Vector polygon \\
\hline$(\operatorname{COS} 90 ;$ CLC2000) & & 2000 & \\
\hline Census block groups & Public & 2001 & Vector polygon \\
\hline \multirow[t]{2}{*}{ Census statistics } & Public & 2001 & Database \\
\hline & & & (MS Access) \\
\hline \multirow[t]{2}{*}{ Commuting statistics } & Public & 2001 & Table \\
\hline & & & (O/D matrix) \\
\hline Daytime worker/student & Previous & 2001 & Raster (25 m) \\
\hline population distribution for & study (Freire & & \\
\hline Cascais and Oeiras & 2010) & & \\
\hline
\end{tabular}

The map of tsunami hazard for the LMA was produced for the Regional Plan for Territorial Management for the Lisbon Metropolitan Area (PROTAML) report (CCDR-LVT 2010) and was obtained in digital vector format. This map results from modeling an event similar to the 1755 occurrence as a worst-case scenario and depicts areas susceptible to inundation by tsunami (i.e., inundation extent). Susceptibility is represented using two classes or levels, High and Moderate, with the former including low-lying coastal areas and the latter referring to adjacent low-elevation areas farthest from the coast, with decreasing likelihood of inundation by tsunami (Figure 17). This map can be considered to represent maximum generic tsunami hazard for the LMA, given that small variations in the characteristics of the tsunami source may not be too significant for impact assessment (Lima et al. 2010).

\subsection{Methodology}

\subsubsection{Modeling nighttime and daytime population distribution}

Detailed population distribution for the LMA is modeled based on raster dasymetric mapping using street centerlines as spatial reference units to reallocate population counts. Dasymetric mapping is a cartographic technique 
Advancing tsunami risk assessment by improving spatio-temporal population exposure and evacuation modeling

which enables limiting the presence of a variable, such as population, to zones where it is present, as defined by related ancillary data (Wright 1936). In this model, the most recent statistical and census data available (2001) provide the population counts to be disaggregated, while physiographic data sets are selected and combined to define the spatial units (i.e., grid cells) used to disaggregate those counts. The approach applied by McPherson and Brown (2004) is combined with the innovative method of 'intelligent dasymetric mapping' proposed by Mennis and Hultgren (2006) in order to transfer population counts from original source zones to target zones. To obtain the nighttime (residential) population distribution surface, the existing detailed census data (INE 2001) is further refined by reallocating residential population to effective residential areas. This procedure was initiated by identifying and selecting strict residential land use from the updated LULC map. Two residential classes ('Continuous Urban Fabric' and 'Discontinuous Urban Fabric') were considered and sampled to derive the respective population density weights, using the containment method proposed by Mennis and Hultgren 2006. Then, eligible streets (i.e., all except freeways) were intersected with residential land use from LULC data to obtain residential streets, which were rasterized at $25-\mathrm{m}$ resolution. Finally, the population from census block groups (source zones) was interpolated to the respective residential street cells (target zones) according to the density weights.

The total daytime population distribution grid results from the sum of two surfaces on a cell-by-cell basis: (1) the daytime population in their places of work or study-the workforce population surface-and (2) the population that remains home during the day - the daytime residential population grid. This latter grid is obtained by applying to the nighttime (i.e., residential), the ratio of people who do not commute for study or work, in each municipality, according to the population mobility statistics (INE 2003). The workforce population surface is produced by interpolating the total number of workers and students in each municipality (source zone) to rasterized streets within 'labor' and mixed 'labor-residential' land-use polygons (target zones). While 'labor' polygons were simply extracted by selecting appropriate LULC classes, the mixed 'labor-residential' class was defined by applying simple rule-based decisions to the census data and the LULC map. The empirical parameters used for interpolation of this surface are obtained from a previous modeling effort for part of the study area, which is based on precise geocoding of workplaces' addresses and workforce (Freire 2010). Finally, the obtained $25-\mathrm{m}$ population density grids were aggregated to $50-\mathrm{m}$ cells for subsequent analysis and visualization purposes. Although this approach was developed and presented in more detail in Freire and Aubrecht (2010, 2012 - Chapter 3), both the input data and modeling rules were refined, increasing final accuracy: Misclassification of a few areas in the LULC map were corrected, and rules used to define the mixed 'labor-residential' class were improved. 


\subsubsection{Assessing population exposure to tsunami}

In order to improve the assessment of human exposure and tsunami risk in the LMA, two analyses were implemented: (1) quantification of population exposed to tsunami inundation levels in nighttime and daytime periods, and (2) modeling evacuation in nighttime and daytime periods for a subset of the study area. Population exposure to tsunami in the LMA was assessed in a GIS environment using zonal analysis to summarize nighttime and daytime population surfaces by each susceptibility zone of the Tsunami Inundation Susceptibility map. Figure 18 illustrates in 3-D for part of the study area the varying population distribution and densities in nighttime $(A)$ versus daytime (B) periods in each tsunami susceptibility zone.
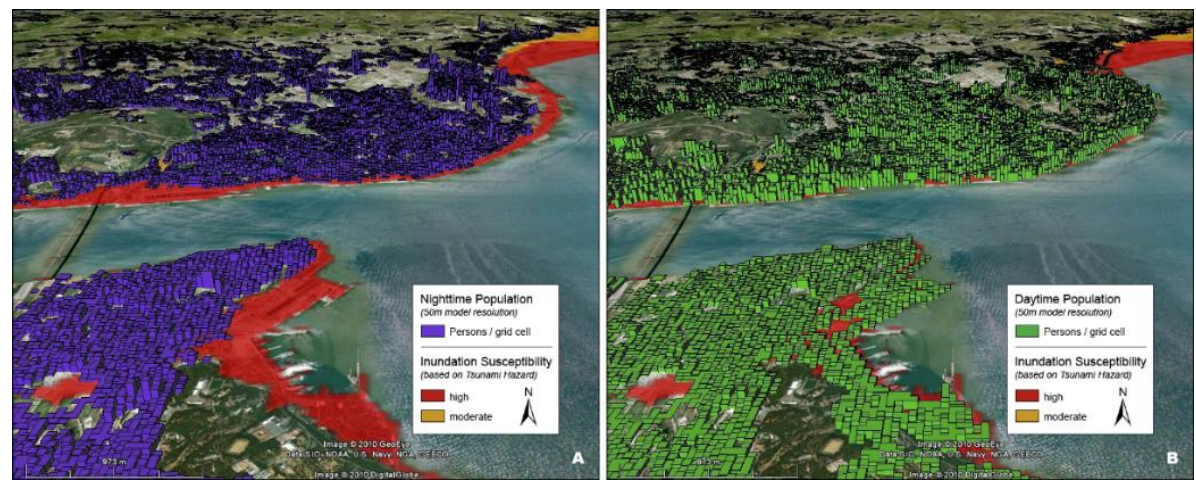

Figure 18 Nighttime $(A)$ and daytime (B) population density and Tsunami Inundation Susceptibility zones (background from Google Earth)

The modeled population surfaces represent expected maximum densities on a regular workday. In the absence of additional information, it was assumed that at night all residents are at home, and during the daytime period all workers and students are in their workplaces and schools, while the remaining population stays in their residences. Although this is still a simplification of a more complex reality concerning population distribution, it is a major improvement over existing approaches and data sets (i.e., residential census) that can benefit analyses from regional to local scale.

\subsubsection{Tsunami evacuation modeling}

An additional analysis, aimed at informing the EM planning, departs from the spatiotemporal population distribution patterns and potential tsunami inundation zones to model and estimate evacuation speed in nighttime and daytime periods. This demonstration is conducted for three freguesias (communes) of the municipality of Lisbon, susceptible to inundation by tsunami, which are located in the western part of the city: Santa Maria de Belem, Ajuda and Alcantara (Figure 19). These communes are rather heterogeneous regarding land use and joint presence of residential areas and 
Advancing tsunami risk assessment by improving spatio-temporal population exposure and evacuation modeling

economic activities, therefore providing a good test bed for this demonstration. The evacuation analysis assesses the time needed, after a full evacuation is initiated, for the population to reach safe areas, that is, areas immediately outside of the inundated zone, assuming they are traveling by foot. Part of the methodology was developed in the framework of the German-Indonesian Tsunami Early Warning System (GITEWS) project (Post et al. 2009; Strunz et al. 2011; Wegscheider et al. 2011). The evacuation modeling is performed using GIS-based geospatial analysis and relies on a cost-weighted distance approach. The best (i.e., the fastest) evacuation route from any given location to a safe area is defined, and the time needed to reach it is calculated.

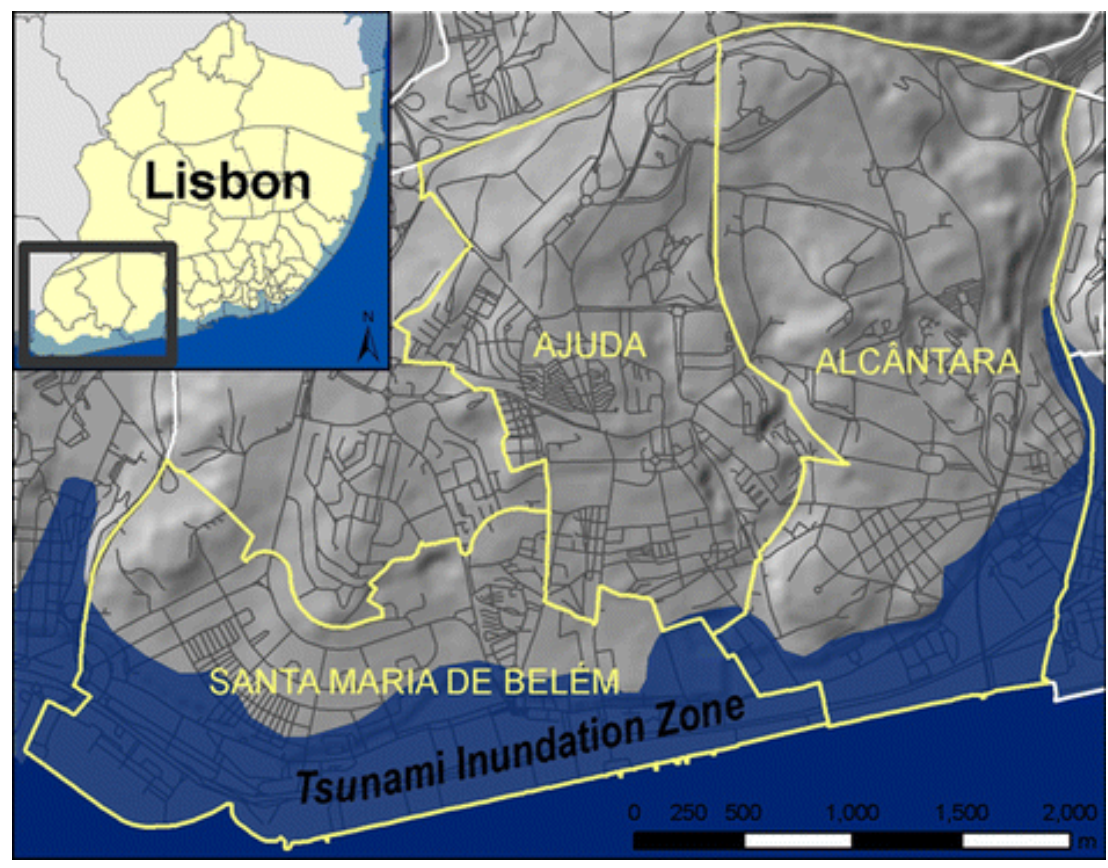

Figure 19 Study area for Tsunami evacuation modeling

The output of the model, that is, the time needed for evacuation toward a safe area, is based on several parameters: (1) extent of the hazard impact area (i.e., potential inundation area), (2) characteristics of the evacuation paths (slope, land cover, street network), (3) (time-specific) population density and (4) location of critical facilities (i.e., facilities with people of reduced or lacking abilities to evacuate, such as hospitals). For more details see Post et al. (2009) and Wegscheider et al. (2011). Data from the Urban Atlas (www.eea.europa.eu/data-and-maps/data/urban-atlas) that recently became available were used as source of land cover information. The Urban Atlas project maps land cover and land use for selected European cities (i.e., including the part of the LMA chosen for the tsunami evacuation modeling case study) using 19 thematic classes and has a minimum mapping unit of 0.25 ha 
(for 'Artificial surfaces'). While not being available for the entire LMA, it is a more detailed and updated source of LULC info for intra-urban analysis than COS90 + CLC2000. The derived evacuation time surfaces are used, in combination with the detailed population distribution grids, to calculate the number of successful evacuees after certain time intervals.

\subsection{Results and discussion}

Using the above-described intelligent dasymetric mapping methodology, four raster population distribution surfaces were produced, at $50 \mathrm{~m}$ resolution: (1) nighttime (residential) population, (2) daytime residential population, (3) daytime worker and student population, and (4) total daytime population. The nighttime population distribution is finer than the one provided by the original census data, and the other three population grids were previously unavailable. The new nighttime and workforce distribution grids were validated using correlation analysis with higher-resolution reference data, that is, block-level census and the previous' model distribution for Oeiras, respectively. Correlation coefficients (Pearson's r) of 0.86 and 0.64 , respectively, were obtained for each of these two population grids.

Concerning the population distribution model, an additional source of uncertainties lies in the spatial nature and dynamic character of activities used to produce the daytime worker and student population grid, combined with the unavailability of mobility statistics more detailed than at the municipal level. Still, the usefulness of earlier versions of similar spatio-temporal population distribution surfaces for the LMA, which are multi-purpose, was illustrated for Disaster Risk Management applications, specifically in improving earthquake risk assessment (Freire and Aubrecht 2010, 2012). Population surfaces at such high resolution prove more appropriate for local-level quantification of human exposure, enabling a more detailed and thorough assessment of potential effects and impacts. Results of the analysis of exposure to tsunami in the LMA show that the population potentially exposed to some level of tsunami inundation hazard significantly increases from nighttime to daytime periods (Table 13). The majority of the population exposed is associated with the 'high inundation susceptibility' zone. Particularly these 'high hazard' areas also feature a significant increase in population from nighttime to daytime, both in absolute and in relative terms (i.e., as proportion of total population within the inundation zone). While during nighttime around $60 \%$ of the potentially exposed population is located in areas having a high tsunami hazard level, daytime population movement results in more than 200,000 persons additionally exposed in that area, corresponding to a factor of increase greater than 2.5. Considering the total population of the Lisbon Metropolitan Area, $16 \%$ of the daytime population is potentially exposed, compared to $8 \%$ of the resident population during the nighttime period. While population exposure to the moderate hazard level remains relatively stable at $3 \%$, a considerable 
Advancing tsunami risk assessment by improving spatio-temporal population exposure and evacuation modeling

increase from $5 \%$ during nighttime to $12 \%$ during daytime is observed in the highly susceptible areas. These results reflect the location of human economic activities closer to the coastline and highlight the more intensive occupation of these areas during the daytime period.

Table 13 Population exposed to Tsunami inundation levels in nighttime and daytime periods in the LMA

\begin{tabular}{|c|c|c|c|}
\hline Tsunami hazard & \multicolumn{2}{|c|}{ Population } & \\
\hline [Inundation levels] & $\begin{array}{c}\text { Abs. } \\
\text { [Pers.] }\end{array}$ & $\begin{array}{l}\text { Rel. } \\
{[\%]}\end{array}$ & \\
\hline High & 125,730 & 59 & \\
\hline Moderate & 86,929 & 41 & $\frac{2}{6}$ \\
\hline Total & 212,659 & 100 & $\overrightarrow{+}$ \\
\hline High & 334,000 & 78 & \\
\hline Moderate & 93,444 & 22 & ס्ָ \\
\hline Total & 427,444 & 100 & \\
\hline High & 208,270 & 166 & च \\
\hline Moderate & 6,515 & 7 & हैं \\
\hline Total & 214,785 & 101 & \\
\hline
\end{tabular}

*Relative differences are relative to the night

numbers

Regarding the modeling of tsunami evacuation, Figure 20 shows the modeled surfaces of evacuation time and access points to safe areas (i.e., outside of the inundation zone), for the nighttime (A) and daytime (B) periods, respectively. The differences for nighttime and daytime evacuation times become intensified by the fact that densely populated areas are slower to evacuate, as a higher 'traffic volume' of evacuees slows down the speed of every individual (Klupfel 2005; Rogsch 2005). This is also considered in the modeling process. Therefore, longer evacuation periods are observed in daytime, since about 40,000 people are estimated to be present in the hazard zone in the three communes in this period, compared to 8,000 residents in nighttime. 

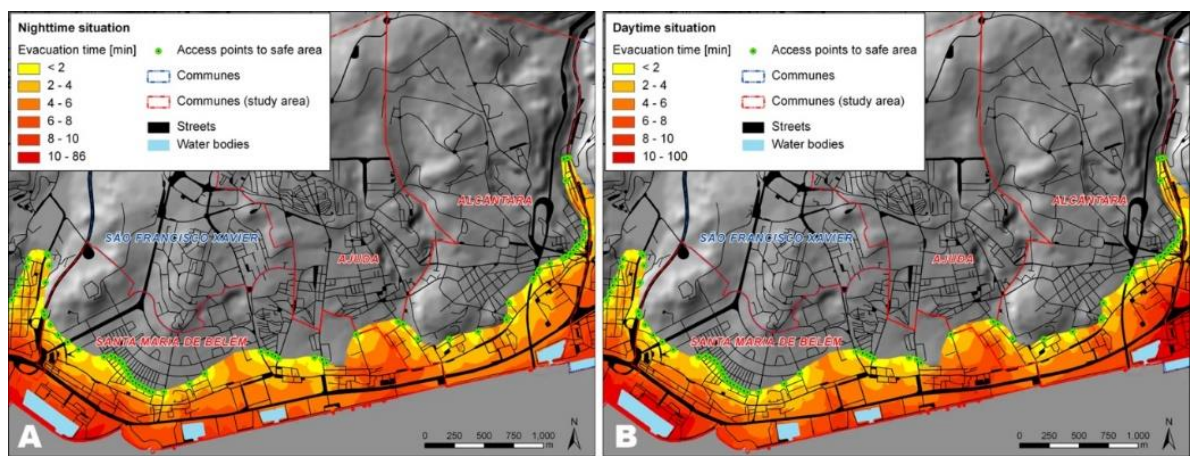

Figure 20 Nighttime $(A)$ and daytime (B) Tsunami evacuation modeling

Results of the evacuation modeling analysis reveal significant differences in the magnitude and speed of evacuation between night and day (Table 14). Differences are especially striking in the commune of Alcantara, due to the configuration of hazard zone and population distribution. Overall in the study area, all resident (nighttime) population is able to evacuate to safety within 10 min. In daytime, although more than $95 \%$ of the population has reached safety after $20 \mathrm{~min}$, it takes as long as $90 \mathrm{~min}$ for a full evacuation. This duration is longer than the travel time to this location for the devastating 1755 tsunami (approx. 50-70 min; Baptista et al. 1998), implying that for a similar scenario, there could be approximately 700 people in the study area who would not reach safe areas on foot, according to the modeled results. This is assuming that everyone present in the hazard zone would initiate evacuation to closest safe areas immediately following a large earthquake (greater than $M=6.5$ ) or tsunami warning, but no early-warning and evacuation system for tsunamis is yet implemented in Lisbon. Therefore, it would be important to consider other safety measures, such as vertical evacuation, that would shorten travel time to safety. Also, this analysis would be improved by further considering nonresidents and nonstudents/workers (e.g., tourists) present and involved in leisure activities, which are especially relevant in the Alcantara (nightlife) and in the Belem (daytime museums and monuments) communes. For this exercise, we are not considering the specific effects in the area that a triggering earthquake might cause in people, buildings and other structures, impacting the ensuing evacuation from tsunami, since such a detailed and spatially explicit modeling is not available.

Table 14 Population remaining in hazard zone and successful evacuees after different time intervals of tsunami evacuation

\begin{tabular}{|c|c|c|c|}
\hline \multirow[t]{2}{*}{ Commune } & \multirow[t]{2}{*}{$\begin{array}{c}\text { Elapsed } \\
\text { time }\end{array}$} & \multicolumn{2}{|c|}{ Population } \\
\hline & & Remaining & Evacuees \\
\hline
\end{tabular}


Advancing tsunami risk assessment by improving spatio-temporal population exposure and evacuation modeling

\begin{tabular}{|c|c|c|c|c|}
\hline Santa & 0 & 4,300 & $\overline{0}$ & \\
\hline Maria de & 5 & 1,600 & 2,700 & $\underline{0}$ \\
\hline Belém & 10 & 0 & 4,300 & \\
\hline \multirow{6}{*}{$\begin{array}{c}\text { Santa } \\
\text { Maria de } \\
\text { Belém }\end{array}$} & 0 & 25,800 & 0 & \multirow{6}{*}{ ஜू } \\
\hline & 5 & 19,900 & 5,900 & \\
\hline & 10 & 4,600 & 21,200 & \\
\hline & 15 & 500 & 25,300 & \\
\hline & 20 & 400 & 25,400 & \\
\hline & 75 & 0 & 25,800 & \\
\hline \multirow{3}{*}{ Alcântara } & 0 & 3,800 & $\overline{0}$ & \multirow{3}{*}{$\frac{2}{\frac{0}{7}}$} \\
\hline & 5 & 300 & 3,500 & \\
\hline & 10 & 0 & 3,800 & \\
\hline \multirow{6}{*}{ Alcântara } & 0 & 17,700 & 0 & \multirow{6}{*}{ ֻัֶ } \\
\hline & 5 & 9,200 & 8,500 & \\
\hline & 10 & 900 & 16,800 & \\
\hline & 15 & 200 & 17,500 & \\
\hline & 20 & 200 & 17,500 & \\
\hline & 90 & 0 & 17,700 & \\
\hline \multirow{2}{*}{ Ajuda } & 0 & 200 & $\overline{0}$ & \multirow{2}{*}{$\frac{z}{2}$} \\
\hline & 5 & 0 & 200 & \\
\hline \multirow{2}{*}{ Ajuda } & 0 & 200 & 0 & \multirow{2}{*}{ ஜू } \\
\hline & 5 & 0 & 200 & \\
\hline
\end{tabular}

\subsection{Conclusions and outlook}

This work is an initial approach toward considering the spatio-temporal population distribution to assess risk of tsunami in a large metropolitan area. The region is prone to this hazard and was severely affected by this type of event in the past. Improved high-resolution data sets of nighttime and daytime population density were developed and combined with existing Tsunami Inundation Susceptibility zones to estimate potential population exposure in those periods in the Lisbon Metropolitan Area. In addition, evacuation speed was modeled and analyzed for both scenarios in a subset of the study area. Results show that potential population exposure to tsunami is significantly time-dependent, displaying a strong variation from nighttime to daytime. The 
analysis indicates that a significant amount of population is at risk, and its numbers increase dramatically from nighttime to daytime, especially in the zones of high susceptibility (i.e., lower elevation and closer to the coastline). Evacuation modeling reveals that full horizontal evacuation may be problematic in the daytime period, even if initiated immediately after an early warning or a major tsunami-triggering earthquake event. This finding recommends that additional measures be considered as alternatives, such as vertical evacuation.

As with any similar exercise, this model implies a number of uncertainties that can affect the specific results of population distribution, exposure and evacuation. However, the analyses provide valuable information for further development of scenarios leading to evacuation planning and management measures, yet to be implemented in the study area. This improved characterization of vulnerability and risk can benefit all phases of the disaster management process where human exposure should be considered, namely in emergency planning, risk mitigation, preparedness and response to an event. Considering the large variations between nighttime and daytime population exposure that become apparent in the presented case study model, it is clear that integration of this kind of information in a pre-event assessment stage would favor preparation for actual hazardous events and thus be a huge step toward improved mitigation and eventually saving lives. The new population surfaces can also be used as input in loss simulators for modeling of human casualties resulting from different types of hazards and threats, such as earthquakes, flash floods, landslides or chemical accidents. Given the availability of input data sets, this approach could, for example, also be applied to the Oporto Metropolitan Area, further encompassing nine municipalities and $1,260,680$ inhabitants.

Planned future developments include conducting a more detailed spatial modeling of population distribution and exposure assessment to tsunami at the building level considering its function, height and elevation (Aubrecht et al. 2010). This will also enable modeling of vertical evacuation, that is, evacuation to a safe structure or floor above the inundation level. Subsequent analyses would also benefit from assessing likely population exposure in additional temporal cycles, relevant for population dynamics in large urban centers (e.g., rush hour, week-ends, etc.). Especially useful for tsunami risk assessment would be to account for seasonal differences, given that human occupation of beaches is usually very high in warm summer days.

Acknowledgments:

The authors thank Prof. J. L. Zezere and the RISKam group (CEG-IGOT) for providing the tsunami inundation susceptibility map. 
Advancing tsunami risk assessment by improving spatio-temporal population exposure and evacuation modeling 


\section{Chapter 5. Enhanced data and methods for improving open and free global population grids: putting 'leaving no one behind' into practice ${ }^{37}$}

37 This chapter is published as:

Freire S., M. Schiavina, A. J. Florczyk, K. MacManus, M. Pesaresi, C. Corbane, O. Borkovska, J. Mills, L. Pistolesi, J. Squires \& R. Sliuzas, 2018. Enhanced data and methods for improving open and free global population grids: putting 'leaving no one behind' into practice. International Journal of Digital Earth, 13:1. DOI: $10.1080 / 17538947.2018 .1548656$ 
Enhanced data and methods for improving open and free global population grids: putting 'leaving no one behind' into practice

\begin{abstract}
Data on global population distribution are a strategic resource currently in high demand in an age of new Development Agendas that call for universal inclusiveness of people. However, quality, detail, and age of census data varies significantly by country and suffers from shortcomings that propagate to derived population grids and their applications. In this work, the improved capabilities of recent remote sensing-derived global settlement data to detect and mitigate major discrepancies with census data is explored. Open layers mapping builtup presence were used to revise census units deemed as 'unpopulated' and to harmonize population distribution along coastlines. Automated procedures to detect and mitigate these anomalies, while minimizing changes to census geometry, preserving the regional distribution of population, and the overall counts were developed, tested, and applied. The two procedures employed for the detection of deficiencies in global census data obtained high rates of true positives, after verification and validation. Results also show that the targeted anomalies were significantly mitigated and are encouraging for further uses of free and open geospatial data derived from remote sensing in complementing and improving conventional sources of fundamental population statistics.
\end{abstract}

\title{
5.1 Introduction
}

Accurate geospatial data on global population distribution and characteristics are increasingly required and relied upon for analysis and modelling in an expanding range of disciplines (Gaughan et al. 2014; Wardrop et al. 2018). These data are also crucial in the frame of the Digital Earth perspective in the path towards decision-making (Shupeng and van Genderen 2008). In the context of evidence-based assessment for policy support, the recent wave of post-2015 international development agreements (including the Sendai Framework for Disaster Risk Reduction 2015-2030 (adopted March 2015); United Nations (UN) 2030 Agenda for Sustainable Development (SDGs, September 2015); COP 21 Paris Agreement on Climate Change (November 2016); UN New Urban Agenda (December 2016)) places great demands and responsibility on geospatial data, and in particular on that related to population.

Public information about the characteristics and spatial distribution of population is a strategic resource supporting the monitoring and implementation of international frameworks. Information about population density per uniform spatial sampling schemas (e.g. grid cells) are necessary for modelling critical aspects of human-environment interaction, such as: Exposure (to hazards, pollutants); Access (to resources, services, facilities); and Impacts, in both perspectives of (i) the impact of human activities on the planet and (ii) impact of the environment on the people living on the Earth's surface (natural disasters, environmental change) (UNISDR 2015a; UNDESA 
2016; UNECOSOC 2016). The Sustainable Development Goals (SDG) indicators are 'action oriented, global in nature and universally applicable' and should be themselves sustainable and comparable across space and time (UNECOSOC $2016,7)$. Ideally, the indicators supporting the international development agenda should be based on geospatial population data that is up-to-date, sufficiently detailed, accurate, consistent, cost-effective (i.e. sustainable), transparent (i.e. using clear methods), and accessible to all. These development agreements, their targets and monitoring needs (i.e. indicators) present an opportunity not only for producing more data, but also for improving existing datasets. The reliability of international statistics is periodically called into question (e.g. Wolff, Chong, and Auffhammer 2011), and the recent surge in initiatives aimed at producing more accurate and detailed statistics (e.g. by UN (UNDP 2018), World Bank (WB 2018), Bill \& Melinda Gates Foundation and UK Department of International Development) are a realization that statistical capacity must be strengthened (UNECOSOC 2016).

Regular grids (raster or vector) are now well established and widely used spatial structures to model and report population attributes, and their advantages are largely recognized (Deichmann, Balk, and Yetman 2001). Several global gridded population datasets are currently produced. They are differentiated by the underlying population concept (e.g. 'resident' vs. 'ambient' population), gridding method, and distribution policy (for reviews and recent developments see Linard and Tatem 2012; Stevens et al. 2015; Freire et al. 2016a). For a given spatial unit, population distribution grids can be produced by disaggregating (e.g. gridding) population counts (top-down approach) or by estimating that count at the grid cell level through combining sampling with ancillary data (bottom-up method) (Wardrop et al. 2018). Global population grids aiming to support policies and international agreements in global forums are typically based on available statistics whose national totals match, or are adjusted to match, those used in UN population estimates (e.g. UNDESA 2015). UN estimates are used as a standard in order to produce more accurate and harmonized datasets for countries where census data are considered to be less reliable, and because of the extensive work done by the UN Population Division to adjust and correct census data post enumeration. Examples of such grids include Gridded Population of the World (GPW) (Deichmann, Balk, and Yetman 2001; Doxsey-Whitfield et al. 2015) the Global Rural-Urban Mapping Project (GRUMP) (Balk et al. 2006), and the Global Human Settlement Population (GHS-POP) (Freire et al. 2016a). For population grids that are generated by disaggregating available statistics (top-down) while preserving their volume, output accuracy (with respect to ground truth data) is largely dependent on the spatial detail and quality of input geospatial layers. In addition to their spatial detail, population census-like layers vary widely in quality concerning their geometry, attributes, reliability, and currency. Incidentally and unfortunately, the quality and reliability of statistics' are 
Enhanced data and methods for improving open and free global population grids: putting 'leaving no one behind' into practice

particularly low in many developing countries (Tatem et al. 2007), at whom the post-2015 development agenda is especially directed.

Production of global grids relies on population sources that are somewhat heterogeneous in respect to these characteristics. These sources include provisional or final censuses originating in National Statistical Offices (NSOs) as well as estimates and projections provided by Non-governmental (NGOs) and other organizations. Despite valuable efforts to collect, integrate, and improve global census data obtained from disparate sources (e.g. GPW), some important requirements remain unsolved. Addressing the universal inclusiveness requisite associated with the UN 'leaving no one behind' imperative of the new sustainable development agenda (UNDESA 2016) requires that all people are counted and accounted for in the place they live. However, census enumerations often do not meet this requirement (even sometimes by design as in de facto census), and any statistical sampling inherently lacks information about marginal and uncounted populations.

Perhaps surprising and unknown to some users, available population statistics also suffer from error and uncertainty that affect even the most basic of demographic variables (i.e. total population). While uncertainty is difficult to assess and communicate, error often propagates unimpeded to downstream analyses and applications. The main deficiencies and shortcomings affecting geospatial population statistics can be summarized in the following basic taxonomy:

1. Issues affecting geography (census reporting units): a) Lack of spatial detail (i.e. too coarse or generalized, related to spatial precision) b) Low spatial accuracy (i.e. units partially displaced or completely misplaced)

2. Issues affecting attributes (population counts in present case) a) Undercounting and/or underreporting (i.e. underenumeration) b) Overcounting and/or over-reporting (i.e. overenumeration)

These issues have been unnoticed or unaddressed due to the lack of external accuracy assessment related to a shortage in independent compatible highresolution reference data. Notable exceptions are the works of Hay et al. (2005) and Mondal and Tatem (2012) that discuss deficiencies in the representation of population distribution along coastlines, and Linard and Tatem (2012) that address these in frame of infectious disease research. In contrast to remote sensing-based maps where validation is expected to rely on independent reference data, typically collected from ground truthing (see Congalton and Green 2009), quality assessment of population grids is usually limited to internal consistency of model performance (i.e. population data used for validation is the same that was used for modelling, just finer). Also, the relative coarse resolution of global grids produced until recently (i.e. $\sim 1-5 \mathrm{~km}$ ) mitigated the impact of some deficiencies that now become apparent as cell sizes approach $\sim 100 \mathrm{~m}$. 
Significant improvements to the quality of population grids are to be gained by addressing some of these shortcomings. Detection and mitigation of deficiencies in population statistics require independent, reliable, higherresolution data. Remote sensing imagery and methods have been evolving towards constituting a more detailed, objective and independent data source on human presence on the Earth surface. The combination of new costeffective, automated and fully replicable data classification methods (e.g. machine learning) with the synoptic capacities of satellite Earth Observation imagery, made accessible in a public, full open-and-free frame, can contribute to fill information gaps and supplement existing statistics by mitigating some major shortcomings in population data. This is especially true in poor, remote, unsafe, disputed, very large, and/or highly dynamic areas of the globe where conventional data gathering and updating is challenging.

Current methods and processing capacity allow for global mapping of built-up areas and settlements with unprecedented spatio-temporal detail and accuracy - in essence capturing the local scale with global coverage, finally starting to fulfil a long-standing promise of remote sensing technology. Making these datasets available open and free helps to increase access, promotes transparency, and ensures accountability of the information produced. Global, consistent and updated geospatial data such as that made openly available in the framework of the Global Human Settlement Layer (GHSL) (Pesaresi and Ehrlich 2009) are already providing an effective contribution and improving the disaggregation of census data into derived population grids (Freire et al. 2015d; Linard et al. 2017; Nieves et al. 2017). However, it remains to be tested in large scale if such remotely sensed data can also assist in assessing and mitigating major deficiencies present in geospatial population statistics.

This article addresses shortcomings present in global collections of census data, by introducing, applying, and discussing novel procedures aimed at investigating and detecting some of those major anomalies. It then demonstrates mitigation of some inconsistencies using high-resolution geospatial data derived from remote sensing. The focus is on deficiencies in mapping coastlines and declared unpopulated areas, as extreme instances of deficiencies 1a) and 1b) and 2a) in the taxonomy mentioned above. Making use of open and free high-resolution global settlement layers derived from contemporary satellite imagery, the approaches are illustrated with GHSL in the frame of producing GHSL-based new global population grids (release 2018).

\subsection{Materials and methods}

Two main types of geospatial data were used in this work: vector-based census data reporting on estimated total population counts for the target year of 2015 (http://sedac.ciesin.columbia.edu/gpw), and raster layers reporting on builtup presence for 2013/2014 derived from Landsat image collections in the 
Enhanced data and methods for improving open and free global population grids: putting 'leaving no one behind' into practice

frame of the GHSL project (http://ghslsys.jrc.ec.europa.eu/index.php). Some aspects of these two datasets are compared and combined, with remote sensing data being used to identify anomalies in the geospatial census data. These anomalies are mitigated with the support of the remote sensing-derived data after verification and validation.

\subsubsection{Data sets}

\subsubsection{Geospatial data on census population}

As a source of census population data, a database assembled by the Center for International Earth Science Information Network (CIESIN) in the frame of the Gridded Population of the World project (Tobler et al. 1997) was used. For more than two decades, GPW has been collecting, combining, and harmonizing available population census and estimates into what is considered the most complete, detailed and coherent census-based geodatabase available globally. This database is periodically updated with more recent and improved data, with GPW employing clear and transparent methods to create open and free residence-based population grids for different reference years (DoxseyWhitfield et al. 2015). Despite these efforts, the GPW databases are subject to availability and quality of source (national) population statistics, and therefore inherit their gaps and shortcomings, as reliability and currency of population data is quite heterogeneous among countries (for GPW metadata see CIESIN 2017a).

The GPW data used consisted in country-based layers (one for each of 241 countries) of census and administrative polygons containing estimated residential population for the GHSL target years 1975-1990-2000-2015, adjusted to country-level estimates of UN World Population Prospects 2015 (UNDESA 2015). Due to the development of the work and the updating schedule of GPW data, two versions from the same release were used: GPWv4, released in 2016 (CIESIN 2016), was used for detection of inconsistencies along coastlines; GPWv4.10, available in late 2017 (CIESIN 2017b), was used for revision of unpopulated areas. GPWv4.10 is a revision of GPWv4 with boundary or population updates for 64 countries. More details about these data can be found here: http:// sedac.ciesin.columbia.edu/data/collection/gpw-v4.

\subsubsection{Built-up areas from remote sensing}

In the frame of the Global Human Settlement Layer (GHSL) project, global built-up (BU) areas were recently mapped with unprecedented spatial detail, consistency, and temporal coverage (Pesaresi et al. 2016). The GHSL has developed and employed novel and automated approaches to produce a time series of raster layers reporting on the presence of building structures, defined as 'all constructions above ground intended for human or animal sheltering or for the production of economic goods' (Pesaresi et al. 2013). These data were derived from Landsat image collections spanning four periods: 1975, 1990, 
2000 and 2013-2014 and are made available open and free. Quality assurance was conducted to validate these data against a heterogeneous set of available layers mapping building footprints (Pesaresi et al. 2016). More recently, independent spatio-temporal quality assessment of the GHSL built-up time series was performed for the USA showing very encouraging accuracy that generally increases over time (Leyk et al. 2018). Most relevant for the present work, these built-up areas exhibited strong correlation with population distribution and density, and suitability for population disaggregation and modelling (Freire et al. 2015d, 2016; Linard et al. 2017; Nieves et al. 2017).

In this effort, global layers mapping built-up areas for the latest epoch (20132014), at spatial resolutions of 38 and $250 \mathrm{~m}$, were used as an indication of the presence of human settlements. Due to the development timeline of this work and the updating schedule of GHSL products, data from different releases were used: the GHS-BUILT data released in 2015 (GHS_BUILT_LDSMT_GLOBE_R2015B) were used for combination with census data from GPW for initial flagging and systematic detection of discrepancies (Pesaresi et al. 2015a); while the latest and improved GHSBUILT data (GHS_BUILT_LDSMT_GLOBE_R2018A), produced in Fall 2017 (Corbane et al. 2017), to be publicly released in Fall 2018, were used for population disaggregation and creation of final population grids. All geospatial layers were projected to World Mollweide projection (EPSG 54009), the equal-area projection adopted for production of the GHSL global population grids. This is also important for the processing undertaken and described here, which is mostly based in quantification and comparison of surfaces.

\subsubsection{Revision of 'unpopulated' units}

An extreme case of issue $2 a$ ) of the taxonomy of census anomalies proposed in Section 1. is the reporting of areas as 'unpopulated' or 'uninhabited' or otherwise as containing no population. During the early stages of production of GHSL global population grids, it was observed that some units declared as having no population in census data in fact contained settlements and significant areas of built-up according to the GHS-BUILT data. Consequently a spatial analysis procedure was designed to (i) check for the presence of (resident) population in census units where no population was reported, and (ii) mitigate these issues while minimizing changes to the input census data (conservative approach). Units deemed as 'uninhabited' or otherwise unpopulated in the census data were critically assessed for the presence of significant residential population, based on ancillary data (e.g. coordinates of populated places) and very high resolution (VHR) imagery. Such census units were then selected based on their size and the extent of built-up surface in 2014 as an indication of the likely presence of settlements. Due to the experimental nature of the work, a sequential approach was adopted in order to inspect, control, and calibrate the assumptions made at each step. Adopting 
a conservative approach was important to quickly narrow the selection to the main issues and limit the rate of false positives. For selection of potentially problematic census units, a simple semi-heuristic rule-based approach was followed. From the set of census units in GPWv4.10 having no population $(238,029$ units), most non-residential zones were filtered out by selecting polygons larger than $300 \mathrm{ha}(3 \mathrm{~km} 2)$. This threshold was adopted because it is the mean size of non-residential built-up patches (i.e. industrial, commercial, and service facilities) in the CORINE Land Cover (CLC) 2012 vector map, v18.5.1 (EEA 2016). CLC2012 currently covers 39 countries and $5.8 \mathrm{M} \mathrm{km} 2$ in Europe, and this threshold may not be the best one to use in other contexts.

From these 47,508 census units larger than 300 ha, units were selected that contained a total surface of built-up in 2014 (according to GHS-BUILT R2015B) greater than 10 ha, to increase confidence in presence of significant resident populations. As the resulting set of 2,717 units was still too large a number for visual inspection with available resources, and after confirming it would include a high rate of false positives in countries that have both detailed and accurate censuses, countries deemed, based on our in-depth empirical knowledge of the data, as having reliable census ( 14 of the 45 countries in set) were excluded. This led to the identification of potentially problematic census units in 31 countries, to be visually inspected for verification and validation of the approach. These units were inspected using VHR imagery from web mapping services (Esri World Imagery service through the ArcMap application). After collecting sufficient visual evidence that some units were inhabited (i.e. presence of residential-type buildings with signs of habitation), that anomaly had to be mitigated. A decision was taken to adopt the most conservative approach that would imply minimal changes to the GPW's geospatial census dataset and still ensure that some population would be accounted in those areas, to be then disaggregated to the mapped BU areas in the creation of GHS-POP grids. To implement this, a 'smart' geoprocessing approach was devised and automated (hereafter called 'split and merge') to assign population to those previously unpopulated areas without altering the boundaries and the total population of the upper administrative level. The approach involves splitting and merging the confirmed problematic polygons, based on geographical proximity to those ones adjacent and containing population, and merging the split parts to the latter.

Figure 21 shows the main steps involved in the application of the 'split and merge' approach. This method consists in spatially dividing the problematic polygons according to a proximity rule accounting for adjacent populated polygons belonging to the same administrative unit. This task is performed by (i) re-projecting the polygons to an equidistant projection, more suitable for measuring distances (i.e. World Equidistant Cylindrical), (ii) rasterizing to a sufficiently fine resolution (i.e. a trade-off between accuracy and computational demand) with an all-touched approach, (iii) applying a nearest-neighbour 
nibbling technique (only considering populated neighbouring units), (iv) polygonising the resulting part and finally $(\mathrm{v})$ re-projecting back to the original projection. The polygons obtained are then cleaned of geometric artefacts by clipping them to the original boundary. Each generated part of the problematic polygon is then dissolved with the related populated neighbouring unit, in this way 'populating' the whole original problematic area.

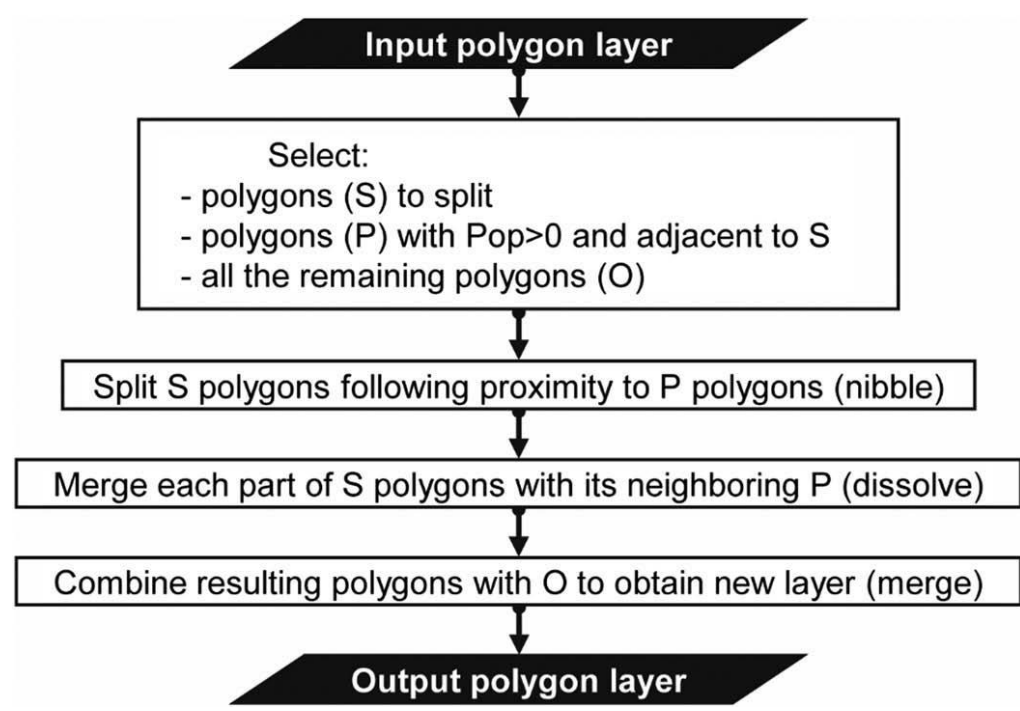

Figure 21 Flow chart of the 'split and merge' approach

\subsubsection{Harmonization of population and settlement data along coastlines}

Seashores and waterfronts can be especially intense and dynamic zones, due both to natural processes and their attractiveness for settlement and building of infrastructure (EEA 2006; McGranahan, Balk, and Anderson 2007; Freire, Santos, and Tenedório 2009). These strong and fast dynamics contribute to making census or administrative geometries outdated or inaccurate. Moreover, the geospatial census data are not produced with globally harmonized technical specifications, thus including different nominal scales and spatial tolerance characteristics. The characteristics of the GHSL layer mapping built-up areas (i.e. seamless coverage, uniform technical specs, decametric-scale spatial detail, currency) have revealed discrepancies with GPW along coastlines (including inland water bodies). Most of these consisted in the coastline from GPW being inland respect to that actually detected by GHSL, implying in practice that no population would be disaggregated to those mapped built-up areas in GHSL that were water bodies in GPW (i.e. spatial mismatch between source and target zone). This would cause changes to the population density 
and extent of coastal settlements or omit them entirely in the resulting population grids. Therefore, a systematic procedure was developed aiming at identifying globally the main inconsistencies between the two datasets along coastlines, and efforts were undertaken to reconcile them. This procedure was aimed at harmonizing population and settlement data along coastlines, but does not intend to harmonize the delineation of water bodies themselves. The high-resolution (38 m) GHSL layer on built-up areas for 2014 (GHS-BUILT from R2015B), available at the time of work, was used to detect the significant human presence (i.e. BU) beyond GPWv4 censuses coastlines. The process of detecting inconsistencies included the following three main steps: (i) creation of a layer depicting the built-up areas outside of the GPW landmass, (ii) identification of potentially inhabited patches containing built-up, and (iii) visual inspection and validation of the patches. It is well known that full automatic detection of built-up areas from satellite imagery is prone to commission errors in sandy or rocky landscapes, and, as a result, part of the builtup area pixels in coastal areas might be false positives. Therefore, a visual inspection was required. It should be noted that the term coastline here refers to inland water bodies as well as seashores, and the level of detail at which coasts are outlined varies widely across countries (e.g. the inland water bodies in the USA are depicted with high detail while in most other countries only the large ones are outlined with low detail).

During step (i), first a water mask derived from the country polygons of GPWv4 data was rasterized at approximately $38 \mathrm{~m}$, and only within the data domain of the GHSL layer as defined by the GHSL Datamask product (Pesaresi et al. 2015b). Then, this coastal water layer was intersected with the GHS-BUILT layer, producing the built-up areas not overlapping with any GPW country polygons. In total, these areas have amounted to $6,142 \mathrm{~km} 2$ of surface. The procedure for selecting and validating potentially inconsistent patches included three steps: (1) vectorizing connected cells classified as built-up surface (using 4-connectivity rule) into individual polygons (nearly 800,000 patches, with a mean surface of $7700 \mathrm{~m} 2$, corresponding to $5 / 6$ contiguous pixels), (2) selecting all patches larger than $1 \mathrm{~km} 2$ (287 selected), and (3) visual inspection using GIS software and very high resolution (VHR) satellite imagery from web mapping service (Google Maps) to confirm presence of buildings. The $1 \mathrm{~km} 2$ size threshold was chosen to balance importance and potential impact of inconsistencies with resources available for verification and validation. Figure 22 shows examples of built-up patches located beyond the coastline of GPW in Japan, Tunisia, China, and Romania, assessed during visual inspection. For the selected patches (those larger than $1 \mathrm{~km} 2$ ) in which visual inspection confirmed presence of built-up surfaces, mitigation consisted in reconciling the outline of GPW census units with built-up patches by manually extending the units on the coast to include these areas. 

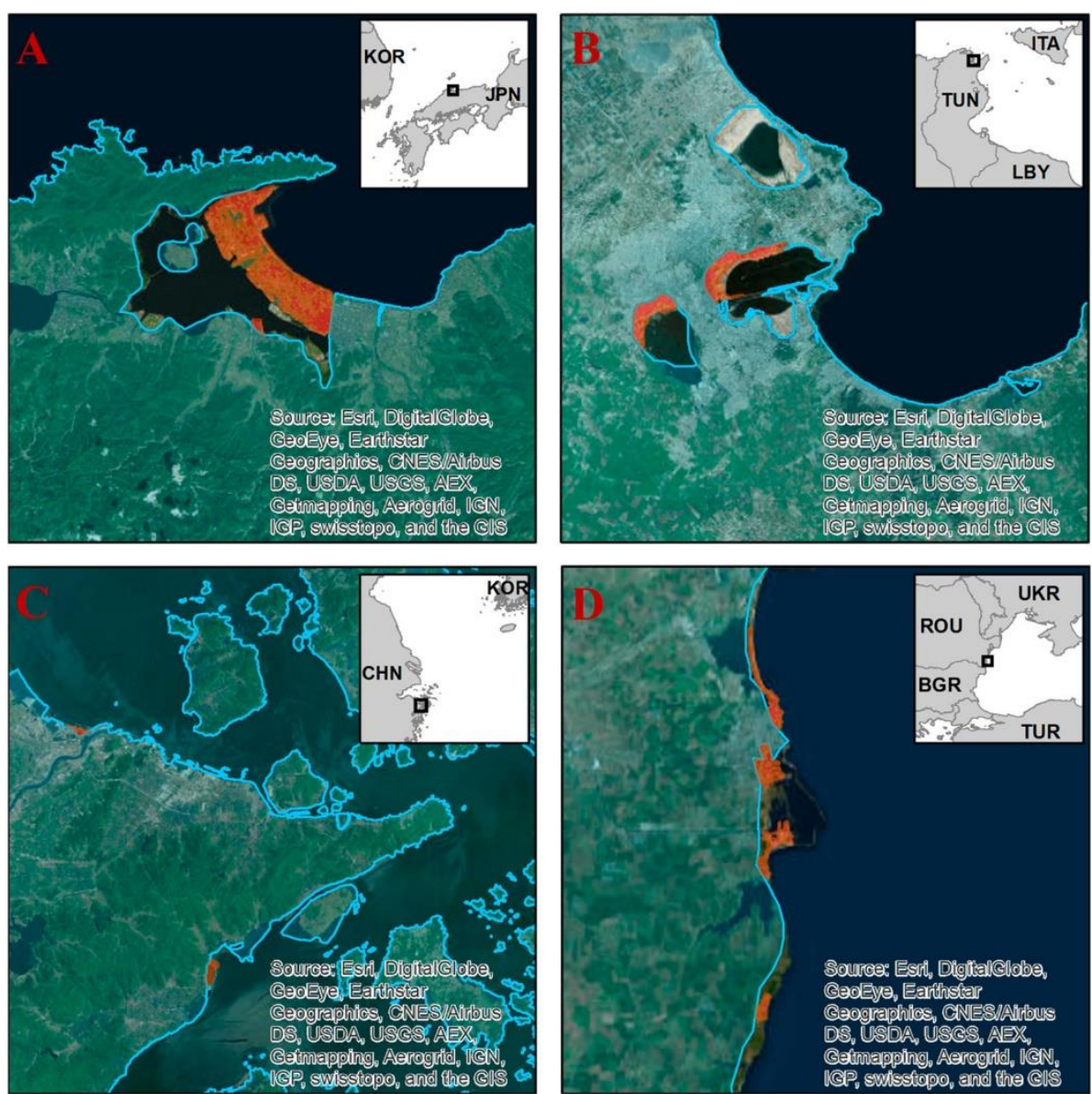

Figure 22 Examples of detected discrepancies (patches larger than $1 \mathrm{~km}^{2}$ ) between GPWV4 (blue line) and GHS-BUILT 2014 (orange) along the coasts of (A) Japan (JPN), (B) Tunisia (TUN), (C) China (CHN), and (D) Romania (ROU)

After conducting automatic detection and during visual inspection of the main coastal discrepancies between GPWv4 and GHSL, systematic or additional inconsistences were identified. These systematic inconsistencies between the more recent and improved GHS-BUILT P2018 and GPW4.10 layers were still present in some countries: Japan, Ukraine, Switzerland, and France. Therefore, an automated approach to mitigate these issues that appear to be caused by the coarser scale (low spatial detail) and/or positional errors (poor georeferencing) of census reporting units was developed and applied. For inconsistences detected in lakes (i.e. Switzerland and France) the 'split and merge' approach (described in Section 2.1) was directly applied to polygons representing lakes in order to split them and dissolve each part with the nearest neighbour polygon. In coastal areas, such as in Ukraine and Japan, the GPW coastline was first extended seawards using a 2-km external buffer. Next, 
Enhanced data and methods for improving open and free global population grids: putting 'leaving no one behind' into practice

this buffer was split according to proximity to the original census units by using the split and merge approach, and then assigned each part of the buffer to a census polygon (see Figure 28). In this case, the whole set of census polygons was used, without selecting only the populated areas.

\subsection{Results and discussion}

\subsubsection{Revision of unpopulated units}

The implementation of the semi-automated rule-based procedure to the initial 238,029 census units deemed as uninhabited in GPW geospatial census data resulted in the selection of 128 units in 31 countries as potentially containing significant resident population (Figure 23).

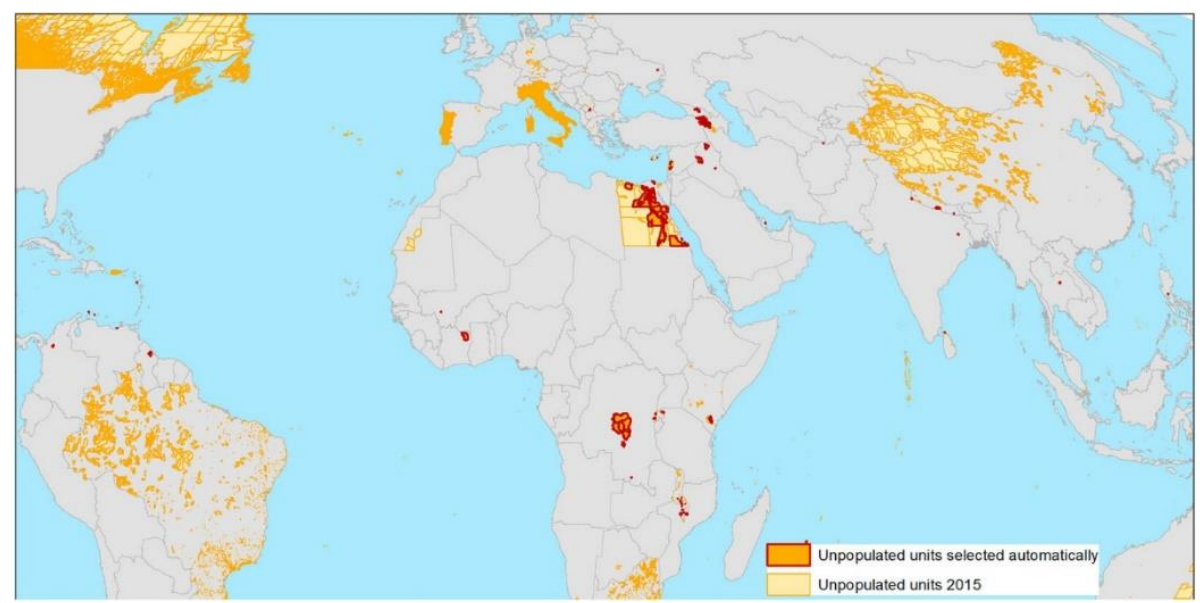

Figure 23 Global distribution of census units without population in GPWV4.10 data and those flagged based on the automated procedure developed (USA not considered). Note that existence of unpopulated units is merely a feature of census design and not of the landscape

All of these 128 units were validated visually with VHR imagery from web mapping services (Esri/Bing, Google maps). This was both to proceed with confidence in such a sensitive issue, and to assess the validity of the semiheuristic rule-based approach used for selection of problematic units. The validation with VHR imagery revealed the presence of significant residential areas having clear signs of habitation in 76 census units, belonging to 19 countries (Table 15), resulting in a success rate of the automated procedure of around 59\% (of polygons). These 76 problematic units have a combined area of $297,090 \mathrm{~km} 2$, of which $624 \mathrm{~km} 2$ are reported by GHSL to be covered by building structures in 2014. If this built-up surface is accurate and all of it is dedicated to residential function, using a low occupancy rate of $100 \mathrm{~m} 2$ of builtup surface per person would put a conservative estimate of under-reported 
population in these units at more than 6 million people. Some recent estimates put the value of 'missing' (i.e. undercounted) people in urban slums at 369 million worldwide (Carr-Hill 2017).

Table 15 List of countries in which problematic polygons were selected

\begin{tabular}{lccr}
\hline Country & $\mathbf{N}$ & $\begin{array}{c}\text { Area } \\
\mathbf{( k m}^{\mathbf{2}} \mathbf{)}\end{array}$ & $\begin{array}{r}\text { BU area } \mathbf{2 0 1 4} \\
\left(\mathbf{k m}^{\mathbf{2}} \mathbf{)}\right.\end{array}$ \\
\hline Afghanistan & 1 & 76.7 & 0.1 \\
Armenia & 1 & 53.2 & 2.0 \\
Democratic Republic of & 7 & $99,575.9$ & 154.9 \\
the Congo & 1 & 514.1 & 0.1 \\
Colombia & 1 & 4.4 & 0.4 \\
Cyprus & 16 & $184,254.7$ & 283.2 \\
Egypt & 2 & 20005.2 & 1.8 \\
Georgia & 1 & 918.6 & 0.7 \\
Guyana & 1 & 116.4 & 70.3 \\
India & 4 & 8459.9 & 65.7 \\
Iraq & 4 & 56.1 & 0.7 \\
Lebanon & 2 & 53.5 & 14.0 \\
Mali & 14 & 121.1 & 4.7 \\
Malawi & 1 & 163.9 & 1.7 \\
Nepal & 1 & 86.6 & 0.9 \\
Philippines & 1 & 37.7 & 0.2 \\
Rwanda & 16 & 214.5 & 7.5 \\
Serbia & 1 & 306.4 & 2.1 \\
Thailand & 1 & 71.1 & 12.6 \\
Ukraine & 76 & 297,090 & 623.7 \\
\hline Total & Note: For each country the number of polygons (N), the sum of the area of \\
such polygons and the total built-up (BU) surface accounted by GHSL built- \\
up layer in 2014 is reported.
\end{tabular}

Mitigation of these anomalies was carried out using the 'split and merge' approach, which was applied to a total of 58 units covering about 296,670 $\mathrm{km} 2$, in the following countries: Afghanistan, Armenia, Democratic Republic of the Congo (DRC), Colombia, Cyprus, Egypt, Georgia, Guyana, Iraq, Lebanon, Mali, Malawi, Nepal, Rwanda, Thailand, and Ukraine. Among them, the Democratic Republic of the Congo and Egypt are the most problematic, with the surface of incorrectly labelled 'unpopulated' units reaching $99,576 \mathrm{~km} 2$ and $184,255 \mathrm{~km} 2$, respectively. In Egypt some problems seem to be caused by the coarse spatial detail and spatial inaccuracy of census polygons.

Figure 24 shows the results of the application of the 'split and merge' approach in Guyana and Lebanon. In Guyana, the declared unpopulated polygon (Figure 24 (A1)) is split into seven parts and is then dissolved with adjacent polygons belonging to the same administrative level (Figure $24(\mathrm{~A} 2)$ ). In Lebanon the setting is more complex: the target polygon (Figure $24(B 1)$ ) is split into four parts which are then dissolved with the neighbouring administrative units, 
Enhanced data and methods for improving open and free global population grids: putting 'leaving no one behind' into practice

three of which are to the East of a unit (in grey) correctly declared as uninhabited. The resulting polygons, preserving their respective population counts, replace the problematic one in the census database that is used as one of the inputs (source zones) in the production of population grids (Figure 24(B2)).

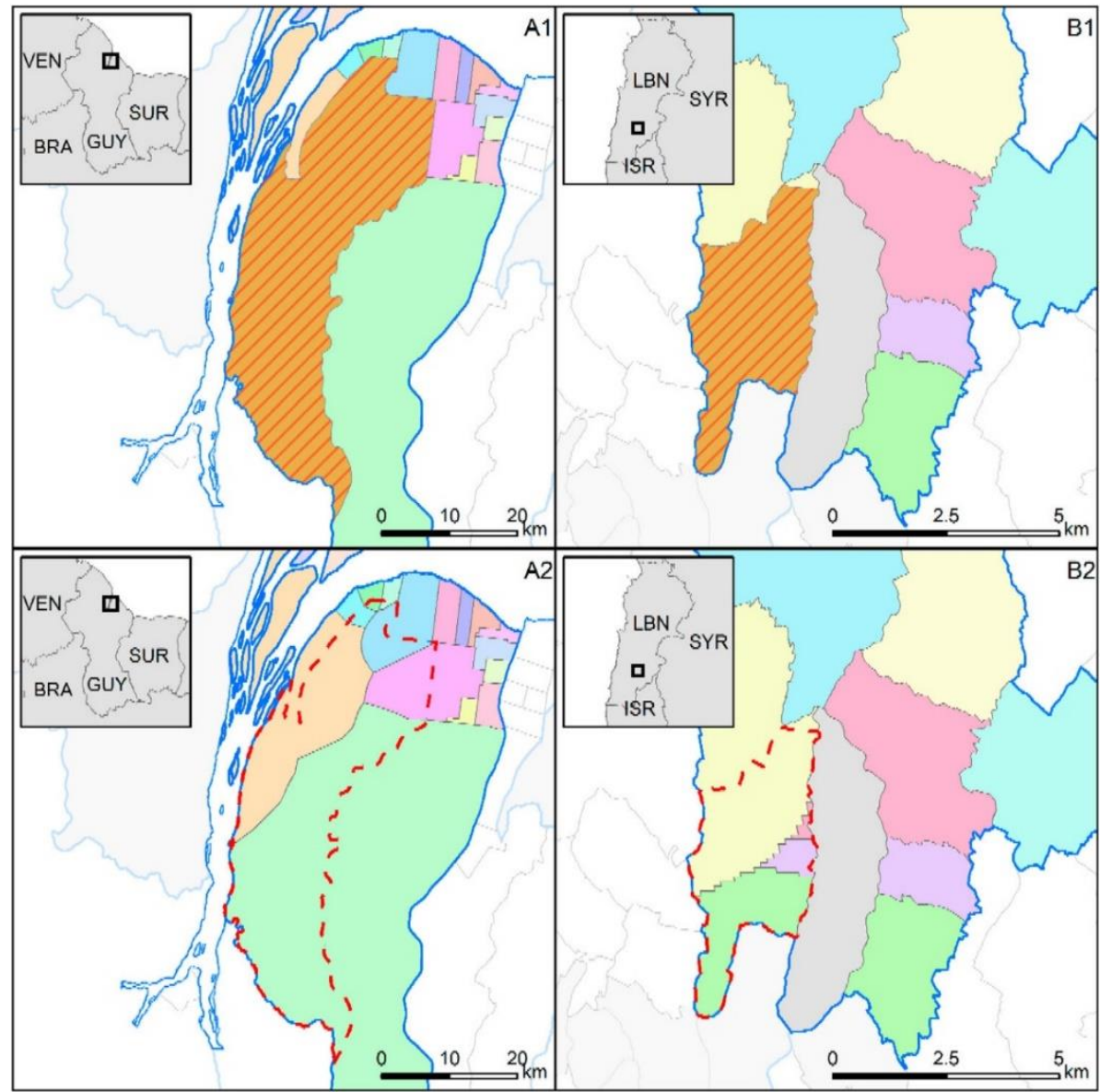

Figure 24 Examples of application of the 'split and merge' approach to census units incorrectly deemed as unpopulated in (A) Guyana and (B) Lebanon, before (1) and after (2) the procedure is applied. Orange filling and red dashed boundary represent the original problematic polygon; blue solid line delineates borders of upper administrative level; grey solid polygons are correctly declared unpopulated polygons; solid coloured areas are the resulting polygons; areas outside the processed administrative unit are shaded.

Ultimate results and benefits for population disaggregation are illustrated in Figure 25, for the same areas in Guyana and Lebanon, showing that final population distribution grids for year 2015 include populated cells in areas previously deemed as uninhabited. The procedure for mitigating and 're- 
populating' units previously unpopulated was implemented while limiting changes to the original geospatial census data. While in practice a new census geometry was created, the approach effectively minimizes changes to the source census database by:

(a) Minimizing modifications to the original geometry,

(b) Retaining the census hierarchy,

(c) Maintaining the regional distribution of population,

(d) Preserving the overall population counts.

The adopted approach is conservative: it decreases the population density in the zones corresponding to the original neighbouring populated units by expanding their area, while preserving their population. This approach is most suitable for the case when the population in the problematic unit was enumerated but re-assigned to other neighbouring units on reporting, thus retaining an accurate country population. However, if these populations were not enumerated to begin with, the country total will be inherently incorrect, which constitutes a more challenging case. Mitigation of the latter anomaly would require estimation of population actually residing in the unit using a bottom-up approach (see Wardrop et al. 2018 for options and discussion). Moreover, sources of population counts are notoriously old and outdated for some countries (e.g. Lebanon, DRC). It is possible that some units became populated or were settled after the census or estimates were conducted, as may be the case with refugee camps in Lebanon. It is also conceivable that some units, at the time the enumeration or estimates were produced, included settlements which were uninhabited (for a number of reasons, including war or other crises). Some areas are obviously densely populated (e.g. Sadr City in Baghdad, Iraq); their omission is most probably due to political and/or religious conflicts.

Problems of coverage and completeness in censuses are not new and their causes may be multiple and varied, including practical and political reasons (Carr-Hill 2013), and would certainly deserve more investigation that is beyond the scope of this paper. While there is some research reporting the general problem of undercounts (see Carr-Hill 2017 for summary), especially of particular groups, there is much less on the causes for complete and flagrant omission of population in large areas. In any case, the process of projecting population counts for a recent target year and subsequent adjustment to UN estimates, as done by GPW, does not 'populate' a census unit originally devoid of resident population because the adjustment is accomplished at the national level. 


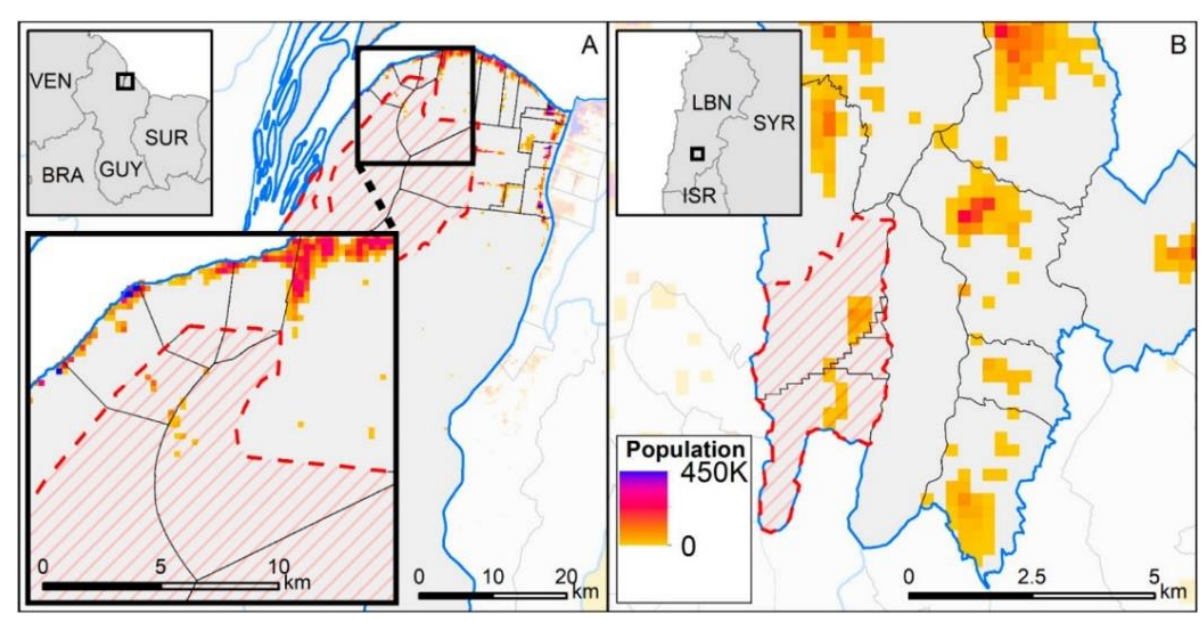

Figure 25 Illustration of resulting 250-m population grids for 2015 in (A) Guyana and (B) Lebanon

\subsubsection{Harmonization of population and settlement data along coastlines}

The semi-automated procedure to detect discrepancies between census data in GPWv4 and GHSL along coastlines (including inland water bodies) resulted in the detection of 287 patches having at least $1 \mathrm{~km} 2$ built-up area based on GHSL BU 2014 (totalling nearly $760 \mathrm{~km} 2$ ). The systematic visual inspection using VHR imagery revealed that 197 patches (containing $591 \mathrm{~km} 2$ or $77 \%$ of the inspected built-up area) had correctly identified areas with significant builtup structures, located in 25 countries across the globe. In the remaining 90 patches, built-up structures could not be identified or their presence was not significant. Figure 26 shows the location of the 197 patches where built-up presence was confirmed, showing issues clustered in Europe and in E Asia. As reported in Section 2.3, two procedures were followed for mitigating the confirmed discrepancies along coastlines, one manual and another automated. Initially the manual procedure was adopted, but the automated 'split and merge' procedure became more expeditious when the issue was found to be more systematic than initially assessed, as in case of Japan and the coast of the Ukraine. 


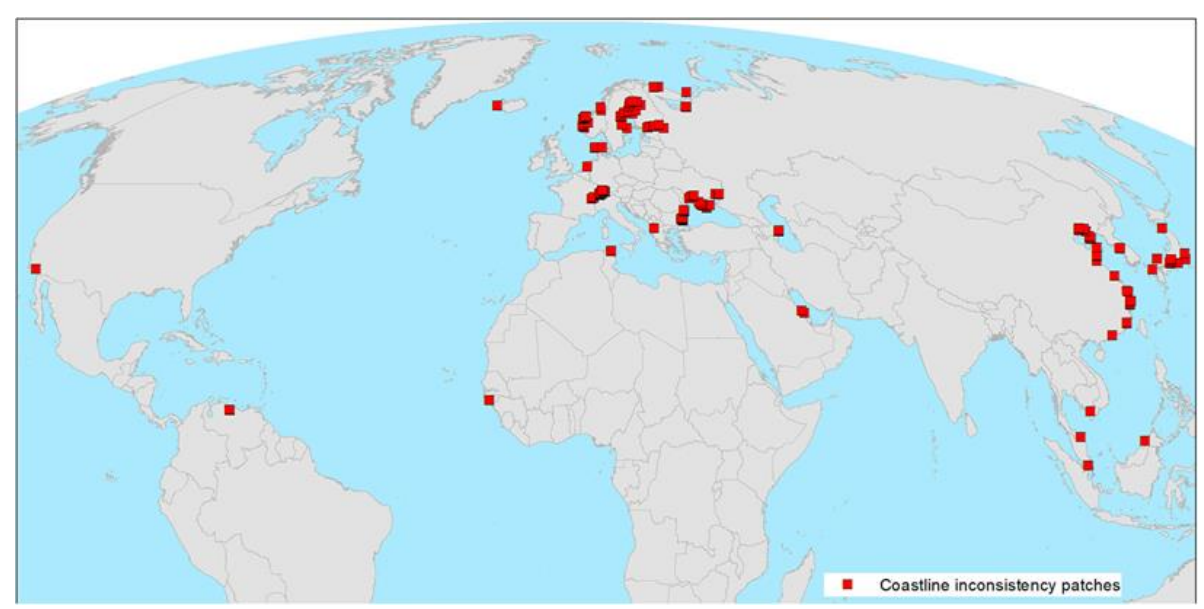

Figure 26 Visually confirmed coastline discrepancy patches larger than $1 \mathrm{~km}^{2}$.

Manual harmonization was carried out in the following countries: Albania, Austria, Azerbaijan, Bulgaria, Bahrain, Germany, Denmark, Dubai, Finland, Guinea-Bissau, Iceland, Republic of Korea, Malaysia, Netherlands, Norway, Romania, Russia, Singapore, Sweden, Tunisia, USA, Venezuela, and Viet Nam. Figure 27 illustrates the outcome of manual harmonization of coastline in Russia.

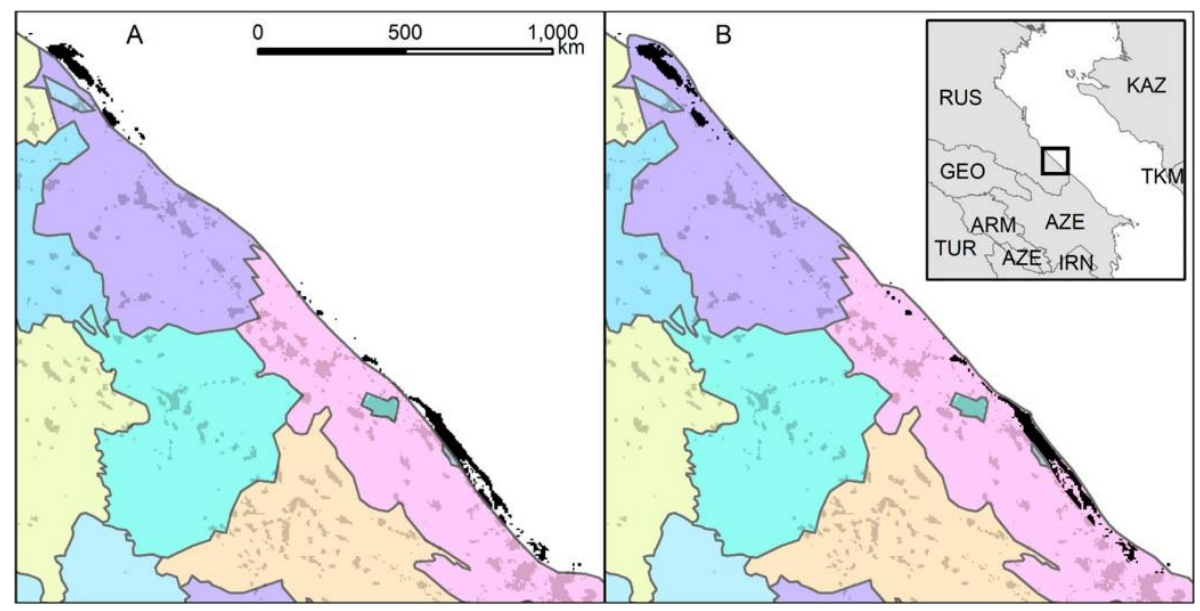

Figure 27 Example illustrating situation (A) before and (B) after manual harmonization of a stretch of Caspian Sea coastline in Russia. Solid black lines enclose original census units; black pixels denote mapped built-up areas (shaded: already within census boundaries; solid: outside original census areas).

The automated 'split and merge' approach was employed to modify and reconcile coastlines in Switzerland, France, Ukraine and Japan. Figure 28 shows 
the automated harmonization of coastlines applied along a stretch of Black Sea coast in Ukraine.

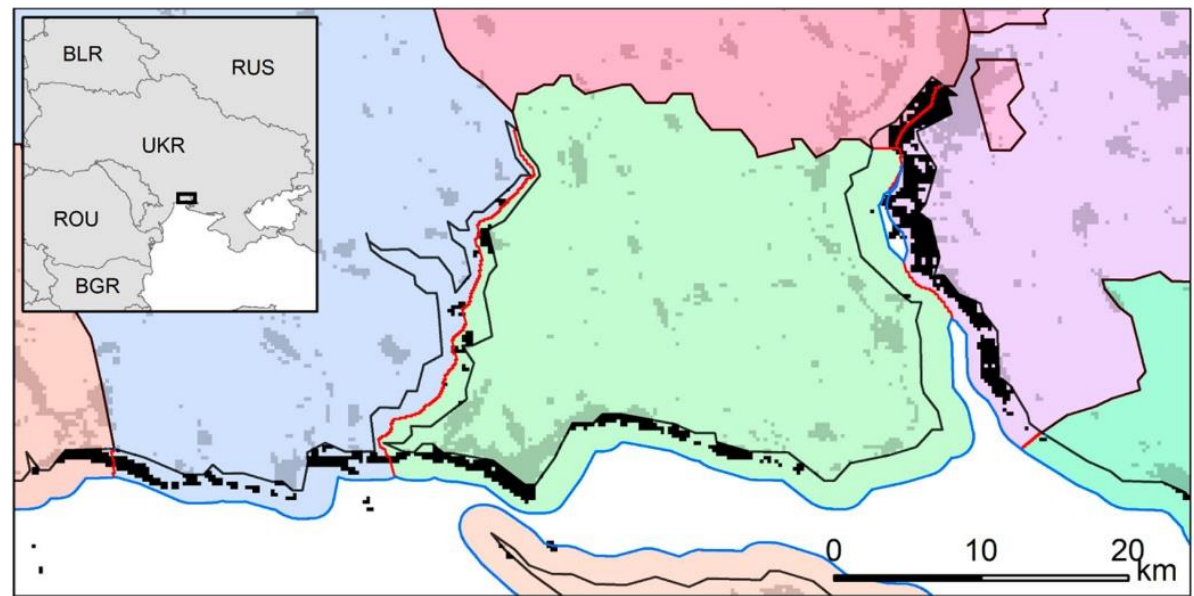

Figure 28 Example of automated approach to mitigate inconsistencies along the coastline of Ukraine. This example shows the generation of a 2-km buffer that was split using the 'split and merge' approach. Solid black lines enclose original census units; solid blue line represents the extended boundary into the sea through 2-km buffer; solid red lines represent the splitting of buffer into parts assigned to the nearest neighbouring unit; black pixels represent mapped built-up areas (shaded: already within census boundaries; solid: outside original census areas).

Although the detected anomalies may be significant for some applications such as the present one, and relevant from the perspective of data consistency, not all necessarily constitute errors in the original source data. Both in the case of coastline deficiencies and for units declared as unpopulated, the discrepancies may be due to anachronism between data sources. Coastal zones are typically attractive and dynamic areas from a settlement perspective, and geospatial data on administrative divisions used to support population estimates may not reflect the current land extents and settlement expansion obtained by land reclamation. Significant settlement expansion through land reclamation has been taking place in Asia (especially in China) and in the Middle East and it is important that time series of population grids capture and represent that process. Also, settlements can extend over water bodies such as floating settlements thus being located beyond the formal coastline. These can and are captured by GHSL, as no land mask is imposed a priori to limit detection of built-up. Instead of adopting the classical approach rooted in topography (e.g. Anderson et al. 1976) of following a hierarchical top-down procedure in abstracting the Earth surface into categories, GHSL adopts a people-centric approach that departs from the detection of human presence, and only then classifies phenomena into higher abstraction levels such as settlement classes (Pesaresi and Ehrlich 2009) (for explanation of GHSL family of products and 
analysis see Melchiorri et al. 2018). This approach may be a better match for the census paradigm, which aims to first identify where all people are so they can be surveyed and characterized. In this capacity, perhaps GHSL mapping of built-up could assist in planning census campaigns, especially in remote areas of countries having dynamic population trends that have not conducted a census in a long time.

Regarding the many discrepancies observed along coastlines, it is possible that these originate in differences in scale and level of detail among datasets, and at times can be introduced by the coarser detail of the database of Global Administrative Areas (GADM 2011) when boundaries are adjusted to a global framework in the GPW workflow.

Finally, it is important to note that the built-up structures detected and mapped could include abandoned or seasonal settlements, or otherwise non-residential facilities (e.g. ports, industrial areas). Ideally, a combination of census' spatial detail and attributes or ancillary data would allow further discrimination of land use according to application needs. In practice, the two addressed anomalies can be regarded as an expression of the same underlying issue, i.e. complete omission of population counts (due to undercounting/under-reporting or poor census geography) against contradicting evidence. It has been shown that a similar approach can be used to automatically mitigate systematic issues of both types.

\subsection{Conclusions}

Global population distribution grids are increasingly required and relied upon for many applications. These datasets are mostly produced by gridding or disaggregating counts from official population census and estimates. Despite the continuous advancements in population gridding methods, these usually do not address ex-ante issues affecting input population statistics. Availability, quality and detail of national data reporting on population is highly heterogeneous, and the best available global geospatial census data is not immune to shortcomings. These propagate to derived population distribution grids and may negatively impact their applications, in an age of new Development Agendas whose effective monitoring relies heavily on accurate and reliable geospatial population data and calls for universal inclusiveness of people. Exploring the improved capacities of recent global settlement data derived from remote sensing, we have sought to improve the mapping of population distribution by addressing some of these shortcomings.

New automated procedures to detect and mitigate major discrepancies and anomalies occurring in geospatial census data were developed, tested and implemented, while minimizing changes to the original data. Global and consistent remote sensing-derived data reporting on built-up presence was used to revise census units deemed as 'unpopulated' and to harmonize 
population distribution along coastlines. The two procedures employed for the detection of deficiencies in global geospatial census data obtained high rates of true positives, after validation and confirmation. The results also show that the targeted anomalies were significantly mitigated and that the baseline census database has improved, potentially benefitting other uses of the same statistical base. These outcomes are encouraging for further uses of free and open geoinformation derived from remote sensing. However, it must be recognized that assessing and monitoring progress of the new Development Agendas involves measuring many other population characteristics beyond population counts, for which proper census and surveys will remain invaluable sources, and that even accurate total population counts will ultimately continue to rely on high-quality enumerations and estimates.

This work illustrates the value and possible contribution of detailed, updated, and independent remote sensing data to complement and improve conventional sources of fundamental population statistics. While we acknowledge that the proposed procedures reduce the independency in the production of the involved variables (population and built-up distribution), which may be desirable for subsequent combination and production of population grids, these processes contribute in other ways by closing data gaps, improving data quality, and will ultimately benefit global mapping of population and its downstream applications. Future developments will focus on improving the automated detection and mitigation of deficiencies present in geospatial census data, including addressing additional anomalies (e.g. overreporting of population and extremely high population densities). The multitemporal nature of the data involved further increases the complexity of issues and adds challenges that should also be tackled, such as the estimation of population counts across epochs. For mitigation of areas incorrectly labelled as unpopulated, alternatives to the 'split \& merge' approach should be explored, namely contextual bottom-up approaches that take into consideration the local patterns of settlement.

Acknowledgements: The term country also refers, as appropriate, to territories or areas, and does not imply recognition of borders or legal status by the European Commission. 


\section{Chapter 6. An Improved Global Analysis of Population Distribution in Proximity to Active Volcanoes ${ }^{38}$}

38 This chapter is published as:

Freire, S.; Florczyk, A.J.; Pesaresi, M.; Sliuzas, R., 2019. An improved global analysis of population distribution in proximity to active volcanoes, 1975-2015. ISPRS Int. J. Geo-Inf., 8(8), 341. 


\begin{abstract}
:
Better and more detailed analyses of global human exposure to hazards and associated disaster risk require improved geoinformation on population distribution and densities. In particular, issues of temporal and spatial resolution are important for determining the capacity for assessing changes in these distributions. We combine the best-available global population grids with latest data on volcanoes, to assess and characterize the worldwide distribution of population from 1975-2015 in relation to recent volcanism. Both Holocene volcanoes and those where there is evidence of significant eruptions are considered. A comparative analysis is conducted for the volcanic hot spots of Southeast Asia and Central America. Results indicate that more than $8 \%$ of the world's 2015 population lived within $100 \mathrm{~km}$ of a volcano with at least one significant eruption, and more than 1 billion people (14.3\%) lived within 100 $\mathrm{km}$ of a Holocene volcano, with human concentrations in this zone increasing since 1975 above the global population growth rate. While overall spatial patterns of population density have been relatively stable in time, their variation with distance is not monotonic, with a higher concentration of people between 10 and $20 \mathrm{~km}$ from volcanoes. We find that in last 40 years in Southeast Asia the highest population growth rates have occurred in close proximity to volcanoes (within $10 \mathrm{~km}$ ), whereas in Central America these are observed farther away (beyond $50 \mathrm{~km}$ ), especially after 1990 and for Holocene volcanoes.
\end{abstract}

\title{
6.1 Introduction
}

Volcanic eruptions are among the most powerful and destructive natural hazards on Earth, capable of causing widespread human suffering and extensive economic losses, both from direct impacts and through the disturbances to livelihoods arising from indirect or cascading effects (e.g., via disruptions of air transport, through originating tsunamis, etc. (UNISDR 2015b). Severe eruptions can cause rapid global changes by modifying weather patterns, disrupting climate, and, as in the past, induce mass extinctions of species (National Academy of Sciences, 2017). Although moderate to large volcanic eruptions are infrequent, these are potentially high-consequence incidents, especially for nearby populations due to lava flows, ash deposition, pyroclastic events, and other phenomena (Dilley et al. 2005). Still, the 2010 eruption of Eyjafjallajökull volcano in Iceland demonstrated that even modest eruptions in relatively remote locations can have global impacts (National Academy of Sciences, 2017, Ragona et al. 2011). Volcanic activity can have a disproportionate effect on livelihoods and economy because of high systemic vulnerability (Poljanšek et al. 2017).

Volcanoes located in densely populated areas, however, do not need to have powerful eruptions to produce devastating effects. In such settings, even small-to moderate-sized eruptions may constitute severe threats, by 
generating multiple hazards which can affect areas from the immediate vicinity up to hundreds of kilometres distance (for detailed discussion see Chester et al. 2000, Brown et al. 2017). In a study of past disasters, residents make up the largest number of fatalities (Brown et al., 2017).

Beyond their destructive power, volcanoes and volcanic activity can also benefit humans and their livelihoods. Among their major beneficial effects are the provision of nutrients to the surrounding soil, favoring agricultural productivity-an important aspect in agrarian societies-but also economic activities such as resource extraction (e.g., Sulfur mining), tourism, and geothermal energy (National Academy of Sciences, 2017). Such benefits help to explain population concentrations in their vicinity. In addition, tropical volcanoes provide climatic advantages relative to surrounding regions (Small et al., 2001), which may have promoted human settlement and the clustering of people. Such clustering in proximity to active volcanoes creates exposure levels with changes that vary in space or time (Strader et al., 2015). This exposure combines with existing vulnerabilities to generate risk and the potential for losses. Evidence indicates that exposure of persons and assets in all countries has increased faster than vulnerability has decreased, thus generating new risks (UNISDR, 2015).

Evaluating potential or actual human exposure for global disaster risk assessment is as essential as it is challenging, being limited by the availability and quality of geophysical and socio-economic data (Lerner-Lam, 2007, Peduzzi et al. 2009a). As a result, previous estimations of human exposure were typically conducted for small areas and without a temporal dimension, or merely approximated with coarse population geoinformation ( $\sim 1 \mathrm{~km}$ grids). However, the rapid increase in exposure of population and assets is currently a major driver of growing disaster risk (UNISDR, 2013), requiring adequate global geoinformation layers depicting the distribution and densities of population. Such geoinformation is valuable throughout the emergency management cycle, but for timely and efficient response it should be prepared and made available beforehand (Freire, 2010). After a disaster event, the quality and granularity of population data have a direct effect on response and lives saved (National Research Council, 2007). Although global population grids have been produced since the 1990s, they have suffered from some issues which limit their usability (Linard et al. 2012, Mondal et al. 2012). Among these are their coarse spatial resolution, incomplete discrimination of settlements (especially smaller ones), and the use of dichotomous maps of built-up in their construction. Also, for change detection and analysis, a coherent and robust spatio-temporal production model is required to allow objective comparisons.

Concerning volcanic risk, Andredakis et al. 2015 highlight that risk elements are on the rise, most notably population exposure. The shifting focus of urbanization from developed to developing countries taking place in recent decades is also increasing global exposure to natural hazards, including 
volcanism (Chester et al. 2000). Volcanic activity remains a constant threat globally and the number of people at risk from volcanism is rising with increased urbanization and population growth (National Academies of Sciences 2017, Chester et al. 2000, Brown, et al. 2017, Ewert 2004). However, volcanic risk analysis and assessment at the global scale is not as advanced as for other hazards such as flooding, earthquakes and tropical cyclones (Poljanšek et al. 2017), being limited by the availability and quality of data (Pan et al. 2015), especially regarding their consistency and detail. Similarly to earthquakes, volcanic eruptions are difficult to mitigate technologically (Riede, 2016), in that the hazard component can hardly be decreased by human action. While expected losses may be lower than those from other hazards at a global scale, in affected regions, they can be very significant. Since 1950, an average of 31 volcanoes have erupted each year (United Nations, 2015), and at any one time at least 20 are erupting (Siebert et al. 2010, Siebert et al. 2015). Given this context, major gains to disaster risk reduction are to be obtained through reductions in vulnerabilities and especially in exposure, requiring improved assessments of these components.

\subsubsection{Potential Global Population Exposure to Volcanic Hazard - Narrowing Data Gaps}

Several assessments of potential worldwide population exposure to volcanic hazard have been conducted using globally-available population grids, e.g., (Dilley et al. 2005, Small and Naumann 2001, Aspinall et al. 2011, Brown et al. 2015). However, most such studies are now relatively outdated, or use coarse population geoinformation. Often, assessments are based on data that are neither open nor free. Small and Naumann (Small and Naumann 2001) in their landmark work quantified human proximity to both Holocene and historically active volcanoes for 1990 , but underlined that the spatial resolution of population data used (GPWv2) imposes limitations on the analysis and is not adequate for detailed assessment of potential volcanic risk. Ewert and Harpel (Ewert et al. 2004) have proposed a Volcano Population Index (VPI) to make objective estimates and comparisons of the number of people at risk from volcanic hazards. Within a given radius of a volcanic vent, the index quantifies one component of risk-population-from one volcano to another. The authors have noted that the 1-kilometer grid size of the population grid used (LandScan) was the principal limiting factor in making precise estimates of population at risk (Ewert et al. 2004). The Population Exposure Index (PEI) (Aspinall et al. 2011) is one of the prominent indices used in assessing volcano risk. It is based on the population within 10,30 , and $100 \mathrm{~km}$ of a volcano, which is then weighted according to evidence on historical distributions of fatalities within a given distance from volcanoes. The PEI is divided into seven levels, from sparsely to very densely populated areas. The authors have stated that the main drawback of such methods is that they rely on a number of parameters having high uncertainty or that are simply unknown for many 
volcanoes. This limits application of the method mainly to those with good historical records (Scandone et al. 2016). As a simpler and more direct way to compare different volcanoes or volcanic areas, (Scandone et al. 2016) have proposed a volcanic risk coefficient (VRC) using the number of people that may be affected by the maximum expected eruption as a main variable. In addition to the estimation of this specific hazard information, this still requires detailed and comparable population distribution data.

Common to all these efforts is the need for up-to-date and spatially-explicit population distribution data, which are globally-consistent and have sufficient resolution to support detailed analyses and comparisons. This is especially important as databases of locations and features of potential volcano hazard (i.e., GPV) are also subject to frequent revisions and updates, thereby inviting parallel re-assessments of global and regional potential population exposure.

Improved modeling and mapping of global population distribution can be achieved with remote sensing imagery and methods, which have been evolving towards a more detailed, objective and independent data source on the human presence on the Earth surface (Freire et al. 2018a). The multiscale synoptic perspective is considered one of the core strengths of remote sensing when it comes to questions of urban and socio-economic development and in regards to the closely interlinked disaster risk domain (Aubrecht et al. 2017b). In the specific case of population exposure to volcanic hazard, (Brown et al. 2017) point out that "moderate resolution satellite imagery, such as that provided by the Landsat series, could provide more spatially explicit quantifications of settlement patterns in the vicinity of potentially active volcanoes. More recently, (Pittore et al. 2017) advocate for full exploitation of rapid, large-scale data collection based on remote sensing, while also stressing that dissemination of exposure data is key to empowering end-users and communities and for allowing and encouraging risk evaluation.

\subsubsection{The Contribution of the Global Human Settlement Layer (GHSL) to DRM}

The recent application of new cost-effective, automated and fully replicable data classification methods (e.g., symbolic machine learning) to contemporary open and free global satellite imagery collections have enabled the emergence of a new generation of geospatial data derived from remote sensing, led by the Global Human Settlement Layer (GHSL) (Pesaresi and Ehrlich 2009). These developments allowed the global mapping of built-up areas and settlements with unprecedented spatio-temporal detail and accuracy (Pesaresi et al. 2009, Pesaresi et al. 2013, Pesaresi et al. 2016), under a set of pragmatic requirements and principles summarized in (Melchiorri et al. 2019). Among other uses, these new datasets are providing an effective contribution to population disaggregation and other population distribution modeling efforts, especially due to a combination of: 
- Semantic robustness and interoperability

- Currency of data

- Spatial detail

- Global coverage

- Temporal depth

- Provision of open and free data

GHSL's enhanced spatio-temporal mapping of buildings and density of built-up areas offers a suitable proxy for the location of people and, as such, is being used to improve the disaggregation of census data into derived population grids (Freire et al. 2015d, Freire et al. 2016a, Linard et al. 2017, Nieves et al. 2017, Balk et al. 2019).

From its inception, development of GHSL was driven by requirements for postdisaster and post-conflict damage, needs and reconstruction assessment, including refugee camps and temporary, rapidly-changing human settlement monitoring (Pesaresi et al. 2015c). Therefore Disaster Risk Management and Reduction is a major field of application of GHSL data, and among the uses of its population distribution grids (GHS-POP) are the baseline estimation of multi-temporal exposure to global hazards (Pesaresi et al. 2017, Ehrlich et al. 2018b) and assessment of impacts from climate hazards (Ehrlich et al. 2018a), including the current and future projections of risk of river flood (Alfieri et al. 2017) (https://www.efas.eu; http://www.globalfloods.eu), and support to creation of risk indices for management for humanitarian crises and disasters (e.g., INFORM-Index for Risk Management (http://www.inform-index.org).

The GHSL layers and especially population distribution data enable conducting more updated, refined, and longitudinal assessments of population potentially affected by different types of natural hazards (Ehrlich et al. 2018b), including threats posed by volcanoes (Freire et al. 2016b). Due to their characteristics, it is expected that these new population data can further advance, update, and detail assessment of population distribution in the vicinity of active volcanoes and contribute to enhance estimations of potential exposure to this type of hazard.

In this article, we aim to characterize with unprecedented detail the changes in the distribution of human population in proximity to historically active volcanoes, focusing on the last 40 years. We do that by combining detailed geospatial grids of global population densities for the 1975-1990-2000-2015 period with the latest data on distribution of volcanoes on Earth, considering both Holocene volcanoes and those having significant eruptions. We investigate the recent evolution and current distribution of population in relation to historical volcanism and discuss how overall potential population exposure and density patterns have been varying with distance. More detailed attention is dedicated to Southeast Asia and Central America, where concentrations of people in vicinity of volcanoes are greater than in other 
volcanic regions. This work details and updates preliminary research done by Freire et al. 2016b and shows the value of using the more recent datasets in future assessments of volcanic risk.

\subsection{Materials and Methods}

In Section 6.2.1., we present and discuss the datasets used, including a description of the production and features of the global population grids that made this study possible, and the global datasets of volcanoes. In Section 6.2.2 we present the methodology that was applied for assessing global population distribution in relation to historical and significant volcanism.

\subsubsection{Data}

\subsubsection{Global Population Distribution Grids 1975-2015}

In order to assess population distribution in relation to distance to volcanoes, we have used population grids for 1975, 1990, 2000, and 2015 produced in the frame of the Global Human Settlement Layer (GHSL) project. These grids (GHS-POP), reporting the distribution and density of total resident population per 250-m cell, are created by using maps of density of built-up areas (Pesaresi et al. 2016) for matching epochs as proxy for disaggregating best-available population estimates (Freire et al. 2016a).

GHS-POP is based on population estimates provided by CIESIN's Gridded Population of the World (GPW) v4.10 for matching periods (CIESIN, 2016), with national figures adjusted to UN World Population Prospects (DoxseyWhitfield, 2015). CIESIN collects and integrates the most recent and finest resolution census data available, and the CIESIN-GPWv4.10 input dataset includes the latest round of censuses (2010-2011) and comprises more than 12 million census or administrative units, with average spatial resolution varying widely by country.

The GHS-POP population grids effectively harmonize and increase the resolution of this variable in space and time by using consistent ancillary data and methods. Among the specific methodological features of these grids are:

(i) the use of a single spatially and temporally-explicit proxy (built-up areas from GHSL);

(ii) this proxy is mostly of higher spatial resolution than census data and is derived with a consistent approach;

(iii) the employment of a simple, transparent and consistent methodology for population disaggregation.

GHS-POP is also unique among current global population grids in that cell values represent both counts and densities of population, by using as native projection the World Mollweide equal-area projection. The latest release of GHS-POP used in this study (made publicly available in 2019), mitigates 
important shortcomings of previous population grids by improving representation of distributions along coastlines and critically revising areas declared as 'unpopulated' but where significant evidence of human habitation existed (Freire et al. 2018a). Therefore GHS-POP is especially suited for supporting Disaster Risk Management (DRM) tasks by refining distribution of resident population to known places of human shelter and activities (i.e., buildings), where most people spend the majority of their time. Due to these characteristics and given that GHS-POP is currently the only global dataset that spans the last 40 years with comparable, detailed, open, and free data on distribution of population in settlements, it allows investigating population distribution in proximity to volcanoes in this period.

\subsubsection{Global Geographical Distribution of Volcanoes}

The proximal analysis of population was conducted considering two widely used global datasets of volcanoes, available online: the Holocene Volcano List v. 4.7.6 (Volcanoes of the World 4.7.6.) of the Smithsonian Institution's Global Volcanism Program (GVP) (Global Volcanism Program, 2013) and the NOAA Significant Volcanic Eruption Database (NOAA, 2019), this one also based on GVP.

The Holocene Volcano List (HV in this article) is a global listing of volcanoes believed to have been active during the Holocene period (the last $\sim 10,000$ years). This database includes information on the latitude, longitude, elevation, type of volcano, and last known eruption. The HV database is frequently updated and v. 4.7.6 used in this work includes 1432 volcanoes, of which 118 are submarine volcanoes and 83 correspond to volcanic fields.

The Significant Volcanic Eruption Database (SV in this article) is a global listing of over 500 significant eruptions. The current version used here lists 826 eruptions for 265 volcanoes. This database includes information on volcanoes (i.e., latitude, longitude, geographic location, elevation, type of volcano), their eruptions (date of eruption, the assigned Volcanic Explosivity Index (VEI) and associated events, such as tsunami and/or earthquake), and impact (fatalities, estimated economical damage, etc.). A significant eruption is defined as one that meets at least one of the following criteria: caused fatalities, produced moderate damage (approximately 1 million USD or more), had a Volcanic Explosivity Index (VEI) of 6 or larger, caused a tsunami, or was associated with a major earthquake (NOAA, 2019). Figure 29 shows the global distribution of volcanoes from the above databases that were considered in this study. 


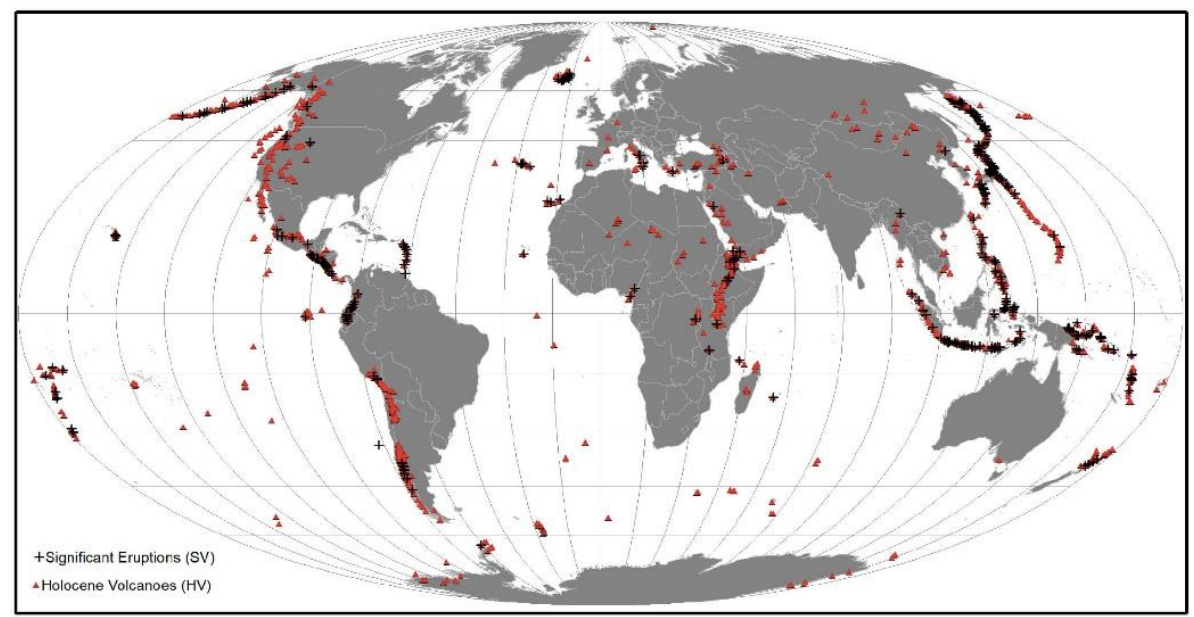

Figure 29 Distribution of the two global datasets of volcanoes used in this study: Holocene Volcano List v4.7.6 (HV) and Significant Volcanic Eruption Database (SV) (World Mollweide projection).

\subsubsection{Assessing Global Population Distribution and Volcanism}

We analyzed global population distribution as a function of distance to volcanoes in 1975, 1990, 2000, and 2015, using the $250 \mathrm{~m}$ population grids (GHS-POP) for the respective epochs. Using GIS software, population distribution was analyzed up to a radial distance of $100 \mathrm{~km}$ from volcanoes, by buffering each volcano in $1 \mathrm{~km}$ steps and conducting zonal analysis of population grids. Given the global scope of this analysis and the challenges involved with measuring accurately both distances and areas (impossible in same cartographic projection), and the fact that population grids are produced in equal-area projection (thus representing both counts and densities), we devised the following procedure:

(1) Calculation of distance buffers from volcanoes in World Equidistant Cylindrical projection;

(2) Merging the resulting buffers by distance (i.e., dissolve by distance function);

(3) Re-projection of the distance buffers to World Mollweide projection;

(4) Rasterization to the working grid (i.e., $250 \mathrm{~m}$ World Mollweide aligned with the GHS global grid), and calculation of landmass surface and population counts per each buffer in World Mollweide.

This approach tries to minimize distortions in distances, areas, and population counts. The considerable effort expended to maintain the accuracy of underlying databases is an aspect that is absent (or omitted) from previous global analyses (Dilley et al. 2005, Small and Naumann 2001, Aspinall et al. 
2011, Brown et al. 2015), therefore constituting a relevant new feature of the current analysis.

For discussing the results, four distances from volcanoes were selected as being especially relevant: $10,30,50$, and $100 \mathrm{~km}$. Aspinall et al. (2011) adopted 10 and $30 \mathrm{~km}$ in their PEI proposal. Moreover, $10 \mathrm{~km}$ is a frequently used distance for evacuation after moderate to large eruptions. Regarding the $50 \mathrm{~km}$ mark, (Brown et al. 2017) have identified that close to $100 \%$ of volcanic fatalities registered have occurred within this distance from volcanoes. $100 \mathrm{~km}$ is considered relevant as a maximum distance from volcanoes for assessing the direct effects of volcanic eruptions, since lethal pyroclastic flows and surges (Nakada, 2000), and lahars (Rodolfo, 2000) may occasionally extend to these distances. Among twelve destructive phenomena associated with volcanic eruptions, it is estimated that seven can potentially reach such a distance (Chester et al. 2000). Given the observation by (Small and Naumann 2001) that higher population densities in Southeast Asia and Central America lie in closer proximity to volcanoes than in other volcanic regions, we conduct a more detailed analysis of results for these regions. As in that work, volcanic regions are considered to be those with a significant number of persistently active volcanoes.

\subsection{Results and Discussion}

\subsubsection{Global Population Distribution from 1975 to 2015 in Relation to Volcanism}

This section presents and discusses the global distribution of population from 1975 to 2015 in relation to volcanism, considering both overall population in each period and annual growth rates by distance to both HV and SV. While the first part focuses on significant distance ranges up to $100 \mathrm{~km}$ of volcanoes, a second part analyses the specific spatio-temporal patterns of population density within $50 \mathrm{~km}$ of volcanoes in more detail.

Table 16 shows total accumulated population within selected distance ranges (radial distances) from Holocene volcanoes (HV), for each period considered. Results show that the total population living in proximity of Holocene volcanoes (HV) is very significant and that this amount has been increasing steadily in last 40 years. In 2015, more than 1 billion people, $14.3 \%$ of the global population, are estimated to live within a range of potential direct impact of volcanic eruptions (i.e., within $100 \mathrm{~km}$ ). Close to 60 million people are estimated to live within potential evacuation range worldwide (i.e., within 10 $\mathrm{km})$. 
Table 16 Population (in millions and as percentage of World total) by radial distance from Holocene volcanoes (HV) in 1975, 1990, 2000, and 2015.

\begin{tabular}{ccccccccc}
\hline & \multicolumn{2}{c}{$\mathbf{1 9 7 5}$} & \multicolumn{2}{c}{$\mathbf{1 9 9 0}$} & \multicolumn{2}{c}{$\mathbf{2 0 0 0}$} & \multicolumn{2}{c}{$\mathbf{2 0 1 5}$} \\
\hline $\begin{array}{c}\text { Dist. } \\
(\mathrm{km})\end{array}$ & $\begin{array}{c}\text { Pop. } \\
{\left[10^{6}\right]}\end{array}$ & $\%$ & $\begin{array}{c}\text { Pop. } \\
{\left[10^{6}\right]}\end{array}$ & $\%$ & $\begin{array}{c}\text { Pop. } \\
{\left[10^{6}\right]}\end{array}$ & $\%$ & $\begin{array}{c}\text { Pop. } \\
{\left[10^{6}\right]}\end{array}$ & $\%$ \\
\hline 10 & 29 & 0.7 & 41 & 0.8 & 49 & 0.8 & 59 & 0.8 \\
30 & 176 & 4.3 & 243 & 4.6 & 284 & 4.6 & 340 & 4.6 \\
50 & 301 & 7.4 & 415 & 7.8 & 489 & 8 & 592 & 8.1 \\
100 & 566 & 13.9 & 751 & 14.1 & 870 & 14.2 & 1054 & 14.3 \\
Global & 4,061 & 100 & 5,310 & 100 & 6,127 & 100 & 7,349 & 100 \\
\hline
\end{tabular}

Concerning population growth rates in vicinity of Holocene volcanoes (HV) (Table 17), these are generally decreasing with time, accompanying global population trends. These rates were especially high from 1975 to 2000, and were increasing with proximity to HV. However, despite slowing down with time, rates in vicinity of HV were largely above the global average in all periods.

Table 17 Compound annual growth rate of population by radial distance from Holocene volcanoes (HV) for each time interval.

\begin{tabular}{ccccc}
\hline $\begin{array}{c}\text { Distance } \\
(\mathbf{k m})\end{array}$ & $\mathbf{1 9 7 5 -}$ & $\mathbf{1 9 9 0 -}$ & $\mathbf{2 0 0 0 -}$ & $\mathbf{1 9 7 5 -}$ \\
\hline 10 & $\mathbf{1 9 9 0}$ & $\mathbf{2 0 0 0}$ & $\mathbf{2 0 1 5}$ & $\mathbf{2 0 1 5}$ \\
30 & 2.29 & 1.76 & 1.27 & 1.78 \\
50 & 2.19 & 1.58 & 1.19 & 1.66 \\
100 & 2.17 & 1.64 & 1.29 & 1.71 \\
Global & 1.90 & 1.48 & 1.29 & 1.57 \\
\hline
\end{tabular}

Table 18 and Table 19 refer to variation of population within selected distances of volcanoes with significant eruptions (SV). Results show that this population has also been increasing steadily and amounts to 616 million in 2015, or $8.4 \%$ of global population. Also very significant are the 21 million people living within $10 \mathrm{~km}$ of these volcanoes.

Table 18 Population (in millions and percentage of World total) by radial distance from volcanoes with significant eruptions (SV) in 1975, 1990, 2000, and 2015.

\begin{tabular}{ccccccccc}
\hline \multicolumn{2}{c}{1975} & \multicolumn{2}{c}{1990} & \multicolumn{2}{c}{$\mathbf{2 0 0 0}$} & \multicolumn{2}{c}{$\mathbf{2 0 1 5}$} \\
\hline $\begin{array}{c}\text { Distance } \\
(\mathrm{km})\end{array}$ & $\begin{array}{c}\text { Pop. } \\
{\left[10^{6}\right]}\end{array}$ & $\%$ & $\begin{array}{c}\text { Pop. } \\
{\left[10^{6}\right]}\end{array}$ & $\%$ & $\begin{array}{c}\text { Pop. } \\
{\left[10^{6}\right]}\end{array}$ & $\%$ & $\begin{array}{c}\text { Pop. } \\
{\left[10^{6}\right]}\end{array}$ & $\%$ \\
\hline 10 & 12 & 0.3 & 16 & 0.3 & 18 & 0.3 & 21 & 0.3 \\
30 & 87 & 2.2 & 115 & 2.2 & 130 & 2.1 & 150 & 2 \\
50 & 169 & 4.1 & 222 & 4.2 & 254 & 4.1 & 297 & 4 \\
100 & 333 & 8.2 & 444 & 8.4 & 515 & 8.4 & 616 & 8.4 \\
Global & 4,061 & 100 & 5,310 & 100 & 6,127 & 100 & 7,349 & 100 \\
\hline
\end{tabular}


Table 19 Compound annual growth rate of population by radial distance of volcanoes with significant eruptions (SV) for each time interval.

\begin{tabular}{ccccc}
\hline $\begin{array}{c}\text { Distance } \\
(\mathbf{k m})\end{array}$ & $\mathbf{1 9 7 5 -}$ & $\mathbf{1 9 9 0 -}$ & $\mathbf{2 0 0 0 -}$ & $\mathbf{1 9 7 5 -}$ \\
\hline 10 & $\mathbf{1 9 9 0}$ & $\mathbf{2 0 0 0}$ & $\mathbf{2 0 1 5}$ & $\mathbf{2 0 1 5}$ \\
30 & 1.90 & 1.43 & 1.03 & 1.45 \\
50 & 1.82 & 1.28 & 0.96 & 1.36 \\
100 & 1.86 & 1.36 & 1.05 & 1.43 \\
Global & 1.94 & 1.48 & 1.20 & 1.55 \\
\hline
\end{tabular}

Regarding population change with distance bands, growth rates are lower than in proximity to HV. Also differently from HV, population growth has been generally decreasing with time but also with proximity to SV volcanoes, except for the closest distance band $(10 \mathrm{~km})$. Only in the period 1975-1990 did population in these areas increase above the overall growth rate, having dipped below the global rate in the most recent period. In the period 2000-2015, population growth in these areas dipped below global growth, especially closer than $30 \mathrm{~km}$ from volcanoes. This may be related to: (i) the specific demographic trends of the countries and areas where SV volcanoes are located and/or (ii) with an increased awareness of the threat posed by these volcanoes and limitations to settlement in their vicinity.

Figure 30 and Figure 31 show overall global cumulative population as a function of radial distance to Holocene volcanoes (HV) and volcanoes in Significant Volcanic Eruption Database (SV), respectively, for each period under analysis. The plots show that very large numbers of people live in proximity to volcanoes, and that this presence has increased from 1975 to 2015. Concerning HV, while 100 million people would be spread over a radial distance of $20 \mathrm{~km}$ in 1975, the same amount were concentrated in a radius of only 13 $\mathrm{km}$ in 2015 . This has noteworthy consequences for the variation of population densities in space and time, as shown in Figure 32 and Figure 33. 


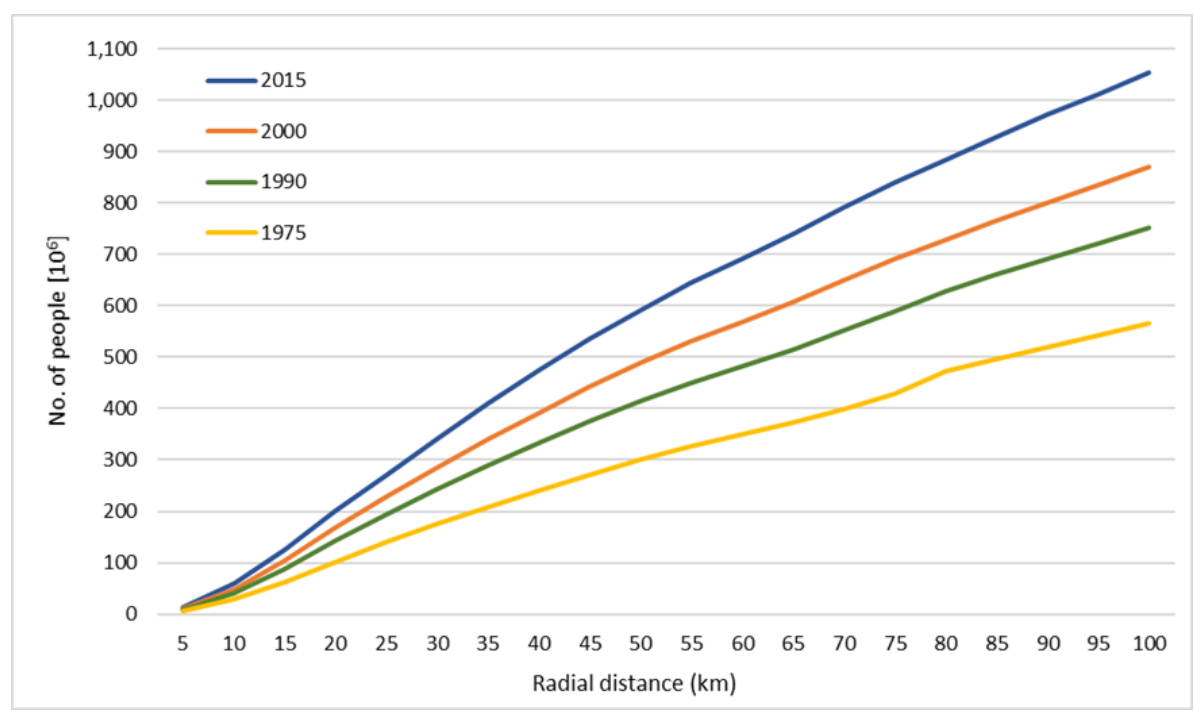

Figure 30 Cumulative population as a function of radial distance to Holocene volcanoes (HV), in 1975, 1990, 2000, and 2015.

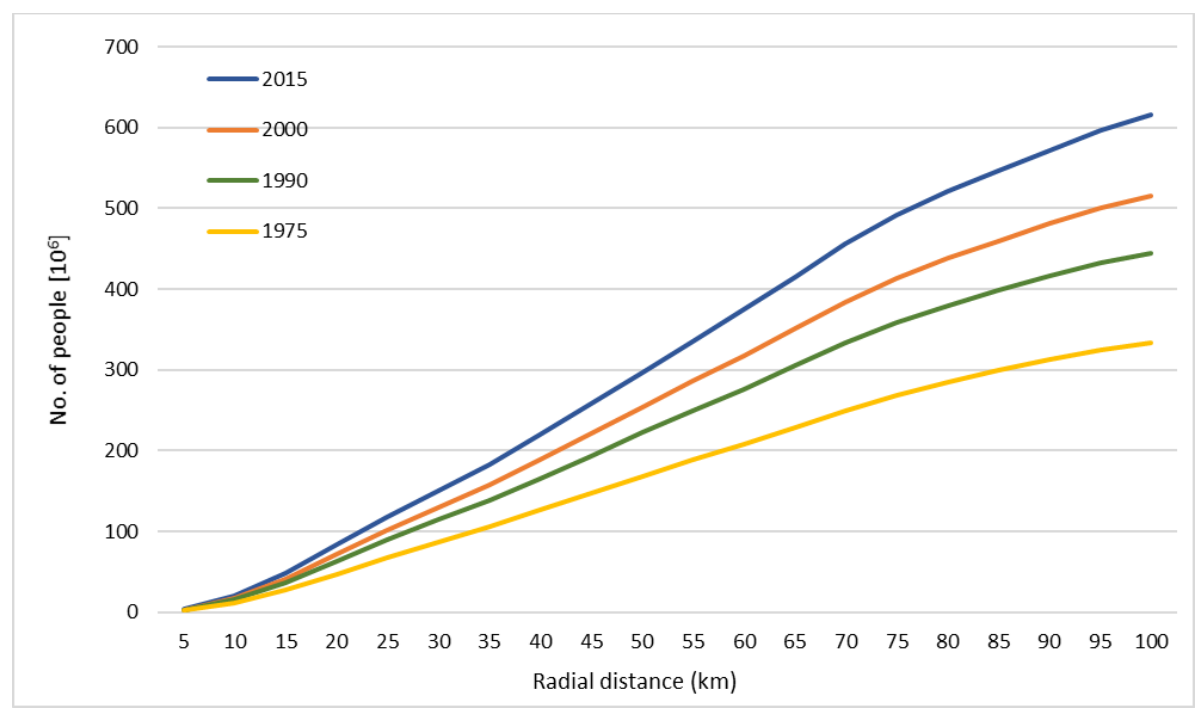

Figure 31 Cumulative population as a function of radial distance to volcanoes in Significant Volcanic Eruption Database (SV), in 1975, 1990, 2000, and 2015. 


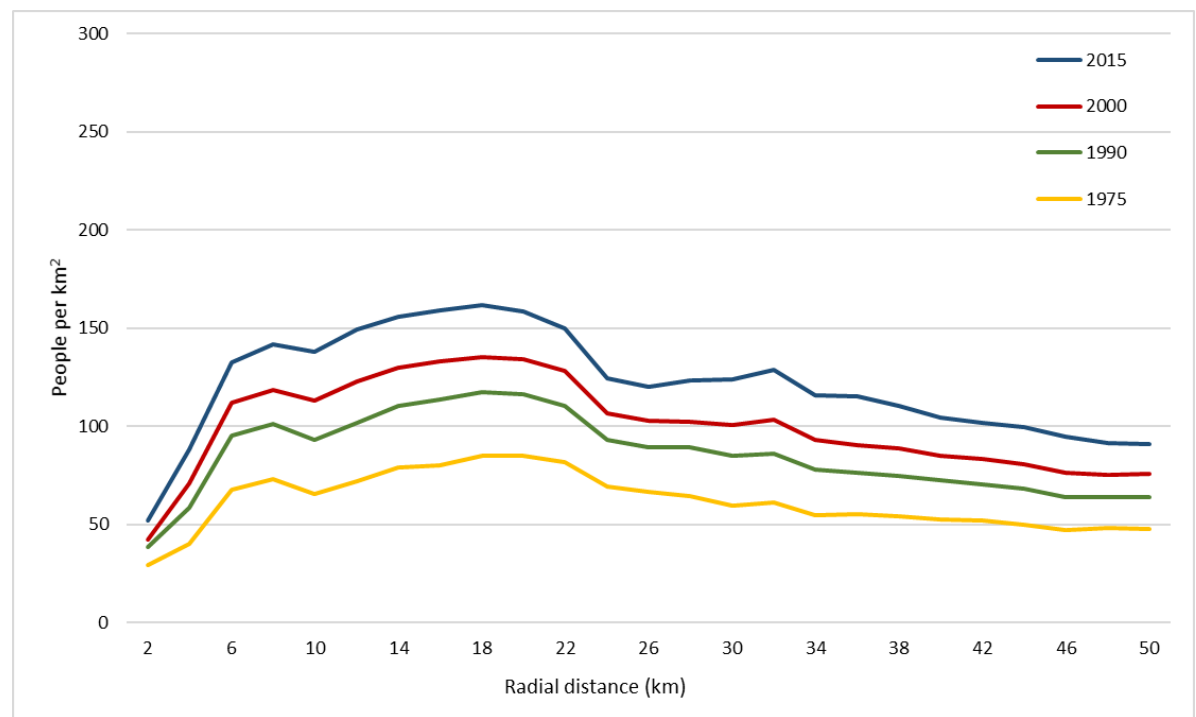

Figure 32 Average population density as a function of radial distance to Holocene volcanoes (HV), in 1975, 1990, 2000, and 2015.

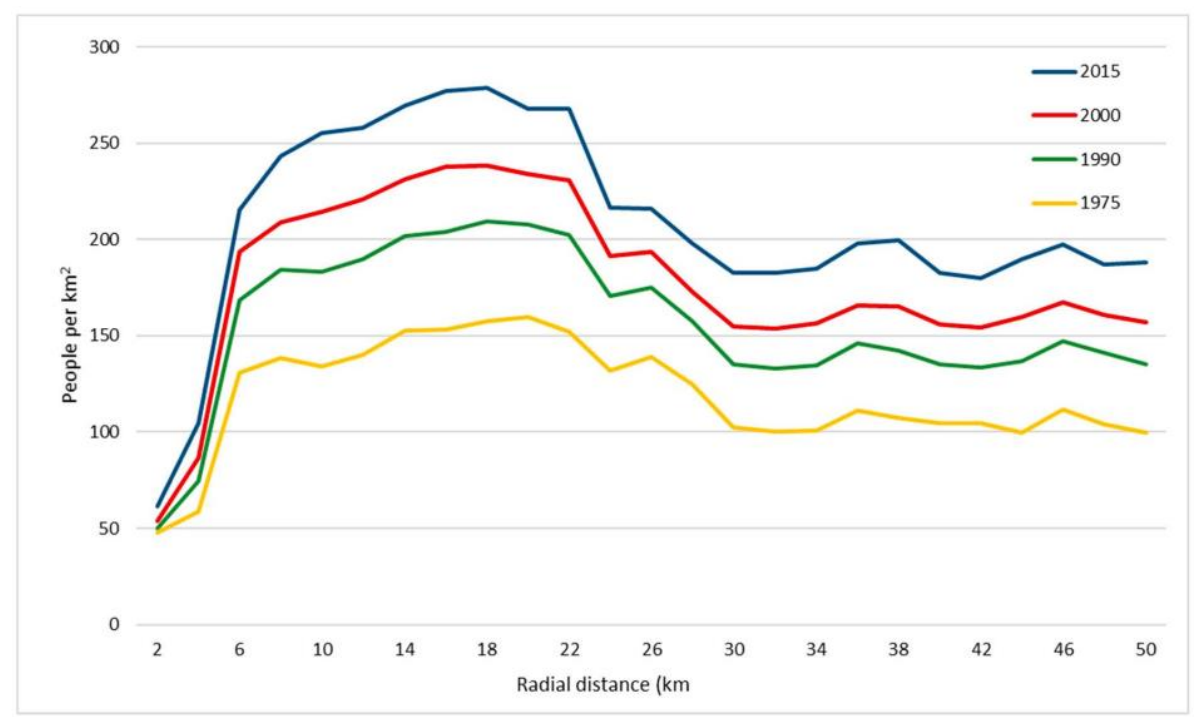

Figure 33 Average population density as a function of radial distance to volcanoes in Significant Volcanic Eruption Database (SV), in 1975, 1990, 2000, and 2015.

Differently from some previous analyses (e.g., Small and Naumann 2001), we have considered all volcanoes listed in the databases regardless of their type or location, as e.g., submarine volcanoes may still affect populations on land upon erupting, as many are located in proximity to inhabited areas. This is especially relevant for remote archipelagos (such as Tonga, French Polynesia, 
Solomon Islands Guam, Hawaii, Mariana Islands), where potential exposure and risk is typically overlooked in global analyses and where DRM poses specific challenges. In fact, we think this may be a 'hidden' source of potential exposure and risk that should deserve more attention in future research. Of 118 submarine volcanoes in the HV list, 76 have documented eruptions.

Figure 32 and Figure 33 show the average population densities (over land) by radial distance to volcanoes. These charts show that relatively high population densities occur in the vicinity of all volcanoes, especially of those with significant eruptions (above the overall global densities over land, which are 27 and 49 people/km2 in 1975 and 2015, respectively); and these densities increase sharply away from volcanoes but in very close proximity, especially from SV, in whose vicinity densities are significantly higher. Moreover, while maintaining the overall pattern, those densities have been increasing considerably since 1975, particularly in the period 1975-1990. However, it is at a distance of 10 to $20 \mathrm{~km}$ from Holocene volcanoes that the absolute increase in population density has been greatest (additional 75 persons/km2), from 1975 to 2015.

In all periods, population density generally increases with close proximity to volcanoes, peaking at around 15-20 km away, and then decreases sharply in their immediate vicinity, but still maintaining relatively high densities. This sharp decrease may be due to presence of steep slopes, volcanic activity, or enforcing of exclusion zones for settlement. This pattern is even more striking for volcanoes with significant eruptions (SV), where overall population densities are higher (up to 270 people/km2), with obvious implications for exposure, risk analyses, and potential impacts. This pattern is dissimilar from the monotonically decreasing densities reported by Small and Naumann 2001. This difference may be the outcome of sharper analyses enabled by the characteristics of the new population grids, i.e., effective disaggregation to built-up areas and higher spatial detail.

These results further detail, revise and update previous estimates, such as that of UNISDR 2015b that states "... at present, more than 800 million people in 86 countries live within $100 \mathrm{~km}$ of a volcano that could potentially erupt (GVM, 2014a)", and that "... over 29 million people worldwide live within just $10 \mathrm{~km}$ of active volcanoes, and around 800 million people live within 100 km" (Brown et al. 2015). Likely reasons for such disagreement include their reliance on conceptually different population grids (e.g., LandScan versus GHS-POP), and their reliance on older census data. Also, LandScan does not depart from or aim to model distribution of residential population, from which population living in proximity can be estimated with more confidence, but instead maps ambient population. 


\subsubsection{Sources of Uncertainty}

As with all global data, both the volcano datasets and the population grids are affected by error and uncertainty. This analysis is performed using a $250 \mathrm{~m}$ population grid, which also makes it sensitive to uncertainty in spatial precision. The uncertainties in GHS-POP mostly result from the quality and reliability of censuses and the capacity to fully detect built-up in diverse landscapes using heterogeneous Landsat image archives (Pesaresi et al. 2016). Such issues typically worsen with temporal distance from the current period (for a more detailed discussion, see reference (Freire et al. 2018a). Despite the mitigation procedures implemented in the production of the current population grids, some issues remain unsolved in global population data, which should not be used for pixel level assessments.

Issues of spatial accuracy and precision also affect the GVP datasets in which volcanoes are represented through points (coordinate pairs), and a single point frequently refers to a group of volcanoes or craters (e.g., volcanic fields) or giant calderas (e.g., Yellowstone, USA); for some of these, due to their size and extent, an areal feature (polygon) would be a better representation for the potential hazard source. Also, the geographical coordinates (decimal degrees) are often coarse with precision sometimes limited to the first decimal, which corresponds to a real world precision of about $5.5 \mathrm{~km}$. This is clearly insufficient for detailed analyses in close proximity to volcanoes. This vagueness and low spatial precision reduce accuracy and discourage more detailed analyses. These and other uncertainties introduce limitations for studies: while these are usually mitigated when aggregated assessments such as this one are conducted, they can become apparent when analyses are carried out for individual volcanoes or at very fine scale (e.g., very close proximity).

\subsubsection{Population Distribution from 1975 to 2015 in Relation to Volcanism, in Southeast Asia and Central America}

This section analyzes in more detail results for two important volcanic regions, Southeast Asia and Central America. The first region comprises the countries of Indonesia, Philippines, and East Timor, while the second includes Mexico, Guatemala, Belize, Honduras, El Salvador, Nicaragua, Costa Rica, and Panama. Figure 34 shows location of Holocene volcanoes in these regions and the selected distances under analysis. SE Asia and Central America together represent $40 \%$ of the total global population living within $100 \mathrm{~km}$ of volcanoes, but with different contributions and patterns of population by radial distances (Table 20). 

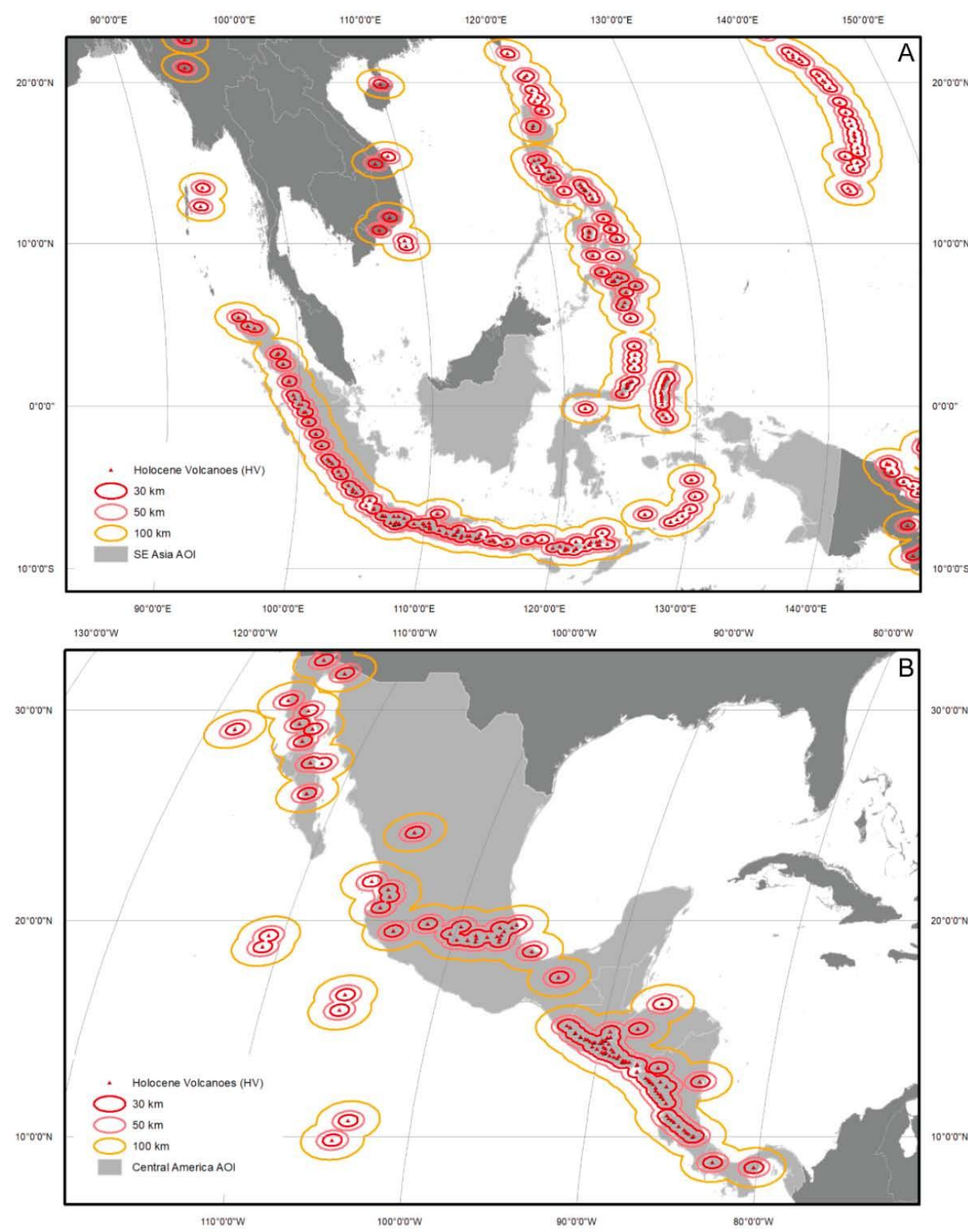

Figure 34 Selected distances from Holocene volcanoes in (A) Southeast Asia and (B) Central America.

Table 20 Population (in millions and percentage of region total) by radial distance of Holocene volcanoes (HV) and volcanoes in Significant Volcanic 
Eruption Database (SV) in 1975, 1990, 2000, 2015, in Southeast Asia and Central America.

\begin{tabular}{|c|c|c|c|c|c|c|c|c|}
\hline & \multicolumn{2}{|c|}{1975} & \multicolumn{2}{|c|}{1990} & \multicolumn{2}{|c|}{2000} & \multicolumn{2}{|c|}{2015} \\
\hline $\begin{array}{l}\text { Dist. } \\
\text { (km) }\end{array}$ & HV & SV & HV & Sv & HV & SV & HV & SV \\
\hline \multicolumn{9}{|c|}{ Southeast Asia } \\
\hline \multirow{4}{*}{10} & 11 & {$[4$} & 18 & {$[7$} & 22 & {$[8$} & 27 & {$[10$} \\
\hline & $(6.6 \%$ & $(2.5 \%)$ & $(7.3 \%$ & $(2.8 \%)$ & $(7.5 \%$ & $(2.9 \%)$ & $(7.4 \%$ & $(2.9 \%)$ \\
\hline & ) & & ) & ] & ) & & ) & ] \\
\hline & 73 & {$[42$} & 109 & {$[59$} & 129 & {$[69$} & 153 & {$[81$} \\
\hline \multirow{3}{*}{30} & $(42.5$ & $(24.2 \%$ & $(44.6$ & $(24.3 \%$ & $(44.3$ & $(23.7 \%$ & $(42.5$ & (22.4\% \\
\hline & $\%)$ & )] & $\%)$ & )] & \%) & )] & $\%)$ & )] \\
\hline & 118 & {$[80$} & 171 & [113 & 202 & {$[132$} & 243 & {$[157$} \\
\hline \multirow[t]{2}{*}{50} & $\begin{array}{c}(68.4 \\
\%)\end{array}$ & $\begin{array}{c}(46.2 \% \\
)\end{array}$ & $\begin{array}{c}(69.9 \\
\%)\end{array}$ & $\begin{array}{c}(46.1 \% \\
)\end{array}$ & $\begin{array}{c}(69.6 \\
\%)\end{array}$ & $\begin{array}{c}(45.4 \% \\
)]\end{array}$ & $\begin{array}{c}(67.7 \\
\%)\end{array}$ & $(43.6 \%$ \\
\hline & 143 & {$[132$} & 207 & [189 & 247 & [225 & 301 & {$[272$} \\
\hline \multirow[t]{2}{*}{100} & $(82.8$ & $(76.2 \%$ & $(84.9$ & $(77.4 \%$ & $(85.1$ & $(77.4 \%$ & $(83.7$ & $(75.8 \%$ \\
\hline & $\%)$ & )] & $\%)$ & )] & $\%)$ & )] & $\%)$ & )$]$ \\
\hline \multirow{2}{*}{$\begin{array}{c}\text { Regi } \\
\text { on }\end{array}$} & \multicolumn{2}{|c|}{$173(100 \%)$} & \multicolumn{2}{|c|}{$244(100 \%)$} & \multicolumn{2}{|c|}{$290(100 \%)$} & \multicolumn{2}{|c|}{359 (100\%) } \\
\hline & & & \multicolumn{4}{|c|}{ Central America } & & \\
\hline \multirow{3}{*}{10} & 6 & {$[2$} & 8 & $\begin{array}{l}{[3} \\
70 \%)\end{array}$ & 9 & {$\left[\begin{array}{c}3 \\
5 \%\end{array}\right.$} & 11 & {$[4$} \\
\hline & $\begin{array}{c}(7.1 \% \\
)\end{array}$ & $(3 \%)]$ & ) & $\begin{array}{c}(2.7 \%) \\
]\end{array}$ & $\begin{array}{l}5 \% \\
\text { ) }\end{array}$ & $\begin{array}{c}(2.5 \%) \\
]\end{array}$ & $\begin{array}{l}5.4 \% \\
)\end{array}$ & $\begin{array}{l}.3 \%) \\
]\end{array}$ \\
\hline & 29 & {$[11$} & 40 & {$[16$} & 47 & {$[19$} & 57 & {$[23$} \\
\hline \multirow[t]{3}{*}{30} & $(35.3$ & $(14.1 \%$ & $(34.6$ & $(13.9 \%$ & (34.1 & $(13.8 \%$ & $(32.8$ & $(13.4 \%$ \\
\hline & $\%)$ & )$]$ & $\%)$ & )$]$ & $\%)$ & )$]$ & $\%)$ & )$]$ \\
\hline & 41 & [22 & 56 & {$[30$} & 67 & {$[36$} & 81 & {$[43$} \\
\hline \multirow[t]{2}{*}{50} & $\begin{array}{c}(50.3 \\
\%)\end{array}$ & $(27 \%)]$ & $\begin{array}{c}(49.1 \\
\%)\end{array}$ & $\begin{array}{c}(26.4 \% \\
)]\end{array}$ & $\begin{array}{c}(48.3 \\
\%)\end{array}$ & $\begin{array}{c}(25.9 \% \\
)]\end{array}$ & $\begin{array}{c}(46.7 \\
\%)\end{array}$ & $\begin{array}{c}(24.9 \% \\
\text { )] }\end{array}$ \\
\hline & 57 & {$[44$} & 81 & [60 & 97 & {$[71$} & 120 & {$[86$} \\
\hline \multirow[t]{2}{*}{100} & $(70.6$ & (53.9\% & $(70.3$ & $(52.2 \%$ & $(70 \%)$ & $(51.2 \%$ & $(69.4$ & $(49.7 \%$ \\
\hline & \%) & )] & $\%)$ & )] & & )] & \%) & )] \\
\hline $\begin{array}{c}\text { Regi } \\
\text { on }\end{array}$ & \multicolumn{2}{|c|}{$81(100 \%)$} & \multicolumn{2}{|c|}{$115(100 \%)$} & \multicolumn{2}{|c|}{139 (100\%) } & \multicolumn{2}{|c|}{$173(100 \%)$} \\
\hline
\end{tabular}

In SE Asia the population within $100 \mathrm{~km}$ of $\mathrm{HV}$ has increased from 143 million in 1975 to 301 million in 2015, representing 84\% of the region's inhabitants. In Central America the population within this distance increased from 57 million in 1975 to 120 million in 2015, comprising 69\% of the region's inhabitants. Especially striking in SE Asia are the 10 and the 27 million people currently living within $10 \mathrm{~km}$ of SV and HV, respectively, accounting for almost half of the global population within this range of recently active volcanoes. This short distance is especially problematic in small inhabited islands which are completely contained within this range, where fast evacuation can be particularly challenging.

However, while in SE Asia the share of total population within $100 \mathrm{~km}$ of HV has increased (albeit slightly) between 1975 and 2000, in Central America this value has been decreasing, and even more so when SVs are considered. 
Concerning the annual growth rates of population by radial distance of HV and SV, Table 21 shows important differences between and within these regions.

Table 21 Compound annual growth rate of population by radial distance of Holocene volcanoes (HV) and volcanoes in the Significant Volcanic Eruption Database (SV), for each time interval, in Southeast Asia and Central America.

\begin{tabular}{|c|c|c|c|c|c|c|c|c|}
\hline & \multicolumn{2}{|c|}{$1975-1990$} & \multicolumn{2}{|c|}{ 1990-2000 } & \multicolumn{2}{|c|}{ 2000-2015 } & \multicolumn{2}{|c|}{$1975-2015$} \\
\hline Dist. (km) & HV & SV & HV & SV & HV & SV & HV & SV \\
\hline \multicolumn{9}{|c|}{ Southeast Asia } \\
\hline 10 & 3.05 & [3.15] & 2.10 & [2.16] & 1.36 & [1.34] & .17 & {$[2.22]$} \\
\hline 30 & 2. & {$[2$.} & 1.69 & & & & 1. & {$[1.65]$} \\
\hline 50 & 2.48 & {$[2$.} & 1.7 & & 1.25 & {$[1$.} & 1.82 & {$[1.7]$} \\
\hline 100 & & {$[2$.} & 1.77 & {$[1.74]$} & 1.32 & {$[1$.} & 1.88 & {$[1.83]$} \\
\hline Regior & \multicolumn{2}{|c|}{2.34} & \multicolumn{3}{|c|}{$\begin{array}{l}1.75 \\
\text { ral America }\end{array}$} & .43 & \multicolumn{2}{|c|}{1.85} \\
\hline 10 & 2.14 & [1.67] & 1.67 & [1.31] & 1.07 & {$[0.84]$} & 1.62 & {$[1.27]$} \\
\hline 30 & 2.21 & {$[2.25]$} & 1.75 & {$[1.84]$} & 1.21 & {$[1.28]$} & 1.72 & {$[1.78]$} \\
\hline 50 & 2.19 & [2.19] & 1.74 & {$[1.74]$} & 1.24 & [1.19] & 1.72 & {$[1.7]$} \\
\hline 100 & 2.31 & [2.13] & 1.88 & {$[1.71]$} & 1.41 & [1.27] & 1.86 & [1.7] \\
\hline Region & \multicolumn{2}{|c|}{2.34} & \multicolumn{2}{|c|}{1.91} & \multicolumn{2}{|c|}{1.47} & \multicolumn{2}{|c|}{1.91} \\
\hline
\end{tabular}

While in SE Asia the highest growth rates have occurred in close proximity to HV and SV volcanoes (within $10 \mathrm{~km}$ ), in Central America the strongest rates are observed farther away (beyond $50 \mathrm{~km}$ ), especially after 1990 and for HV. Also, whereas in SE Asia much of this growth has occurred at rates well above the regional ones between 1975 and 2000 (especially for HV), in Central America increases stayed below regional rates for all distances and periods, suggesting that areas more distant from volcanoes were favored for settlement. While explaining the observed variations is beyond the scope of this paper, we can offer some hypotheses for the underlying causes:

(a) Pressure for space in a context of relatively weak planning, causing settlement to encroach on volcanoes (overall average population densities are much higher in SE Asia than in Central America);

(b) More recent dates of eruptions and variations in risk perception.

Additional research is needed to test these and other hypotheses that may explain settlement patterns.

Regarding variation of population densities in these regions in proximity of HV and SV, Figure 35 and Figure 36 show some differences. For both volcano sets, population densities are generally higher in SE Asia, especially in 2015 where these can be more than twice those in Central America (HV). In SE Asia, population densities increase faster with distance from $\mathrm{HV}$ and remain relatively high ( $>400$ persons $/ \mathrm{km} 2$ ) up to $44 \mathrm{~km}$ away. Beyond $4 \mathrm{~km}$, density surpasses the overall mean for the region (78 and 163 persons $/ \mathrm{km} 2$ in 1975 and 2015, respectively). There is a pronounced peak in densities around a distance of $18-22 \mathrm{~km}$ that has intensified since 1975. Because this is 
essentially a mountainous landscape, this may also reflect the average distance of valley floors (or the mid-distance between consecutive mountains), where conditions for settlement are more favorable. Figure 37 illustrates the situation in 2015 in the Island of Java (Indonesia), showing the locations of Holocene volcanoes, main cities, and the population distribution. 


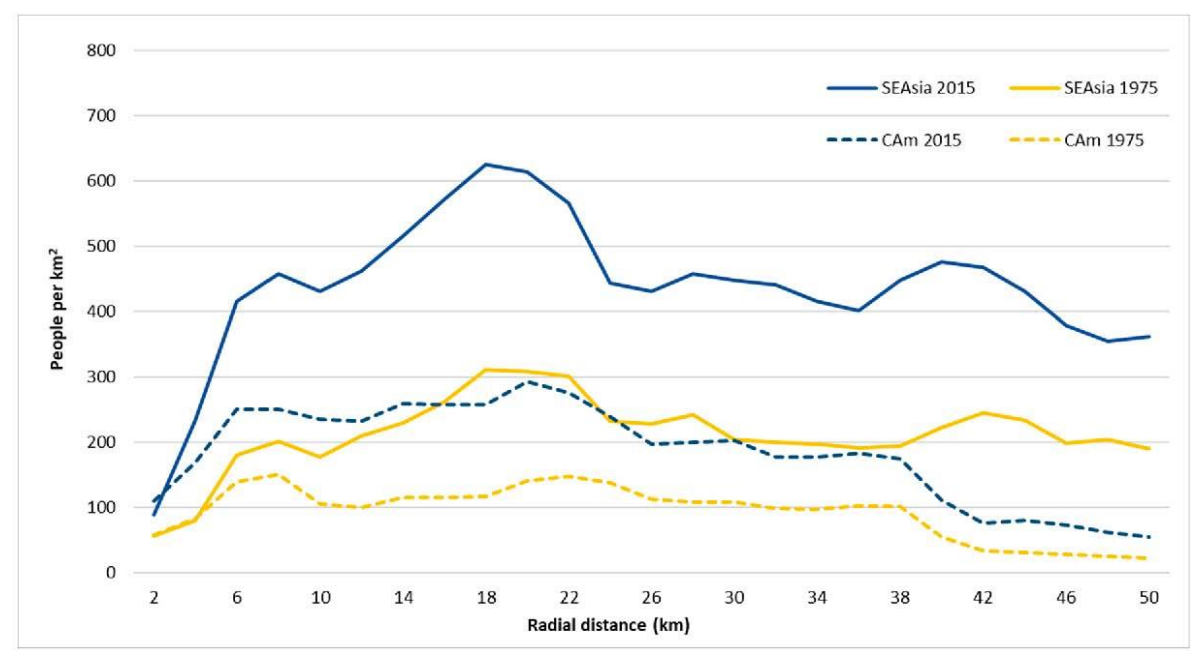

Figure 35 Average population density in Southeast Asia (SEAsia) and Central America (CAm) as a function of radial distance of Holocene Volcanoes (HV), in 1975 and 2015.

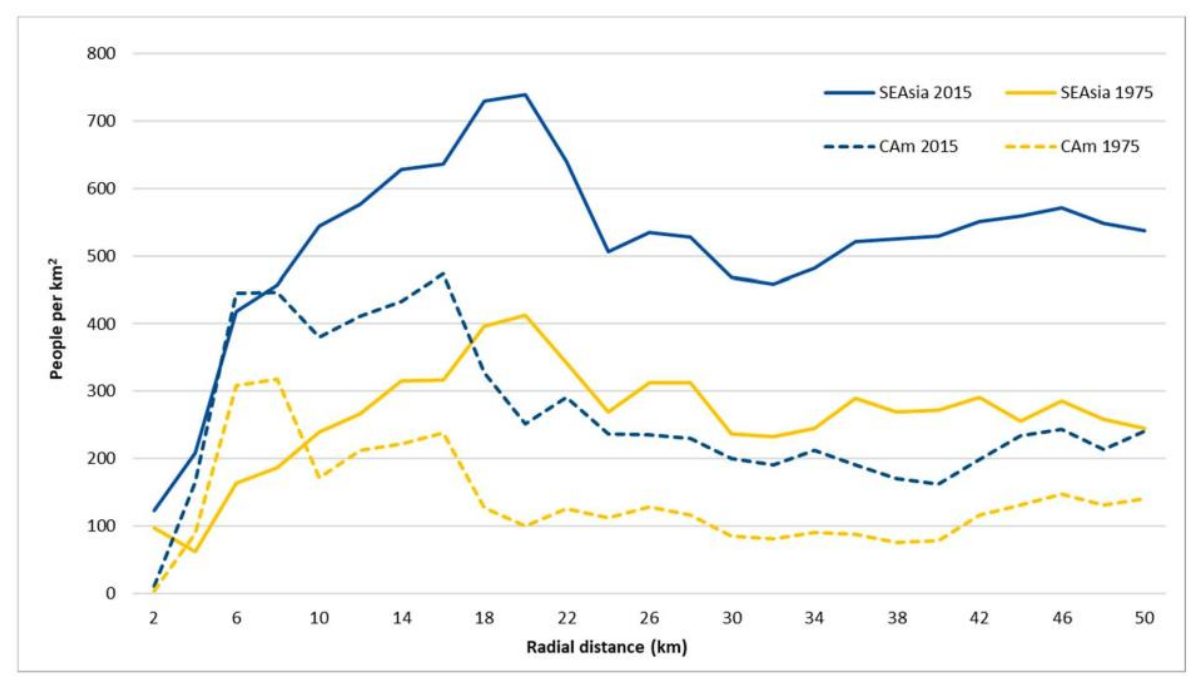

Figure 36 Average population density in Southeast Asia (SEAsia) and Central America (CAm) as a function of radial distance of volcanoes in Significant Volcanic Eruption Database (SV), in 1975 and 2015. 


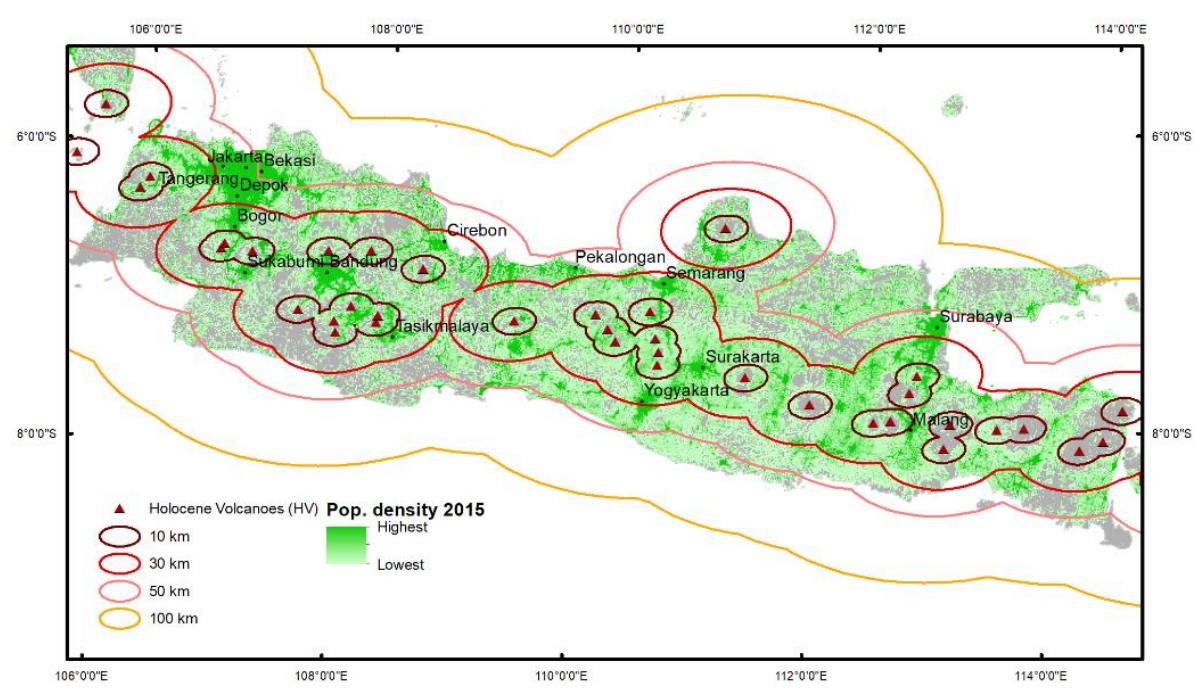

Figure 37 Island of Java (Indonesia), showing selected distances from Holocene volcanoes, main cities, and population distribution in 2015 from GHS-POP (World Mollweide projection).

Regarding SV volcanoes (Figure 36), in Central America densities increase dramatically up to 6-8 $\mathrm{km}$ and remain high up to $16 \mathrm{~km}$, decreasing markedly after that distance. SE Asia displays a similar proximal pattern for both HV and SV, with very high densities observed around $20 \mathrm{~km}$ from SV (700 people/km2) and remaining high until $50 \mathrm{~km}$ away. These results suggest that the overall settlement patterns observed, although specific for each region, were mostly defined prior to 1975, especially in Central America. These spatio-temporal patterns of settlement have consequences for DRM. This analysis confirms and characterizes increases in the exposure of population. Due to the large size, densities, and trends of population potentially exposed to volcanic activity, coupled with structural vulnerabilities, SE Asia and Central America constitute hot spots of exposure and risk. In both regions, the high densities of inhabitants in the immediate vicinity of volcanoes poses widespread challenges for DRM, in particular for evacuation though also the sheer scale of response and recovery operations can become quite daunting in case of large eruptions. In this context, effective monitoring of volcanic activity, emergency preparation and early warning can significantly reduce local risk and potential impacts from eruptions. Furthermore, these new population data and results have implications for exposure indices, such as the VPI (Ewert et al. 2004) and PEI (Aspinall et al. 2011). Due to their reliance on and sensitivity to population distribution data, such indices should use the latest population grids available and be regularly updated to accurately reflect the current exposure and risk situation. 


\subsection{Conclusions}

Where human population grows there is an increasing pressure to settle on more hazardous areas, including in areas subject to volcanic activity. Active volcanoes generate diverse hazardous phenomena which can affect populations in their immediate vicinity but also farther away. Volcanic eruptions are not yet influenced by human activity nor can their time, location, or magnitude be mitigated by human capacity and ingenuity. Therefore, the available approaches for effective DRR are risk estimation, monitoring, awareness raising, implementation of impact mitigation measures, and preparation for events. These require detailed knowledge of potential human exposure through improved geoinformation on population distribution and densities, in space and time. As a contribution to this effort, we have characterized globally the geographical distribution of human population with proximity to historically active volcanoes, and its recent evolution from 1975 to 2015 . We have combined recently produced detailed and multi-temporal geospatial grids of global population densities with the latest data on distribution of Holocene volcanoes and those with evidence of significant eruptions. This analysis updates and refines earlier estimates and introduces a previously unavailable spatio-temporal dimension to analysis of human settlement in proximity of volcanoes.

We find that population is not evenly distributed across geographical space around volcanoes and that concentrations have been increasing since 1975 . Results concerning the overall global situation indicate that in 2015 more than 600 million people ( $8.4 \%$ of total) lived within $100 \mathrm{~km}$ of a volcano with at least one significant eruption, and more than 1 billion people (14.3\% of the total) within that distance of a Holocene volcano. This last amount has increased by $1.57 \%$ per year since 1975 , above the global population growth rate for the period. Especially significant have been the growth rates and total population living within $10 \mathrm{~km}$, which in 2015 totals 59 million for Holocene volcanoes and 21 million for those with significant eruptions. Almost half of this population lives in Southeast Asia. Population densities in vicinity of volcanoes are also higher than global average densities, and those have been increasing with time in last 40 years. We find that overall spatial patterns have been relatively stable in time but that their variation with distance is not monotonic, with density generally increasing with proximity to volcanoes, peaking at around 15-20 km away, and then decreasing sharply in their immediate vicinity.

Despite both regions having high potential exposure to volcanoes, we find differences between Southeast Asia and Central America: in SE Asia the share of total population within $100 \mathrm{~km}$ of HV has increased (albeit slightly) between 1975 and 2000, and the highest growth rates occur in close proximity to HV and SV volcanoes (within $10 \mathrm{~km}$ ); in Central America the share of population within $100 \mathrm{~km}$ of HV and SV has been decreasing, and the strongest rates are 
observed farther away from volcanoes. In inhabitable land, especially in fertile tropical climates, volcanoes seem to have exerted a noticeable 'pull-and-push' effect on humans that contributed to determining the specific settlement patterns we currently observe.

Not only do these more detailed and comprehensive analyses update and revise earlier estimates and findings, but they also enable monitoring of patterns and detection of changes with time. This work also illustrates how emerging open and free geospatial datasets can narrow gaps in population distribution data and knowledge for supporting Disaster Risk Management and Reduction activities. In this domain, these new population grids could benefit a global volcano warning system, by improving estimation of potential human impacts (Andredakis et al. 2015). In case of volcanic eruption preparedness, such geo-information could be used for baseline evacuation planning and estimation of resources needed for adequate response. While this work has focused on volcanism, these population grids can also be combined with any type of hazard, enabling improved modeling and analyses throughout the emergency management cycle.

Author Contributions: Conceptualization, Sergio Freire, Aneta J. Florczyk, and Martino Pesaresi; Data curation, Aneta J. Florczyk; Formal analysis, Sergio Freire and Aneta J. Florczyk; Methodology, Sergio Freire and Aneta J. Florczyk; Writing-original draft, Sergio Freire and Aneta J. Florczyk; Writing-review \& editing, Sergio Freire, Richard Sliuzas.

Acknowledgments: The authors would like to thank Michele Melchiorri and Marcello Schiavina for their technical and moral support. We thank the three anonymous reviewers for their comments and suggestions. 
Chapter 7. Synthesis, conclusions, and outlook 


\subsection{Introduction}

This chapter summarizes the main contributions of the approaches presented in previous chapters, including challenges, and discusses ongoing and future developments in modeling population distribution to support Disaster Risk Management and Reduction.

The main objective of this thesis was to develop methods to improve the mapping of population distribution at a range of spatial and temporal scales for benefiting analyses of population exposure and related DRM activities.

\subsection{Main results: Modeling population distribution in space and time to support Disaster Risk Management}

This $\mathrm{PhD}$ research involved addressing challenges in modeling, scaling-up approaches, data availability and quality. In brief, the main points and contributions of this thesis are the following:

1) Since impacts of hazards and disasters are place and time dependent, several DRM and DRR phases and activities would benefit from relying on more spatially-detailed and time-specific assessments of population exposure, at a range of relevant spatio-temporal scales (local to global).

2) Improving population distribution data for human exposure assessment requires addressing challenges present in input data and geospatial modeling. While at local scale in data rich environments more detailed and sophisticated models can be developed with acceptable uncertainty, scaling up such approaches to the global domain requires addressing different challenges while accepting some limitations ${ }^{39}$ in data availability, quality, and concepts so as not to compromise policy support and maximize the range of uses of population data, especially for supporting International Frameworks.

3) Geospatial information on population distribution constitutes crucial baseline data for risk analysis and DRM across a range of hazards and threats, and

${ }^{39}$ An example of such concessions is the fact that GHS-POP currently allocates resident population to all mapped built-up areas (buildings) located within populated source zones from census, and therefore also to those that lack residential function. This is a contingency imposed by lack of consistent and complete worldwide mapping of functional use of buildings. In practice, in populated units that contain non-residential buildings (i.e. horizontal mixeduse), GHS-POP allocates some population counts to these buildings while subtracting them from residential ones (ensuring volume preservation). In this respect, it can be argued that GHS-POP approaches a more spatially-restricted representation of 'ambient' population (as LandScan) where 'ambient' population is constrained to buildings. However, in cases where a single population grid is produced such representation might be more suitable for certain DRM applications, as confirmed by INFORM (http://www.informindex.org). 
investing in improving data benefits exposure assessment by detailing and revealing a sharper picture, with the aim of 'leaving no one behind'; improved population data are simultaneously relevant and generic as to enable improved modeling and analyses throughout the emergency management cycle and in all domains where the spatio-temporal distribution of people is pertinent. This last point is illustrated in the next section (7.3), showing applications of the produced population distribution data in diverse domains and for different purposes.

Below we summarize the main contributions and findings of each chapter, considering their specific research objectives and questions, as well as strengths and weaknesses of the approach followed.

\subsubsection{Chapter 2: Modeling of spatio-temporal distribution of urban population at high-resolution - value for risk assessment and emergency management}

Chapter 2 addressed two main questions:

(i) Is the spatial distribution of urban population in the daily cycle relevant for risk analysis (exposure) at local scale?, and

(ii) How can more detailed modeling of spatio-temporal distribution of urban population contribute to DRM, considering its cycle?

The research has shown that at local scale there is significant variation of population in the daily cycle, and the quantification of variation in population exposure to different hazard types, natural and man-made, demonstrated the relevance the new data makes for sharpening risk analyses. We have illustrated for different DRM scenarios that use of census data (resident population) can cause significant errors in the estimates of potentially affected population due to daytime events, suggesting the temporal matching of population exposure data to the hazard event. With this knowledge, the chapter provides a first known attempt at systematizing and suggesting contributions of detailed population distribution data for decision support according to main phases of the DRM cycle: planning, mitigation, preparedness, response, and recovery.

The population model includes several limitations and assumptions. These include its relatively stringent data requirements, and the use of what is usually a commercial input data set (the workplaces and employment database) -despite coming from a public source. The fact that data is neither open nor free constrains their accessibility and applications. The geocoding of workplaces' specific street addresses makes the model more spatially accurate and precise, but this is a time-consuming task that increases cost of final data production. 
Moreover, the concept of workplace ${ }^{40}$ limits correct assignment of workers in spatially 'distributed activities' such as cleaning and security services.

Due to the absence of information on building volume or floor space, population counts from source zones were disaggregated to residential streets (target zones) using areal weighting. Also one single parameter per municipality was available to derive daytime residential population for a workday, and the assumption that all workers are in their workplaces and non-commuters remain in their residences in the daytime period is obviously a simplification of a more complex reality. In the frame of DRM applications and scenarios discussed, modeling of outdoor population would be especially relevant and valuable for scenarios A-technological hazard (airborne toxic plume release) and Dplanning of best route for transportation of hazardous materials.

On the positive side, the consistency among input data sets - especially temporal currency - was quite high, which becomes increasingly difficult as the geographical scale (scope) of models expands. The adopted modeling approach supports local-level analysis, while also efficiently accommodating people that work at home, by not considering that all active population leaves their residences during the workday. In addition to surfaces of nighttime (residential) and daytime population, this approach yields detailed grids of workers/students and daytime residential population, that can be useful in DRM applications. Also, an 'ambient' population grid can be derived in a transparent way by computing a weighted average of nighttime and daytime distributions. Consideration of spatio-temporal population distribution in this context has allowed two fundamental issues to be addressed simultaneously in scenario $D$ : what is the best period (day or night) and route in order to minimize population exposure along the way?

The main value of these results includes the increased spatial resolution of residential ( n nighttime) distribution, the fact that both nighttime and daytime distributions share the same spatial reference basis, and that daytime distribution and potential population exposure is better estimated than was previously possible using residential population.

\subsubsection{Chapter 3: Integrating population dynamics into mapping human exposure to seismic hazard}

Chapter 3 sought to answer two questions:

(i) Can new geospatial data on spatio-temporal distribution of population, derived by scaling-up a costly local-based approach, be combined with an

${ }^{40}$ In the workplace database all workers are assigned to the locations of the employing companies/services, although their activities may be conducted elsewhere (e.g. professional drivers). 
earthquake hazard map to improve human exposure mapping and assessment in a metropolitan area?, and

(ii) What is the estimated variation in population exposure by seismic level in the Lisbon Metropolitan Area (LMA) in day- and nighttime periods?

Regarding the first question, in this chapter an approach was developed that enables modeling and mapping of spatio-temporal population distribution and density in the daily cycle at high spatial resolution for Metro Areas in Portugal. The day- and nighttime population grids were produced for the Lisbon Metro Area (LMA) by scaling-up a previous local-based approach (presented and applied in chapter 2). The population model generated a nighttime (resident) raster distribution at a higher resolution than census data and a comparable daytime population surface, previously unavailable for the study area. The refined spatio-temporal population surfaces were successfully combined with a recent seismic intensity map to map and estimate population exposure in the daily cycle and derive new spatial representations of this exposure, i.e. spatially-explicit four-class-composites of human exposure to seismic intensity. Results show that, although in the standard approach very little of the populated area is classified as Very High exposure, this zone includes almost a quarter of the daytime population in the LMA. The work confirms that population exposure analysis based on census data alone may result in significant underestimates of risk for a daytime event. Furthermore, it has shown that the introduced spatio-temporal refinements in modeling population densities enabled a more thorough assessment of potential human exposure in the LMA.

Concerning the second question, the analysis of population exposure to seismic levels in the LMA showed that there are considerable differences from nighttime to daytime, with significantly more people potentially at risk in the daytime period. Results indicate that from night to day the population exposed to the highest seismic level (i.e. level IX) increases by $22 \%$ to affect $5 \%$ of the total daytime population of LMA (137,222 people). Even more important, from the nighttime to daytime period an additional 204,786 persons are estimated to be exposed to the levels VIII and IX, whose zones then contain $52 \%$ of the daytime population.

Regarding shortcomings of this work, sources of uncertainties in population model are in the spatial nature and dynamic character of activities used to produce the daytime worker and student population grid, combined with the unavailability of mobility statistics more detailed than the municipal level. Quality assessment of the workforce grid in the test area revealed modest correlation with detailed reference data. This may be caused by errors or inconsistences in mapping "labor" classes in the available land use maps (i.e. Industrial, Commercial, and Service areas), combined with lack of indication of workers' density in these 2-D land use patches, and inaccuracies generated by 
disaggregating the population based on the raster surface of the street network.

Turning to the strengths of this work, the requirements and availability of input data sets would allow extending the population modeling approach to the Oporto Metropolitan Area, further encompassing nine municipalities and $1,759,524$ inhabitants (2011). The proposed modeling approach proved to be sustainable (repeatable) for these areas as required input data matching the 2011 census was made available and even improved, now covering the whole country of Portugal. Due to their characteristics, such population distribution datasets can be combined with different hazard maps to improve spatiotemporal exposure assessment and mapping for any type of hazards, as shown in chapter 4 with tsunami. In the case of seismic risk, potentially the detailed population surfaces could be used as input in earthquake simulators for modeling of human casualties. The approach to mapping and assessing human exposure can be applied to other areas where similar data is available, highlighting hotspots of exposure to seismic hazard or other threats where population density is a relevant measure of vulnerability, and may assist in prioritizing areas for mitigation, preparedness and response actions.

\subsubsection{Chapter 4: Advancing tsunami risk assessment by improving spatio-temporal population exposure and evacuation modeling}

The main question related to this chapter was:

How is the spatio-temporal distribution of population in the daily cycle in a metropolitan area relevant for population exposure to and evacuation from tsunami hazard?

The research considers the spatio-temporal population distribution in assessing risk of tsunami in a metropolitan area. Considering the variation of population distribution in the daily cycle at high spatial resolution, both the potential population exposure in each of those periods and speed of evacuation were modeled and analyzed. Results show that potential population exposure to tsunami is significantly time-dependent in the metropolitan area if commuting dynamics are considered, displaying a strong variation from nighttime to daytime, thus confirming the importance of accounting for human dynamics in this cycle for DRM and reduction of impacts.

In case of tsunami, the analysis indicates that a significant amount of population is potentially at risk, and its numbers increase dramatically from nighttime to daytime, especially in the zones of high hazard susceptibility. Due to concentration of economic activities and public and private services in the coastal hazard zone, the overall potential exposure doubles from nighttime to daytime, representing $16 \%$ of the population of the Lisbon Metropolitan Area. In areas of high hazard susceptibility to tsunami, population movement results in Daytime population being 2.5 times the number of residents. Evacuation 
modeling shows significant differences in the magnitude and speed of evacuation between night and day, and how this may be affected by varying population density. Analysis reveals that full horizontal evacuation may be problematic in the daytime period, even if initiated after a potential tsunamitriggering earthquake. This finding suggests that the consideration of alternatives for preparedness measures, such as vertical evacuation, should be considered.

Main weaknesses of this work include the limitations and assumptions of the population distribution model, already mentioned in chapter 3, despite efforts undertaken to improve the quality of population grids. Estimation of potential population exposure and evacuation is based on a worst-case scenario, as the return period for hazard event was not available. In a large city and metropolitan area, modeling exposure in 2-D is likely to lead to over-estimation of potential impacts (including human) as many tall buildings and structures may afford greater protection from direct tsunami impacts ${ }^{41}$. For full assessment of tsunami risk, vulnerability of exposed elements should also be considered, especially in the absence of early warning. Structural vulnerabilities of buildings are particularly relevant in case of nighttime event when most people can be expected to be in their homes sleeping.

Strong aspects of this work are the modeling of population and assessment of exposure at high spatio-temporal resolution. Tsunamis are time-specific, sudden hazard events whose risk management activities have a lot to benefit from availability of population models in daily cycle for planning, mitigation, preparedness, and response. Modeling population at very high spatial resolution is also important due to nature of water as fluid, which enables it to affect very specific and small areas.

In addition to providing valuable information for planning and managing evacuation measures, the work suggests subsequent developments. These include conducting a more detailed spatial modeling of population distribution and exposure assessment to tsunami at the building level considering their characteristics and enabling modeling of vertical evacuation. A demonstration of detailed spatial modeling of population distribution and exposure assessment to tsunami at the building level considering its function, height and elevation for part of the same study area (Lisbon, Portugal) was carried out by Freire et al. 2011a. Building further on this work, feasibility of vertical evacuation was modeled and assessed for same three communes of the city of Lisbon (Freire et al. 2012), identifying 165 potential shelters for vertical evacuation and estimating that all exposed residents are able to evacuate to safety after 10 minutes if this alternative is considered.

41 This issue was addressed in subsequent work where 3-D modeling of exposure was undertaken: Freire et al. 2012. 


\subsubsection{Chapter 5: Enhanced data and methods for improving open and free global population grids: putting 'leaving no one behind' into practice}

Chapter 5 sought to answer the question:

How can some major shortcomings in geospatial population statistics be detected, assessed, and mitigated with remote sensing information and automated approaches, while preserving the statistic's integrity for policy making?

The research shows the value of detailed, updated, and independent global remote sensing information to complement and improve conventional sources of fundamental population statistics. New automated procedures to detect and mitigate major discrepancies and anomalies occurring in geospatial census data were developed, tested and implemented. Global and consistently-derived remote sensing information reporting on built-up presence from the Global Human Settlement Layer (GHSL) was used to revise census units deemed as 'unpopulated' and to harmonize population distribution along coastlines, while minimizing changes to the original population data. To accomplish this, an automated method was proposed and applied: the 'split \& merge' approach.

Respecting the overall integrity of statistics is important, and is often a needed compromise to ensure its 'acceptance' for policy support by national institutional stakeholders. This mitigation approach illustrates the existing tension between producing the best data possible vs. using official data that is accepted by stakeholders (e.g. national authorities) as base for monitoring and informing policy in international agreements (e.g. SDGs). Results show that the targeted anomalies were significantly mitigated and that the baseline census database has improved, potentially benefitting other uses of the same statistical base. Outcome and benefits for population disaggregation were illustrated.

Strengths of this approach are its use of open and free information from remote sensing, and the automated approach for detection and mitigation of anomalies, while harmonizing both data sets. Relying on open and free information and on an automated approach benefits transparency, repeatability and cost-effectiveness (i.e. sustainability) of procedures. The developed 'split and merge' approach proved effective at mitigating both types of detected anomalies, while minimizing changes to census geometry, preserving the regional distribution of population, and the overall counts at the upper administrative level. Another positive outcome is the improved representation of the process of settlement expansion through land reclamation from the sea, and accounting for human settlements extending over water bodies such as floating settlements. 
In context of risk analysis and DRM activities, this contributes to improving the accuracy of exposure assessment in coastal areas, especially those more susceptible to sea level rise and coastal hazards (tsunamis, hurricanes/cyclones, storm surge). These grids were already used for assessing the proportion of urban residents and urban land less than $10 \mathrm{~m}$ above sea level (CUT 2019).

Still several of limitations can be identified: the semi-heuristic rule-based approach used for selection of potentially problematic census units can be further improved: the initial areal threshold for excluding units likely nonresidential was based on empirical evidence for Europe, and this threshold may not represent other geographical contexts. The success rate of this approach $(59 \%)$, although encouraging, is likely not sufficient for automating this procedure, as it yields significant false positives. Narrowing the rules to capture more extreme anomalies would decrease the rate of false positives (at the expense of increasing omission), the main priority of such data-improving exercise.

The 'split and merge' approach is conservative and suited for cases where the population in the problematic unit was enumerated but re-assigned to other neighboring units on reporting. This is a benevolent assumption on census data production that may not always hold true. Alternatives to the 'split \& merge' approach should be explored, namely contextual bottom-up approaches that take into consideration the local patterns of settlement, i.e. estimating the population in the target 'unpopulated' unit based on the average occupancy rates of adjacent census units. Despite these limitations, the approach has merits that may warrant making it publicly-available as a free tool, possibly integrated with the open and free SmartDissolve tool developed for aggregating census data in a 'smart' and flexible way (Schiavina and Freire, 2017).

Within the present population disaggregation approach, relying solely on builtup structures detected and mapped through remote sensing to inform harmonization with census is not without risks: resident population may be allocated to abandoned or seasonal settlements, or otherwise non-residential facilities (e.g. ports, industrial areas), misrepresenting the concept. Also, the proposed mitigation procedures reduces in those areas the independency in the production of the involved variables (population stats and built-up distribution), which may be desirable for subsequent combination and production of population grids and is a cornerstone of the GHSL approach.

\section{Challenges involved in creating improved global population grids using best-available census data}

The global database of censuses supporting GPW (and GHS-POP) is not yet fully open and free, with CIESIN unauthorized to re-share the census polygons for about 30 countries (including the two most populous, China and India). 
Therefore the production of the GHS-POP global grids and mitigation of detected deficiencies (Chapter 5 ) involved logistical and practical challenges to produce complete global layers: the GHS-POP grids for those 30 countries were produced by connecting remotely and running scripts in CIESIN servers in NY, USA. This reinforces the importance and need for fully open and free census data.

\subsubsection{Chapter 6: An Improved Global Analysis of Population Distribution in Proximity to Active Volcanoes, 1975-2015}

Chapter 6 sought to answer two closely related questions:

(i) How has resident population distribution and density been varying at global and regional scales (Southeast Asia, and Central America) in proximity to historically active volcanoes in the period 1975-2015, and what is the current situation?

(ii) To what extent does a new model and data on spatio-temporal distribution of population (decadal cycle) improve global and regional characterization of population living in proximity to volcanoes and the assessment of population potentially affected by their hazards?

Concerning the first question, this research shows that population is not evenly distributed across geographical space around active and historical volcanoes, and finds that overall concentrations of people in their vicinity have been increasing since 1975 . In 2015 more than 600 million people $(8.4 \%$ of world total) lived within $100 \mathrm{~km}$ of a volcano with at least one significant eruption, and more than 1 billion people (14.3\% of the total) within that distance of a Holocene volcano. The latter amount has increased by $1.57 \%$ per year since 1975, above the global population growth rate for the period. Especially significant have been the growth rates and total population living within $10 \mathrm{~km}$, which in 2015 sums 59 million for Holocene volcanoes and 21 million for those with significant eruptions, with almost half of this population living in Southeast Asia. Population densities in vicinity of volcanoes are also higher than global average densities (over land), and those densities have been increasing with time in the period analyzed. In the period under analysis, we find differences between Southeast Asia and Central America: in SE Asia the share of total population within $100 \mathrm{~km}$ of HV has increased (albeit slightly) between 1975 and 2000, and the highest growth rates occur in close proximity to HV and SV volcanoes (within $10 \mathrm{~km}$ ). In Central America the share of population within $100 \mathrm{~km}$ of HV and SV has been decreasing, and the strongest rates are observed farther away from volcanoes.

Regarding the second question, this work introduces a previously absent spatio-temporal dimension to the analysis of human settlement in proximity to historically active volcanoes, focused on contemporary times. The work exploits the potential of the first detailed, consistent, spatially-explicit time 
series of global population distribution spanning the period 1975-2015. The characteristics of the population grids developed and used (GHS-POP), namely the combination of high spatial resolution and decadal temporal scale, enable characterizing with unprecedented detail the changes in the distribution of human population in proximity to historically active volcanoes in last 40 years. This research also revises, details and updates previous analyses for current period, revealing different patterns from those previously described. The enhancements in population modeling reveal that global spatial patterns, despite being relatively stable in the temporal window considered, display a variation with distance that is not monotonic, with density generally increasing with proximity to volcanoes, peaking at around $15-20 \mathrm{~km}$ away, and then decreasing. This details and revises previous findings by Small and Naumann (2001). This work also illustrates how emerging open and free geospatial datasets can narrow gaps in population distribution data and knowledge for supporting Disaster Risk Management and Reduction activities. The revealed increase in potential human exposure may elevate disaster risk if not compensated by reductions in vulnerabilities (e.g. through preparedness, early warning).

Regarding strengths, these results are based on open and free data and a simple and transparent population distribution model. "A corollary of the transparency rule is that simple or parsimonious model representations are better than more 'sophisticated' or complex models, when they are being used for policy impact assessments" (Saltelli and Funtowicz 2014). Also, GHS-POP is the first spatially-explicit global time series relying exclusively on a proxy actually mapped (and not estimated or hind-cast) for disaggregation of population estimates in the periods considered. Expanding previous analyses, both Holocene volcanoes and those where there is evidence of significant eruptions are considered. In addition, the research has considered all volcanoes listed in the databases regardless of their type or location, as e.g., submarine volcanoes may still affect populations on land upon erupting, as many are located in proximity to inhabited areas (Lindsay et al. 2005).

In assessing population counts and densities in proximity of volcanoes, the work addressed the challenge of measuring accurately both distances and areas in global maps. The proposed approach tries to limit distortions in distances, areas, and population counts, improving over previous global analyses where this aspect is neglected (or omitted). These population grids can be combined with any type of hazard, enabling improved modeling and analyses throughout the emergency management cycle at regional and global scales.

One important limitation of such global analysis is that both the datasets of volcanoes and the population grids are affected by error and uncertainty. The analysis is carried out using a $250 \mathrm{~m}$ population grid, which makes it sensitive to uncertainty in spatial precision, although this is mitigated by the aggregation 
induced by using $2 \mathrm{~km}$ steps for distance. Uncertainties in GHS-POP mostly result from the quality and reliability of censuses and the accuracy of built-up detection in diverse landscapes using heterogeneous Landsat image archives. In volcanic rocky landscapes, commission error of built-up detection can occasionally result in misallocation of population. Such issues typically worsen with temporal distance from the current period. Despite the mitigation procedures implemented in the production of these population grids, some issues remain unsolved in global population data, such as: reporting units with unrealistically high densities, and reporting of population in uninhabited areas (i.e. over-reporting). Further, the GVP datasets (volcanoes) are affected by issues of generalization, low spatial accuracy and precision: (a) a single point frequently refers to a group of volcanoes or craters (e.g., volcanic fields) or giant calderas, for which an areal feature (polygon) would be a better representation for the potential hazard source; and (b) the geographical coordinates (decimal degrees) are often coarse with precision sometimes limited to the first decimal, which corresponds to a real world precision of about $5.5 \mathrm{~km}$. This is clearly insufficient for detailed analyses in close proximity to volcanoes and the combination of uncertainties from population and GPV discourages finer scale analyses, especially for individual volcanoes. Aware of these issues, we have tried not to convey a false sense of precision and communicate uncertainty by providing population numbers in millions (Saltelli and Funtowicz 2014).

Future avenues for research in human exposure to volcanic hazard, as pointed out in the chapter, include (i) assessing the potential threat of submarine volcanoes, and (ii) a renewed and updated look at the potential exposure of nearby cities considering their spatial characteristics, an analysis now enabled by the recent release of the GHSL-based Urban Centre Data Base (UCDB, Florczyk et al. 2019).

These population grids (GHS-POP) are being used for operational DRM (risk and impacts) in INFORM ${ }^{42}$, GDACS ${ }^{43}$, and Copernicus EMS ${ }^{44}$.

\subsection{Other research contributions: cross-disciplinary benefits and applications of the produced population distribution data for modelling, assessing impacts, and measuring access to services and resources.}

In addition to applications in the field of DRM, we have used the approaches and population models discussed in chapters 3 and 4 to advance research and knowledge in other application domains, such as:

\footnotetext{
42 https://drmkc.jrc.ec.europa.eu/inform-index

43 https://www.gdacs.org

${ }^{44}$ https://emergency.copernicus.eu
} 
- $\quad$ Planning of public facilities - access to services (Freire et al. 2011c)

- $\quad$ GeoMarketing analysis (Freire and Santos 2012)

- Environmental noise pollution analysis - exposure, impacts (Freire and Gomes 2013)

- $\quad$ Solar potential analysis (Santos et al. 2014)

At global scale, and in the domain of data provision for policy support, the GHS-POP grids provide data for monitoring of SDGs, as in analysis of Land Use Efficiency (SDG goal 11.3.1) (Melchiorri et al. 2019).

The consistent production of detailed and compatible time series of built-up and population grids has enabled developing the global modeling and classification of human settlements in space and time (i.e. the GHS-SMOD ${ }^{45}$ ) (Pesaresi and Freire, 2016). The SMOD ports in the global domain, using GHSL data, the 'degree of urbanization' method already adopted in Europe by the European Commission and EUROSTAT, and by the OECD (Dijkstra and Poelman 2014). This harmonized global definition of Cities and Settlements is peoplebased, i.e. supported by GHS-POP grids (Melchiorri et al. 2018) and has recently evolved to a more detailed classification of settlements in two levels, also called 'refined degree of urbanization' (Dijkstra et al. 2018). This definition is currently being proposed to the UN Statistical Commission for global adoption. Development of the GHS-SMOD has in turn enabled the production of the Urban Centre Database (UCDB), the most complete database on cities to date, publicly released as an open and free dataset. The UCDB represents the global status on Urban Centres in 2015 by providing information on cities location, their extent (surface, shape), and describing each city with a set of geographical, socio-economic and environmental attributes, many of them going back 25 or even 40 years in time (Florczyk et al. 2019).

Since they were first released in 2016, GHS-POP grids have so far also supported the following research: Assessment of population affected by heat waves (Ceccherini et al. 2017); visualization of global population density as interactive multi-scale map (Smith 2017); the modeling of functional urban areas (FUAs) for the whole world (Moreno-Monroy et al. 2018); quantification of Carbon footprints of 13000 cities (Moran et al. 2018); the determination of the Ranked Significance of Population Centers (Nelson and McKeon 2019); the study of the variability in urban population distributions across Africa (Tuholske et al 2019); assessment of trade-offs between urban change and food production (Wang et al. 2019); and the reporting on the demographic impact on urban growth (Gerten et al. 2019).

45 https://ghsl.jrc.ec.europa.eu/ghs_smod2019.php 


\subsection{Challenges and Way Forward}

This research introduced and addressed temporal dynamics in modeling and assessing population exposure at high spatial resolution and related applications in DRM activities. Some of the developed approaches and population grids already made their way into being adopted for operational DRM systems and activities, however addressing different needs at different scales while providing global coverage (e.g. INFORM, GDACS, Copernicus EMS Mapping). While further integration of these population grids within other risk information systems is under development (e.g. EFAS ${ }^{46}$ and GloFAS ${ }^{47}$ ), several challenges and gaps remain.

1) What is effective contribution of population distribution data to integrated DRM activities?

In this research, some insight into the value of detailed geospatial population data for DRM has been provided, showing how these data can potentially contribute to all phases. Still more research is needed into the effective contribution of population distribution data to integrated DRM activities, considering their specific requirements and the many different stakeholders (e.g. risk analysts, public and private planning authorities, emergency responders, etc.) and levels involved in DRM phases.

The research provided first-hand evidence to us that a closer coupling of researchers and DRM practitioners 'on the ground' (e.g. civil protection) is needed, both to inform the first group on the specific needs of operational DRM and to make the latter group aware of capacities and limitations of developed approaches to support those requirements. This necessitates continued efforts in further narrowing the gaps between research and development of solutions and DRM practice, to improve the 'last-mile' preparation for disasters (Taubenbock et al. 2009).

While the increased availability of global and regional population distribution grids (especially those open and free) has widened the options for users/applications, it has also fueled confusion in the user domain regarding their underlying concepts and technical specifications, and their fitness for use (e.g. Calka and Bielecka 2019). Despite recent efforts by the producers of large scale population grids at clarifying these aspects (Leyk et al. 2019), more systematic evidence-based information is needed on how population variables, concepts, and technical specifications (quality) affect and fit scientific and applied uses, namely in DRM.

2) Population dynamics of quick vs. slow-onset hazard events

\footnotetext{
46 https://www.efas.eu/

47 https://www.globalfloods.eu/
} 
This research has addressed population exposure to a set of sudden or quickonset hazard events, where it can be reasonably assumed that the baseline population grids (in respective temporal cycles) approximate the actual distribution being impacted by disaster events, especially in the absence of effective early warning systems. However, slower onset or cascading events (e.g. 2011 Great East Japan Earthquake and Tsunami) might modify this baseline representation by inducing specific population dynamics during the development of the different hazard events, requiring further research factoring human behavior (i.e. agent-based simulations) for the modeling and assessment of this evolving exposure.

\section{3) Importance of overall population counts and need to go beyond these}

This research was mostly concerned with modeling distributions of population counts and densities ${ }^{48}$. Non-withstanding the primordial importance of total population in DRM analysis and as denominator of many indicators in post2015 development agendas, additional demographic variables are also relevant for several issues and there is increasing demand for their spatiallyexplicit mapping. This calls for improved modeling of the distribution of these attributes, which can benefit from enhanced source data and modeling of total population, and also from development of specific modeling approaches and ancillary data.

4) How to detect, assess, and mitigate (in an automated way) major deficiencies present in geospatial population statistics?

This research has shown that improving (open and free) global population distribution grids requires attention to ex ante problems such as reliability and quality aspects of geospatial population statistics, such as completeness and spatial accuracy of censuses. Although these have been largely overlooked by the population modeling community, they acquired a new relevance in the frame of indicator-informed development agendas and the inclusiveness requirements of the UN pledge/commitment on 'leaving no one behind'.

Despite the detection and mitigation procedures we developed and implemented in the production of new global population grids (Freire et al. 2018a-Chapter 5), other issues remain unsolved in global population data, and more work is required to address and mitigate problems such as the existence of reporting units with unrealistically high densities, and reporting of population in uninhabited areas (i.e. over-reporting). In meantime, downstream efforts to improve modeling of population distribution should go in parallel with (and contribute to) strengthening national institutional capacities to produce better data and making it freely available to all stakeholders.

48 Although we have modeled the distribution of specific population groups (e.g. workers, students - Chapters 3 and 4). 
5) How to improve population estimates for comparable time series?

Related to the previous point, this research has created global time series of population grids spanning 40 years having unprecedented spatial detail (250 $\mathrm{m}$ ). While processing capacity and availability of detailed ancillary data (GHSL built-up areas at $30 \mathrm{~m}$ resolution) would allow modeling at higher spatial resolutions, this would be currently ill-advised in presence of significant inaccuracies and uncertainties, which in many cases would be made more conspicuous by the smaller cell size of population grids. In such a case, higher spatial resolution would be obtained while increasing uncertainty (i.e. trade-off between spatial resolution and confidence).

In addition to the inaccuracies mentioned above, the established process of producing estimates for common target years for all census units and subsequent adjustment to UN national-level estimates (Doxsey-Whitfield et al., 2015 ) is known to cause anomalous estimates, especially in small units and farther away from the census year, an issue we have signaled at an early stage of the work (Freire et al., 2016). Although this enormous harmonization task is beyond the scope of this research, improvements to this estimation and adjustment method would result in major benefits to such population grids and should be pursued. The presence of such issues discourages further increases in spatial resolution.

Assessing, representing (mapping), and communicating uncertainty remain important challenges in population distribution modeling that require more attention. In the domain of DRM, more needs to be done to meet the call for understanding, quantifying, and communicating the uncertainties and limitations of risk information, and to ensure that risk information is credible and transparent (GFDRR 2014). This research is a contribution toward improving vulnerability and exposure estimations for risk assessment.

\section{6) Top-down vs. bottom-up approaches}

Connected to the previous point, this research has employed top-down methods to disaggregate available or estimated population counts from source units to target zones. However, bottom-up methods have shown promise in some geographical contexts where population censuses are highly unreliable or outdated (Wardrop et al. 2018). However, these approaches can be timeconsuming and costly for large and heterogeneous areas, and further research is needed to optimize and improve their cost-efficiency, and on best ways to integrate with top-down approaches.

7) How to overcome classical trade-offs in modeling population distributions at high spatio-temporal resolutions?

This research has listed and discussed a number of classical trade-offs in geographical population modeling that need to be addressed in order to map their distributions at high spatio-temporal resolution with global coverage. 
Overcoming these challenges requires investing in combined improvements to input population statistics and spatial ancillary data (target zones), as we have illustrated in the ENACT project ${ }^{49}$ (Batista e Silva et al. 2018a; 2018b). Enhanced allocation of people to their estimated locations requires increasing the spatial and thematic detail of land use maps in order to better map human activities.

In disaggregating available population counts to target zones (i.e. grid cells), this research has relied on a number of ancillary data sets. At local scale and especially in data rich environments these data have been increasing in spatial, temporal, and thematic detail, with obvious benefits for population modeling, and major improvements are now reaching the continental scale (e.g. Rosina et al. 2018; Sabo et al. 2019).

Promising research paths involve combinations of conventional with unconventional data sources, including big data (from VGI, social media, mobile phones) (Aubrecht et al. 2017a; Aubrecht et al. 2018) but their use is not without problems (e.g. completeness, sustainability of approaches, data access and ownership, privacy and anonymity, representation bias) that deserve attention.

At global scale, major improvements are expected from classifying building functions and estimating their height (in urban areas), and from better detection and mapping of dispersed settlement patterns (in rural areas). However, the actual contribution of these additional descriptors of built-up areas and their cost-benefit needs investigation: data on building height is likely more relevant in urban contexts where census units are coarse and building height displays high intra-unit variation; to complicate matters, in some contexts the consideration of building height without considering its function might result in misallocation of a larger number of residents to nonresidential buildings, if these tend to be the tallest (e.g. services, office buildings, commercial). Improvements in remote sensing data (e.g. Copernicus Sentinel) and information extraction methods (e.g. machine learning) are showing promise for supplying some of these data 50 , in open and free framework (e.g. Corbane and Sabo 2019) and should be further pursued.

8) Need for more realistic representations and increased temporal resolution

This research has modeled population distributions in nighttime vs. daytime periods. Although the resident population might be an acceptable

\footnotetext{
49 https://ghsl.jrc.ec.europa.eu/enact.php

50 Sabo et al. 2019 have conducted unprecedented mapping and classification of residential vs. non-residential built-up at high spatial resolution $(10 \mathrm{~m})$ with pan-European coverage, and the layers are freely available as the European Settlement Map from Copernicus Very High Resolution data for reference year 2015, Public Release 2019.
} 
approximation to nighttime population in many contexts, more realistic representations of specific time periods are needed. Increasing accuracy in representing nighttime population involves accounting for distributions of other groups and activities, such as the modeling of visitors we have conducted in the frame of ENACT (Batista e Silva et al. 2018a), but also of those in hospitals and prisons. Although important, this is quite challenging for large, multinational areas and typically involves intensive work of identifying, mining, combining and harmonizing heterogeneous data from different sources. Especially for improving daytime representations, due consideration should be made of activities beyond work and study and their locations: people present in transportation networks, or involved in leisure and shopping activities, as well as considering tourism influx in areas and periods where that activity is significant (e.g. beachgoers for tsunami exposure). It would be as important as challenging to model people who are indoors vs. those involved in outdoor activities at useful spatial resolutions, and this would be especially valuable and relevant for exposure and risk analysis in DRM.

However, even when stocks of these population groups are successfully obtained or estimated, it has proven very challenging to derive weights (i.e. occupancy rates) for their correct disaggregation to target zones - i.e. how different is the occupancy rate of a beach in Portugal to another in Italy in a typical day of same summer month? Data from micro or specific surveys of obtained from mobile phones / LBSN may help, but their availability is still extremely limited and suffer from problems mentioned above.

Although day- vs. night is considered the most relevant temporal cycle for DRM, better representation of population dynamics requires increased temporal segmentations of population distribution, so as to represent differences on a weekly basis (workdays vs. week-end) or on a monthly or seasonal basis (winter vs. summer), as again demonstrated in frame of the ENACT project and assessment of potential population exposure (Freire et al, 2018b). Especially for response activities, the capacity for near real-time estimation of populations affected is in high demand and is likely to increase.

In conclusion: for effective support to DRM and DRR, geospatial data on population distribution should:

(i) be produced at a range of spatio-temporal scales, using best-available data,

(ii) be produced before disaster events and kept up-to-date,

(iii) be made widely available and accessible (open and free).

In our development of such data, we have tried to converge to the request from the disaster risk community for FAIR data: findable, accessible, interoperable and reusable (UNDRR 2019). 


\section{Bibliography}

Adger, W.N., 2006. Vulnerability, Global Environmental Change, 16, 268-281. Ahola T, Virrantaus K, Krisp JM and Hunter GJ, 2007. A spatio-temporal population model to support risk assessment and damage analysis for decision-making. International Journal of Geographical Information Science 21(8): 935-953.

Alçada-Almeida L, Tralhão L, Santos L, Coutinho-Rodrigues J, 2009. A multiobjective approach to locate emergency shelters and identify evacuation routes in urban areas. Geographical Analysis 41:9-29.

Alexander, D. 1996. The health effects of earthquakes in the mid-1990s, Disasters, 20, 231-247.

Alexander, D., 2006. Globalization of disaster: Trends, problems, and dilemmas, J. Int. Affairs, 59, 1-22.

Alfieri, L.; Bisselink, B.; Dottori, F.; Naumann, G.; De Roo, A.; Salamon, P.; Wyser, K.; Feyen, L.; Roo, A., 2017. Global projections of river flood risk in a warmer world. Earth's Future, 5, 171-182. doi:10.1002/2016EF000485.

Allen, T.I., Wald, D.J., Earle, P.S., Marano, K.D., Hotovec, A.J., Lin, K., Hearne, M., 2009. An Atlas of ShakeMaps and population exposure catalog for earthquake loss modeling, Bull. Earthquake Eng., vol. 7, no. 3, pp. 701718.

Ambraseys, N. and Jackson, J., 1981. Earthquake hazard and vulnerability in the northeastern Mediterranean: the Corinth earthquake sequence of February-March 1981, Disasters, 5, 355-368.

Anderson, J. R., E. E. Hardy, J. T. Roach, and R. E. Witmer. 1976. A Land Use and Land Cover Classification System for Use With Remote Sensing Data. US Geological Survey, Professional Paper No. 964.

Andredakis, I.; De Groeve, T., 2015. Towards a global humanitarian volcano impact alert model integrated into a multi-hazard system. In Detecting, Modelling and Responding to Effusive Eruptions; Harris, A.J.L., De Groeve, T., Garel, F., Carn, S.A., Eds.; Geological Society Special Publications: London, UK.

ANPC (Autoridade Nacional de Proteccao Civil), 2007. Plano Especial de Emergencia para o Risco Sısmico na Area Metropolitana de Lisboa e Concelhos Limitrofes, Lisbon, ANPC, $111 \mathrm{pp}$.

Aspinall, W.; Auker, M.; Hincks, T.; Mahony, S.; Nadim, F.; Pooley, J.; Sparks, R.S.J.; Syre, E., 2011. Volcano Hazard and Exposure in GFDRR Priority Countries and Risk Mitigation Measures-GFDRR Volcano Risk Study; Bristol University Cabot Institute and NGI Norway for the World Bank: Bristol, UK.

Aubrecht C, Kostl M, Steinnocher K, 2010. Population exposure and impact assessment: benefits of modeling urban land use in very high spatial and thematic detail. In: Tavares JMRS, Natal Jorge RM (eds) Computational Vision And Medical Image Processing, Recent Trends. Computational Methods in Applied Sciences 19. Springer, Berlin, pp 75-89.

Aubrecht C, Ozceylan D, Steinnocher K, Freire S, 2012b. Multi-level geospatial modeling of human exposure patterns and vulnerability indicators. Nat Hazards, p 17. doi:10.1007/s11069-012-0389-9. 
Aubrecht C, Ungar J, and Freire S, 2011. Exploring the potential of volunteered geographic information for modeling spatio-temporal characteristics of urban population: A case study for Lisbon Metro using Foursquare checkin data. In Proceedings of the Seventh International Conference on Virtual Cities and Territories, Lisbon, Portugal: 57-60.

Aubrecht C., D. O. Aubrecht, J. Ungar, S. Freire, K. Steinnocher, 2017a. VGDI - Advancing the concept: Volunteered Geo-Dynamic Information and its benefits for population dynamics modeling. Transactions in GIS, SI: 'Role of Volunteered Geographic Information in Advancing Science', 21(2):253276.

Aubrecht C., Freire S., Neuhold C., Curtis A. and Steinnocher K. 2012a. Introducing a temporal component in spatial vulnerability analysis, Disaster Advances, 5(2), 48-53.

Aubrecht C., Fuchs S. and Neuhold C. 2013. Spatio-temporal aspects and dimensions in integrated disaster risk management, Natural Hazards, $68(3), 1205-1216$.

Aubrecht C., J. Ungar, D. O. Aubrecht, S. Freire, K. Steinnocher, 2018. Mapping Land Use Dynamics Using the Collective Power of the Crowd. In: Mathieu PP., Aubrecht C. (eds) Earth Observation Open Science and Innovation. ISSI Scientific Report Series, vol 15, 247-253.

Aubrecht C., Steinnocher K., Köstl M., Grisel M., 2015. Dynamic population exposure modeling: Application of DynaPop-X for storm surge related coastal flood crisis management. International Geoscience and Remote Sensing Symposium 2015 (IGARSS 2015). Milano, Italy, July 2015.

Aubrecht, C., Steinnocher, K. and Huber, H., 2014. DynaPop - Population distribution dynamics as basis for social impact evaluation in crisis management. In S. R. Hiltz et al. (eds.), Proceedings of ISCRAM 2014 11 th Int. Conf. on Information Systems for Crisis Response and Management, University Park, PA, 319-323.

Aubrecht, C., Steinnocher, K., Hollaus, M., and Wagner, W., 2009. Integrating Earth observation and GIScience for high resolution spatial and functional modeling of urban land use, Computers, Environ. Urban Syst., 33, 15-25.

Aubrecht, C.; Meier, P.; Taubenböck, H., 2017b. Speeding up the clock in remote sensing: Identifying the black spots in exposure dynamics by capitalizing on the full spectrum of joint high spatial and temporal resolution. Nat. Hazards, 86, 177. doi:10.1007/s11069-015-1857-9.

Bakillah A., S Liang, A Mobasheri, J J Arsanjani \& A. Zipf, 2014. Fine-resolution population mapping using OpenStreetMap points-of interest, International Journal of Geographical Information Science, 28:9, 1940-1963, DOI: $10.1080 / 13658816.2014 .909045$

Balk, D., Deichmann, U., Yetman, G., Pozzi, F., Hay, S. I., and Nelson, A. 2006. Determining Global Population Distribution: Methods, Applications and Data, Adv Parasit, 62, 119-156, https://doi.org/10.1016/S0065308X(05)62004-0.

Balk, D., Pozzi, F., Yetman, G., Deichmann, U. and Nelson, A. 2005. The distribution of people and the dimension of place: Methodologies to improve the global estimation of urban extents, International Society for Photogrammetry and Remote Sensing Proceedings of the Urban Remote Sensing Conference, Tempe, AZ, March 2005. 
Balk, D.; Montgomery, M.R.; Engin, H.; Lin, N.; Major, E.; Jones, B., 2019. Urbanization in India: Population and Urban Classification Grids for 2011. Data, 4, 35.

Baptista MA, Heitor S, Miranda JM, Miranda P, Victor L, 1998. The 1755 Lisbon tsunami: evaluation of the tsunami parameters. J Geodyn 25(2):143-157.

Baptista MA, Miranda JM, 2009. Revision of the Portuguese catalog of tsunamis. Nat Hazards Earth Syst Sci 9:25-42.

Baptista MA, Miranda JM, Soares PM, Luis JF, 2006. Tsunami propagation along Tagus estuary (Lisbon, Portugal). Preliminary results. Sci Tsunami Hazards 24(5):329-338.

Batista e Silva F., M. Marin, K. Rosina, R. Barranco, S. Freire, and M. Schiavina, 2018a. Combining conventional and big data sources to examine spatiotemporal patterns of tourism in Europe. Tourism Management, 68:101-115.

Batista e Silva, F., K. Rosina, M. Schiavina, M. Marin, S. Freire, L. Ziemba, M. Craglia, \& C. Lavalle, 2018b. From place of residence to place of activity: towards spatiotemporal mapping of population density in Europe. Proceedings of the 21th AGILE Conference on Geographic Information Science. Lund, Sweden, June 12-15, 2018.

Bhaduri B, Bright E, Coleman P, Dobson J, 2002. LandScan: Locating People is What Matters. Geoinformatics, 5 (2):34-37.

Bhaduri, B., Bright, E., Coleman, P. and Urban, M. L., 2007. LandScan USA: a high-resolution geospatial and temporal modeling approach for population distribution and dynamics. GeoJournal 69, 103-117.

Birkmann J, 2007. Risk and vulnerability indicators at different scales: applicability, usefulness and policy implications. Environmental Hazards 7:20-31.

Birkmann, J., 2006. Measuring Vulnerability to Natural Hazards: Towards Disaster Resilient Societies, United Nations University Press.

Bowman, D. M. J. S. et al., 2017. Human exposure and sensitivity to globally extreme wildfire events. Nat. Ecol. Evol. 1, 0058.

Brown, S.K.; Auker, M.; Sparks, R., 2015. Populations around Holocene volcanoes and development of a Population Exposure Index. In Global Volcanic Hazards and Risk; Cambridge University Press: Cambridge, MA, USA, pp. 223-232.

Brown, S.K.; Jenkins, S.F.; Sparks, R.S.J.; Odbert, H.; Auker, M.R., 2017. Volcanic fatalities database: Analysis of volcanic threat with distance and victim classification. J. Appl. Volcanol, 6, 15. doi:10.1186/s13617-0170067-4.

Buchanan, M., 2001. Ubiquity - Why catastrophes happen, Three Rivers Press, New York, NY, USA.

Calka, B.; Bielecka, E., 2019. Reliability Analysis of LandScan Gridded Population Data. The Case Study of Poland. ISPRS Int. J. Geo-Inf., 8(5):222.

Cardona, O.D., M.K. van Aalst, J. Birkmann, M. Fordham, G. McGregor, R. Perez, R.S. Pulwarty, E.L.F. Schipper, and B.T. Sinh, 2012. Determinants of risk: exposure and vulnerability. In: Managing the Risks of Extreme Events and Disasters to Advance Climate Change Adaptation [Field, C.B., V. Barros, T.F. Stocker, D. Qin, D.J. Dokken, K.L. Ebi, M.D. Mastrandrea, K.J. Mach, G.-K. Plattner, S.K. Allen, M. Tignor, and P.M. Midgley (eds.)]. A Special Report of Working Groups I and II of the Intergovernmental 
Panel on Climate Change (IPCC). Cambridge University Press, Cambridge, UK, and New York, NY, USA, pp. 65-108.

Carr-Hill, R. 2013. Missing Millions and Measuring Development Progress. World Development 46: 30-44.

Carr-Hill, R. 2017. Improving Population and Poverty Estimates with Citizen Surveys: Evidence from East Africa. World Development 93: 249-259.

Carvalho, J., Cabral, J., Goncalves, R., Torres, L., and Mendes-Victor, L., 2006. Geophysical methods applied to fault characterization and earthquake potential assessment in the Lower Tagus Valley, Portugal, Tectonophysics, 418, 277-297.

CCDR-LVT, 2010. PROTAML: Diagnostico Sectorial-Riscos e Protecção Civil, Lisbon. Available at http://consulta-protaml.inescporto.pt/plano-regional. Accessed 3 January 2011.

Ceccherini G, Russo S, Ameztoy I, Marchese A F and Carmona-Moreno C, 2017. Heat waves in Africa 1981-2015, observations and reanalysis. Nat. Hazards Earth Syst. Sci. 17 115-25.

Chen K, McAneney J, Blong R, Leigh R, Hunter L, Magill C, 2004. Defining area at risk and its effect in catastrophe loss estimation: a dasymetric mapping approach. Applied Geography 24:97-111.

Chester DK, 2001. The 1755 Lisbon earthquake. Prog Phys Geogr 25(3):363383.

Chester, D.; Degg, M.; Duncan, A.; Guest, J., 2000. The increasing exposure of cities to the effects of volcanic eruptions: A global survey. Glob. Environ. Chang. Part B Environ. Hazards, 2, 89-103.

CIESIN (Center for International Earth Science Information Network). 2016. Gridded Population of the World, Version 4 (GPWv4). Palisades, NY: Columbia University. http://www.ciesin.columbia.edu/data/gpw-v4.

CIESIN (Center for International Earth Science Information Network). 2017a. Documentation for the Gridded Population of the World, Version 4 (GPWv4), Revision 10 Data Sets. Palisades, NY: NASA Socioeconomic Data and Applications Center (SEDAC), Columbia University. doi: 10.7927/H4B56GPT.

CIESIN (Center for International Earth Science Information Network). 2017b. Gridded Population of the World, Version 4 (GPWv4): Population Count, Revision 10. Palisades, NY: NASA Socioeconomic Data and Applications Center (SEDAC), Columbia University. doi:10.7927/H4PG1PPM.

Congalton, R. G., and K. Green. 2009. Assessing the Accuracy of Remotely Sensed Data: Principles and Practices. 2nd ed. Boca Raton, FL: CRC/Lewis Press.

Corbane Christina, Martino Pesaresi, Panagiotis Politis, Vasileios Syrris, Aneta J. Florczyk, Pierre Soille, Luca Maffenini, Armin Burger, Veselin Vasilev, Dario Rodriguez, Filip Sabo, Lewis Dijkstra \& Thomas Kemper, 2017. Big earth data analytics on Sentinel-1 and Landsat imagery in support to global human settlements mapping, Big Earth Data, 1:1-2, 118-144. doi: $10.1080 / 20964471.2017 .1397899$.

Corbane, C. \& Sabo, F. 2019. European Settlement Map from Copernicus Very High Resolution data for reference year 2015, Public Release 2019. European Commission, Joint Research Centre (JRC) doi: 10.2905/8BD2B792-CC33-4C11-AFD1-B8DD60B44F3B PID: http://data.europa.eu/89h/8bd2b792-cc33-4c11-afd1-b8dd60b44f3b 
Crichton D., 1999. The Risk Triangle. Natural disaster management: a presentation to commemorate the International Decade for Natural Disaster Reduction (IDNDR), 1990-2000 Ingleton J: Tudor Rose.

CUT (Coalition for Urban Transitions), 2019. Climate Emergency, Urban Opportunity. Available at: https://urbantransitions.global/en/publication/climate-emergencyurban-opportunity

Cutter S. 1996. Vulnerability to environmental hazards, Progress in Human Geography, 20(4): 529-539.

Cutter S. 2003. GI Science, Disasters and Emergency Management, Transactions in GIS, 7(4), 439-445.

Dao H. and P. Peduzzi, 2003. Global Risk and Vulnerability Index Trends per Year (GRAVITY). Technical annex and multiple risk integration Phase IV, UNDP/BCPR, Geneva.

De Bono A. and Mora M. G., 2014. A global exposure model for disaster risk assessment. International Journal of Disaster Risk Reduction, 10(B) 442451.

Deichmann, U., Ehrlich, D., Small, C., and Zeug, G., 2011. Using high resolution satellite data for identification of urban natural risk. European Union and World Bank.

Deichmann, U.; Balk, D.; Yetman, G., 2001. Transforming Population Data for Interdisciplinary Usages: From Census to Grid. CIESIN, Columbia University; Palisades, NY. Working paper available on-line at: https://sedac.ciesin.columbia.edu/gpw-v2/GPWdocumentation.pdf

Deville $P$, et al., 2014. Dynamic population mapping using mobile phone data. Proc Natl Acad Sci 111(45):15888-15893.

Dijkstra L., Florczyk A.J., Freire S., Kemper T., Pesaresi M., 2018. Applying the degree of urbanisation to the globe: a new harmonised definition reveals a different picture of global urbanization. Proceedings of the 16 th Conference of IAOS, Paris, France, 19-21 September 2018.

Dijkstra, L., Poelman, H., 2014. A harmonised definition of cities and rural areas: the new degree of urbanisation (Working Papers), Regional Working Paper 2014.

Dilley, M.; Chen, R.S.; Deichmann, U.; Lerner-Lam, A.L.; Arnold, M. 2005 Natural Disaster Hotspots: A Global Risk Analysis; The World Bank: Washington, DC, USA, 2005.

Dobson JE, 2002. War is God's Way of Teaching GIS. Proceedings of the Merrill Conference on Science at a Time of National Emergency. (http://www.merrill.ku.edu/publications/2002whitepaper/dobson.html).

Dobson JE, 2003. Estimating Populations at Risk. In: Cutter SL, Douglas BR, Wilbanks TJ (eds) Chapter 5.5 in Geographical Dimensions of Terrorism. Routledge, New York and London, pp 161-167.

Dobson, J. E., Bright, E. A., Coleman, P. R., and Bhaduri, B. L., 2003. LandScan2000: A New Global Population Geography, in: Remotely Sensed Cities, edited by: Mesev, V., Taylor \& Francis London, 267-279.

Dobson, J. E., Bright, E. A., Coleman, P. R., Durfee, R. C., and Worley, B. A., 2000. A global population database for estimating population at risk. Photogrammetric Engineering \& Remote Sensing, 66, 7, 849-857.

Dobson, J.E., 2007. In harm's way: Estimating populations at risk, in: Tools and Methods for Estimating Populations at Risk from Natural Disasters and 
Complex Humanitarian Crises, edited by: National Research Council, Washington, D.C., USA, The National Academies Press.

Douglas, J., 2007. Physical vulnerability modelling in natural hazard risk assessment, Nat. Hazards Earth Syst. Sci., 7, 283-288, doi:10.5194/nhess-7-283-2007.

Doxsey-Whitfield, E., K. MacManus, S. B. Adamo, L. Pistolesi, J. Squires, O. Borkovska, and S. R. Baptista. 2015. Taking Advantage of the Improved Availability of Census Data: A First Look at the Gridded Population of the World, Version 4. Papers in Applied Geography 1 (3): 226-234. doi:10.1080/23754931.2015.1014272.

EC (European Commission), 2010. Risk assessment and mapping guidelines for disaster management, Commission staff working paper, Brussels, 2010.

EEA (European Environment Agency), 2010. Mapping the impacts of natural hazards and technological accidents in Europe, Technical report 13/2010, Copenhagen, 2010.

EEA (European Environment Agency), 2006. The Changing Faces of Europe's Coastal Areas. Report No 6/2006, 107 p. Copenhagen: European Environment Agency.

EEA (European Environment Agency), 2016. Corine Land Cover (CLC) 2012, Version 18.5.1. https://land.copernicus. eu/pan-european/corine-landcover/clc-2012?tab=download.

Ehrlich, D.; Kemper, T.; Pesaresi, M.; Corbane, C., 2018a. Built-up area and population density: Two Essential Societal Variables to address climate hazard impact. Environ. Sci. Policy 2018, 90, 73-82. doi:10.1016/j.envsci.2018.10.001.

Ehrlich, D.; Melchiorri, M.; Florczyk, A.J.; Pesaresi, M.; Kemper, T.; Corbane, C.; Freire, S.; Schiavina, M.; Siragusa, A., 2018b. Remote Sensing Derived Built-Up Area and Population Density to Quantify Global Exposure to Five Natural Hazards over Time. Remote Sens., 10, 1378.

Eicher, C. L. and Brewer, C. A., 2001. Dasymetric Mapping and Areal Interpolation: Implementation and Evaluation, Cartogr. Geogr. Inf. S., 28, 125-138, https://doi.org/10.1559/152304001782173727

Emergency Events Database (EM-DAT), 2018. Centre for Research on the Epidemiology of Disasters (CRED). Available at https://www.emdat.be. Accessed August 2019.

Emrich, C., Cutter, S. L., and Weschler, P. J., 2011. GIS and Emergency Management, in: The SAGE Handbook of GIS and Society, edited by: Nyerges, T., Couclelis, H., and McMaster, R. B., SAGE Publications, 321343.

Esch, T., Heldens, W., Hirner, A., et al. 2017. Breaking new ground in mapping human settlements from space - the global urban footprint. ISPRS Journal of Photogrammetry and Remote Sensing, 134(December), 30-42.

Ewert, J.W.; Harpel, G.J., 2004. In harm's way: Population and volcanic risk. Geotimes, 49, 14-17.

FEMA (Federal Emergency Management Agency), 2004. Using HAZUS-MH for Risk Assessment. Technical Manual, FEMA 433. Washington, DC: Federal Emergency Management Agency. Available at www.fema.gov/HAZUS.

Ferri, S., Syrris, V., Florczyk, A., Scavazzon, M., Halkia, S. and Pesaresi M. 2014. A new map of the European settlements by automatic classification 
of 2.5-m resolution SPOT data, Proceedings of IGARSS 2014, Quebec, Canada, 13-18 July 2014.

Fisher PF, Langford M, 1996. Modeling sensitivity to accuracy in classified imagery: A study of areal interpolation by dasymetric mapping. The Professional Geographer 48:299-309.

Florczyk, A.J., Melchiorri, M., Corbane, C., Schiavina, M., Maffenini, M., Pesaresi, M., Politis, P., Sabo, S., Freire, S., Ehrlich, D., Kemper, T., Tommasi, P., Airaghi, D. and L. Zanchetta, Description of the GHS Urban Centre Database 2015, Public Release 2019, Version 1.0, Publications Office of the European Union, Luxembourg, 2019, ISBN 978-92-7999753-2, doi:10.2760/037310, JRC115586.

Foley D. L., 1952. The Daily Movement of Population into Central Business Districts. American Sociological Review, 17 (5):538-543.

Foley D. L., 1954. Urban Daytime Population: A Field for DemographicEcological Analysis. Social Forces, 32 (4):323-330.

Fraser S, Wood N, Johnston D, Leonard G, Greening P, Rossetto T, 2014. Variable population exposure and distributed travel speeds in least-cost tsunami evacuation modelling. Nat Hazards Earth Syst Sci 14:2975-2991

Freire, S., 2010. Modeling of spatio-temporal distribution of urban population at high-resolution - value for risk assessment and emergency management. In: Konecny, M., Zlatanova, S., Bandrova, T.L. (eds.), Geographic Information and Cartography for Risk and Crisis Management. Lecture Notes in Geoinformation and Cartography. Springer Berlin Heidelberg, pp. 53-67.

Freire, S., 2011. Modelação da distribuição espaço-temporal da população da Área Metropolitana de Lisboa com recurso a parâmetros empíricos, in: Trunfos de uma Geografia Activa, edited by: Santos, N. and Cunha, L., Imprensa da Universidade de Coimbra, Coimbra, Portugal, 717-723.

Freire S, Santos T, Tenedório JA, 2009. Recent urbanization and land use/land cover change in Portugal- the influence of coastline and coastal urban centers. J Coast Res SI 56:1499-1503.

Freire, S. and C. Aubrecht, 2010. Towards improved risk assessment - Mapping spatio-temporal distribution of human exposure to earthquake hazard in the Lisbon Metropolitan Area. In: Boccardo, P. et al. (Eds.): Remote Sensing and Geo-Information for Environmental Emergencies. International Symposium on Geo-information for Disaster Management (Gi4DM). CD-ROM. Torino, Italy, February 2-4, 2010.

Freire, S., T. Santos, J. A. Tenedório, 2011a. Feature extraction from satellite imagery and LiDAR to update exposure to tsunami and improve risk assessment in dynamic urban areas. In: Tavares J.M.R.S., Natal Jorge R.M. (Eds.): Computational Vision and Medical Image Processing: VipIMAGE 2011. Leiden: CRC Press/Balkema (Taylor \& Francis Group), 337-242.

Freire S, Aubrecht C, Wegscheider S, 2011b. Spatio-temporal population distribution and evacuation modeling for improving tsunami risk assessment in the Lisbon Metropolitan Area. Proceedings of International Symposium on Geo-information for Disaster Management (Gi4DM), Antalya, Turkey.

Freire, S., C. Aubrecht, A. Rodrigues, T. Santos, J. A. Tenedório, 2011c. Improving the planning of public facilities: Considering the spatiotemporal distribution of population. In N. Pinto, J.A. Tenedório, M. Santos, 
R. Deus (Eds), Proceedings of the 7th International Conference on Virtual Cities and Territories. Lisbon, October 11-13, 2001, pp. 193-196.

Freire, S. and C. Aubrecht, 2012. Integrating population dynamics into mapping human exposure to seismic hazard. Natural Hazards \& Earth Systems Sciences, 12(11), 3533-3543.

Freire, S. and T. Santos, 2012. Advancing GeoMarketing analyses with improved spatiotemporal distribution of population at high resolution. In: Nagle T. (Ed.), Proceedings of the 6th European Conference on Information Management and Evaluation (ECIME 2012). Cork, Ireland, 13-14 September, 2012, pp. 100-108.

Freire, S., C. Aubrecht, S. Wegscheider, 2012. When the Tsunami Comes to Town - Improving Evacuation Modeling by Integrating High-Resolution Population Exposure. In Rothkrantz, Ristvej, \& Franco (Eds.): ISCRAM 2012, 9th International Conference on Information Systems for Crisis Response and Management. Proceedings. Vancouver, Canada, April 2012.

Freire, S., C. Aubrecht, S. Wegscheider, 2013. Advancing tsunami risk assessment by improving spatio-temporal population exposure and evacuation modeling. Natural Hazards, 68:1311-1324.

Freire, S. and N. Gomes, 2013. Advancing environmental noise pollution analysis in urban areas by considering the variation of population exposure in space and time. Proceedings of the 29th Urban Data Management Symposium, London, UK, 29-31 May, 2013, pp. 155-160.

Freire, S. and Halkia, M., 2014. GHSL application in Europe: Towards new population grids, European Forum for Geography and Statistics, Krakow, Poland, 22-24 October 2014.

Freire S., D. Ehrlich, S. Ferri, 2015a. Population Exposure and Impacts from Earthquakes: Assessing Spatio-temporal Changes in the XX Century. Computer Modeling in Engineering \& Sciences (CMES), SI: 'Modeling of dangerous phenomena for risk mitigation'. Vol.109(2): 159-182.

Freire S., Florczyk A., Ferri S., 2015b. Modeling Day- and Nighttime Population Exposure at High Resolution: Application to Volcanic Risk Assessment in Campi Flegrei. In Palen, Büscher, Comes and Hughes (eds.), Proceedings of the 12th International ISCRAM Conference, Kristiansand, Norway, May 24-27, 2015.

Freire, S.; Florczyk, A.; Ehrlich, D. \& Pesaresi, M., 2015c. Remote Sensing Derived Continental High Resolution Built-up and Population Geoinformation for Crisis Management. Proceedings of 2015 IEEE International Geoscience \& Remote Sensing Symposium (IGARSS), 26-31 July 2015, Milan Italy, IEEE, 2015, 2677-2679.

Freire, S., T. Kemper, M. Pesaresi, A. J. Florczyk, and V. Syrris. 2015d. Combining GHSL and GPW to Improve Global Population Mapping. 2015 IEEE International Geoscience \& Remote Sensing Symposium (IGARSS), 2541-2543, Milan. doi:10.1109/IGARSS.2015.7326329.

Freire, S.; MacManus, K.; Pesaresi, M.; Doxsey-Whitfield, E.; Mills, J., 2016 a. Development of new open and free multi-temporal global population grids at $250 \mathrm{~m}$ resolution. In Proceedings of the 19th AGILE Conference on Geographic Information Science, Helsinki, Finland, 14-17 June 2016.

Freire, S.; Florczyk, A.; Pesaresi, M., 2016b. New Multi-temporal Global Population Grids-Application to Volcanism. In Proceedings of the 13th International ISCRAM Conference, Rio de Janeiro, Brazil, 22-25 May 2016. 
Freire S., M. Schiavina, A. J. Florczyk, K. MacManus, M. Pesaresi, C. Corbane, O. Borkovska, J. Mills, L. Pistolesi, J. Squires \& R. Sliuzas, 2018a. Enhanced data and methods for improving open and free global population grids: putting 'leaving no one behind' into practice. International Journal of Digital Earth. DOI: 10.1080/17538947.2018.1548656

Freire S., M. Schiavina, Batista e Silva, F., K. Rosina, 2018b. Advancing exposure and risk assessment in the EU by modeling population distribution in daily and seasonal cycles. EGU (European Geosciences Union) General Assembly 2018. Geophysical Research Abstracts, Vol. 20, EGU2018-19691-1. Vienna, Austria.

Freire, S.; Florczyk, A.J.; Pesaresi, M.; Sliuzas, R., 2019. An Improved Global Analysis of Population Distribution in Proximity to Active Volcanoes, 19752015. ISPRS Int. J. Geo-Inf. 8(8), 341.

GADM (Global Administrative Areas). 2011. Version 2.0. http://gadm.org.

Gaspar-Escribano, J. M. and Iturrioz, T., 2011. Communicating earthquake risk: mapped parameters and cartographic representation, Nat. Hazards Earth Syst. Sci., 11, 359-366, doi:10.5194/nhess-11-359-2011, 2011.

Gaughan, A. E., F. R. Stevens, C. Linard, N. N. Patel, and A. J. Tatem. 2014. Exploring Nationally and Regionally Defined Models for Large Area Population Mapping. International Journal of Digital Earth. doi:10.1080/17538947.2014.965761.

Geller, R. J., 1997. Earthquake prediction: a critical review, Geophys. J. Int., $131,425-450$.

Gerten C, Fina S and Rusche K., 2019. The Sprawling Planet: Simplifying the Measurement of Global Urbanization Trends. Front. Environ. Sci. 7:140. doi: $10.3389 /$ fenvs.2019.00140

GFDRR, 2014. Understanding risk in an evolving world - Emerging best practices in natural disaster risk assessment. Washington, DC: Global Facility for Disaster Reduction and Recovery.

Gill, J. C. and B. D. Malamud, 2014. Reviewing and visualizing the interactions of natural hazards, Rev. Geophys., 52, 680-722, doi:10.1002/2013RG000445.

Global Volcanism Program. Volcanoes of the World; Venzke, E., Ed.; Smithsonian Institution: Washington, DC, USA, 2013.

Goldewijk K and Battjes JJ, 1997. A hundred year (1890 - 1990) database for integrated environmental assessments (HYDE, version 1.1). Report no. 422514002, National Institute of Public Health and the Environment (RIVM), Bilthoven, The Netherlands.

Goodchild, M. F., 2006. GIS and Disasters: Planning for Catastrophe (Editorial), Computers, Environ. Urban Syst., 30, 227-229.

Gu, D., P. Gerland, F. Pelletier, and B. Cohen. 2015. Risks of exposure and vulnerability to natural disasters at the city level: A global overview. Population Division Technical Paper No. 2015/2. New York: United Nations Department of Economic and Social Affairs.

Guo, H., 2010. Understanding global natural disasters and the role of earth observation, Int. J. Digital Earth, 3, 221-230.

Harvey, J.T., 2002. Estimating census district populations from satellite imagery: Some

Hay, S. I., Noor, A. M., Nelson, A., \& Tatem, A. J., 2005. The accuracy of human population maps for public health application. Tropical Medicine \& 
International Health: 10(10), 1073-1086. http://doi.org/10.1111/j.13653156.2005.01487.x

Hu, L.; Wilhelmi, O.V.; Uejio, C., 2019. Assessment of heat exposure in cities: Combining the dynamics of temperature and population. Sci. Total Environ., 655, 1-12.

Huppert, H. E., \& Sparks, R. S. J. 2006. Extreme natural hazards: population growth, globalization and environmental change. Philosophical Transactions of the Royal Society A: Mathematical, Physical and Engineering Sciences, 364(1845), 1875-1888. doi:10.1098/rsta.2006.1803

INE (Instituto Nacional de Estatística), 2001. Recenseamento Geral da População e da Habitação. Lisboa.

INE (Instituto Nacional de Estatística), 2003. Movimentos Pendulares e Organização do Território Metropolitano:Área Metropolitana de Lisboa e Área Metropolitana do Porto 1991-2001. Lisbon, Portugal.

Integrated Research on Disaster Risk, 2014. Peril Classification and Hazard Glossary (IRDR DATA Publication No. 1). Beijing: Integrated Research on Disaster Risk.

IOC (Intergovernmental Oceanographic Commission), 2008. Tsunami Preparedness-Information Guide for Disaster Planners. IOC Manuals and Guides No. 49. UNESCO.

IPCC (Intergovernmental Panel on Climate Change), 2012. Managing the Risks of Extreme Events and Disasters to Advance Climate Change Adaptation. Cambridge: Cambridge University Press, 2012.

ISO, 2018. ISO 31010: Risk management - Risk assessment techniques.

Jelínek R, Krausmann E, Gonzalez M, Alvarez-Gomez J, Birkmann J, Welle T, 2012. Approaches for tsunami risk assessment and application to the city of Cádiz, Spain. Nat Hazards 60:273-293.

Johnson, G.O., 1992. GIS applications in emergency management. URISA Journal, 4(1), 66-72.

Jongman B, Ward PJ, Aerts JCJH, 2012. Global exposure to river and coastal flooding: Long term trends and changes. Global Environmental Change, 22: 823-835.

Kakhandiki, A. and Shah, H., 1998. Understanding time variation of risk: Crucial implications for megacities worldwide, Appl. Geogr., 18, 47-53.

Kellens, W., Neutens, T., Deckers, P. et al. 2012. Coastal flood risks and seasonal tourism: analysing the effects of tourism dynamics on casualty calculations. Nat Hazards 60: 1211. https://doi.org/10.1007/s11069011-9905-6.

Klupfel HL, 2005. Modelle fur die Berechnung von Personenstromen. Evakuierungssimulationen.

Lavakare, A. and Mawk, K., 2008. Exposure data quality. The Review: A guide to Catastrophe modelling. available online at: http://www.rms.com/Publications/RMS\%20Guide\%202008.pdf.

Lerner-Lam, A., 2007. Assessing global exposure to natural hazards: progress and future trends. Environmental Hazards, 7:10-19.

Lettieri E., C. Masella, G. Radaelli, 2009. Disaster management: findings from a systematic review. Disaster Prevention and Management, 18 (2).

Lewis, J.R., 2014. Usability: Lessons Learned... and Yet to Be Learned, International Journal of Human-Computer Interaction, 30:9, 663-684, DOI: $10.1080 / 10447318.2014 .930311$ 
Leyk, S., Gaughan, A. E., Adamo, S. B., de Sherbinin, A., Balk, D., Freire, S., Rose, A., Stevens, F. R., Blankespoor, B., Frye, C., Comenetz, J., Sorichetta, A., MacManus, K., Pistolesi, L., Levy, M., Tatem, A. J., and Pesaresi, M., 2019. The spatial allocation of population: a review of largescale gridded population data products and their fitness for use, Earth Syst. Sci. Data, 11, 1385-1409, https://doi.org/10.5194/essd-11-13852019.

Lima VV, Miranda JM, Baptista MA, Catalão J, Gozalez M, Otero L, Olabarrieta M, A ' Ivarez-Gómez JA, Carreño E, 2010. Impact of a 1755-like tsunami in Huelva, Spain. Nat Hazards Earth Syst Sci, 10:139-148.

Linard C. and A. Tatem, 2012. Large-scale spatial population databases in infectious disease research. International Journal of Health Geographics, $11: 7$.

Linard, C., C. W. Kabaria, M. Gilbert, A. J. Tatem, A. E. Gaughan, F. R. Stevens, A. Sorichetta, A. M. Noor, and R. W. Snow. 2017. Modelling Changing Population Distributions: An Example of the Kenyan Coast, 1979-2009. International Journal of Digital Earth, 10 (10): 1017-1029.

Lindsay, J.M., Shepherd, J.B. \& Wilson, D., 2005. Volcanic and Scientific Activity at Kick 'em Jenny Submarine Volcano 2001-2002: Implications for Volcanic Hazard in the Southern Grenadines, Lesser Antilles. Nat Hazards, 34, 1-24. https://doi.org/10.1007/s11069-004-1566-2

Lloyd C.T., H. Chamberlain, D. Kerr, G. Yetman, L. Pistolesi, F. R. Stevens, A. E. Gaughan, J. J. Nieves, G. Hornby, K. MacManus, P. Sinha, M. Bondarenko, A. Sorichetta \& A. J. Tatem, 2019. Global spatio-temporally harmonised datasets for producing high-resolution gridded population distribution datasets, Big Earth Data, 3:2, 108-139, DOI: $10.1080 / 20964471.2019 .1625151$

Lorenzoni, I.; Jordan, A.; Hulme,M.; Turner, R.K.; O’Riordan, T., 2000. A coevolutionary approach to climate change impact assessment: Part I: Integrating socio-economic and climate change scenarios. Glob. Environ. Chang., 10:57-68.

Lundgren, R. and McMakin, A., 2009. Risk Communication: A Handbook for Communicating Environmental, Safety, and Health Risks, IEEE, John Wiley \& Sons, Inc., Hoboken, NJ, USA, $4^{\text {th }}$ Edn.

Martin, D. and Bracken, I. 1991. Techniques for modelling population related raster databases, Environ. Plann. A, 23, 1069-1075, https://doi.org/10.1068/a231069.

Martin, D., Cockings, S. and Leung, S., 2010. Progress report: 24-hour gridded population models, Proceedings of European Forum for Geostatistics 2010, Tallinn, Estonia.

Martin, D., Cockings, S. and Leung, S., 2015. Developing a flexible framework for spatiotemporal population modelling. Annals of the Association of American Geographers, 105 (4), 754-772.

Martin, D., Tate, N. J., \& Langford, M., 2000. Refining population surface models: Experiments with Northern Ireland Census data. Transactions in GIS, 4(4), 343-360.

McGranahan, G., D. Balk, and B. Anderson. 2007. The Rising Tide: Assessing the Risks of Climate Change and Human Settlements in Low Elevation Coastal Zones. Environment and Urbanization, 19: 17-37.

McPherson T, Ivey A, Brown M, Streit G, 2004. Determination of the spatial and temporal distribution of population for air toxics exposure 
assessments. 5th AMS Conference on Urban Environment. Vancouver, BC, Canada.

McPherson TN, Brown MJ, 2004. Estimating daytime and nighttime population distributions in US cities for emergency response activities. In Symposium on Planning, Nowcasting and Forecasting in the Urban Zone, 84th AMS Annual Meeting, AMS, Seattle, 2004.

Melchiorri M., A. Florczyk, S. Freire, M. Schiavina, M. Pesaresi and T. Kemper, 2018. Unveiling 25 Years of Planetary Urbanization with Remote Sensing: perspectives from the Global Human Settlements Layer. Remote Sensing, 10(5): 768.

Melchiorri, M.; Pesaresi, M.; Florczyk, A.J.; Corbane, C.; Kemper, T., 2019. Principles and Applications of the Global Human Settlement Layer as Baseline for the Land Use Efficiency Indicator-SDG 11.3.1. ISPRS Int. J. Geo Inf., 2019, 8, 96.

Mennis, J. 2003. Generating Surface Models of Population Using Dasymetric Mapping, Prof. Geogr., 55, 31-42, https://www.tandfonline.com/doi/full/10.1111/0033-0124.10042

Mennis, J. and Hultgren, T. 2006. Intelligent Dasymetric Mapping and Its Application to Areal Interpolation, Cartogr. Geogr. Inf. Sc., 33, 179-194, https://doi.org/10.1559/152304006779077309

Mileti, D., 1999. Disasters by design: A reassessment of natural hazards in the United States, Washington, D.C.: Joseph Henry Press, 376 pp.

Mondal P. and A.J. Tatem, 2012. Uncertainties in Measuring Populations Potentially Impacted by Sea Level Rise and Coastal Flooding. PLoS ONE, 7(10).

Moran, D.; Kanemoto, K.; Jiborn, M.; Wood, R.; Többen, J.; Seto, K.C., 2018. Carbon footprints of 13000 cities. Environ. Res. Lett., 13, 064041.

Moreno-Monroy, A., Schiavina, M., and Veneri, P., 2018. Metropolitan areas in the world. Delineation and population trends. Journal of Urban Economics. (submitted).

Nakada, S., 2000. Hazards from pyroclastic flows and surges. In Encyclopedia of Volcanoes; Sigurdsson, H., Ed.; Academic Press: San Diego, CA, USA; pp. 945-956.

National Academies of Sciences, Engineering, and Medicine, 2017. Volcanic Eruptions and Their Repose, Unrest, Precursors, and Timing. The National Academies Press: Washington, DC, USA. doi:10.17226/24650.

Neal, D.M., 1997. Reconsidering the phases of disaster, International Journal of Mass Emergencies and Disasters, Vol. 15 No. 2, pp. 239-64.

Nelson G. D. \& R. McKeon, 2019. Peaks of People: Using Topographic Prominence as a Method for Determining the Ranked Significance of Population Centers. The Professional Geographer, 71:2, 342-354, DOI: 10.1080/00330124.2018.1531039.

Neumann B, Vafeidis AT, Zimmermann J, Nicholls RJ, 2015. Future Coastal Population Growth and Exposure to Sea-Level Rise and Coastal Flooding A Global Assessment. PLoS ONE, 10(3): e0118571. https://doi.org/10.1371/journal.pone.0118571

Nieves, J. J., Sorichetta, A., Linard, C., Bondarenko, M., Steele, J., Stevens, F., ... Tatem, A. J., 2018. Modelling Built-Settlements between RemotelySensed Observations. Preprints. doi:10.20944/preprints201812.0250.v2.

Nieves, J.J.; Stevens, F.R.; Gaughan, A.E.; Linard, C.; Sorichetta, A.; Hornby, G.; Patel, N.N.; Tatem, A.J., 2017. Examining the correlates and drivers 
of human population distributions across low- and middle-income countries. J. R. Soc. Interface, 14, 20170401. doi:10.1098/rsif.2017.0401.

Nirupama N, 2009. Analysis of the global tsunami data for vulnerability and risk assessment. Nat Hazards, 48:11-16.

NOAA (National Oceanic and Atmospheric Administration), 2019. National Geophysical Data Center World Data Service (NGDC/WDS): Significant Volcanic Eruptions Database; National Geophysical Data Center: Silver Spring, MD, USA. doi:10.7289/V5JW8BSH.

Norheim-Hagtun I and Meier $P, 2010$. Crowdsourcing for crisis mapping in Haiti. Innovations: Technology, Governance, Globalization 5: 81-9.

NRC (National Research Council), 2007. Tools and Methods for Estimating Populations at Risk from Natural Disasters and Complex Humanitarian Crises. Report by the National Academy of Sciences, National Academy Press, Washington, D.C.

NSTC (National Science and Technology Council), 2005. Tsunami risk reduction for the United States-a framework for action. NSTC, Washington.

Oliveira CS, Mota de Sá F, Ferreira MA, 2005. Application of two Different Vulnerability Methodologies to Assess Seismic Scenarios in Lisbon. Proceedings of the 250th Anniversary of the 1755 Lisbon Earthquake. November 1-4, Lisbon, Portugal.

Oliveira, C. S., 2008. Lisbon earthquake scenarios: A review on uncertainties, from earthquake source to vulnerability modeling, Soil Dynam. Earthq. Eng., 28, 890-913.

Openshaw, S. and Taylor, P. J., 1981. The modifiable areal unit problem, in: Quantitative Geography: A British View, edited by: Wrigley, N. and Bennett, R., Routledge and Kegan Paul, London.

Osaragi T., 2016. Estimation of transient occupants on weekdays and weekends for risk exposure analysis. In: 13th International Conference on Information Systems for Crisis Response and Management (ISCRAM 2016), Proceedings of the ISCRAM 2016 Conference.

Osaragi, T. and Otani, I., 2009. Estimating spatio-temporal distribution of railroad users and its application to disaster prevention planning, Lecture Notes in Geoinformation and Cartography, Advances in GIScience, Springer, 233-250.

Pan, H.; Shi, P.; Ye, T.; Xu, W.; Wang, J., 2015. Mapping the expected annual fatality risk of volcano on a global scale. Int. J. Disaster Risk Reduct., 13, 52-60.

Peduzzi P, 2004. Typology of hazards. In: Environment and poverty times no. 3. UN Environment Programme/ GRID-Arendal, Arendal, Norway, p 67.

Peduzzi, P. 2019. The Disaster Risk, Global Change, and Sustainability Nexus. Sustainability, 11, 957.

Peduzzi, P., Dao, H., Herold, C., and Mouton, F., 2009b. Assessing global exposure and vulnerability towards natural hazards: the Disaster Risk Index, Nat. Hazards Earth Syst. Sci., 9, 1149-1159, doi:10.5194/nhess9-1149-2009.

Peduzzi, P.; Chatenoux, B.; Dao, H.; De Bono, A.; Herold, C.; Kossin, J.; Mouton, F.; Nordbeck, O., 2012. Global trends in tropical cyclone risk. Nat. Clim. Chang., 2, 289-294.

Peduzzi, P.; Deichmann, U.; Maskrey, A.; Nadim, F.; Dao, H.; Chatenoux, B. ; Herold, C.; De Bono, A.; Giuliani, G., 2009a. Global disaster risk: patterns, 
trends and drivers. In Risk and Poverty in a Changing Climate; Global Assessment Report on Disaster Risk Reduction; United Nations International Strategy for Disaster Reduction: Geneva, Switzerland, pp. 17-57.

Pelling, M., 2004. Visions of risk: a review of international indicators of disaster risk and its management, UNDP Bureau for Crisis Prevention and Recovery, Geneva.

Pesaresi M. \& S. Freire, 2014. BUREF - Producing a global reference layer of built-up by integrating population and remote sensing data. GAR 2015 Background Paper for Global Risk Assessment. UNISDR, 12 p.

Pesaresi, M. \& Freire, S., 2016. GHS Settlement grid following the REGIO model 2014 in application to GHSL Landsat and CIESIN GPW v4-multitemporal (1975-1990-2000-2015). (European Commission, Joint Research Centre, 2016).

Pesaresi, M., and D. Ehrlich. 2009. A Methodology to Quantify Built-up Structures from Optical VHR Imagery. In Global Mapping of Human Settlement Experiences, Datasets, and Prospects, ch. 3, edited by P. Gamba and M. Herold, 27-58. Boca Raton, FL: CRC Press.

Pesaresi, M., D. Ehrlich, A. J. Florczyk, S. Freire, A. Julea, T. Kemper, P. Soille, and V. Syrris. 2015a. GHS Built-up Grid, Derived from Landsat, Multitemporal (1975, 1990, 2000, 2014). European Commission, Joint Research Centre (JRC) [Dataset] PID. http://data.europa.eu/89h/jrcghsl-ghs_built_Idsmt_globe_r2015b.

Pesaresi, M., D. Ehrlich, A. J. Florczyk, S. Freire, A. Julea, T. Kemper, P. Soille, and V. Syrris. 2015b. GHS Built-up Datamask Grid Derived from Landsat, Multitemporal (1975, 1990, 2000, 2014). European Commission, Joint Research Centre (JRC) [Dataset] PID. http://data.europa.eu/89h/jrcghsl-ghs_built_Idsmtdm_globe_r2015b.

Pesaresi, M., D. Ehrlich, S. Ferri, A. Florczyk, S. Freire, S. Halkia, M. J. Andreea, T. Kemper, P. Soille, and V. Syrris. 2016. Operating Procedure for the Production of the Global Human Settlement Layer from Landsat Data of the Epochs 1975, 1990, 2000, and 2014. EUR - Scientific and Technical Research Reports. Publications Office of the European Union. JRC97705. http://publications.jrc.ec.europa.eu/repository/handle/111111111/4018 2.

Pesaresi, M., G. Huadong, X. Blaes, D. Ehrlich, S. Ferri, L. Gueguen, M. Halkia, et al. 2013. A Global Human Settlement Layer from Optical HR/VHR RS Data: Concept and First Results. IEEE Journal of Selected Topics in Applied Earth Observations and Remote Sensing, 6 (5): 2102-2131. doi:10.1109/JSTARS.2013.2271445.

Pesaresi, M.; Ehrlich, D.; Ferri, S.; Florczyk, A.; Freire, S.; Haag, F.; Halkia, M.; Julea, A.M.; Kemper, T., 2015c. Soille, P. Global Human Settlement Analysis for Disaster Risk Reduction. ISPRS Int. Arch. Photogramm. Remote Sens. Spat. Inf. Sci., 40, 837-843.

Pesaresi, M.; Ehrlich, D.; Kemper, T.; Siragusa, A.; Florczyk, A.; Freire, S., 2017. Corbane, C. Atlas of the Human Planet 2017: Global Exposure to Natural Hazards; Publications Office of the European Union: Brussels, Belgium.

Pittore, M., Wieland, M. \& Fleming, K., 2017. Perspectives on global dynamic exposure modelling for geo-risk assessment. Nat Hazards, 86, 7-30. https://doi.org/10.1007/s11069-016-2437-3 
Poljanšek, K.; Marin Ferrer, M.; De Groeve, T.; Clark, I., 2017. Science for Disaster Risk Management 2017: Knowing Better and Losing Less; Publications Office of the European Union: Brussels, Belgium. doi: $10.2788 / 688605$, JRC102482.

Post J, Wegscheider S, Muck M, Zosseder K, Kiefl R, Steinmetz T, Strunz G, 2009. Assessment of human immediate response capability related to tsunami threats in Indonesia at a sub-national scale. Nat Hazards Earth Syst Sci, 9:1075-1086.

Potere D, and Schneider A, 2007. A critical look at representations of urban areas in global maps. GeoJournal, 69: 55-80.

Potere D., Schneider A., Angel S. \& Daniel L. Civco, 2009. Mapping urban areas on a global scale: which of the eight maps now available is more accurate?, International Journal of Remote Sensing, 30:24, 6531-6558, DOI: $10.1080 / 01431160903121134$

Ragona, M.; Hannstein, F.; Mazzocchi, M. 2011. The impact of volcanic ash crisis on the European Airline industry. In Governing Disasters: The Challenges of Emergency Risk regulations; Alemanno, A., Ed.; Edward Elgar Publishing: Cheltenham, UK, 2011.

Rashed, T. and Weeks, J., 2003. Assessing vulnerability to earthquake hazards through spatial multicriteria analysis of urban areas, Int. J. Geogr. Inf. Sci., 17, 547-576, 2003.

Renner, K.; Schneiderbauer, S.; Pruß, F.; Kofler, C.; Martin, D.; Cockings, S., 2018. Spatio-temporal population modelling as improved exposure information for risk assessments tested in the Autonomous Province of Bolzano. Int. J. Disaster Risk Reduct., 27, 470-479.

Rhind, D.W., 1991. Counting the people: The role of GIS. In: Maguire, D. J., Goodchild, M.F., and Rhind, D. W. (eds) Geographical Information Systems: Volume 1, Principles and Applications. London, Longman: 127137.

Riede, F., 2016. Volcanic activity and human society. Quat. Int., 394, 1-5.

Rodolfo, K.S., 2000. The hazard from lahars and Jokulhlaups. In Encyclopedia of Volcanoes; Sigurdsson, H., Ed.; Academic Press: San Diego, CA, USA; pp. 973-996.

Rogsch C, 2005. Vergleichende Untersuchungen zur dynamischen Simulation von Personenstromen. Forschungszentrum, Zentralbibliothek.

Rohat, G., 2018. Projecting drivers of human vulnerability under the shared socioeconomic pathways. International Journal of Environmental Research and Public Health, 15(3):554.

Rohat, G., Flacke, J., Dosio, A., Dao, H., \& van Maarseveen, M. 2019. Projections of human exposure to dangerous heat in African cities under multiple socioeconomic and climate scenarios. Earth's Future, 7. https://doi.org/10.1029/2018EF001020

Rosina, K., F. Batista e Silva, P. Vizcaino, M. Herrera, S. Freire \& M. Schiavina, 2018. Increasing the detail of European land use/cover data by combining heterogeneous data sets. International Journal of Digital Earth, DOI: 10.1080/17538947.2018.1550119

Sabo, F., Corbane, C., Politis, P., Pesaresi, M., \& Kemper, T., 2019. Update and improvement of the European Settlement map. 2019 Joint Urban Remote Sensing Event (JURSE), https://doi.org/10.1109/JURSE.2019.8808933 
Saltelli, A., and Funtowicz S. 2014. When All Models Are Wrong. Issues in Science and Technology 30, no. 2 (Winter 2014).

Santos T., N. Gomes, S. Freire, M.C. Brito, L. Santos, J.A. Tenedório, 2014. Applications of solar mapping in the urban environment. Applied Geography, 51: 48-57.

Satake, K. 2014. Advances in earthquake and tsunami sciences and disaster risk reduction since the 2004 Indian Ocean tsunami, Geosci. Lett., 1(15), $1-13$.

Scandone, R.; Bartolini, S.; Martí, J., 2016. A scale for ranking volcanoes by risk. Bull. Volcanol., 78, 2.

Schiavina M., S. Freire, C. Corbane, K. Rosina, \& F. Batista e Silva, 2018. Exposure to floods and earthquakes in Europe: demographic variability in daily and seasonal cycles. Proceedings of the 6th International Conference on Sustainable Development 2018. New York, USA, September 24-25, 2018.

Schiavina, M. and S. Freire, 2017. Development of a new and open approach to dissolve polygons storing count data based on areal threshold. Proceedings of the 20th AGILE Conference on Geographic Information Science. Wageningen, Netherlands, May 9-12, 2017.

Schmitt, R. C. 1956. Estimating Daytime Populations, Journal of the American Planning Association, 22: 2, $83-85$.

Semenov-Tian-Shansky, B., 1928. Russia: Territory and Population: A Perspective on the 1926 Census. Geogr. Rev., 18, 616-640, https://doi.org/10.2307/207951.

Shupeng, C., and J. van Genderen. 2008. Digital Earth in Support of Global Change Research. International Journal of Digital Earth, 1 (1): 43-65. doi:10.1080/17538940701782510.

Siebert, L.; Cottrell, E.; Venzke, E., 2015. Andrews, B. Earth's volcanoes and Their Eruptions: An Overview. In Encyclopedia of Volcanoes; Sigurdsson, H., Houghton, B., Rymer, H., Stix, J., Eds.; Academic Press: Cambridge, MA, USA.

Siebert, L.; Simkin, T.; Kimberley, P., 2010. Volcanoes of the World, 3rd ed.; University of California Press: Berkeley, CA, USA.

Small, C. and Naumann, T., 2001. The global distribution of human population and recent volcanism. Environ. Hazards, 3, 93-109.

Smith, A.; Martin, D.; Cockings, S., 2016. Spatio-temporal population modelling for enhanced assessment of urban exposure to flood risk. Appl. Spat. Anal., 9(2):145-163. doi: 10.1007/s12061-014-9110-6

Smith, D.A., 2017. Visualising world population density as an interactive multiscale map using the global human settlement population layer. Journal of Maps, 13:1, 117-123, DOI: 10.1080/17445647.2017.1400476

Smith A, Bates PD, Wing O, Sampson C, Quinn N, Neal J, 2019. New estimates of flood exposure in developing countries using high-resolution population data. Nat Commun., 10(1):1814.

Stevens, F. R., Gaughan, A. E., Linard, C., Tatem, A. J., 2015. Disaggregating census data for population mapping using Random forests with remotelysensed and ancillary data. PloS One, 10(2).

Strader, S.M.; Ashley, W.; Walker, J., 2015. Changes in volcanic hazard exposure in the Northwest USA from 1940 to 2100. Nat. Hazards, 77, 1365-1392. doi:10.1007/s11069-015-1658-1. 
Strunz G, Post J, Zosseder K, Wegscheider S, Muck M, Riedlinger T, Mehl H, Dech S, Birkmann J, Gebert N, Harjono H, Anwar HZ, Sumaryono Khomarudin RM, Muhari A, 2011. Tsunami risk assessment in Indonesia. Nat Hazards Earth Syst Sci, 11:67-82.

Sutton, P., Roberts, D., Elvidge, C., Baugh, K., 2001. Census from Heaven: an estimate of the global human population using night-time satellite imagery. International Journal of Remote Sensing, 22(16): 3061-3076.

Sutton, P.C., Elvidge, C. \& Obremski, T., 2003. Building and Evaluating Models to Estimate Ambient Population Density. Photogrammetric Engineering and Remote Sensing, 69(5), 545-553.

Tatem, A. J., A. M. Noor, C. von Hagen, A. Di Gregorio, and S. I. Hay. 2007. High Resolution Population Maps for Low Income Nations: Combining Land Cover and Census in East Africa. PLoS ONE, 2 (12): e1298.

Taubenböck H, Goseberg N, Setiadi N, Lammel G, Moder F, Oczipka M, Klupfel $H$, Wahl R, Schlurmann T, Strunz G, Birkmann J, Nagel K, Siegert F, Lehmann F, Dech S, Gress A, Klein R, 2009. "Last-Mile" preparation for a potential disaster - Interdisciplinary approach towards tsunami early warning and an evacuation information system for the coastal city of Padang, Indonesia. Nat. Hazards Earth Syst. Sci., 9:1509-1528.

Taubenböck, H., Post, J., Roth, A., Zosseder, K., Strunz, G., and Dech, S., 2008. A conceptual vulnerability and risk framework as outline to identify capabilities of remote sensing, Nat. Hazards Earth Syst. Sci., 8, 409-420.

Tenerelli, P., J. Gallego, and D. Ehrlich. 2015. Population Density Modelling in Support of Disaster Risk Assessment. International Journal of Disaster Risk Reduction, 13: 334-341. doi:10.1016/j.ijdrr.2015.07.015.

Thywissen K., 2006. Components of Risk. A Comparative Glossary, UNU Institute for Environment and Human Security.

Tieke, T., X. Liu, A. Zhang, A. Gros, et al. 2017. Mapping the world population one building at a time. Available at https://arxiv.org/abs/1712.05839

Tobin G.A., L.M. Whiteford, E.C. Jones, A.D. Murphy, S.J. Garren, C.V. Padros, 2011. The role of individual well-being in risk perception and evacuation for chronic vs. acute natural hazards in Mexico. Appl. Geogr., 31:700-711.

Tobler WR, 1979. Smooth Pycnophylactic Interpolation for Geographical Regions. Journal of the American Statistical Association, Vol. 74, No. 367.

Tobler WR, Deichmann U, Gottsegen J, Maloy K, 1995. The Global Demography Project. (Technical Report No. 95-6). National Center for Geographic Information and Analysis. UCSB. Santa Barbara, CA, 75 pp.

Tobler, W., U. Deichmann, J. Gottsegen, and K. Maloy. 1997. World Population in a Grid of Spherical Quadrilaterals. International Journal of Population Geography, 3: 203-225.

Tomlinson et al. 2011. Including the urban heat island in spatial heat health risk assessment strategies: a case study for Birmingham, UK. International Journal of Health Geographics, 10:42.

Tuholske et al., 2019. Variability in urban population distributions across Africa. Environ. Res. Lett., 14085009.

Uitto, J., 1998. The geography of disaster vulnerability in megacities, Appl. Geogr., 18, 7-16.

UNDESA (United Nations Department of Economic and Social Affairs). 2015. Department of Economic and Social Affairs, Population Division. World Population Prospects: The 2015 Revision. New York: United Nations. http://esa.un.org/unpd/wpp/DVD/. 
UNDESA (United Nations Department of Economic and Social Affairs). 2016. Sustainable Development Goals. Department of Economic and Social Affairs. Accessed March 2018. https://sustainabledevelopment.un.org/sdgs.

UNDESA (United Nations, Department of Economic and Social Affairs), 2009. Handbook on Geospatial Infrastructure in Support of Census Activities. New York, United Nations, Department of Economic and Social Affairs, Statistics Division, Series F 103.

UNDP (United Nations Development Programme), 2004. Reducing disaster risk: a challenge for development. In: Pelling M, Maskrev A, Ruiz P, Hall L (eds) United Nations Development Programme. Bureau for Crisis Prevention and Recovery, New York.

UNDP (United Nations Development Programme). 2018. Strengthening the National Statistical System. Accessed June 2018. http://www.md.undp.org/content/moldova/en/home/projects/strengthe ning-the-national-statistical-system-.html.

UNDRR (United Nations Office for Disaster Risk Reduction), 2019. Global Assessment Report on Disaster Risk Reduction, Geneva, Switzerland, United Nations Office for Disaster Risk Reduction (UNDRR).

UNECOSOC (United Nations Economic and Social Council), 2016. Report of the Inter-agency and Expert Group on Sustainable Development Goal Indicators. UN Economic and Social Council Statistical Commission 47th Session, E/CN.3/2016/2/Rev.1.

UNISDR (United Nations Office for Disaster Risk Reduction), 2009. Risk and Poverty in a Changing Climate (GAR 2009); Global Assessment Report on Disaster Risk Reduction; United Nations International Strategy for Disaster Reduction: Geneva, Switzerland, 2009.

UNISDR (United Nations Office for Disaster Risk Reduction), 2013. Global Assessment Report on Disaster Risk Reduction 2013-From Shared Risk to Shared Value: The Business Case for Disaster Risk Reduction; United Nations Office for Disaster Risk Reduction: Geneva, Switzerland.

UNISDR (United Nations Office for Disaster Risk Reduction), 2015a. Sendai framework for disaster risk reduction 2015-2030. Geneva, Switzerland: United Nations Office for Disaster Risk Reduction. http://www.unisdr.org/files/43291_sendaiframeworkfordrren.pdf

UNISDR (United Nations Office for Disaster Risk Reduction), 2015b. Making Development Sustainable: The Future of Disaster Risk Management. Global Assessment Report on Disaster Risk Reduction; United Nations Office for Disaster Risk Reduction: Geneva, Switzerland.

UNISDR (United Nations Office for Disaster Risk Reduction), 2017. Terminology on Disaster Risk Reduction. Available at https://www.unisdr.org/we/inform/terminology

United Nations International Strategy for Disaster Reduction (UNISDR): Terminology on Disaster Risk Reduction, Geneva, Switzerland, May 2009.

USGS (United States Geological Survey): The Severity of an Earthquake, USGS General Interest Publication, 2009, available at: http://pubs.usgs.gov/gip/earthq4/severitygip.html (last modified 29 April 2009, last access: 7 May 2012).

Van Westen, C. J., Damen, M. C. J., \& Feringa, W. F., 2013. Training package on national scale multi - hazard risk assessment: theory book : e-book. Kiev: PPRD-EAST. 
Van Westen, C.J., 2013. Remote sensing and GIS for natural hazards assessment and disaster risk management. In: Shroder, J. (Editor in Chief), Bishop, M.P. (Ed.), Treatise on Geomorphology. Academic Press, San Diego, CA, vol. 3, Remote Sensing and GIScience in Geomorphology, pp. 259-298.

Villagrán, J. C., 2006. Vulnerability: a conceptual and methodological review, UNU Institute for Environment and Human Security, Source Nr. 4.

Wang Y., J. van Vliet, L. Pu, P.H. Verburg, 2019. Modeling different urban change trajectories and their trade-offs with food production in Jiangsu Province, China. Computers, Environment and Urban Systems, 77 (101355).

Wardrop, N. A., W. C. Jochem, T. J. Bird, H. R. Chamberlain, D. Clarke, D. Kerr, L. Bengtsson, S. Juran, V. Seaman, and A. J. Tatem, 2018. Spatially Disaggregated Population Estimates in the Absence of National Population and Housing Census Data. Proceedings of the National Academy of Sciences, March. https://doi.org/10.1073/pnas.1715305115.

Wegscheider S, Post J, Zosseder K, Mu“ck M, Strunz G, Riedlinger T, Muhari A, Anwar $\mathrm{HZ}, 2011$. Generating tsunami risk knowledge at community level as a base for planning and implementation of risk reduction strategies. Nat Hazards Earth Syst Sci, 11:249-258.

Wieland, M.; Pittore, M.; Parolai, S.; Zschau, J., 2012. Exposure Estimation from Multi-Resolution Optical Satellite Imagery for Seismic Risk Assessment. ISPRS Int. J. Geo-Inf., 1, 69-88.

Wolff, H., H. Chong, and M. Auffhammer. 2011. Classification, Detection and Consequences of Data Error: Evidence from the Human Development Index. Economic Journal, 121 (553): 843-870.

Wood N, 2007. Variations in city exposure and sensitivity to tsunami hazards in Oregon: Reston, Va., U.S. Geological Survey Scientific Investigations Report 2007-5283.

World Bank, 2018. Improving Statistical Accuracy in Mongolia for Evidencebased Policy Making. Accessed June 2018. http://www.worldbank.org/en/results/2017/05/12/improving-statisticalaccuracy-in-mongolia-for-evidence-basedpolicy-making.

Wright, J. K., 1936. A Method of Mapping Densities of Population: With Cape Cod as an Example, Geogr. Rev., 26, 103-110.

Wu SS, Qiu X, Wang L., 2005. Population estimation methods in GIS and remote sensing: a review. GIScience and Remote Sensing, 42:80-96.

Yang, X.; Ye, T.; Zhao, N.; Chen, Q.; Yue, W.; Qi, J.; Zeng, B.; Jia, P. 2019. Population Mapping with Multisensor Remote Sensing Images and PointOf-Interest Data. Remote Sens., 11, 574.

Ye, T.; Zhao, N.; Yang, X.; Ouyang, Z.; Liu, X.; Chen, Q.; Hu, K.; Yue, W.; Qi, J.; Li, Z.; et al., 2019. Improved population mapping for China using remotely sensed and points-of-interest data within a random forests model. Sci. Total Environ., 2019, 658, 936-946.

Yuan H., X. Gao, W. Qi, 2019. Modeling the fine-scale spatiotemporal pattern of earthquake casualties in cities: Application to Haidian District, Beijing, International Journal of Disaster Risk Reduction, Vol 34:412-422. https://doi.org/10.1016/j.ijdrr.2018.12.010.

Zerger, A., and Smith D.I., 2003. Impediments to using GIS for real-time disaster decision support, Computers, Environment and Urban Systems, 27, 123-141. 
Zhang G. \& A-X. Zhu, 2018. The representativeness and spatial bias of volunteered geographic information: a review, Annals of GIS, 24:3, 151162, DOI:10.1080/19475683.2018.1501607 


\section{Summary}

Despite its importance for Disaster Risk Management (DRM), the mapping of human distribution and population exposure has lagged behind hazard modeling and mapping, in terms of accuracy, detail, and currency. As human life is the most important element to protect from disasters, assessing population exposure to actual or potential disasters can benefit all phases of the disaster management cycle, e.g. risk and impact assessment, mitigation, preparedness (including early warning and evacuation), and response. This assessment requires geo-information on population distribution at a range of spatial and temporal scales, as disasters can strike at any time and with little warning, and affect from local to global areas. However, there are significant challenges and trade-offs affecting spatio-temporal population modeling. For effective support to DRM, geospatial population data should be reliable, up-todate, have sufficient resolution (spatial, temporal, thematic), and be readily available (i.e. either produced beforehand or be rapidly computable ondemand). Such population data are still lacking for many countries and regions, either rich or poor, and conducting DRM at global scale would benefit from complete, consistent, and integrated population datasets. Furthermore, if developed adequately, such population datasets are multipurpose and can serve a wide range of application domains, such as spatial planning (urban, regional, infrastructure, public facilities), environmental assessment, epidemiology, and GeoMarketing.

This thesis comprises contributions of population distribution modeling to advancing Disaster Risk Management and Reduction efforts by: (i) developing geospatial models that improve population distribution datasets at a range of relevant spatial and temporal scales and resolutions; (ii) applying those data to (real) disaster risk scenarios by combining geospatial population layers with geophysical hazard maps; (iii) using spatial analysis for quantitatively and qualitatively assessing human exposure to specific hazards and levels, for cartographic representations and visualization, and for showing contributions to DRM. In the core chapters of the thesis, new methodologies are developed and applied to solve problems related to modeling and mapping population distribution and exposure to different hazards, aiming to improve DRM activities. Key research questions include (i) How can more detailed modeling of spatio-temporal distribution of urban population contribute to DRM, considering its cycle? (Chapter 2); (ii) Can new geospatial data on spatiotemporal distribution of population, derived by scaling-up a costly local-based approach, be combined with an earthquake hazard map to improve human exposure mapping and assessment in a metropolitan area? (Chapter 3); (iii) How is the spatio-temporal distribution of population in the daily cycle in a metropolitan area relevant for population exposure to and evacuation from tsunami hazard? (Chapter 4); (iv) How can shortcomings in geospatial 
population statistics be detected, assessed, and mitigated with remote sensing information and automated approaches, while preserving the statistic's integrity for policy making? (Chapter 5); and (v) How has resident population distribution and density been varying at global and regional scales (South East Asia, and Central America) in proximity to historically active volcanoes in period 1975-2015, and what is the current situation? (Chapter 6).

While the focus is on the spatio-temporal dynamics of human exposure, the research considers related aspects such as population definition, geospatial data and technology, spatio-temporal scales, hazard types and their characteristics, and the specific population related information requirements throughout the Disaster Risk Management Cycle. We conclude that since impacts of hazards and disasters are place and time dependent, several DRM and Disaster Risk Reduction phases and activities would benefit from relying on more spatially-detailed and time-specific assessments of population exposure, at a range of relevant spatio-temporal scales (local to global). Also, improving population distribution data for human exposure assessment requires addressing challenges present in input data and geospatial modeling. While at local scale in data rich environments more detailed and sophisticated models can be developed with acceptable uncertainty, scaling up such approaches to the global domain requires addressing different challenges, such as limitations in data availability, quality, and concepts in order to maximize the range of uses of population data, especially for supporting ongoing international development agreements. Finally, geospatial information on population distribution constitutes crucial baseline data for risk analysis and DRM across a range of hazards and threats, and investing in improving data benefits population-related analyses by detailing and revealing a sharper picture, with the aim of 'leaving no one behind'. 


\section{Samenvatting}

Hoewel belangrijk voor rampenrisicobeheersing is het in kaart brengen van de bevolkingsverdeling en -blootstelling achter gebleven bij het modelleren en in kaart brengen van risico's, in termen van nauwkeurigheid, detail en up-to-date zijn. Omdat mensenlevens het meest belangrijk zijn bij het beschermen tegen rampen, is het beoordelen van de blootstelling van de bevolking aan actuele en potentiële rampen nuttig voor alle fasen in de cyclus van rampenbeheersing, namelijk de fasen van achtereenvolgens de beoordeling van risico's en gevolgen, mitigatie, paraatheid (met inbegrip van vroegtijdige waarschuwing en evacuatie), en reactie. Deze beoordeling vereist geo-informatie van de bevolkingsverdeling in een reeks ruimte- en tijdschalen, gegeven dat rampen kunnen toeslaan op ieder moment en zonder enige waarschuwing, en hun invloed kunnen doen gelden variërend van lokale tot globale gebieden. Er zijn evenwel significante uitdagingen en trade-offs met betrekking tot het modelleren van de bevolking in ruimte en tijd. Voor een effectieve ondersteuning van rampenrisicobeheersing dienen geo-ruimtelijke bevolkingsgegevens betrouwbaar en up-to-date te zijn, een toereikende resolutie te hebben (ruimtelijk, temporeel, thematisch), en direct beschikbaar te zijn (d.w.z. van tevoren geproduceerd of snel te berekenen op aanvraag). Dergelijke bevolkingsgegevens ontbreken nog altijd in veel landen en regio's, en het uitvoeren van rampenrisicobeheersing wereldwijd zou baat hebben bij complete, consistente en geïntegreerde bevolkingsdatasets. Bovendien kunnen dergelijke bevolkingsdatasets, mits op adequate wijze gegenereerd, meerdere doeleinden dienen en gebruikt kunnen worden op meerdere terreinen, zoals ruimtelijke planning (stedelijk, regionaal, infrastructureel, openbare faciliteiten), milieu-evaluaties, epidemiologie en geo-marketing.

Deze dissertatie bevat bijdragen betreffende de modellering van de bevolkingsverdeling met als oogmerk de beheersing en reductie van rampenrisico's te bevorderen door: (i) het ontwikkelen van geo-ruimtelijke modellen die bevolkingsdatasets verbeteren voor een reeks van ruimtelijke en temporele schalen en resoluties; (ii) het toepassen van deze data op (realistische) rampenrisico scenario's door het combineren van geo-ruimtelijke layers van de bevolkingsverdeling met geofysische risicokaarten; (iii) het gebruiken van ruimtelijke analyses voor het kwantitatief en kwalitatief beoordelen van menselijke blootstelling aan specifieke gevaren en niveaus, voor het cartografisch representeren en visualiseren, en voor het illustreren van bijdragen aan rampenrisicobeheersing. In de belangrijkste hoofdstukken van deze dissertatie worden nieuwe methodologieën ontwikkeld en toegepast om problemen op te lossen die gerelateerd zijn aan het modelleren en in kaart brengen van de bevolkingsverdeling en de blootstelling aan verschillende gevaren, met als oogmerk het verbeteren van activiteiten in het kader van rampenrisicobeheersing. De belangrijkste onderzoeksvragen omvatten (i) Hoe 
kan meer gedetailleerde modellering van de ruimtelijk-temporele verdeling van de stedelijke bevolking bijdragen aan de cyclus van rampenrisicobeheersing? (Hoofdstuk 2); (ii) Kunnen nieuwe geo-ruimtelijke data over de ruimtelijktemporele verdeling van de bevolking, verkregen via opschaling van een kostbare lokale benadering, worden gecombineerd met een aardbevingsrisicokaart om het in kaart brengen en beoordelen van de menselijke blootstelling te verbeteren in een metropolitaan gebied? (Hoofdstuk 3); (iii) Hoe is de ruimtelijk-temporele bevolkingsverdeling in de dagelijkse cyclus in een metropolitaan gebied relevant voor de blootstelling en de evacuatie van de bevolking ingeval van een tsunami? (Hoofdstuk 4); (iv) Hoe kunnen tekortkomingen in geo-ruimtelijke bevolkingsstatistieken worden gedetecteerd, beoordeeld, en beperkt via remote sensing informatie en geautomatiseerde benaderingen, met behoud van de statistische integriteit voor beleidsvorming? (Hoofdstuk 5); en (v) Hoe is de variatie in bevolkingsverdeling en -dichtheid geweest op globale en regionale schaal (Zuidoost Azië, en Centraal Amerika) in de nabijheid van historisch actieve vulkanen in de periode 1975-2015, en wat is de huidige situatie? (Hoofdstuk $6)$.

Hoewel de focus van dit onderzoek ligt bij de ruimtelijk-temporele dynamiek van menselijke blootstelling, beschouwt het ook gerelateerde aspecten als de definitie van bevolking, geo-ruimtelijke data en technologie, ruimtelijktemporele schalen, gevaar typen en bijbehorende karakteristieken, als ook de specifieke vereisten die moeten worden gesteld aan bevolkingsinformatie in de volledige cyclus van rampenrisicobeheersing. Omdat de gevolgen van gevaren en rampen plaats- en tijdsafhankelijk zijn, concluderen we dat meerdere fasen van de beheersing en reductie van rampenrisico's baat hebben bij het kunnen vertrouwen op een meer ruimtelijk gedetailleerde en tijd specifieke beoordeling van de blootstelling van bevolking, over een reeks van relevante ruimtelijktemporele schalen (lokaal tot globaal). Daarnaast vereist het verbeteren van bevolkingsverdeling data voor de beoordeling van menselijke blootstelling, dat uitdagingen worden aangepakt die aanwezig zijn in invoer data en georuimtelijke modellering. Terwijl op lokale schaal in data rijke omgevingen meer gedetailleerde en geavanceerde modellen kunnen worden ontwikkeld met acceptabele nauwkeurigheid, vereist het opschalen van zulke benaderingen naar het globale domein andere uitdagingen, zoals beperkingen in data beschikbaarheid, kwaliteit, en concepten om de reikwijdte van het gebruik van bevolkingsdata te maximaliseren, in het bijzonder ter ondersteuning van lopende internationale ontwikkelingsovereenkomsten. Tenslotte bevat georuimtelijke informatie over de bevolkingsverdeling cruciale baseline data voor risicoanalyse en rampenrisicobeheersing voor een scala aan gevaren en bedreigingen, en het investeren in het verbeteren van de data is van belang voor bevolkings-gerelateerde analyses door verdergaande detaillering en het creëren van een scherper beeld, met als ultiem doel om 'niemand achter te laten'. 